\title{
CRACK-PATH FIELD AND STRAIN-INJECTION TECHNIQUES IN COMPUTATIONAL MODELING OF PROPAGATING MATERIAL FAILURE
}

\author{
J. Oliver ${ }^{1,2}$, I.F. Dias ${ }^{3}$, and A.E. Huespe ${ }^{2,4}$ \\ ${ }^{1}$ E.T.S. d'Enginyers de Camins, Canals i Ports, Technical University of Catalonia (BarcelonaTech) \\ 2International Center for Numerical Methods in Engineering (CIMNE) \\ Campus Nord UPC, Edifici C-1, c/Jordi Girona 1-3, 08034 Barcelona, Spain. \\ E-mail: xavier.oliver@upc.edu \\ ${ }^{3}$ Laboratório Nacional de Engenharia Civil (LNEC) \\ Avenida Brasil 101, 1700 Lisboa, Portugal \\ E-mail: idias@lnec.pt \\ ${ }^{4}$ INTEC-UNL-CONICET, \\ Güemes 3450, Santa Fe, Argentina. \\ E-mail: ahuespe@,intec.unl.edu.ar
}

\begin{abstract}
The work presents two new numerical techniques devised for modeling propagating material failure, i.e. cracks in fracture mechanics or slip-lines in soil mechanics. The first one is termed crack-path-field technique and is conceived for the identification of the path of those cracks, or slip-lines, represented by strain-localization based solutions of the material failure problem. The second one is termed strain-injection, and consists of a procedure to insert, during specific stages of the simulation and in selected areas of the domain of analysis, goal oriented specific strain fields via mixed finite element formulations. In the approach, a first injection, of elemental constant strain modes (CSM) in quadrilaterals, is used, in combination of the crack-path-field technique, for obtaining reliable information that anticipates the position of the crack-path. Based on this information, in a subsequent stage, a discontinuous displacement mode (DDM) is efficiently injected, ensuring the required continuity of the crack-path across sides of contiguous elements. Combination of both techniques results in an efficient and robust procedure based on the staggered resolution of the crack-path-field and the mechanical failure problems. It provides the classical advantages of the "intra-elemental" methods for capturing complex propagating displacement discontinuities in coarse meshes, as E-FEM or X-FEM methods, with the non-code-invasive character of the crack-path-field technique. Numerical representative simulations of a wide range of benchmarks, in terms of the type of material and the failure problem, show the broad applicability, accuracy and robustness of the proposed methodology. The finite element code used for the simulations is open-source and available at http:/ / www.cimne.com/compdesmat/
\end{abstract}

Keywords: fracture, computational material failure, strong discontinuities, crack-path field, strain injection, finite elements with embedded discontinuities, mixed formulations 


\section{Introduction}

In the context of this work, the concept material failure refers to the process of deterioration of the mechanical behavior in solids, produced by the reduction of the strength of the constituent material in localized domains, which, from a macroscopic view, constitute a manifold one-dimension smaller than the domain of analysis. Cracks, in fracture mechanics, and shear bands (or slip-lines), in soil mechanics, are examples of names given, in different areas of mechanics, to these failure manifolds. In addition, it is accepted that, at the macroscopic observation scale, that deterioration of the mechanical strength translates into discontinuities of the displacement field across those failure manifolds. These discontinuities in the displacement field will be technically termed strong discontinuities, in contrast with discontinuities in the strain field that are termed weak discontinuities (Mosler 2005). Moreover, it will be assumed that those failure manifolds (cracks or slip-lines) evolve along time (the term evolving discontinuities has been also coined for this case) in the sense of propagation. Once they appear they remain in a stationary position, but they can grow (propagate) in the domain of interest from the borders of the failure manifold (the crack or slip-line tips). The term strain localization will be used also to indicate that scenario in which weak discontinuities appear in the form of highly intensified strains in propagating narrow bands (the localization bands).

Material failure mechanics is a subject of large interest in simulation based sciences, where the term computational material failure mechanics has been coined. This work makes some new proposals in this area, which aim at improving the performance, in front of alternative approaches, of reliability, accuracy and robustness, of computational simulations of propagating material failure.

\subsection{Motivation}

The aim of this work is the presentation of an approach, rather than its generalization. Therefore, for the sake of simplicity, the mechanical ingredients of the approach have been simplified in some aspects: 1) the kinematical description of the motion is simplified to infinitesimal strains, 2) the dimensions of the problem, are here reduced to the 2D cases, 3) dynamic effects have been neglected and 4) thermal effects have been discarded. The authors are aware that the extension of the present work to account for some of those effects, typically 3D analysis and inertial effects, will open new, relevant and specific areas of application and, therefore, they will be considered in subsequent works.

During the last decades, a large number of proposals of models for propagating material failure have been done by the computational mechanics community, whose classification can be done on different grounds. In the context of the present work, two different classification criteria are chosen: 1) the kind of constitutive model at the failure manifold and 2) the procedure through which the displacement discontinuities, inherent to material failure modeling, are captured in the context of finite element methods.

\subsubsection{Constitutive model description of the failure manifold}

The first criterion refers to the manner that the de-cohesion process at the crack or slipline interface is modeled:

- In the so called (de)cohesive (or discrete) approaches the mechanical behavior is described in terms of a traction-separation law relating, by means of a non-linear relationship, the traction vector and the vector of displacement jump across the interface. In this law, the introduction of the fracture energy, as a material property identified as the dissipation per unit of surface along a full decohesion process, plays 
a fundamental role to make these models physically meaningful (Dugdale 1960; Hillerborg, Modeer et al. 1976; Bazant and Planas 1998).

- The continuum counterpart of the previous approach is the continuum approach, where the mechanical behavior of the interface is described in terms of a standard stress-strain constitutive model, equipped with strain softening, to account for the stress release associated to failure. The difficult point here is to relate the "interface strain" intervening in the constitutive model with the physically meaningful displacement jump. The Continum Strong Discontinuity Approach (CSDA), developed by the authors, among others, in the past (Simo, Oliver et al. 1993; Oliver 1996a), provides this link by introducing the concept of regularized strong discontinuity kinematics (a regularized version of the description of the displacement jump at the interface in terms of a Dirac's delta function) which allows describing the corresponding regularized interfacial strain. In the CSDA it is shown that any continuum stressstrain constitutive model, when applied to a strain field described by a regularized strong discontinuity kinematics induces an equivalent (projected) traction-separation law at the discontinuity interface. This provides a clear link between continuum and discrete approaches (Oliver, Huespe et al. 2002) that allows using the format of implementation considered most convenient, but keeping the physical meaning of the approach. In this sense, it can be argued in favor of the continuum approach that the same constitutive model, whenever it is equipped with strain softening, can be used for both the continuous domain and the discontinuity interface and, at a given material point, for both the undamaged and the failure stages, this leading to advantages as a less invasive implementation in commercial finite element codes or an easier identification of the material parameters.

\subsubsection{Numerical approach for displacement jumps capturing}

The second classification of interest here is the one in terms of the selected numerical approach for crack/slip-line capturing. Considering the capture of the propagating jump in the displacement field as the ultimate goal of the numerical simulation, the available approaches can be split into three groups.

- The strain-localization-based methods take advantage of the trend of continuum (stressstrain) constitutive models, equipped with strain softening, to provide solutions of the mechanical problem exhibiting strain localization in strain-localization bands. These localization-bands tend to propagate along finite element bands that, under ideal conditions, encompass just one element. In this context a strain-localization band can be interpreted as the strain field stemming from a regularized strong discontinuity kinematics, where the regularization parameter, $k$, exactly coincides with the size, $h$, of the finite-element-band ${ }^{1}(h=k)$. The fact that this numerical phenomena can be observed by just introducing strain softening in a continuum constitutive model, made this approach early used and investigated (Bazant 1983). Very soon, two large flaws were found: 1) the spurious dependence of the results on the finite element size, not showing any convergence with mesh-size refinement (mesh-size dependence) and 2) spurious dependence of the propagating localization bands on the mesh-bias (mesh-bias dependence). The numerically obtained strainlocalization bands had the trend to follow structured finite-element-bands in the mesh; therefore, slight changes in the mesh structure translate into large changes in the resulting localization bands. Even worse, when strain localization does not

\footnotetext{
${ }^{1}$ This is only exactly true if the finite element mesh is structured in finite element bands and the strain localization propagates along one of them. In most cases, the localization band jumps across finite element bands encompassing more than one element.
} 
propagate along structured finite-element-bands, the localization band encompasses more than one element in its width. This leads to mobilization of spurious stresses that cannot be released along the deformation process (stress-locking effects), and to spurious extra-dissipation of the numerical results.

Mesh-size dependence can be readily overcome by introducing the concept of regularization of the softening parameter in terms of the aforementioned fracture energy as a material property (Oliver and Huespe 2004b; Oliver, Huespe et al. 2006a). Mesh-bias dependence, instead, is not so easy to overcome. Among many attempts, the most successful ones seem to be based on relaxing the kinematical strain-displacement compatibility in the elements, by imposing it in weak form, i.e. using mixed-finite element formulations. Initial encouraging results were obtained when it was observed that mixed Q1-P0 formulations, to deal with incompressibility issues in the mechanical formulation, substantially improved the strain localization results (Zienkiewicz, Pastor et al. 1995; Cervera, Chiumenti et al. 2004; Sánchez, Sonzogni et al. 2006; Sánchez, Sonzogni et al. 2008). More recently, some improved results have been obtained using fully mixed displacement-strain formulations (Cervera, Chiumenti et al. 2010a; Cervera, Chiumenti et al. 2010b). However, in a recent paper the authors (Oliver, Huespe et al. 2012) showed that mesh-bias dependence cannot be completely (and generally) removed in strain-localization based formulations, but only alleviated until a certain degree. The reason for this was identified as the inability of the strain fields obtained in strain localization approaches to match the regularized strain fields obtained from a strong-discontinuity kinematics. Apparently, strain-localization formulations exhibit some intrinsic inabilities to completely reproduce in the general case a propagating discontinuity interface ${ }^{2}$.

- In the supra-element-band methods, a regularized displacement jump is captured by a band of finite elements encompassing several elements across its bandwidth. This bandwidth is typically of order $k$ and, therefore, the element size is $h \ll k$. Here two distinctions should be made:

- In material-regularization-based approaches (non-local models, gradientregularized models, Cosserat models (Muhlhaus and Vardoulakis 1987; Pijaudier Cabot and Bazant 1987; de Borst and Mühlhaus 1992; Lordache and Willam 1998)) continuum constitutive models, are endowed with additional ingredients that introduce a material characteristic length, $\ell$, into the material model. Then, the mathematical solution of the resulting mechanical problem, though continuous in the displacements, is compatible with high concentrations of the strains (strain-localization) in propagating bands of typical width, $\ell$, which, again, plays the role of the displacement jump regularization parameter, $k$, at the interface, and, therefore, $h \ll \ell \simeq k$.

- In the more recent phase field models for fracture (Francfort and Marigo 1998; Miehe, Hofacker et al. 2010; Miehe, Welschinger et al. 2010) diffusion-like, $k$-regularized, mechanisms inserted into a potential energy functional produce similar effects. Strain localization takes place in supra-elementalbands of bandwidth $k$ and, again, $h \ll k$.

In both cases, good results can be obtained in modeling propagating material failure, and spurious mesh-size and mesh-bias dependences are clearly overcome. Especially in the phase field models case, the method shows high potential for modeling

\footnotetext{
${ }^{2}$ In that work some mismatch indicators were developed, which become zero as the strain-localization propagates along well aligned, in advance, finite element meshes, but returned non-zero values of the indicators in general cases. It was also observed that mixed formulations released the mismatch, but neither totally nor in all cases.
} 
dynamic fracture phenomena like branching. However, also in both cases, the fact that the size of the finite element mesh $h$ is smaller than the, tinny, regularization parameter (or characteristic length) $k$ implies that a huge number of elements are required in the finite element mesh, even in simple cases. This leads to large, and sometimes unaffordable, computational costs.

- In the intra-elemental methods, instead, the discontinuity interface settles inside a propagating band encompassing only one element. The consequence is that there is no restriction in the maximum size of the finite elements capturing the discontinuity, and very coarse meshes can be used in comparison with the extraelemental methods. Besides, mesh-size and mesh-bias spurious dependences are completely overcome. As for the technique for capturing the displacement jump, this is done by enriching standard finite elements with additional spatially discontinuous displacement modes. Depending on what is the support of these discontinuous displacement modes two families arise:

- In the E-FEM based method (embedded discontinuity finite element method,(Armero and Garikipati 1996; Oliver 1996a; Alfaiate 2003; Mosler and Meschke 2003)), the enrichment is element-based. The support of the discontinuous modes is one element and the additional degrees of freedom are attached to just one element.

- In X-FEM methods (extended finite element method (Moës, Sukumar et al. 2000; Belytschko, Moes et al. 2001)) the enrichment is nodal-based. The support of the enriching discontinuity modes is nodal. i.e. all the elements sharing the same node are affected by the enriching mode.

In spite of some contradictory statements in the literature the performances of EFEM and X-FEM methods, for modeling propagating material failure in quasibrittle materials, are very similar (Oliver, Huespe et al. 2006b). Some specific benefits of E-FEM methods, in front of X-FEM methods, can be obtained due to the elemental support of the enriching modes, and the consequent condensability of the additional degrees of freedom. This brings relevant savings in terms of the computational cost and a less invasive implementation in general-purpose finite element codes. However, in both methods, robustness and accuracy depend very much on the precise determination of the position of the discontinuity interface (the crack-path or the discontinuity path) which has to be precisely determined, for every element of the band capturing the discontinuity, ensuring its continuity across sides of contiguous elements. This is classically done through the so-called crack-tracking algorithms aiming at predicting the position of the numerical crack-tip of the propagating crack, quite in advance of the physical crack-tip. These tracking algorithms (also termed zero-level-set methods in the X-FEM terminology) are cumbersome to implement, have a code-invasive character and may seriously affect the robustness of the method (Oliver, Huespe et al. 2006b; Armero 2012; Contrafatto, Cuomo et al. 2012).

\subsection{Objectives of the work}

In this work, a combination of certain newly developed numerical techniques is explored to overcome some of the deficiencies of previous and alternative approaches. The well-established continuum strong discontinuity approach (CSDA), i.e. the use of continuum (stress-strain) constitutive models, endowed with strain softening, in a regularized strong discontinuity kinematic description, constitutes the mechanical setting. The actual newness of the method is the introduction of the strain injection concept, i.e. the imposition of goal-oriented strain-fields, at specific stages of the local failure process, to 
improve the performance of the resulting model in capturing propagating material failure. This is done by resorting to mixed, displacement/strain finite element formulations restricted to specific subdomains (the injection domains) in the considered body: e.g. strictly where the improving strain fields are required.

Typically, in this work constant strain modes (CSM) in quadrilateral elements are injected to improve the flexibility of the finite elements, in a strain-localization-based approach, inside an appropriate region at the front of the propagating crack/slip-line tip. The injection is oriented to extract high-quality information on the material failure propagation. This information is used, in a subsequent stage, to perform an accurate second injection of a discontinuous-displacement mode (DDM), oriented, in turn, to the goal of capturing the mechanical behavior of the crack/slip-line. The concept of using mixed formulations in restricted domains, modifies the classical issue of finite element stability, associated to mixed formulations equally applied to the entire domain. For instance, it is shown in the paper that the CSM injection is equivalent to the classical reduced-integration technique in quadrilaterals, but the injected problem is completely stable: the classical hourglass-shaped spurious modes, typical of full-domain mixed formulations, do not show up. This suggests that the standard inf-sup conditions for stability have to be re-visited in the context of partial-domain mixed formulations (see for instance (Buscaglia and Ruas 2013)).

Selection of the injection domains and the injection times (where and when?) is based on consistent mechanical criteria; typically the discontinuous bifurcation analysis that qualifies a stress/strain state as compatible with the onset of a discontinuous displacement field. In this sense, heuristic and problem-dependent parameters in the approach are almost totally eliminated, and the whole strain injection process, evolving in time and space, takes place in a smooth and robust manner.

A second specific technique, the crack-path field technique, has been developed for the goal of identifying the spatial position of an evolving crack/slip-line, which is described by a strainlocalization field. For this purpose, a secondary problem (the crack-path-field problem) is solved and a scalar field is obtained. Then, its zero level set identifies, in advance, the candidate position for the crack-path inside every element, ensuring its continuity across sides of contiguous elements. In spite of the term here used (zero level set) the proposed technique has little in common with the level-set method used in X-FEM techniques. Here, the crack path field is identified from some specific data provided by the mechanical problem: the localized spatial distribution of the strain-like internal variable in those elements, that have been injected a constant strain mode. Once the crack-path field is obtained, the injection of the discontinuous displacement mode (DDM), which requests the availability of a predicted, inter-element continuous, crack-path, can be done trivially.

In principle, the crack-path-field and the mechanical problems are coupled in both senses. However, for practical purposes it is observed that the coupling is very weak, and a staggered procedure is suggested to solve the coupled problem: at the end of the mechanical problem, the crack-path-field-determination is reduced to a double local smoothing of the strain-like internal variable values around every element. The proposed crack-path field technique favorably replaces, as a much simpler procedure, the aforementioned cumbersome and code invasive crack-propagation algorithms, improving the locality of the implementation and the robustness of the whole method.

The organization of the remaining of the paper is the following. In section 2 , the new crack-path-field technique is developed and assessed by a number of representative numerical simulations. Then, in section 3, the strain-injection concept is introduced and applied to inject the constant strain mode (CSM) in quadrilateral elements. The, partial, benefits of this injection are then assessed through some examples. In section 4, the discontinuous displacement mode (DDM) injection is detailed and the coupled mechanicalpropagation problem is solved. In section 5 a number of representative simulations, 
covering a wide range of constitutive models, application fields (like fracture mechanics and soil stability analysis), are used to validate the applicability and the possibilities provided by the proposed approach. Finally, section 6 is devoted to formulate some concluding remarks. A number of specific issues, mostly referring to the numerical implementation aspects of the proposed approach are described in the appendices of the work. Additional information on the topics of this work can be found in (Dias 2012) and (Dias, Oliver et al. 2012).

\section{The crack-path-field technique}

In this section, a new technique for identification of the spatial position of a propagating localization field is presented. First, a strongly localized field (ideally described as a Dirac's delta-function) will be considered and a numerical setting to capture its support will be developed. In a second stage, the obtained results will be extended to the most common case of a field distributed in a localization band ${ }^{3}$.

\subsection{Identification of the path of a propagating strongly localized field}

Let us consider a body $\mathcal{B}$ in $\mathbb{R}^{n_{\text {dim }}}$, where $n_{\text {dim }}$ stands for the dimensions of the problem, defined in the time interval of interest $[0, T]$ (see Figure 1). Let us also consider a scalar field $\alpha(\mathbf{x}, t)$, propagating along time $t$, defined as:

$$
\alpha(\mathbf{x}, t)=\delta_{\mathcal{S}_{t}} \gamma_{t}(\mathbf{x})
$$

where $\delta_{\mathcal{S}_{t}}(\mathbf{x})$ stands for a Dirac's delta distribution (Stakgold 1998). In this work a field with the format of equation (1) will be considered the mathematical description of a propagating strongly localized field (and also, by extension of the name, propagating strong localization); the scalar field $\gamma_{t}(\mathbf{x}) \equiv \gamma(\mathbf{x}, t): \mathcal{B} \times[0, T] \rightarrow \mathbb{R}$ will be termed its intensity and $\mathcal{S}_{t} \subset \mathcal{B}$ will denote the path (or support) of the strong localization at time $t$, with unit normal $\mathbf{n}_{\mathcal{S}}(\mathbf{x}): \mathcal{S}_{t} \rightarrow \mathbb{R}^{n_{\mathrm{dim}}}$. The path $\mathcal{S}_{t} \subset \mathcal{B}$ is considered possibly spatially discontinuous, and limited to the support of $\alpha(\mathbf{x}, t)=\delta_{\mathcal{S}_{t}} \gamma_{t}(\mathbf{x})$ i.e.: those points of the support of the Dirac's distribution, $\delta_{\mathcal{S}_{t}}$, where the intensity $\gamma(\mathbf{x}, t)$ is not null:

$$
\gamma(\mathbf{x}, t) \equiv \gamma_{t}(\mathbf{x}) \neq 0 \quad \forall \mathbf{x} \in \mathcal{S}_{t}
$$

According with the theory of distributions, (Stakgold 1998), $\alpha(\mathbf{x})$ in equation (1) fulfills

$$
\int_{\mathcal{B}} \phi(\mathbf{x}) \alpha(\mathbf{x}) d \mathcal{B}=\int_{\mathcal{B}} \phi(\mathbf{x}) \delta_{\mathcal{S}_{t}} \gamma_{t}(\mathbf{x}) d \mathcal{B}=\int_{\mathcal{S}_{t}} \phi(\mathbf{x}) \gamma_{t}(\mathbf{x}) d \mathcal{S}
$$

for all sufficiently regular functions $\phi: \mathcal{B} \rightarrow \mathbb{R}$.

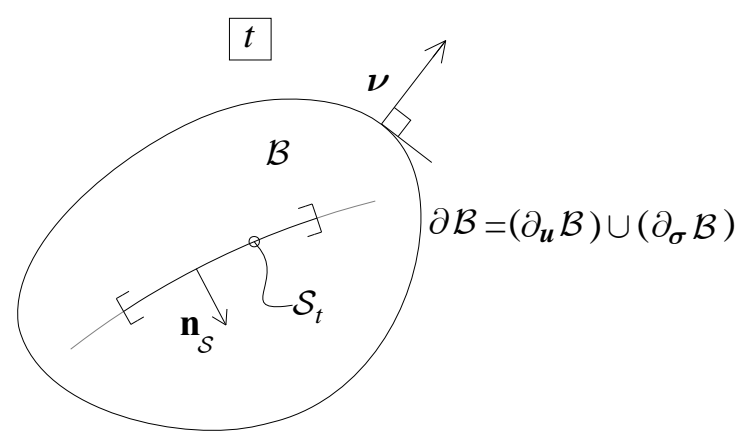

Figure 1. Propagating strong localization path

\footnotetext{
${ }^{3}$ possibly, as the result of a strain-localization based material failure modeling.
} 
Our goal here is to identify the strong-localization path $\mathcal{S}_{t} \subset \mathcal{B}$ by means of another evolving manifold, $\Gamma_{t} \subset \mathcal{B}$, associated to the solution of the following variational problem:

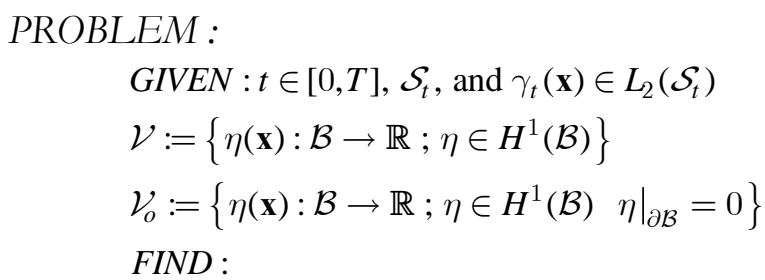
(a) $\psi(\mathbf{x}, t) \equiv \psi_{t}(\mathbf{x}) \in \mathcal{V}_{o}$ fufilling $\int_{\mathcal{B}} \bar{\psi}(\psi_{t}-\overbrace{\delta_{\mathcal{S}_{t}} \gamma_{t}}^{\alpha(\mathbf{x}, t)}) d \mathcal{B}=0 \quad \forall \bar{\psi} \in \mathcal{V}$
(b) $\mu(\mathbf{x}, t) \equiv \mu_{t}(\mathbf{x}) \in \mathcal{V}_{o}$ fufilling $\int_{\mathcal{B}} \bar{\mu}\left(\mu_{t}-\frac{\partial \psi_{t}}{\partial n}\right) d \mathcal{B}=0 \quad \forall \bar{\mu} \in \mathcal{V}$

Sub-problem (a) above identifies $\psi_{t}(\mathbf{x})$ as a smoothing of the strong-localization $\alpha(\mathbf{x}, t)=\delta_{\mathcal{S}_{t}} \gamma_{t}(\mathbf{x})$; sub-problem (b) defines $\mu_{t}(\mathbf{x})$, the crack-path-field, by smoothing the directional derivative, $\frac{\partial \psi_{t}}{\partial n} \equiv \nabla \psi_{t} \cdot \mathbf{n}_{t}(\mathbf{x})$. The field $\mathbf{n}(\mathbf{x}, t) \equiv \mathbf{n}_{t}(\mathbf{x})$ is a suitable, sufficiently smooth, extension to $\mathcal{B}$ of the normal $\mathbf{n}_{\mathcal{S}}(\mathbf{x})$ in $\mathcal{S}_{t}$, such that $\left.\mathbf{n}_{t}(\mathbf{x})\right|_{\mathbf{x}_{\mathcal{S}} \in \mathcal{S}_{t}}=\mathbf{n}_{\mathcal{S}}\left(\mathbf{x}_{\mathcal{S}}\right)$ (see Figure 1). Solution of the problem above allows defining the crack-path-set, $\Gamma_{t}$, as the zero level set of that derivative (see Figure 2 for a $1 \mathrm{D}$ sketch) i.e.:

$$
\Gamma_{t}:=\left\{\mathbf{x} \in \mathcal{B} ; \mu_{t}(\mathbf{x})=0\right\} \rightarrow \text { zero level set of } \mu_{t}(\mathbf{x})
$$

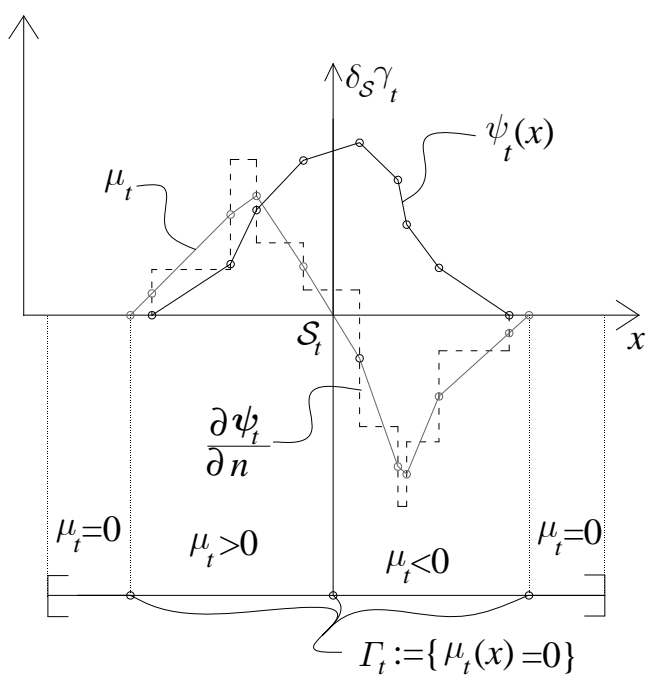

Figure 2. 1D Strong localization path-tracking problem

In view of the problem stated in equations (4) the following theorem holds:

THEOREM:

GIVEN $: \alpha(\mathbf{x}, t)=\delta_{\mathcal{S}} \gamma_{t}(\mathbf{x})$,

$\psi_{t}(\mathbf{x}), \mu_{t}(\mathbf{x})$ solutions of PROBLEM(I)

and $\Gamma_{t}:=\left\{\mathbf{x} \in \mathcal{B} ; \mu_{t}(\mathbf{x})=0\right\}$

THEN : $\mathcal{S}_{t} \subset \Gamma_{t}$ 


\section{PROOF:}

Let us consider equation (4)-(a) with the specific test function:

$$
\bar{\psi}(\mathbf{x}) \equiv \bar{\xi}(\mathbf{x}) \mu_{t}(\mathbf{x}) \in \mathcal{V} \quad \forall \bar{\xi}(\mathbf{x}) \in \mathcal{E}:=\left\{\bar{\xi} \in H^{1}(\mathcal{B}) ; \frac{\partial \bar{\xi}(\mathbf{x})}{\partial n}=0\right\}
$$

which yields

$$
\int_{\mathcal{B}} \bar{\xi} \mu_{t} \psi_{t} d \mathcal{B}=\int_{\mathcal{B}} \bar{\xi} \mu_{t} \delta_{\mathcal{S}_{t}} \gamma_{t} d \mathcal{B}=\int_{\mathcal{S}_{t}} \bar{\xi} \mu_{t}(\mathbf{x}) \gamma_{t}(\mathbf{x}) d \mathcal{S} \quad \forall \bar{\xi} \in \mathcal{E}
$$

where equation (3) has been considered. Now from equation (4)-(b), with $\bar{\mu}_{t}(\mathbf{x}) \equiv \bar{\xi}(\mathbf{x}) \psi_{t}(\mathbf{x}) \in \mathcal{V}$, we obtain:

$$
\begin{aligned}
& \int_{\mathcal{B}} \bar{\xi} \psi_{t} \mu_{t} d \mathcal{B}=\int_{\mathcal{B}} \bar{\xi} \mu_{t} \psi_{t} d \mathcal{B}=\int_{\mathcal{B}} \bar{\xi} \psi_{t} \frac{\partial \psi_{t}}{\partial n} d \mathcal{B}= \\
& =\int_{\mathcal{B}} \frac{\partial}{\partial n}\left(\frac{1}{2} \bar{\xi} \psi_{t}^{2}\right) d \mathcal{B}=\int_{\partial \mathcal{B}}(\frac{1}{2} \bar{\xi} \underbrace{\overline{\psi_{t}^{2}}}_{=0}) \boldsymbol{\nu} \cdot \mathbf{n} d \Gamma=0 \quad \forall \bar{\xi} \in \mathcal{E}
\end{aligned}
$$

where integration by parts has been applied, and the restriction $\frac{\partial \bar{\xi}}{\partial n}=0$, in equation (7), has also been considered. In equation (9) $\nu$ is the outward normal to the boundary $\partial \mathcal{B}$, and condition $\left.\psi_{t}(\mathbf{x})\right|_{\partial \mathcal{B}}=0 \forall \psi_{t} \in \mathcal{V}_{o}$ (see equation (4)) has been applied. Now combining equations (8) and (9):

$$
\begin{aligned}
& \int_{\mathcal{B}} \bar{\xi} \mu_{t} \psi_{t} d \mathcal{B}=\int_{\mathcal{S}_{t}} \bar{\xi} \mu_{t} \gamma_{t} d \mathcal{S}=0 \quad \forall \bar{\xi} \in \mathcal{E} \\
& \Rightarrow \mu_{t}(\mathbf{x}) \gamma_{t}(\mathbf{x})=0 \quad \forall \mathbf{x} \in \mathcal{S}_{t}
\end{aligned}
$$

Since, from equation (2), $\gamma_{t}(\mathbf{x}) \neq 0 \forall \mathbf{x} \in \mathcal{S}_{t}$ in view of equation (6) and the definition in equation (4)-(c) equation (10) yields:

$$
\mu_{t}(\mathbf{x})=0 \quad \forall \mathbf{x} \in \mathcal{S}_{t} \Rightarrow \mathcal{S}_{t} \subset \Gamma_{t}
$$

which proves the theorem.

REMARK 2.1-1 Equation (11) states that the strong localization path $\mathcal{S}_{t}$ lies at the interior of the manifold $\Gamma_{t}$ defined in equation (5). This is a crucial element of the crack-path-field strategy described in next sections

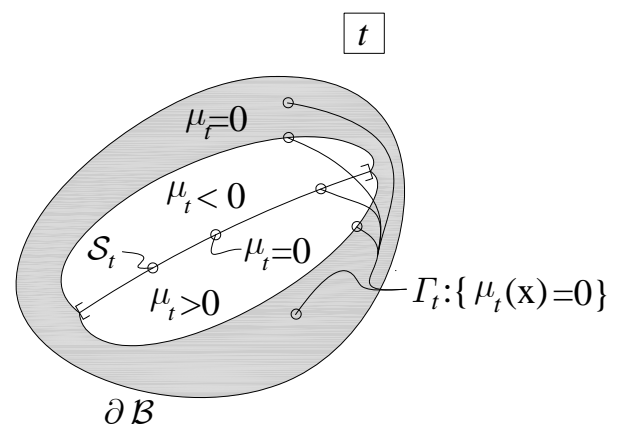

Figure 3. Typical evolving strong localization path, $\mathcal{S}_{t}$, and the corresponding crackpath-set $\Gamma_{t}$ (zero-level-set of the crack-path-field $\mu_{t}(\mathbf{x})$ )

\subsection{Tracking the path of a regularized strong localization}

Let us now consider a material failure/fracture problem in a domain. $\mathcal{B}^{h}$, discretized in a finite element mesh of typical size $h$, where a propagating crack/slip-line is aimed at being modeled using a strain localization technique. Disregard the constitutive model used to capture the strong localization (local, non-local, gradient-type etc.) let us assume that a 
scalar localized strain-like internal variable, $\alpha^{h}(\mathbf{x}, t)$ is available, which is always positive and never decreasing, i.e.:

$$
\left\{\begin{array}{l}
\alpha^{h}(\mathbf{x}, 0)=0 \\
\dot{\alpha}^{h}(\mathbf{x}, t)=0 \text { in elastic unloading } \\
\dot{\alpha}^{h}(\mathbf{x}, t)>0 \text { in inelastic loading }
\end{array}\right.
$$

We shall assume that the considered strain-localization problem is a certain regularization of a propagating strong localization field, with the format in equation (1), which arises from a strong-discontinuity problem: i.e. a problem involving jumps in the displacement field and a Dirac's delta function in the strain field.

The exact propagating path of that strong localization is unknown, and the available localized internal variable $\alpha^{h}(\mathbf{x}, t)$ in equation (12) is then considered a b-based regularization of that theoretical strongly localized field, propagating across $\mathcal{B}$. It is considered smeared in a band of finite elements, the localization domain $\mathcal{B}_{\text {loc }}^{h}(t) \subset \mathcal{B}$, evolving along time (see Figure 4) whose bandwidth, $h_{l o c}(h)$, depends on the finite element mesh size $\left(h_{l o c}=\mathcal{O}(h)\right)$.

The goal now is to determine the path, $\mathcal{S}_{t}$ (see Figure 4), of the unknown strong localization, $\alpha(\mathbf{x}, t)=\delta_{\mathcal{S}} \bar{\alpha}_{t}(\mathbf{x})$, by means of an evolving manifold, $\Gamma_{t}^{h} \subset \mathcal{B}^{h}$,

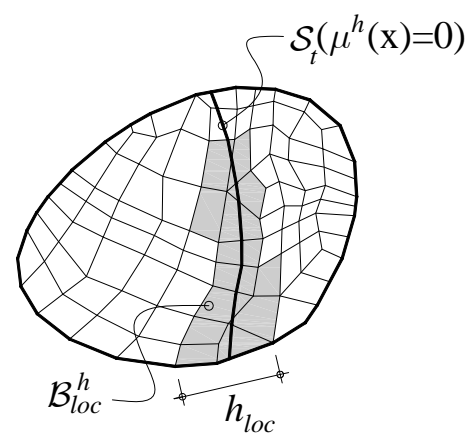

Figure 4. 2D Localization domain

whose placement is given by the discretized version of the problem in equation (4), as shown in BOX 2.2-1:

PROBLEM:

\section{BOX 2.2-1: Crack-path-field problem}

GIVEN : $\alpha^{h}(\mathbf{x}, t): \mathcal{B}^{h} \times[0, T] \rightarrow \mathbb{R}^{+}$,

$\mathbf{n}(\mathbf{x}, t): \mathcal{B}^{h} \times[0, T] \rightarrow \mathbb{I}:=\left\{\mathbf{v} \in \mathbb{R}^{3} \mid\|\mathbf{v}\|=1\right\}$

$\nu^{h}:=\left\{\eta^{h}(\mathbf{x})=\sum_{1}^{n_{\text {node }}} N_{i}(\mathbf{x}) \eta_{i} ; \eta^{h} \in H^{1}\left(\mathcal{B}^{h}\right)\right\}$

$\mathcal{V}_{0}^{h}:=\left\{\eta^{h}(\mathbf{x})=\sum_{1}^{n_{\text {node }}} N_{i}(\mathbf{x}) \eta_{i} ; \eta^{h} \in H^{1}\left(\mathcal{B}^{h}\right) ;\left.\eta^{h}\right|_{\partial \mathcal{B}^{h}}=0\right\}$

FIND :

(a) $\psi_{t}^{h}(\mathbf{x}) \in \mathcal{V}_{o}^{h}$ fufilling $\int_{\mathcal{B}^{h}} \bar{\psi}^{h}\left(\psi_{t}^{h}-\alpha^{h}(\mathbf{x}, t) d \mathcal{B}=0 \quad \forall \bar{\psi}^{h} \in \mathcal{V}^{h}\right.$

(b) $\mu_{t}^{h}(\mathbf{x}) \in \mathcal{V}_{o}^{h}$ fufilling $\int_{\mathcal{B}^{h}} \bar{\mu}^{h}(\mu_{t}^{h}-\underbrace{\frac{\partial \psi_{t}^{h}}{\partial n}}_{\nabla_{t}^{h} \cdot \mathbf{n}}) d \mathcal{B}=0 \quad \forall \bar{\mu}^{h} \in \mathcal{V}^{h}$

(c) $\Gamma_{t}^{h}:=\left\{\mathbf{x} \in \mathcal{B}^{h} ; \mu_{t}^{h}(\mathbf{x})=0\right\} \rightarrow$ zero level set of $\mu_{t}^{h}(\mathbf{x})$ 
where $N_{i}(\mathbf{x})$ are the standard shape functions and $\eta_{i}$ are the nodal values of the field $\eta^{h}(\mathbf{x})$ . The field $\mu_{t}^{h}(\mathbf{x})$ is the crack-path-field whose zero level set defines the crack-path-set, $\Gamma_{t}^{h}$, which, by means of the THEOREM in equation (6), contains the actual crack path $\mathcal{S}_{t}$ ( $\left.\mathcal{S}_{t} \subset \Gamma_{t}^{h}\right)$.

In Figure 5 the 1D version of the fields involved in BOX 2.2-1 is presented. Details on the finite element implementation of the method can be found in Appendix A.3.

(a)

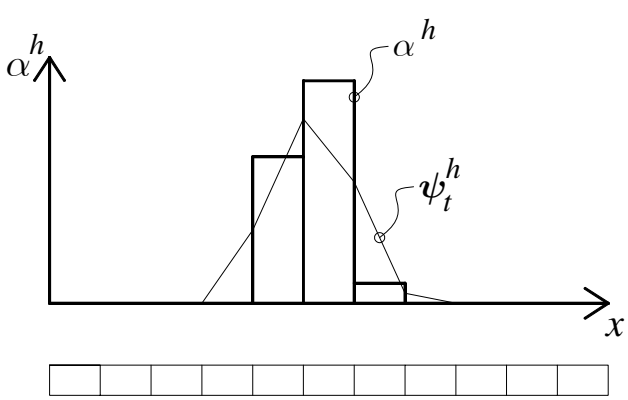

(b)

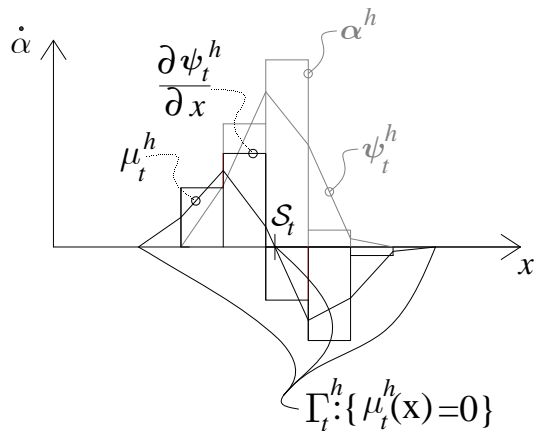

Figure 5 Finite element discretized 1D problem. (a) distribution of a localizing strainlike internal variable, $\alpha^{h}(x)$ and its smoothed counterpart $\psi_{t}^{h}(x)$, (b) distribution of the derivative $\frac{\partial \psi_{t}^{h}(x)}{\partial x}$, the crack-path-field, $\mu_{t}^{h}(x)$, and the crack path set $\Gamma_{t}^{h}$ identified as the zero level set of $\mu_{t}^{h}(x)$.

\subsection{A representative simulation}

The following example illustrates the procedure presented in previous sections. We consider the double cantilever concrete beam, reported in (Kobayashi, Hawkins et al. 1985), which is loaded as indicated in Figure 6-(a). The experiment displays an inclined straight crack propagating from the notch tip as shown in the figure. This crack, and the corresponding structural response, is aimed at being captured by using a (plane-stress) isotropic continuum damage model (Oliver, Cervera et al. 1990). The strain softening parameter, $\mathcal{H}=\ell \overline{\mathcal{H}}$, is conveniently regularized in terms of a characteristic length, $\ell(h)$ (Oliver 1989), and the intrinsic softening modulus, $\overline{\mathcal{H}}\left(G_{f}\right)$ characterized in terms of the fracture energy, $G_{f}$, in order to do the results objective with respect to the finite element size $h$.

Standard bi-linear quadrilateral finite elements are used for capturing the localization process. Figure 6-(b) shows the localization pattern in terms of the (amplified) deformed mesh, and Figure 6-(c)-(d) shows the evolution of the localization domain, $\mathcal{B}_{l o c}^{h}(t)$ at two different stages of the analysis.

(a)

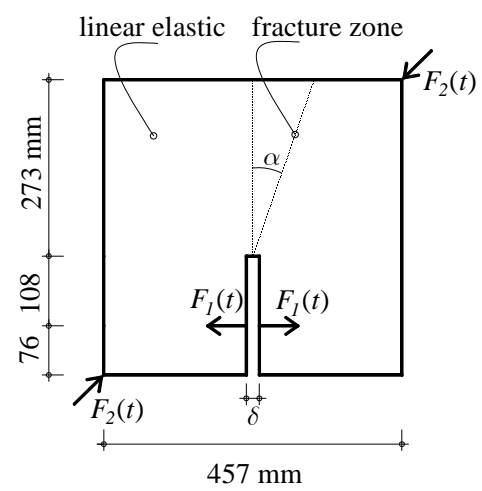

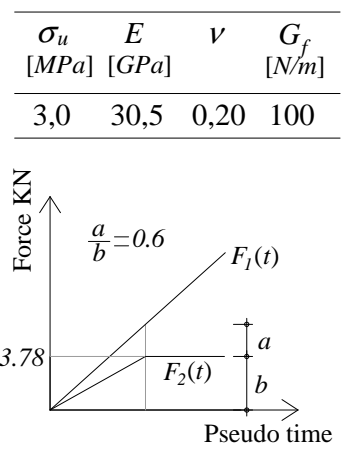

(b)

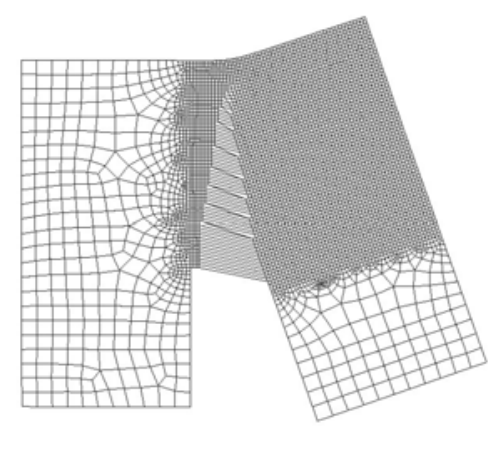


(c) $t=1$

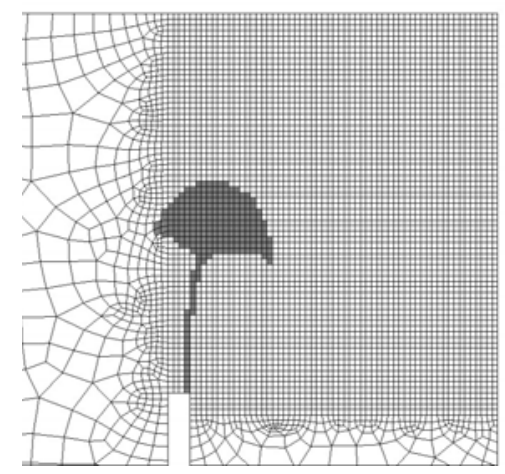

(e) $t=1$

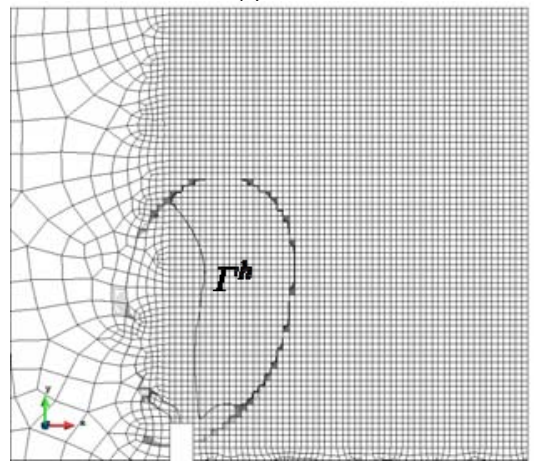

(d) $\mathrm{t}=2$

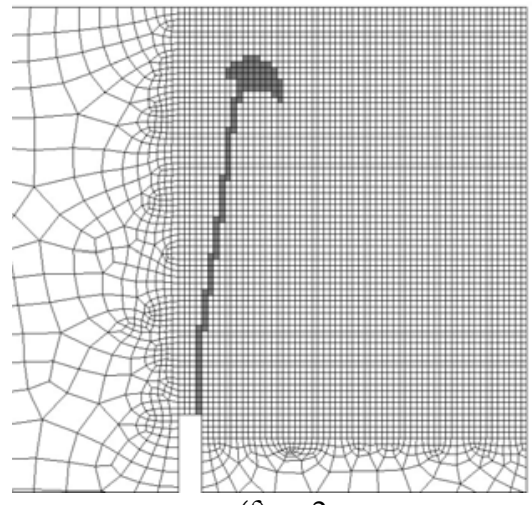

(f) $t=2$

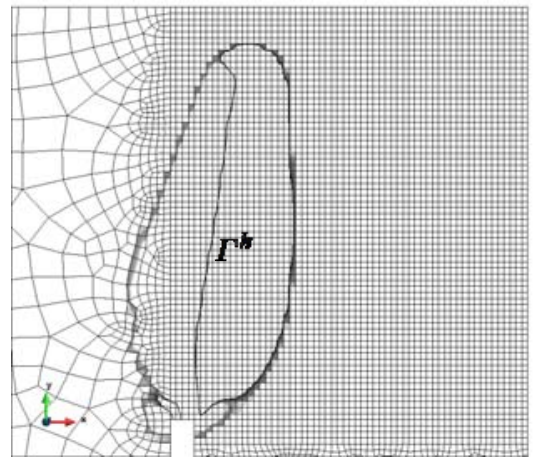

Figure 6 Double cantilever beam with diagonal loads (Kobayashi, Hawkins et al. 1985): (a) geometric, loading data and material properties of the isotropic damage model, being $\sigma_{u}$ the ultimate stress, $E$ the Young's modulus, $\nu$ the Poisson's ratio and $G_{f}$ the fracture energy, (b) deformed (amplified) mesh displaying the strain localization, (c)-(d) Localization domain, $\mathcal{B}_{l o c}^{h}(t)$, at two specific stages of the analysis and (e)-(f) computed crack path set $\Gamma^{h}$.

Figure 6-(e)-(f) displays the corresponding evolution of the crack-path-set, $\Gamma^{h}$, obtained as the solution of the problem in BOX 2.2-1. As it can be checked in the figure the central part of the set, $\mathcal{S}^{h}$, resembles the experimentally observed crack-path, with some mismatch in the orientation angle. Interestingly enough, that numerically computed crack path has been obtained with the only information of the localized strain-like internal variable field, $\alpha^{h}(\mathbf{x}, t)$, and the vector field, $\mathbf{n}(\mathbf{x})$.

REMARK 2.3-1. It is worth emphasizing that the proposed procedure does not require specific information on the geometrical position and evolution of the strain-localization domain, $\mathcal{B}_{\text {loc }}^{h}(t)$, in Figure 6-(c)-(d) ${ }^{4}$, but only knowledge of the numerical description of the localized variable $\alpha(\mathbf{x}, t)$. In this sense, it can be use in the context of any type of strain-localization formulation, for identification of the crack path associated to strainlocalization patterns (Dufour, Pijaudier-Cabot et al. 2008).

\footnotetext{
${ }^{4}$ which is computed, just for plotting purposes ,by means of methods presented in sections below.
} 


\section{The strain-injection concept}

\subsection{Variational approaches to the mechanical problem}

\subsubsection{One-field ( $u$ ) variational problem}

Let us consider the body $\mathcal{B}$ in Figure 1 with boundary $\partial \mathcal{B}=\left(\partial_{u} \mathcal{B}\right) \cup\left(\partial_{\sigma} \mathcal{B}\right)$ where $\partial_{u} \mathcal{B}$ and $\partial_{\sigma} \mathcal{B}$ are, respectively, the portions of the boundary $\partial \mathcal{B}$, where Dirichlet and Newman conditions are defined, and $\nu$ is its outward normal. Let us now consider the mechanical problem in $\mathcal{B}$, considering infinitesimal strains and the quasi-static case, stated in terms of the following boundary value problem:

PROBLEM

$$
\begin{aligned}
& \text { FIND: } \quad u(\mathbf{x}, t): \mathcal{B} \times[0, T] \rightarrow \mathbb{R}^{n_{\text {dim }}} \\
& \varepsilon(\mathbf{x}, t): \mathcal{B} \times[0, T] \rightarrow \mathbb{S}^{n_{\text {dim }} \times n_{\text {dim }}} \\
& \sigma(\mathbf{x}, t): \mathcal{B} \times[0, T] \rightarrow \mathbb{S}^{n_{\text {dim }} \times n_{\text {dim }}} \\
& \text { FULFILLING : } \\
& \boldsymbol{\nabla} \cdot \boldsymbol{\sigma}+\mathbf{b}=\mathbf{0} \rightarrow \text { equilibrium equation } \\
& \varepsilon=\boldsymbol{\nabla} \otimes^{s} \boldsymbol{u} \equiv \boldsymbol{\nabla}^{s} \boldsymbol{u} \rightarrow \text { compatibiliy equation }(b) \\
& \sigma(\mathbf{x}, t)=\Sigma(\varepsilon) \rightarrow \text { constitutive equation } \\
& \left.\begin{array}{ll}
\boldsymbol{u}=\boldsymbol{u}^{*}(\mathbf{x}, t) & \forall \mathbf{x} \in \partial_{u} \mathcal{B} \\
\boldsymbol{\sigma} \cdot \boldsymbol{\nu}=\mathbf{t}^{*}(\mathbf{x}, t) & \forall \mathbf{x} \in \partial_{\sigma} \mathcal{B}
\end{array}\right\} \rightarrow\left\{\begin{array}{c}
\text { boundary } \\
\text { conditions }
\end{array}\right.
\end{aligned}
$$

where $u, \varepsilon$ and $\sigma$ stand, respectively, for the displacements, the infinitesimal strains and the stresses, $\boldsymbol{\nabla} \otimes^{s} \boldsymbol{u} \equiv \boldsymbol{\nabla}^{s} \boldsymbol{u}$ stands for the symmetric gradient of the displacements, $\boldsymbol{u}^{*}(\mathbf{x}, t)$ , $\mathbf{t}^{*}(\mathbf{x}, t)$ and $\mathbf{b}(\mathbf{x}, t)$ are the prescribed displacements, tractions and body forces, and $\boldsymbol{\Sigma}(\bullet)$ stands for the constitutive equation supplying the stresses in terms of the strains. The onefield variational counterpart of the problem in equations (14) reads:

\section{PROBLEM}

GIVEN :

$$
\begin{aligned}
& \mathcal{V}:=\left\{\boldsymbol{\eta}_{t}(\mathbf{x}) \in H^{1}(\mathcal{B}) ;\left.\boldsymbol{\eta}_{t}(\mathbf{x})\right|_{\partial_{u} \mathcal{B}}=u^{*}(\mathbf{x}, t)\right\} \\
& \mathcal{V}_{0}:=\left\{\boldsymbol{\eta}(\mathbf{x}) \in H^{1}(\mathcal{B}) ;\left.\boldsymbol{\eta}(\mathbf{x})\right|_{\partial_{u} \mathcal{B}}=\mathbf{0}\right\}
\end{aligned}
$$

FIND :

$$
u_{t}(\mathbf{x}) \equiv \boldsymbol{u}(\mathbf{x}, t): \mathcal{B} \times[0, T] \rightarrow \mathbb{R}^{n_{\text {dim }}} ; u_{t} \in \mathcal{V}
$$

FULFILLING :

$$
\int_{\mathcal{B}} \nabla^{s} \boldsymbol{\eta}: \boldsymbol{\Sigma}\left(\boldsymbol{\nabla}^{s} \boldsymbol{u}_{t}\right) d \mathcal{B}-W^{e x t}\left(\boldsymbol{\eta}, \mathbf{b}, \mathbf{t}^{*}\right)=\mathbf{0} \quad \forall \boldsymbol{\eta} \in \mathcal{V}_{0}
$$

Equation (15) is the classical virtual work principle, where $W^{\text {ext }}\left(\boldsymbol{\eta}, \mathbf{b}, \mathbf{t}^{*}\right)$ is the standard external virtual work. As classically done in this context, the algebraic equations (14)-(b) (strain-displacement compatibility) and (14)-(c) (constitutive equation) are point-wise fulfilled, whereas the equilibrium equation (14)-(a) is imposed in weak form through the integral equation (15).

\subsubsection{Two-field $u / \varepsilon$ variational problem}

An alternative variational approach to the problem in equation (14) is the one based on two fields, displacements and strain, $\boldsymbol{u} / \boldsymbol{\varepsilon}$, as follows (for convenience in future developments, see REMARK 4.2-6, the equations are formulated in rate form and the notation $(\cdot) \equiv \frac{\partial(\cdot)(\mathbf{x}, t)}{\partial t}$ is used): 
GIVEN :

$$
\begin{aligned}
\mathcal{V} & :=\left\{\boldsymbol{\eta}_{t}(\mathbf{x}) \in H^{1}(\mathcal{B}) ;\left.\boldsymbol{\eta}_{t}(\mathbf{x})\right|_{\partial_{u} \mathcal{B}}=\dot{\boldsymbol{u}}^{*}(\mathbf{x}, t)\right\} \\
\mathcal{V}_{0} & :=\left\{\boldsymbol{\eta}(\mathbf{x}) \in H^{1}(\mathcal{B}) ;\left.\boldsymbol{\eta}(\mathbf{x})\right|_{\partial_{u} \mathcal{B}}=\mathbf{0}\right\} \\
\mathcal{E} & :=\left\{\boldsymbol{\mu}(\mathbf{x}) \in\left[L^{2}(\mathcal{B})\right]^{n_{\mathrm{dim}} \times n_{\mathrm{dim}}}\right\}
\end{aligned}
$$

FIND:

$$
\begin{array}{ll}
\dot{u}_{t}(\mathbf{x}) \equiv \dot{u}(\mathbf{x}, t): \mathcal{B} \times[0, T] \rightarrow \mathbb{R}^{n_{\mathrm{dim}}} & ; \dot{u}_{t} \in \mathcal{V} \\
\dot{\varepsilon}_{t}(\mathbf{x}) \equiv \dot{\varepsilon}(\mathbf{x}, t): \mathcal{B} \times[0, T] \rightarrow \mathbb{S}^{n_{\mathrm{dim}} \times n_{\text {dim }}} \quad ; \dot{\varepsilon}_{t} \in \mathcal{E}
\end{array}
$$

FULFILLING :

$$
\begin{aligned}
& \int_{\mathcal{B}} \nabla^{s} \boldsymbol{\eta}: \dot{\boldsymbol{\Sigma}}\left(\dot{\varepsilon}_{t}\right) d \mathcal{B}-W^{e x t}\left(\boldsymbol{\eta}, \dot{\mathbf{b}}, \dot{\mathbf{t}}^{*}\right)=0 \\
& \int_{\mathcal{B}} \boldsymbol{\mu}:\left(\dot{\varepsilon}_{t}-\nabla^{s} \dot{\boldsymbol{u}}_{t}\right) d \mathcal{B}=0
\end{aligned}
$$

Equations (17)-(a)-(b) enforce, in weak form, the equilibrium equation and kinematic compatibility equations (14)-(a)-(b), whereas the constitutive equation (14)-(c) is point-wise enforced.

\begin{abstract}
REMARK 3.1-1 The two-field format of equations (17) opens a number of additional possibilities for modeling material failure. In contrast with the one-field approach, in equation (15), the problem can be inserted in the context of the assumed strain methods (Simó and Ju 1987), this providing additional freedom in the choice of the strains. This fact will be conveniently exploited in subsequent sections.
\end{abstract}

\title{
3.2 Strain injection technique
}

By using the strain injection concept a number of strain rate fields, $\dot{\varepsilon}(\mathbf{x}, t)$, patterns can be imposed at specific domains of the body $\mathcal{B}$. In particular, we are interested in the following setting (see BOX 3.2-1):

1. A injection domain $\mathcal{B}_{i n j}(t) \subset \mathcal{B}$ is constructed as the union of $n_{i n j}$, disjoint and evolving along time, domains, $\mathcal{B}_{i n j}^{(i)}(t)$ (see Figure 7 ), each one equipped with a specific incremental (in rate form) assumed strain field, $\dot{\varepsilon}_{i n j}^{(i)}(\mathbf{x}, t)$. The remaining domain $\mathcal{B} \backslash \mathcal{B}_{i n j}(t)$ is equipped with the point wise compatible strain $\nabla^{s} \boldsymbol{u}(\mathbf{x}, t)$. In addition, the incremental strains are injected during domainspecific time intervals, []$_{i n j}^{(i)}$, not necessarily disjoint, belonging to the total interval of interest $[0, T]$ :

$$
\begin{aligned}
& \mathcal{B}_{\text {inj }}(t)=\bigcup_{i=1}^{i=n_{i n j}} \mathcal{B}_{i n j}^{(i)}(t) \\
& \mathcal{B}_{i n j}^{(i)} \subset \mathcal{B} \quad \mathcal{B}_{i n j}^{(i)} \cap \mathcal{B}_{i n j}^{(j)}=\varnothing i, j \in\left\{1,2, . . n_{i n j}\right\} \\
& \dot{\varepsilon}(\mathbf{x}, t)=\dot{\varepsilon}_{i n j}^{(i)}(\mathbf{x}, t) \quad \forall \mathbf{x} \in \mathcal{B}_{i n j}^{(i)}(t) \quad i \in\left\{1,2 \ldots, n_{i n j}\right\} \quad \forall t \in[]_{i n j}^{(i)} \subset[0, T] \\
& \dot{\varepsilon}(\mathbf{x}, t)=\dot{\varepsilon}^{(0)}(\mathbf{x}, t)=\nabla^{s} \dot{u}(\mathbf{x}, t) \quad \forall \mathbf{x} \in \mathcal{B} \backslash \mathcal{B}_{\text {inj }}(t) \quad \forall t \in[0, T]
\end{aligned}
$$

2. The injection domains, $\mathcal{B}_{i n j}^{(i)}(t)$, and the injected strains, $\dot{\varepsilon}_{i n j}^{(i)}(\mathbf{x}, t)$, are selected on goal-oriented basis. For modeling computational material failure purposes, they should be chosen to minimize some of the classical problems found in 
one-field finite element approaches, typically: mesh-bias dependence of the modeled propagating localization, and the well-known stress-locking phenomena.

Generalization to this setting of the two field $(u-\varepsilon)$ variational problem in equations(16) and (17) is presented in BOX 3.2-1.

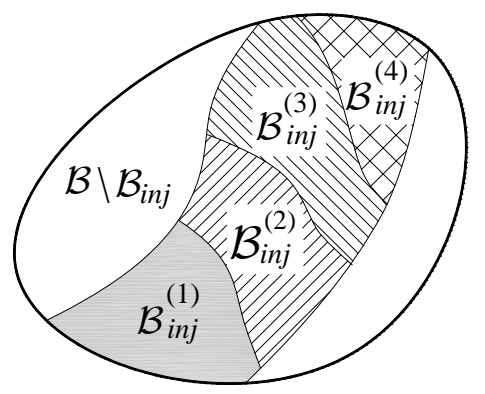

Figure 7. Strain injection: the injection domains, $\mathcal{B}_{i n j}^{(i)}(t)$, are injected an assumed (rate of) strain, $\dot{\varepsilon}_{i n j}^{(i)}(\mathbf{x}, t)$, in the specific time interval []$_{i n j}^{(i)} \subset[0, T]$

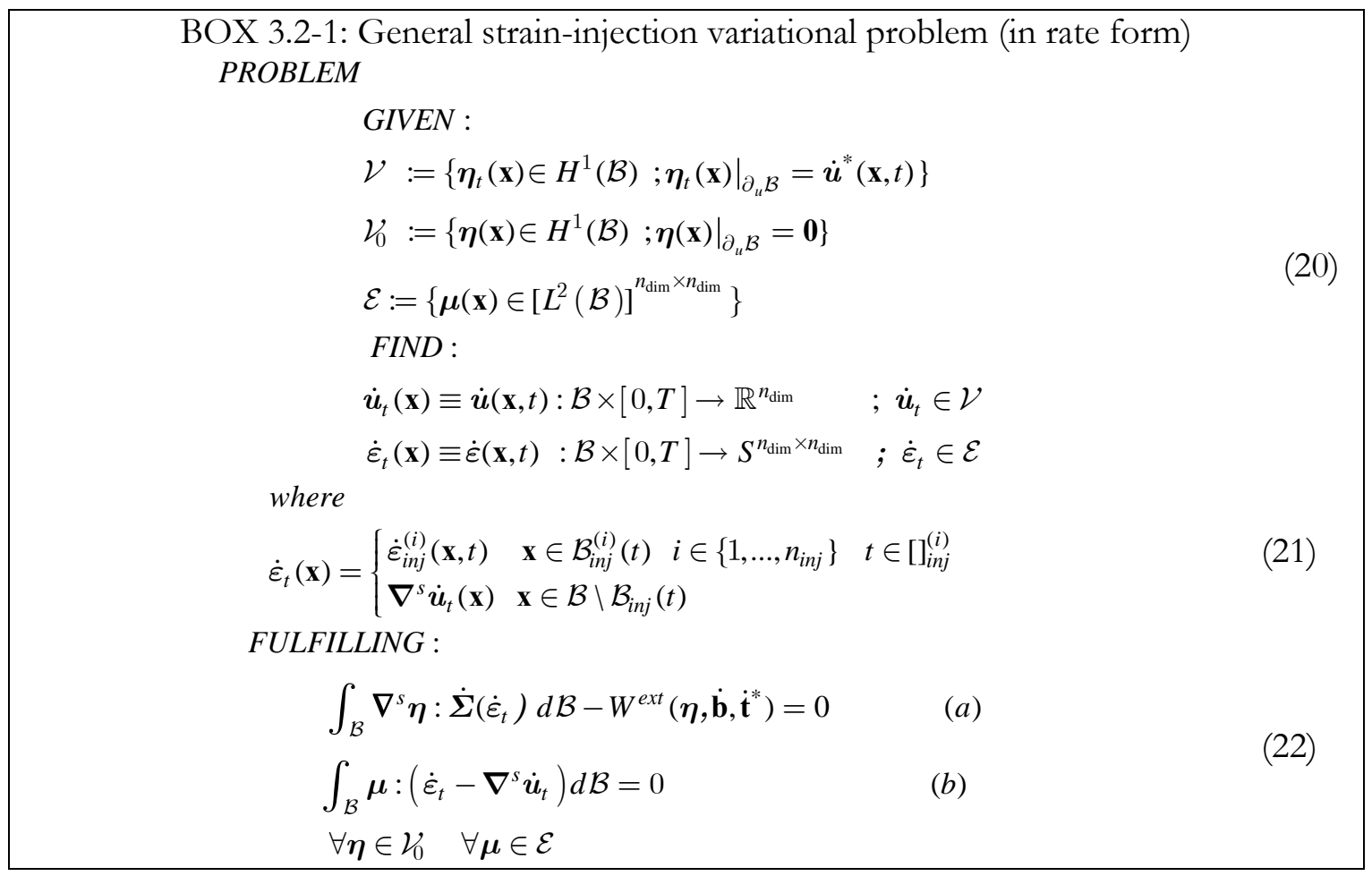

\subsection{Application to improving the strain-localization propagation capabilities.}

As a first illustration of the benefits provided by the proposed strain injection approach, let us consider the finite element discretization of $\mathcal{B}$, consisting in a $2 \mathrm{D}$ finite element mesh, of four-noded quadrilateral elements of typical size $h$, with $n_{\text {elem }}$ elements and $n_{\text {node }}$ nodes.

\subsubsection{A $u 1-\varepsilon 0$ mixed finite element formulation (full domain injection)}

Classical mixed finite element approaches can be considered as a sub-class of the straininjection techniques described in BOX 3.2-1, in which a unique injection domain $\left(n_{i n j}=1\right)$ 
occupying the whole mesh at all times is considered. In other words, mixed finite element formulations could be considered full domain injections with a stationary injection domain $\left(\mathcal{B}_{\text {inj }}=\mathcal{B}\right)$.

Let us now consider mixed, $u 1 / \varepsilon 0$, four-noded quadrilateral finite elements with linear interpolations of the displacements and element-wise constant strains, i.e:

$$
\begin{gathered}
\dot{\boldsymbol{u}}_{t}^{h}(\mathbf{x}) \equiv \dot{\boldsymbol{u}}^{h}(\mathbf{x}, t)=\sum_{i=1}^{n_{\text {mode }}} N_{i}(\mathbf{x}) \dot{\boldsymbol{u}}_{i}(t) ; \boldsymbol{\eta}^{h}(\mathbf{x})=\sum_{i=1}^{n_{\text {mode }}} N_{i}(\mathbf{x}) \boldsymbol{\eta}_{i} \\
\dot{\boldsymbol{\varepsilon}}_{t}^{h}(\mathbf{x}) \equiv \dot{\boldsymbol{\varepsilon}}^{h}(\mathbf{x}, t)=\sum_{e=1}^{n_{\text {elem }}} \phi^{(e)}(\mathbf{x}) \dot{\boldsymbol{\varepsilon}}^{(e)}(t) \quad ; \boldsymbol{\mu}^{h}(\mathbf{x})=\sum_{e=1}^{n_{\text {elem }}} \phi^{(e)}(\mathbf{x}) \boldsymbol{\mu}^{(e)}
\end{gathered}
$$

where $N_{i}$ stands for the standard bi-linear shape functions associated to node $i$, and $\boldsymbol{u}_{i}$ and $\varepsilon^{(e)}$ stand, respectively, for the corresponding nodal displacement and elemental strain degrees of freedom ${ }^{5}$. The element-wise-constant function $\phi^{(e)}$ is defined to have its support on the element $\mathcal{B}^{(e)}$, i.e.:

$$
\phi^{(e)}(\mathbf{x})=\left\{\begin{array}{lll}
1 & \text { if } & \mathbf{x} \in \mathcal{B}^{(e)} \\
0 & \text { if } & \mathbf{x} \notin \mathcal{B}^{(e)}
\end{array}\right.
$$

Considering the previous finite element approximations, at a given time $t$, equations (22) read:

$$
\begin{aligned}
& \left.\sum_{e=1}^{n_{\text {elem }}} \int_{\mathcal{B}^{(e)}} \nabla^{s} \boldsymbol{\eta}^{h}(\mathbf{x}): \dot{\boldsymbol{\Sigma}}\left(\dot{\boldsymbol{\varepsilon}}^{(e)}\right) d \mathcal{B}=W^{e x t}\left(\boldsymbol{\eta}^{h}, \dot{\mathbf{b}}, \dot{\mathbf{t}}^{*}\right) \quad \forall \boldsymbol{\eta}^{h} \in \mathcal{V}_{0}^{h} \quad a\right) \\
& \int_{\mathcal{B}}(e) \\
& \left.\boldsymbol{\mu}^{(e)}\left(\dot{\boldsymbol{\varepsilon}}^{(e)}-\nabla^{s} \dot{\boldsymbol{u}}^{h}(\mathbf{x})\right) d \mathcal{B}=0 \quad \forall \boldsymbol{\mu}^{e} \quad e=1, \ldots, n_{\text {elem }} \quad b\right)
\end{aligned}
$$

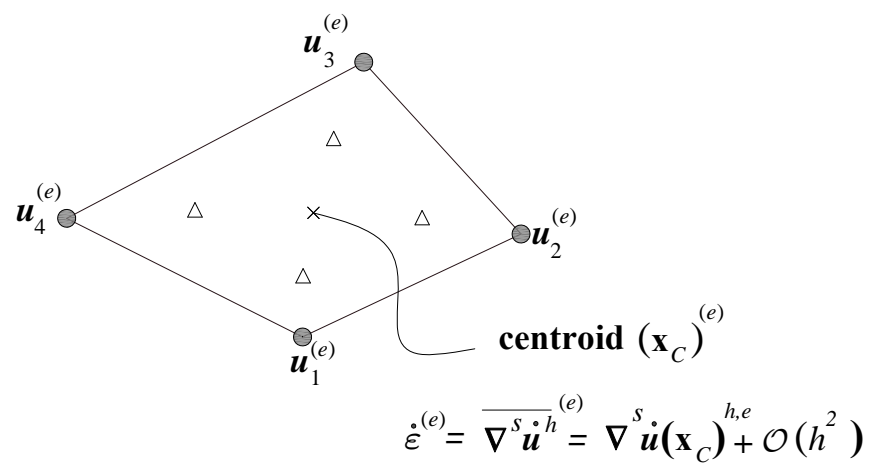

Figure 8. Quadrilateral finite element $(u 1 / \varepsilon 0)$ with bilinear displacements and constant strains.

Since the test functions, $\boldsymbol{\mu}^{h}(\mathbf{x})$, and the discretized strains, $\varepsilon^{h}(\mathbf{x}, t)$, in equation (24), are element-wise constant, equation (26-b) can be trivially solved, by condensing out $\dot{\varepsilon}^{(e)}$ at the element level, as:

$$
\dot{\boldsymbol{\varepsilon}}^{(e)}=\frac{\int_{B^{(}(e)} \boldsymbol{\nabla}^{s} \dot{\boldsymbol{u}}^{h}(\mathbf{x}) d \mathcal{B}}{\operatorname{meas}\left(\mathcal{B}^{(e)}\right)}={\overline{\nabla^{s} \dot{\boldsymbol{u}}^{h}}}^{(e)} \quad e=1, \ldots, n_{\text {elem }}
$$

where notation $\overline{(\cdot)}(e)$ stands for the spatial average of $(\cdot)$ on the element (e). Replacing equation (27) into equation (26)-(a) yields

${ }^{5}$ Sub-index $(\cdot)_{t}$ to indicate specification at time $t$ will be from now on omitted if not strictly necessary. 


$$
\begin{aligned}
& \sum_{e=1}^{n_{\text {elem }}} \int_{\mathcal{B}^{(e)}} \nabla^{s} \boldsymbol{\eta}^{h}(\mathbf{x}): \dot{\Sigma}\left({\overline{\nabla^{S} \dot{\boldsymbol{u}}^{h}}}^{(e)}\right) d \mathcal{B}= \\
= & \sum_{e=1}^{n_{\text {elem }}} \int_{\mathcal{B}^{(e)}}{\overline{\nabla^{s} \boldsymbol{\eta}^{h}}}^{(e)}: \dot{\boldsymbol{\Sigma}}\left({\overline{\nabla^{s} \dot{\boldsymbol{u}}^{h}}}^{(e)}\right) d \mathcal{B}=W^{e x t}\left(\boldsymbol{\eta}^{h}, \dot{\mathbf{b}}, \dot{\mathbf{t}}^{*}\right) \quad \forall \boldsymbol{\eta}^{h} \in \mathcal{V}_{0}^{h}
\end{aligned}
$$

REMARK 3.3-1. For the considered case of quadrilateral elements, the finite element implementation becomes simpler if the mean values, $\overline{(\cdot)}^{(e)}$, appearing in equation (28) are replaced, at the cost of a minor error ${ }^{6}$, by the value of the corresponding function at the centroid, $\mathbf{x}_{C}^{(e)}$, of the element (see Figure 8). This stems from the well-known equivalence of mixed formulations and selective/reduced formulations (Malkus and Hughes 1978).

\subsubsection{Stabilized mixed $u 1 / \varepsilon 0$ finite element formulation for quadrilaterals}

In view of REMARK 3.3-1, equation (28), displays the equivalence of the proposed $(u 1 / \varepsilon 0)$ mixed method with a reduced integration procedure of the displacement based formulation (sampling at the center of the element, (Malkus and Hughes 1978). This formulation is known to be unstable ${ }^{7}$, leading to zero energy (hourglass) displacement modes, which become dominant and pollute the solution in terms of the displacement field. Hourglass control techniques, with different theoretical foundations and stabilization procedures, have been proposed to overcome the instability provoked by reduced integration methods. [(Kosloff and Frazier 1978) (Belytschko and Bachrach 1986)]. Here the following stabilization term is proposed:

$$
\begin{aligned}
& \dot{W}^{s t a b}(t)=\sum_{e=1}^{n_{\text {elem }}} \dot{W}^{\text {stab }}(e)\left(\dot{\varepsilon}^{(e)}, \dot{\boldsymbol{u}}^{h}, t\right) \\
& \dot{W}^{\text {stab }}{ }^{(e)}\left(\dot{\boldsymbol{\varepsilon}}^{(e)}, \dot{\boldsymbol{u}}^{h}, t\right)=\tau^{(e)}(t) \int_{\mathcal{B}^{(e)}} \nabla^{s} \boldsymbol{\eta}^{h}(\mathbf{x}):\left[\dot{\boldsymbol{\Sigma}}\left(\dot{\boldsymbol{\varepsilon}}^{(e)}\right)-\dot{\boldsymbol{\Sigma}}\left(\nabla^{s} \dot{\boldsymbol{u}}^{h}(\mathbf{x})\right)\right] d \mathcal{B}= \\
& =\tau^{(e)}(t) \int_{\mathcal{B}^{(e)}} \overline{\nabla^{s} \boldsymbol{\eta}^{h}}: \dot{\Sigma}\left({\overline{\nabla^{s} \dot{\boldsymbol{u}}^{h}}}^{(e)}\right) d \mathcal{B}-\tau^{(e)}(t) \int_{\mathcal{B}^{(e)}} \nabla^{s} \boldsymbol{\eta}^{h}(\mathbf{x}): \dot{\boldsymbol{\Sigma}}\left(\nabla^{s} \dot{\boldsymbol{u}}^{h}(\mathbf{x})\right) d \mathcal{B}
\end{aligned}
$$

where equation (27) has been considered and $\tau^{(e)}(t) \in[0,1]$ is a stabilization parameter, which, in principle, can be specific for every element, $e$, and evolve along time. The stabilization term in equation (29) must be added to expression (28) as follows:

$$
\sum_{e=1}^{n_{e l e m}} \int_{\mathcal{B}^{(e)}}{\overline{\nabla^{s} \boldsymbol{\eta}^{h}}}^{(e)}: \dot{\boldsymbol{\Sigma}}\left({\overline{\nabla^{s} \dot{\boldsymbol{u}}^{h}}}^{(e)}\right) d \mathcal{B}=W^{e x t}\left(\boldsymbol{\eta}^{h}, \dot{\mathbf{b}}, \dot{\mathbf{t}}^{*}\right)^{e x t}+\dot{W}^{\text {stab }} \forall \boldsymbol{\eta}^{h} \in \mathcal{V}_{0}^{h}
$$

yielding

$$
\begin{aligned}
& \sum_{e=1}^{n_{\text {elem }}} \tau^{(e)}(t) \underbrace{\int_{\mathcal{B}^{(e)}} \nabla^{s} \boldsymbol{\eta}^{h}: \dot{\boldsymbol{\Sigma}}\left(\nabla^{s} \dot{\boldsymbol{u}}^{h}(\mathbf{x})\right) d \mathcal{B}}_{\begin{array}{c}
\text { full integration } \\
\text { (irreducible) form }
\end{array}}+ \\
& +\sum_{e=1}^{n_{\text {elem }}}\left(1-\tau^{(e)}(t)\right) \underbrace{\int_{\mathcal{B}^{(e)}}{\overline{\nabla^{s} \boldsymbol{\eta}^{h}}}^{(e)}: \dot{\boldsymbol{\Sigma}}\left({\overline{\nabla^{s} \dot{\boldsymbol{u}}^{h}}}^{(e)}\right) d \mathcal{B}}_{\begin{array}{c}
\text { reduced integration } \\
\text { (mixed) form }
\end{array}}=W^{\text {ext }}\left(\boldsymbol{\eta}^{h}, \dot{\mathbf{b}}, \dot{\mathbf{t}}^{*}\right) \forall \boldsymbol{\eta}^{h} \in \mathcal{V}_{0}^{h}
\end{aligned}
$$

\footnotetext{
${ }^{6}$ Due to the bilinear character of $\boldsymbol{\eta}^{h}(\mathbf{x})$ the committed error is $\mathcal{O}\left(h^{2}\right)$.

${ }^{7}$ From the point of view of the equivalent mixed formulation, the interpolation pairs bilinear displacementconstant stain, do not fulfill the inf-sup condition (Brezzi and Fortin 1991) and, therefore, the stability of the interpolated fields cannot be guaranted.
} 
The proposed stabilization method in equation (31) falls into the family of consistently stabilized methods (see [(Bochev and Gunzburger 2004; Dohrmann and Bochev 2004)]). The consistency of the stabilization stems from the fact that, from equation (26)-(b), the stabilization term, $\dot{W}^{\text {stab }}{ }^{(e)}$, in equation $(29)$ tends to zero with mesh refinement $(h \rightarrow 0$, $\dot{\varepsilon}^{(e)} \rightarrow \nabla^{s} \dot{u}^{h}$ ) regardless of the value of the stabilization parameter.

REMARK 3.3-2. The stabilized formulation in equation (31) can be regarded as a weighted combination of the irreducible displacement based formulation (fully integrated term) and the mixed displacement-strain formulation (reduced integrated term), weighted by $\tau^{(e)}$ and $\left(1-\tau^{(e)}\right)$ respectively. Analyzing expression (31), it is noticeable that, for $\tau^{(e)}=1 \forall e$, the method is equivalent to the standard irreducible formulation, whereas for, $\tau^{(e)}=0 \forall e$, the stabilization term vanishes and the unstable mixed formulation is recovered.

After some straightforward manipulations, equations (31) can be rearranged, in a more suitable form for implementation purposes, as:

$$
\begin{aligned}
& \sum_{e=1}^{n_{\text {elem }}} \int_{\mathcal{B}^{(e)}} \nabla^{s} \boldsymbol{\eta}^{h}: \dot{\tilde{\sigma}}_{t}^{(e)}(\mathbf{x}) d \mathcal{B}=W_{t}^{e x t}\left(\boldsymbol{\eta}, \mathbf{b}_{t}(\mathbf{x}), \mathbf{t}_{t}^{*}(\mathbf{x})\right) \quad \forall \boldsymbol{\eta}^{h} \in \mathcal{V}_{0}^{h} \quad \forall t \in[0, T] \\
& \dot{\boldsymbol{\sigma}}_{t}^{(e)}(\mathbf{x})=\tau^{(e)}(t) \dot{\boldsymbol{\Sigma}}\left(\nabla^{s} \dot{\boldsymbol{u}}_{t}^{h}(\mathbf{x})\right)+\left(1-\tau^{(e)}(t)\right) \dot{\boldsymbol{\Sigma}}\left({\overline{\nabla^{s}} \dot{\boldsymbol{u}}_{t}^{h}(\mathbf{x})}^{(e)}\right)
\end{aligned}
$$

where $\tilde{\sigma}_{t}^{(e)}(\mathbf{x}) \equiv \vec{\sigma}^{(e)}(\mathbf{x}, t)$ are termed the stabilized stresses. The described setting has a conceptually simple implementation in the context of a discretization of the time interval $[0, T]$. For the time sub-interval $\left[t_{n}, t_{n+1}\right] \subset[0, T]$ such that $\Delta t=t_{n+1}-t_{n}$, implicit integration of equations (31), yields to the following form:

$$
\begin{aligned}
& \sum_{\mathcal{B}^{(e)} \in \mathcal{B}} \int_{\mathcal{B}^{(e)}} \nabla^{s} \eta^{h}: \breve{\sigma}_{n+1}^{(e)} d \mathcal{B}=W_{n+1}^{e x t} \forall \boldsymbol{\eta}^{h} \in \mathcal{V}_{0}^{h} \\
& \breve{\boldsymbol{\sigma}}_{n+1}^{(e)}=\breve{\boldsymbol{\sigma}}_{n}^{(e)}(\mathbf{x})+\Delta \breve{\boldsymbol{\sigma}}_{n+1}^{(e)}(\mathbf{x}) \\
& \Delta \breve{\boldsymbol{\sigma}}_{n+1}^{(e)}(\mathbf{x})=\tau_{n+1}^{(e)} \underbrace{\left(\Sigma\left[\nabla^{s} \boldsymbol{u}_{n+1}^{h}(\mathbf{x})\right]-\boldsymbol{\sigma}_{n}^{(e)}(\mathbf{x})\right)}_{\Delta \boldsymbol{\sigma}_{n+1}^{(e)}(\mathbf{x})}+\left(1-\tau_{n+1}^{(e)}\right) \underbrace{\left(\Sigma\left(\bar{\nabla}^{s} \boldsymbol{u}_{n+1}^{h}\right)^{(e)}-\overline{\boldsymbol{\sigma}}_{n}^{(e)}\right)}_{\Delta \overline{\boldsymbol{\sigma}}_{n+1}^{(e)}}
\end{aligned}
$$

Notice that this version preserves the typical structure of the residual force vector in standard non-linear finite element codes, where the regular stresses, $\sigma_{n+1}^{(e)}(\mathbf{x})$ are substituted by the stabilized stresses $\boldsymbol{\sigma}_{n+1}^{(e)}(\mathbf{x})$. Hence, the integral in equation (33)-(a) can be evaluated, for the considered quadrilateral element, by a standard quadrature, for example using a $2 \times 2$ Gauss integration rule. The specific nuance is that, in addition to the regular stresses $\boldsymbol{\sigma}_{n+1}^{(e)}(\mathbf{x})=\boldsymbol{\Sigma}\left(\nabla^{s} \boldsymbol{u}_{n+1}^{h}(\mathbf{x})\right)$, the stabilized stresses, $\boldsymbol{\sigma}_{n+1}^{(e)}(\mathbf{x})$, should be computed and stored at the regular Gauss points, and the elemental-wise constant stresses, $\overline{\boldsymbol{\sigma}}_{n+1}^{(e)}=\boldsymbol{\Sigma}\left({\overline{\nabla^{s} \boldsymbol{u}_{n+1}^{h}(\mathbf{x})}}^{(e)}\right)$, should be also computed, and then possibly stored, at an additional sampling point ${ }^{8}$ (see REMARK 3.3-1).

The summary of the formulation for implementation purposes is given in BOX 3.3-1.

\footnotetext{
${ }^{8}$ This additional sampling point can be therefore regarded as a zero-weight integration point, just used for stress evaluation.
} 
BOX 3.3-1: Stabilized $u 1 / \varepsilon 0$ mixed finite element formulation for quadrilaterals (in rate PROBLEM form)

GIVEN :

$\mathcal{V}^{h}:=\left\{\boldsymbol{\eta}_{t}^{h}(\mathbf{x}) \in H^{1}(\mathcal{B}) ;\left.\boldsymbol{\eta}_{t}^{h}(\mathbf{x})\right|_{\partial_{u} \mathcal{B}}=\dot{\boldsymbol{u}}^{*}(\mathbf{x}, t)\right\}$

$\mathcal{V}_{0}^{h}:=\left\{\boldsymbol{\eta}^{h}(\mathbf{x}) \in H^{1}(\mathcal{B}) ;\left.\boldsymbol{\eta}^{h}(\mathbf{x})\right|_{\partial_{u} \mathcal{B}}=\mathbf{0}\right\}$

FIND :

$\dot{u}_{t}^{h}(\mathbf{x}) \equiv \dot{u}^{h}(\mathbf{x}, t): \mathcal{B} \times[0, T] \rightarrow \mathbb{R}^{n_{\mathrm{dim}}} \quad ; \dot{\boldsymbol{u}}_{t}^{h} \in \mathcal{V}^{h}$

FULFILLING :

$$
\begin{aligned}
& \sum_{\mathcal{B}^{(e)} \in \mathcal{B}} \int_{\mathcal{B}^{(e)}} \nabla^{s} \boldsymbol{\eta}^{h}: \breve{\boldsymbol{\sigma}}_{n+1}^{(e)} d \mathcal{B}=W_{n+1}^{e x t} \quad \forall \boldsymbol{\eta}^{h} \in \mathcal{V}_{0}^{h} \\
& \widetilde{\boldsymbol{\sigma}}_{n+1}^{(e)}(\mathbf{x})=\widetilde{\boldsymbol{\sigma}}_{n}^{(e)}(\mathbf{x})+\Delta \widetilde{\boldsymbol{\sigma}}_{n+1}^{(e)}(\mathbf{x}) \\
& \Delta \breve{\boldsymbol{\sigma}}_{n+1}^{(e)}(\mathbf{x})=\tau_{n+1}^{(e)} \underbrace{\left(\boldsymbol{\sigma}_{n+1}^{(e)}(\mathbf{x})-\boldsymbol{\sigma}_{n}^{(e)}(\mathbf{x})\right)}_{\Delta \boldsymbol{\sigma}_{n+1}^{(e)}(\mathbf{x})}+\left(1-\tau_{n+1}^{(e)}\right) \underbrace{\left(\overline{\boldsymbol{\sigma}}_{n+1}^{(e)}-\overline{\boldsymbol{\sigma}}_{n}^{(e)}\right)}_{\Delta \bar{\sigma}_{n+1}^{(e)}} \\
& \boldsymbol{\sigma}_{n+1}^{(e)}(\mathbf{x})=\boldsymbol{\Sigma}\left(\nabla^{s} \boldsymbol{u}_{n+1}^{h}(\mathbf{x})\right) \\
& \overline{\boldsymbol{\sigma}}_{n+1}^{(e)}=\boldsymbol{\Sigma}\left({\overline{\nabla^{s} u_{n+1}^{h}(\mathbf{x})}}^{(e)}\right) \approx \boldsymbol{\Sigma}\left(\nabla^{s} u_{n+1}^{h}\left(\mathbf{x}_{C}^{(e)}\right)\right)
\end{aligned}
$$

From now on, sub-indices $(\cdot)_{n},(\cdot)_{n+1}$, to indicate evaluation at the time sub-interval bounds, will be omitted when not strictly necessary.

\subsubsection{Evolving injection of an element-wise-constant strain mode in quadrilaterals, for strain localization problems}

Let us now consider a specific strain-injection scenario with one injection domain $\left(n_{\text {inj }}=1\right)$ but, this time $\mathcal{B}_{\text {inj }}(t) \subset \mathcal{B}$. The injection domain, $\mathcal{B}_{\text {inj }}$, evolves along time ( $\left.\mathcal{B}_{\text {inj }}(t) \neq \varnothing \forall t \in[]_{i n j} \subset[0, T]\right)$ and it is defined through:

$$
\left.\mathcal{B}_{\text {inj }}(t):=\left\{\mathbf{x} \in \mathcal{B} \mid t \geq t_{B}(\mathbf{x}) ; \dot{\alpha}(\mathbf{x}, t)>0\right)\right\}
$$

where $t_{B}(\mathbf{x})$ is the so-called bifurcation time computed trough the discontinuous material bifurcation problem ${ }^{9}$ (Oliver, Huespe et al. 2012), which reads:

PROBLEM

GIVEN : $\mathcal{C}^{(\text {load })}(\mathbf{x}, t)=\frac{\partial \Sigma(\varepsilon(\mathbf{x}, t))}{\partial \varepsilon(\mathbf{x}, t)} ; t \in[0, T]$

FIND: the first $t \equiv t_{B}(\mathbf{x}) \in[0, T]$ and $\mathbf{n}(\mathbf{x}) \in \mathbb{I}:=\left\{\mathbf{v} \in \mathbb{R}^{n_{\mathrm{dim}}} \mid\|\mathbf{v}\|=1\right\}$;

FULFILLING :

$$
\mathbf{Q}_{\text {loc, } t_{B}}(\mathbf{n}) \cdot \mathbf{m}=\left[\mathbf{n} \cdot \mathcal{C}^{(\text {load })}\left(\mathbf{x}, t_{B}\right) \cdot \mathbf{n}\right] \cdot \mathbf{m}=\mathbf{0} \quad \text { for some } \mathbf{m} \in \mathbb{I}
$$

In equation (36), $\mathcal{C}^{(\text {load })}$ stands for the in-loading tangent constitutive operator of the chosen constitutive model, described as $\sigma(\mathbf{x}, t)=\boldsymbol{\Sigma}(\varepsilon(\mathbf{x}, t))$, and $\mathbf{Q}_{\text {loc }}=\left[\mathbf{n} \cdot \mathcal{C}^{(\text {load })} \cdot \mathbf{n}\right]$ is the so-called localization tensor (sometimes also termed the acoustic tensor), computed in terms of the bifurcation direction described by the unit vector field, $\mathbf{n}(\mathbf{x})$. More information on obtaining analytical and numerical solutions of the discontinuous material bifurcation problem in equation (36) can be found in references (Oliver and Huespe 2004a; Oliver, Huespe et al. 2010)

\footnotetext{
${ }^{9}$ The bifurcation time, $t_{B}(\mathbf{x})$, signals the first time that the stress-strain constitutive equation is compatible with the appearance of a, regularized, displacement discontinuity in the considered material point, $\mathbf{x}$, thus triggering the strain localization.
} 
From equations (12), (35) and (36) the injection domain at a given time, $t$, here also termed the localization domain and denoted $\mathcal{B}_{l o c}(t) \equiv \mathcal{B}_{\text {inj }}(t)$, is defined as that set of elements whose barycenter has previously bifurcated $\left(t \geq t_{B}(\mathbf{x})\right)$ and is in in-loading regime $(\dot{\alpha}(\mathbf{x}, t)>0)$.

The motivation for this definition is rather intuitive: the aim of the proposed technique is to inject the elemental-wise constant (localized) strain mode (from now on shortened as CSM) only into the localizing elements at the current time $t$. On the other hand, the injection of the CSM implies the weak imposition of the geometric compatibility (see equations (14)-(b) and (21)) and this is expected to provide additional flexibility to the element improving its capacity to capture and propagate the strain localization.

The bifurcated points are the points amenable to develop a localization of a strain field, and only those elements having a bifurcated centroid are injected according to equation (35). The reason for injecting only those bifurcated elements, and not all the elements of the mesh, will be given in next sections.

Notice that when an element unloads $(\dot{\alpha}(\mathbf{x}, t)=0)$, according with the definition in equation (35) it leaves the injection domain $\mathcal{B}_{i n j}(t)$ and, therefore, this injection domain remains as small as possible while fulfilling equation (35).

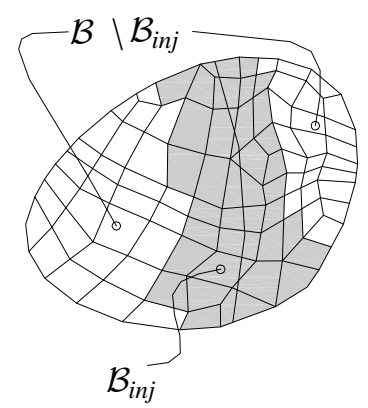

Figure 9. Injection domain in a strain localization problem.

The variational equations corresponding to the finite element strain-injection problem (injection domain-restricted mixed $\mathbf{u} / \varepsilon$ formulation), in equation (26), read as follows:

$$
\begin{aligned}
& \int_{\mathcal{B} \backslash \mathcal{B}_{i n j}} \nabla^{s} \boldsymbol{\eta}^{h}: \dot{\boldsymbol{\Sigma}}\left(\nabla^{s} \dot{\boldsymbol{u}}^{h}\right) d \mathcal{B}+\sum_{\forall \mathcal{B}^{(e)} \subset \mathcal{B}_{i n j}(t)} \int_{\mathcal{B}^{(e)}} \nabla^{s} \boldsymbol{\eta}^{h}: \dot{\boldsymbol{\Sigma}}\left(\dot{\varepsilon}_{i n j}^{(e)}\right) d \mathcal{B}=W^{e x t}\left(\boldsymbol{\eta}, \dot{\mathbf{b}}, \dot{\mathbf{t}}^{*}\right) \quad \forall \boldsymbol{\eta}^{h} \in \mathcal{V}_{0}^{h} \\
& \int_{\mathcal{B}}(e) \boldsymbol{\mu}^{(e)}:\left(\dot{\varepsilon}_{i n j}^{(e)}-\nabla^{s} \dot{\boldsymbol{u}}^{h}(\mathbf{x})\right) d \mathcal{B}=0 \quad \forall \boldsymbol{\mu}^{e} \quad \forall \mathcal{B}^{(e)} \subset \mathcal{B}_{i n j}(t)
\end{aligned}
$$

where $\dot{\varepsilon}_{i n j}^{(e)}$ is the injected CSM field and $\boldsymbol{\mu}^{(e)}$ the corresponding (element-wise-constant) weighting field. Comparing equation (37) with equation (26), we notice that the kinematic equation (14)-(b) now is weakly imposed only for those elements belonging to the injection domain $\mathcal{B}_{\text {inj }}(t)$.

Repeating the process for solving the injected strains, in equations (26) to (28), one arrives to the counterpart of equation (28) for this injection case:

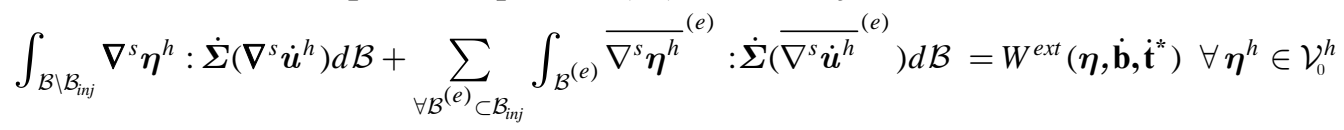

\subsubsection{Isochoric constitutive models - special treatment}

As commented above, the idea behind the injection concept in equations (37) is to inject specific strains at that part of the domain where strains localize, enhancing the performance of the corresponding finite elements as for localization and propagation of material failure. Intuitively, it seems then reasonable to define the domain of injection as the set of elements where discontinuous material bifurcation is detected. Nevertheless, when incompressible 
(e.g. $J_{2}$ plasticity) models are used, an additional difficulty appears due to the isochoric character of this constitutive equation. In fact, for this model, in earlier stages of the nonlinear loading, the irreducible formulation fully locks and the appropriated determination of the injection domain $\mathcal{B}_{\text {inj }}(t)$ in equation (35) becomes seriously affected.

Therefore, for this specific cases a slightly different strategy, combination of the techniques presented in sections 3.3.2. and 3.3.3 is proposed. The idea is to use as underlying element in $\mathcal{B} \backslash \mathcal{B}_{\text {inj }}$ the mixed/stabilized formulation in equations (33), endowed with an appropriated stabilization parameter, i.e. $\tau^{(e)}=\tau_{\text {stab }} \forall \mathcal{B}^{(e)} \in\left(\mathcal{B}^{(e)} \backslash \mathcal{B}_{\text {inj }}\right)^{10}$, so as to break the incompressibility locking and produce a good enough estimation of the injection domain. Then, the injection of the CSM in $\mathcal{B}_{\text {inj }}$ is based on the same scheme than in equation (38):

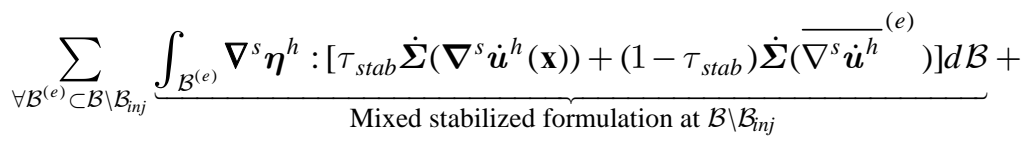

$$
\begin{aligned}
& +\sum_{\forall \mathcal{B}^{(e)} \subset \mathcal{B}_{\text {inj }}} \underbrace{\int_{\mathcal{B}^{(e)}} \nabla^{s} \boldsymbol{\eta}^{(e)}: \dot{\boldsymbol{\Sigma}}\left(\bar{\nabla}^{s} \dot{\boldsymbol{u}}^{(e)}\right.}_{\text {Injection of CSM at } \mathcal{B}_{\text {inj }}}) d \mathcal{B}=W^{e x t}\left(\boldsymbol{\eta}, \dot{\mathbf{b}}, \dot{\mathbf{t}}^{*}\right) \forall \boldsymbol{\eta}^{h} \in \mathcal{V}_{0}^{h}
\end{aligned}
$$

For the time sub-interval $\left[t_{n}, t_{n+1}\right] \subset[0, T]$ such that $\Delta t=t_{n+1}-t_{n}$, implicit integration of equations (39) yields, eventually, the algorithm in BOX 3.3-2 in terms of the effective stresses $\tilde{\sigma}_{n+1}^{(e)}$ (see Appendix A.1, for a proof in a more general case).

BOX 3.3-2: Time-discretized algorithm for injection of a constant-localized-strain-mode (CSM) considering a possible isochoric case

GIVEN : $\left.t_{n+1}, \mathbf{b}_{n+1}, \mathbf{t}_{n+1}^{*}, \tilde{\boldsymbol{\sigma}}_{n}^{(e)}(\mathbf{x}), \boldsymbol{\sigma}_{n}^{(e)}(\mathbf{x}), \overline{\boldsymbol{\sigma}}_{n}^{(e)}\right), \mathcal{B}_{i n j}\left(t_{n+1}\right)$

$$
\begin{aligned}
& \mathcal{\nu}^{h}=\left\{\boldsymbol{\eta}^{h}(\mathbf{x})=\sum_{1}^{n_{\text {node }}} N_{i}(\mathbf{x}) \boldsymbol{\eta}_{i} ; \boldsymbol{\eta}^{h}(\mathbf{x}) \in H^{1}\left(\mathcal{B}^{h}\right) ;\left.\boldsymbol{\eta}^{h}(\mathbf{x})\right|_{\partial_{u} \mathcal{B}^{h}}=\boldsymbol{u}^{*}\left(\mathbf{x}, t_{n+1}\right)\right\} \\
& \mathcal{V}_{0}^{h}=\left\{\boldsymbol{\eta}^{h}(\mathbf{x})=\sum_{1}^{n_{\text {node }}} N_{i}(\mathbf{x}) \boldsymbol{\eta}_{i} ; \boldsymbol{\eta}(\mathbf{x}) \in H^{1}\left(\mathcal{B}^{h}\right) ;\left.\boldsymbol{\eta}^{h}(\mathbf{x})\right|_{\partial_{u} \mathcal{B}^{h}}=\mathbf{0}\right\}
\end{aligned}
$$

FIND : $\boldsymbol{u}_{n+1}^{h}(\mathbf{x}) \equiv \boldsymbol{u}^{h}\left(\mathbf{x}, t_{n+1}\right) \in \mathcal{V}^{h}$

FULFILLING :

$$
\begin{aligned}
& \sum_{\mathcal{B}^{(e)} \in \mathcal{B}} \int_{\mathcal{B}^{(e)}} \nabla^{s} \boldsymbol{\eta}^{h}: \tilde{\boldsymbol{\sigma}}_{n+1}^{(e)} d \mathcal{B}=W_{n+1}^{e x t}\left(\boldsymbol{\eta}^{h}, \mathbf{b}_{n+1}, \mathbf{t}_{n+1}^{*}\right) \quad \forall \boldsymbol{\eta}^{h} \in \mathcal{V}_{0}^{h} \\
& \tilde{\boldsymbol{\sigma}}_{n+1}^{(e)}(\mathbf{x})=\tilde{\boldsymbol{\sigma}}_{n}^{(e)}(\mathbf{x})+\Delta \tilde{\boldsymbol{\sigma}}_{n+1}^{(e)}(\mathbf{x}) \\
& \Delta \tilde{\boldsymbol{\sigma}}_{n+1}^{(e)}(\mathbf{x})= \begin{cases}\tau_{n+1}^{(e)} \underbrace{\left(\boldsymbol{\sigma}_{n+1}^{(e)}(\mathbf{x})-\boldsymbol{\sigma}_{n}^{(e)}(\mathbf{x})\right)}_{\Delta \boldsymbol{\sigma}_{n+1}^{(e)}(\mathbf{x})}+\left(1-\tau_{n+1}^{(e)}\right) \underbrace{\left(\overline{\boldsymbol{\sigma}}_{n+1}^{(e)}-\overline{\boldsymbol{\sigma}}_{n}^{(e)}\right)}_{\Delta \overline{\boldsymbol{\sigma}}_{n+1}^{(e)}} & \mathcal{B}^{(e)} \subset \mathcal{B} \backslash \mathcal{B}_{i n j} \\
\underbrace{\left(\overline{\boldsymbol{\sigma}}_{n+1}^{(e)}-\overline{\boldsymbol{\sigma}}_{n}^{(e)}\right)}_{\Delta \overline{\boldsymbol{\sigma}}_{n+1}^{(e)}} & \mathcal{B}^{(e)} \subset \mathcal{B}_{i n j}\end{cases} \\
& \boldsymbol{\sigma}_{n+1}^{(e)}(\mathbf{x})=\boldsymbol{\Sigma}\left(\nabla^{s} \boldsymbol{u}_{n+1}^{h}(\mathbf{x})\right) \\
& \overline{\boldsymbol{\sigma}}_{n+1}^{(e)}=\boldsymbol{\Sigma}\left({\overline{\nabla^{s} u_{n+1}^{h}(\mathbf{x})}}^{(e)}\right) \approx \boldsymbol{\Sigma}\left(\nabla^{s} \boldsymbol{u}_{n+1}^{h}\left(\mathbf{x}_{C}^{(e)}\right)\right) \\
& \tau_{n+1}^{(e)}=\tau_{\text {stab }} \rightarrow\left\{\begin{array}{l}
\tau_{\text {stab }}=0.1 \rightarrow \text { isochoric case } \\
\tau_{\text {stab }}=1.0 \rightarrow \text { general case }
\end{array}\right.
\end{aligned}
$$

\footnotetext{
${ }^{10}$ Parameter $\tau_{\text {stab }}$ is not of physical nature, but a stabilization parameter whose optimal value is obtained from numerical experimentation. In practice, good results have been obtained in all tested benchmarks by using $\tau_{\text {stab }}=0.1$ (no instabilities show up and the injection domains are properly determined).
} 


\subsubsection{Representative simulations}

\subsubsection{DCB test using an (stabilized) mixed $\boldsymbol{u}-\boldsymbol{\varepsilon}$ formulation}

Let us consider again the DCB test in Figure 6-(a). The reported experimental crack path follows a straight line (inclined $\alpha=19^{\circ}$ with the vertical axis). Therefore, vertically aligned meshes as the one in the figure will strongly challenge the standard finite element formulations, since the experimentally observed discontinuity path intersects the elements in directions not coincident with the mesh alignment.

The mixed formulation BOX 3.3-1 is now used to model the crack propagation. Figure 10 shows the localization pattern for different values of the stabilization parameter, assumed spatially constant, $\tau_{\text {stab }}$.

Regarding the proposed stabilization method, it was mentioned in REMARK 3.3-2, that for values of $\tau_{\text {stab }} \equiv \tau$ approaching the unity (i.e. for $\tau \rightarrow 1.0$ ) the method is equivalent to the irreducible standard finite element approximation and therefore, the benefits inherent to the mixed formulation are expected to vanish. It is notorious the improvement on the element performance in terms of mesh bias independence for decreasing values of $\tau$. For $\tau=0.1$, it is shown the good agreement between the simulated crack path with that reported by experimental observation, while for $\tau=1.0$ the simulated crack is clearly affected by the vertical mesh alignment only zigzagging to the next raw after having propagated vertically a large number of elements.

(a) $\tau=1.0$

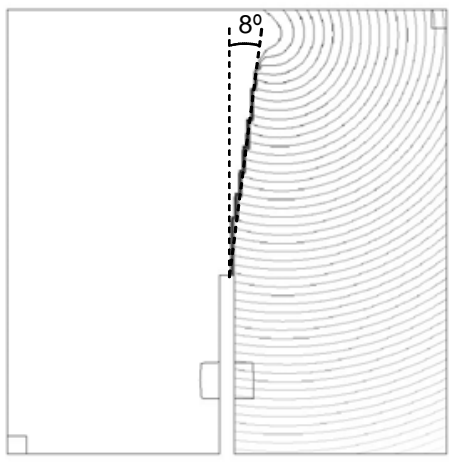

(b) $\tau=0.5$

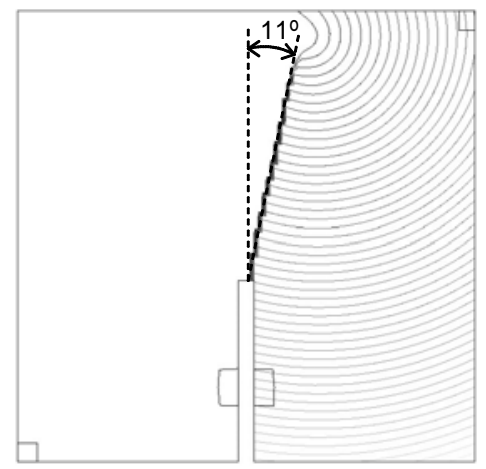

(c) $\tau=0.1$

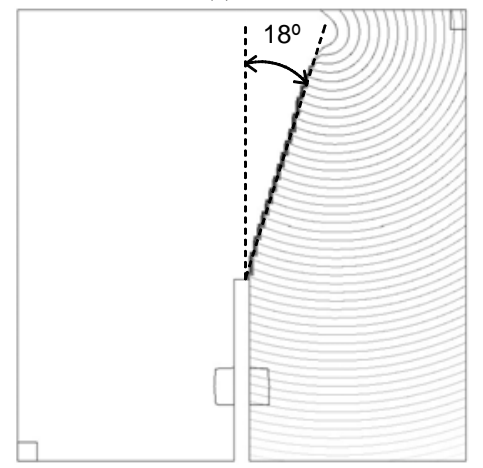

Figure 10. DCB test. Iso-displacement plots for different stabilization parameters $\tau$.

In view of these results, one could be tempted to decrease further the values of the stabilization parameter. For $\tau_{\text {stab }}=0$ the unstable mixed formulation is recovered and, on this view, for decreasing values of $\tau$ (i.e. for $\tau \rightarrow 0$ ), it is expected that instabilities show up. Figure 11 displays the numerical solutions obtained with $\tau=0.0001$.

(a)

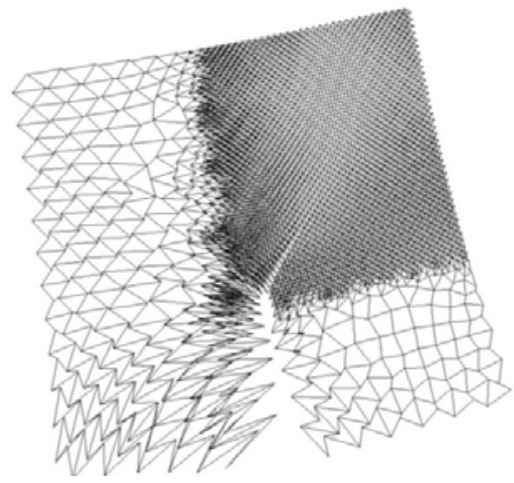

(b)

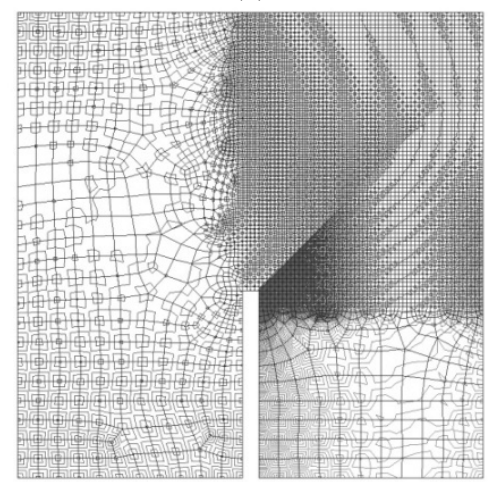

Figure 11. DCB test. Results for $\tau_{\text {stab }}=0.0001$ : (a) mesh deformation, (b) iso-displacement contours. 
It is clearly noticed the appearance of hourglass modes, dominating the solution in terms of the displacement field, which propagate across the mesh leading to a miss predicted collapse mechanism.

\subsubsection{DCB test using a CSM injection}

Now, results obtained with the injection of the CSM (BOX 3.3-2) are compared with those obtained with the stabilized mixed formulation in the previous section. It must be recalled that the CSM injection is a specific case in which the mixed formulation is restricted to the injection domain $\left(\mathcal{B}_{i n j}(t) \subset \mathcal{B}\right.$ see equation (35)), evolving along time and that no stabilization is performed. Therefore, the issue of the appearance of the hourglass modes remains in principle. However, results in Figure 12 show that no hourglass mode showsup (see Figure 11-(a)) and that the localization-band inclination matches the experimental one (see Figure 12-(b)).

(a)

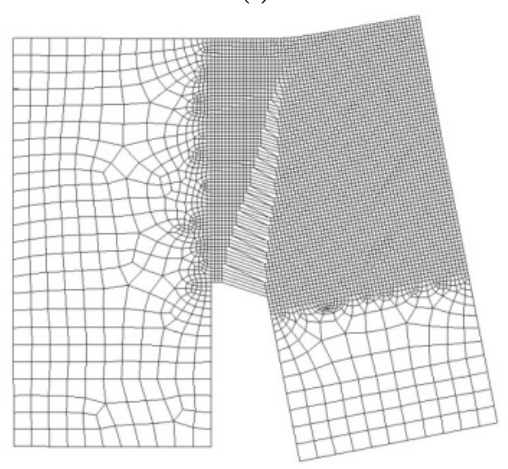

(b)

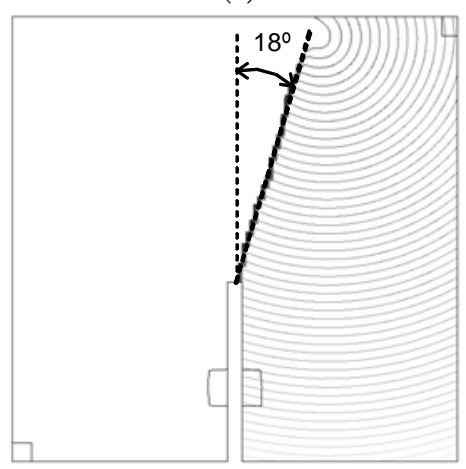

Figure 12. Results for the CSM injection strategy: (a) deformed mesh, (b) Iso-displacement plots.

For this non-homogeneous problem, in which the strain localization band evolves along time, it is interesting to see the evolution of the injection domain, $\mathcal{B}_{i n j}$. In Figure 13 this evolution is depicted for four representative sequential time steps.

(a) $\mathrm{t}=1$

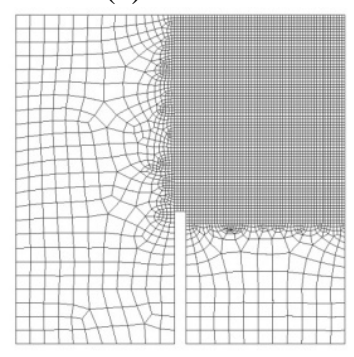

(b) $t=2$

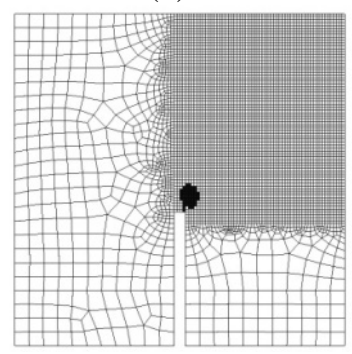

(c) $t=3$

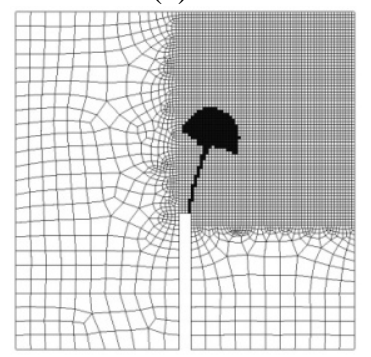

(d) $t=4$

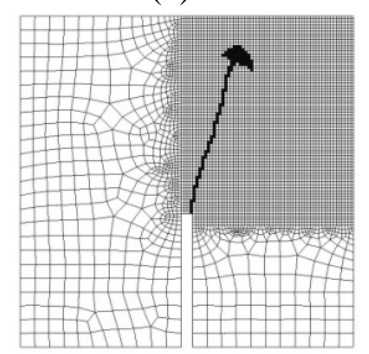

Figure 13. Evolution of the injection domain $\mathcal{B}_{\text {inj }}(t)$ (shaded zones) for different stages of the analysis.

Notice that, for initial stages of loading (Figure 13-(a)), the specimen behaves elastically (thus no injection domain is observed) and the irreducible standard formulation is applied in the entire body. For increasing loading, (Figure 13-(b)), some elements enter into a nonlinear regime and material bifurcation is detected. Those elements are then included in the injection domain, $\mathcal{B}_{i n j}$, trough the condition in equation (35), and, therefore, the CSM is injected into all them. It can be noticed the bulb-shaped character of the injection domain front, at the tip of the advancing localization band. Soon later (Figure 13-(c)-(d)), most of 
the bifurcated elements behind the bulb unload (i.e. $\dot{\alpha}=0$ ) returning to an elastic condition, so they leave the $\mathcal{B}_{\text {inj }}$ domain according to equation (35), except for an inclined band, behind the bulb, which remains in inelastic loading and defined the corresponding strain localization band.

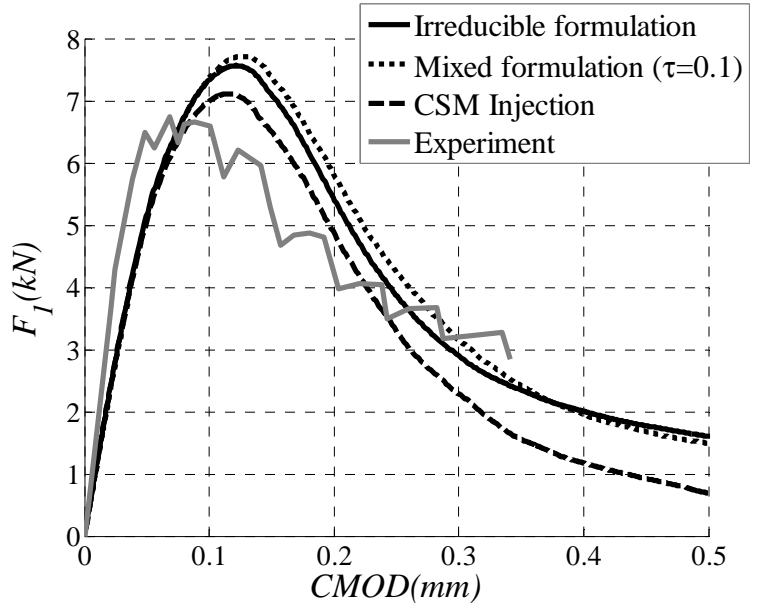

Figure 14.Curves $\mathrm{F}_{1}$ vs. Crack Mouth Opening Displacement (CMOD), obtained with the different procedures.

In Figure 14 quantitative responses for the different options are depicted in terms of the force-CMOD (crack-mouth-opening displacement) curves, together with the reported experimental results. None of the results matches perfectly the experimental curve ${ }^{11}$ but the interesting issue is the CSM injection produces the most flexible results (this being associated to a smaller dissipation and less stress-locking), and, thus, the smallest residual force values at the curve tail. This clearly indicates the benefits of the proposed technique in reducing the stress-locking effects.

\subsubsection{Isochoric problem. Homogeneous strip subjected to tensile stretching, in J2 plasticity.}

In order to rigorously assess the quantitative performance of the proposed technique, a simpler problem, for which an exact analytical solution is available, is necessary. This is the case of the problem sketched in Figure 15.

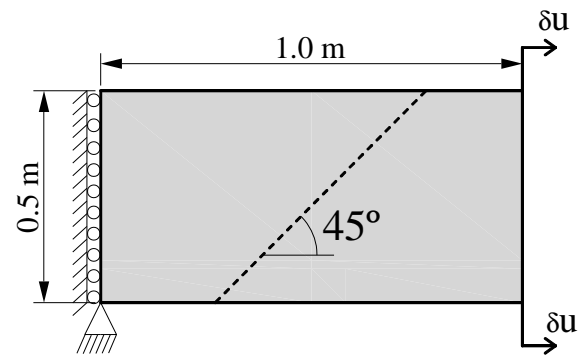

\begin{tabular}{cccc}
\hline $\begin{array}{c}\sigma_{y} \\
{[\mathrm{kPa}]}\end{array}$ & $\begin{array}{c}E \\
{[\mathrm{kPa}]}\end{array}$ & $v$ & $\begin{array}{c}G_{f} \\
{[\mathrm{~N} / \mathrm{m}]}\end{array}$ \\
\hline 1,0 & 120 & 0,49 & 22.7 \\
\hline
\end{tabular}

Figure 15.Strip subjected to tensile stretching using a $J_{2}$ plasticity model, where $\sigma_{y}$ stands for the yield stress, $E$ for the Young's modulus, $\nu$ for the Poisson's ratio and $G_{f}$ for the fracture energy.

The theoretical solution for this quasi-homogeneous problem (when it is slightly perturbed at some point, to break the initial homogeneity) consists of a straight slip line,

11 Neither no attempt was done to get a better fitting by tuning the material parameters of the model 
inclined $45^{\circ}$ and passing through the perturbed point. A rather coarse, unstructured, mesh of quadrilaterals is used to perform the tests as shown in Figure 16. Again, results with the mixed $(u / \varepsilon)$ stabilized formulation and with the injection of CSM techniques are compared.

(a) $\tau=1.0$

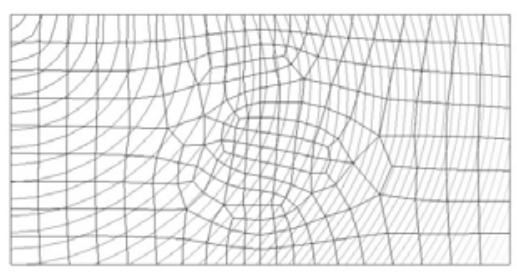

(c) $\tau=0.01$

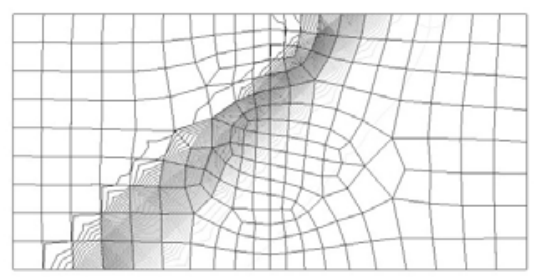

(b) $\tau=0.1$

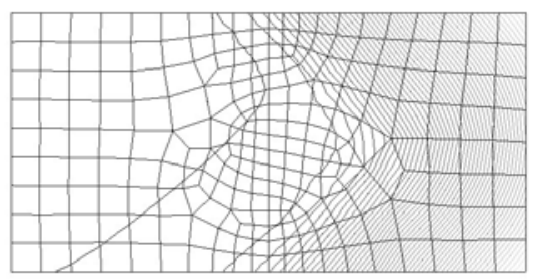

(d) $\tau=0.0001$

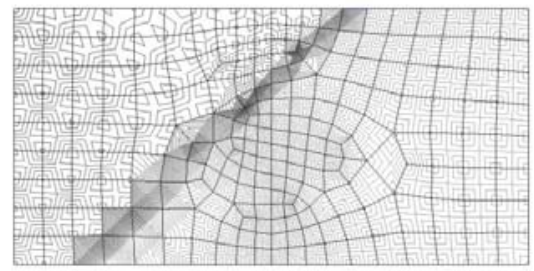

Figure 16. Strip subjected to tensile stretching using a $J_{2}$ plasticity model and mixed stabilized formulation. Iso-displacement contours obtained, for different values of $\tau$, at the final stage of analysis.

Figure 16 shows the localization patterns obtained with the mixed formulation and decreasing stabilization parameters. For the irreducible formulation $(\tau=1.0)$ the extreme locking of the problem does not allow any type of strain localization (Figure 16-(a)). To obtain a single-element-bandwidth localization band with the appropriated $45^{\circ}$ inclination, extremely small values of the stabilization parameter $(\tau=0.0001)$ have to be used, at the cost of evident instabilities and the appearance of hourglass modes polluting the solution (Figure 16-(d)).

(a)

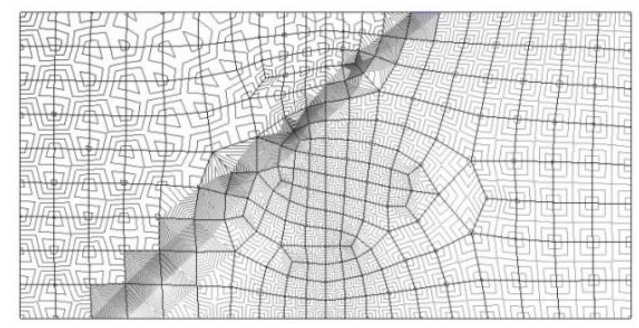

(b)

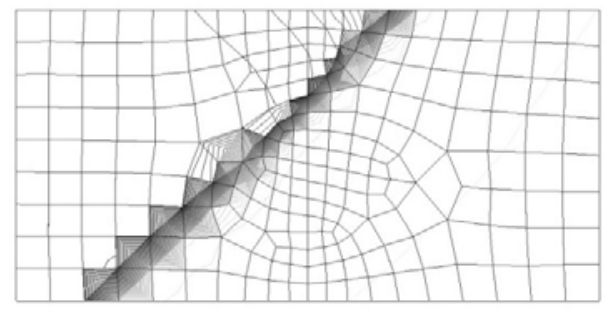

Figure 17. Strip subjected to tensile stretching using a $J_{2}$ plasticity model. Isodisplacement contours: (a) Mixed formulation $(\tau=0.0001$ ), (b) CSM injection.

Instead, using the CSM injection results become much better (see Figure 17-(b)). The CSM injection ${ }^{12}$ provides results that are equal or better than the best of the solutions with the mixed formulation but, this time, no type of instability is observed.

${ }^{12}$ In this case $\tau=\tau_{\text {stab }}=0.1$ in $\mathcal{B} \backslash \mathcal{B}_{\text {inj }}$. 
(a)

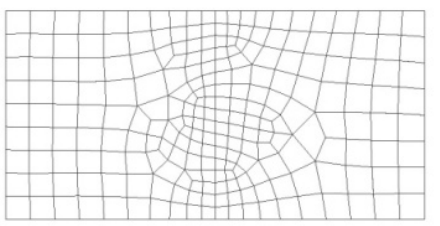

(b)

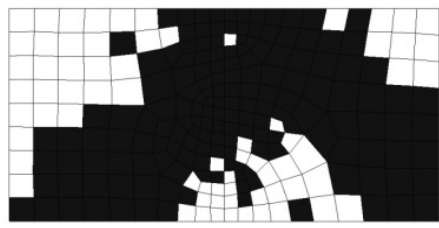

(c)

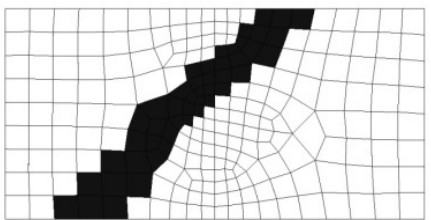

Figure 18. Strip subjected to tensile stretching using a $J_{2}$ plasticity model and CSM injection. Evolution of the injection domain, $\mathcal{B}_{i n j}(t)$, (shaded zones) at different stages of the analysis. (a) Elastic stage, (b) Immediately after the bifurcation stage, (c) final stage-

Figure 18, shows the evolution of the injection domain $\mathcal{B}_{\text {inj }}(t)$ at different stages of the analysis. It is remarkable that, at the bifurcation time, the injection domain appears as two bands inclined $\pm 45^{\circ}$, which correspond to the symmetric theoretical solution obtained from the bifurcation analysis of the homogeneous problem. The slight perturbation produced by the asymmetry of the mesh is then enough to break, immediately after bifurcation, the balance of the two bands (see Figure 18-(b)) in favor of one of them (Figure 18-(c)). This indicates the excellent propagation solution provided by the CSM injection technique.

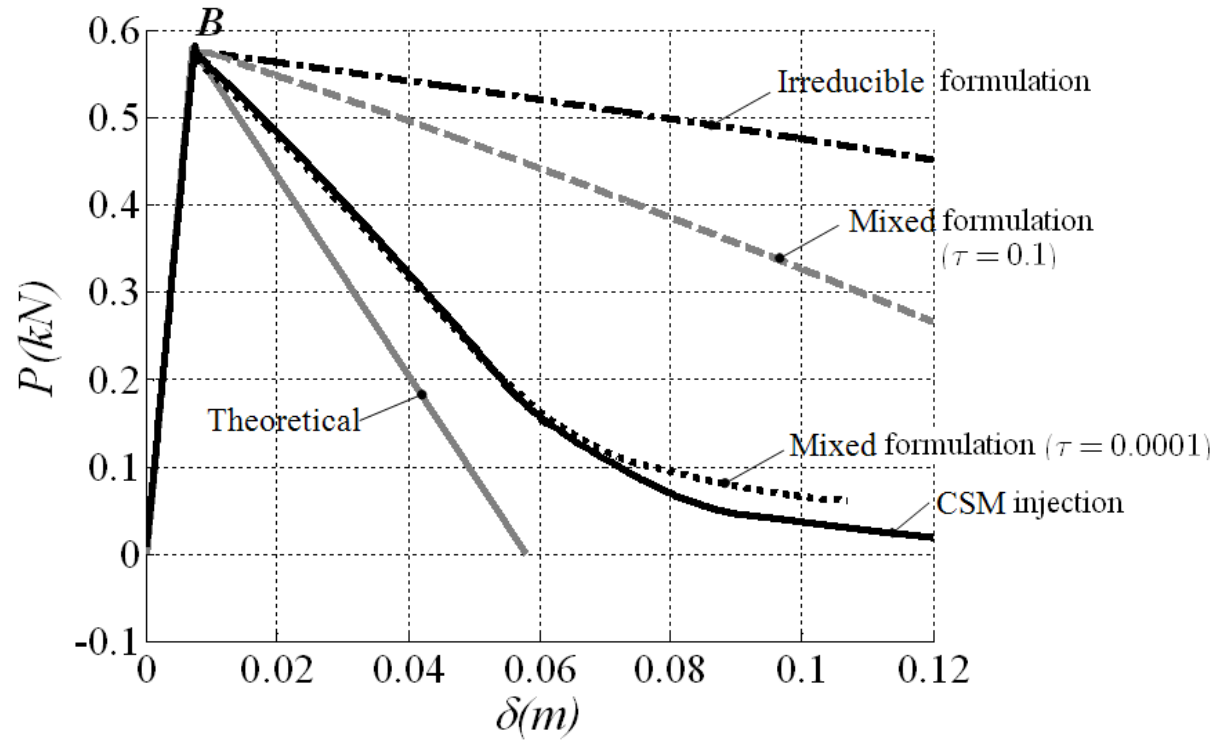

Figure 19. Strip subjected to tensile stretching. Force-displacement curves obtained with different techniques.

However, these promising qualitative results (in terms of propagation) do not translate into equally good quantitative results. For constant strain-softening modulus $(\mathcal{H}=\ell \overline{\mathcal{H}}$; $\left.\overline{\mathcal{H}}=\frac{1}{2}\left(\sigma_{y}\right)^{2} / G_{f}\right)$ the theoretical solution of the problem can be computed, and it is plotted in Figure 19 for the purposes of comparison with the considered cases.

Again, the benefits of the CSM injection technique, as for obtaining the most flexible solution, are evident. However, as it can be also checked in the figure, there is wide room for improvement with respect to the theoretical solution. Moreover, refinement of the mesh does not translate into substantial differences, and some degree of stress locking at the tail of the curve is noticeable even in the CSM case. This suggests further refinements in the strain injection technique presented in next sections. 


\section{Injection of propagating discontinuous displacement modes (DDM)}

In section 3 limited capabilities of the mixed finite element formulations, to reproduce the theoretical solutions of material failure/fracture problems were observed.

In terms of the qualitative correct propagation of the material failure, the CSM injection exhibits noticeable large improvements with respect to alternative (irreducible) formulations, this indicating that classical mesh-bias dependence flaws are almost totally overcome. However, the quantitative results, as compared in terms of action-response (force/displacement) curves, exhibit some mismatches when compared with those theoretical solutions, and non-negligible stress-locking effects are still noticed.

In a recent paper of the authors (Oliver, Huespe et al. 2012) a reason for these mismatches was identified and quantified in terms of error measures. The main conclusion of this work is that strain localization solutions, do not generally match fracture mechanics solutions. Excepting for very specific cases, typically finite element meshes specifically aligned with the (in general a-priori unknown) fracture path, they provide solutions exhibiting a relevant degree of mismatch with respect to the theoretical ones. This mismatch is caused by the limited ability of standard, or improved, finite elements to reproduce the strong discontinuity kinematics associated to a regularized strong discontinuity embedded into them, as it corresponds to fracture mechanics solutions. Indeed, the constant strain mode injection introduced in section 3.3.3 clearly provides extra flexibility to the element and largely enhance its propagation capabilities, but, on the other hand, the kinematics still exhibits a limited capability to describe a discontinuity in a one-element-bandwidth finite element band. In the ambit of the proposed injection techniques, the next natural improvement attempt is the injection of an additional strain mode, enhanced with the kinematics of a regularized displacement discontinuity, on those elements that capture the discontinuity. This additionally injected strain mode stems (in the limit of the regularization parameter) from a real discontinuous displacement field, and it will be therefore termed discontinuous displacement mode (DDM).

\subsection{Continuum Strong Discontinuity approach to material failure}

The continuum strong discontinuity approach (CSDA) to material failure was initially developed in the nineties (Simo, Oliver et al. 1993; Oliver 1996a) and subsequently, extended and used in a variety of applications by several authors (e.g. (Armero and Garikipati 1996; Alfaiate, Wells et al. 2002; Alfaiate 2003; Alfaiate, Simone et al. 2003; Mosler and Meschke 2003; Blanco, Huespe et al. 2006; Linero, Oliver et al. 2006; Linder and Armero 2007; Armero and Kim 2012). For the sake of completeness, the main elements of the CSDA, necessary for the central issues of this work, are described in next sections.

\subsubsection{Strong discontinuity kinematics}

Here we recall the basic ingredients of the strong discontinuity kinematics (Oliver and Huespe 2004b). Let us consider the continuum body, $\mathcal{B}$, (see Figure 20-(a)) split into two parts, $\mathcal{B}^{+}$and $\mathcal{B}^{-}$, by the strong discontinuity path, $\mathcal{S}$, across which the rate of displacement field, $\dot{u}(\mathbf{x})$, experiences a jump (strong discontinuity) $\llbracket \dot{u} \rrbracket=\left.\dot{u}\right|_{\mathbf{x} \in\left(\partial \mathcal{B}^{+} \cap \mathcal{S}\right)}-\left.\dot{u}\right|_{\mathbf{x} \in\left(\partial \mathcal{B}^{-} \cap \mathcal{S}\right)}$. 
(a)

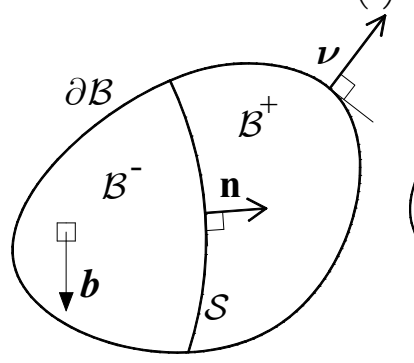

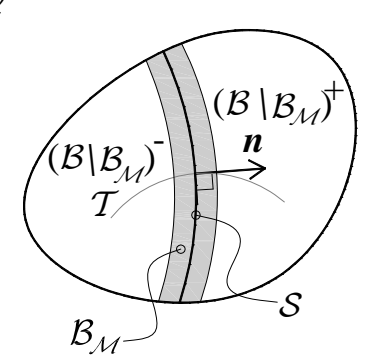

(b)

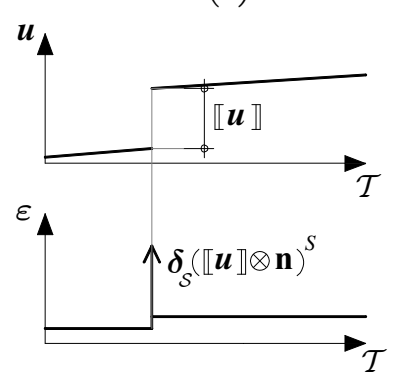

Figure 20 (a) Body exhibiting a strong discontinuity in the displacement field. (b) Strong discontinuity kinematics.

The mechanical description of the corresponding displacement field reads:

$$
\dot{u}=\dot{\bar{u}}+\mathcal{H}_{\mathcal{S}} \llbracket \dot{u} \rrbracket
$$

where $\dot{\bar{u}}$ stands for the smooth part of the (rate of) displacement field and $\mathcal{H}_{\mathcal{S}}$ is the Heaviside (step) function, shifted to $\mathcal{S}$. Due to computational reasons, related to the imposition of the essential boundary conditions, it is convenient to re-formulate equation (39) as the following equivalent expression (see (Oliver 1996b) for further details):

$$
\dot{\boldsymbol{u}}=\underbrace{\dot{\hat{\boldsymbol{u}}}-\varphi \llbracket \dot{\boldsymbol{u}} \rrbracket}_{\dot{\overline{\boldsymbol{u}}}}+\mathcal{H}_{\mathcal{S}} \llbracket \dot{\boldsymbol{u}} \rrbracket=\dot{\hat{\boldsymbol{u}}}+\underbrace{\left(\mathcal{H}_{\mathcal{S}}-\varphi\right)}_{\mathcal{M}_{S}} \llbracket \dot{\boldsymbol{u}} \rrbracket
$$

where $\dot{\hat{u}}$ is termed the generalized rate of displacement field, fulfilling the Dirichlet conditions of the problem, $\varphi(\mathbf{x})$ is the so-called indicatrix function, a continuous, in principle arbitrary, function fulfilling

$$
\varphi(\mathbf{x})=\left\{\begin{array}{l}
0 \quad \forall \mathbf{x} \in\left(\mathcal{B} \backslash \mathcal{B}_{\mathcal{M}}\right)^{-} \\
1 \quad \forall \mathbf{x} \in\left(\mathcal{B} \backslash \mathcal{B}_{\mathcal{M}}\right)^{+}
\end{array}\right.
$$

and $\mathcal{M}_{\mathcal{S}}(\mathbf{x})=\mathcal{H}_{\mathcal{S}}-\varphi(\mathbf{x})$ is the unit jump function, whose support is $\mathcal{B}_{\mathcal{M}}$ and exhibits a unit jump across $\mathcal{S}$, see Figure 20. The (infinitesimal) strain field corresponding to equation (42) reads:

$$
\dot{\boldsymbol{\varepsilon}}=\nabla^{S} \dot{\boldsymbol{u}}=\underbrace{\nabla^{S} \dot{\hat{\boldsymbol{u}}}+\left(\mathcal{M}_{\mathcal{S}} \otimes \nabla \llbracket \dot{\boldsymbol{u}} \rrbracket\right)^{S}-(\boldsymbol{\nabla} \varphi \otimes \llbracket \dot{\boldsymbol{u}} \rrbracket)^{S}}_{\hat{\dot{\varepsilon}}(\text { regular })}+\delta_{\mathcal{S}}(\mathbf{n} \otimes \llbracket \dot{\boldsymbol{u}} \rrbracket)^{S}=\hat{\dot{\varepsilon}}+\delta_{\mathcal{S}}(\mathbf{n} \otimes \llbracket \dot{\boldsymbol{u}} \rrbracket)^{S}
$$

where $\mathbf{n}$ is the unit vector orthogonal to $\mathcal{S}$ (pointing to $\mathcal{B}^{+}$).

\subsubsection{Boundary value problem}

In the context of the mechanical problem in Figure 20-(a), exhibiting strong discontinuities and the kinematics in equation (44), the original boundary value problem in equation (14) translates into the following one: 


\section{PROBLEM}

FIND: $\quad \dot{u}(\mathbf{x}, t): \mathcal{B} \times[0, T] \rightarrow \mathbb{R}^{n_{\text {dim }}}$

$$
\begin{aligned}
& \llbracket \dot{\boldsymbol{u}} \rrbracket(\mathbf{x}, t): \mathcal{B} \times[0, T] \rightarrow \mathbb{R}^{n_{\text {dim }}} \\
& \dot{\varepsilon}(\mathbf{x}, t): \mathcal{B} \times[0, T] \rightarrow \mathbb{S}^{n_{\text {dim }} \times n_{\text {dim }}} \\
& \dot{\boldsymbol{\sigma}}(\mathbf{x}, t): \mathcal{B} \times[0, T] \rightarrow \mathbb{S}^{n_{\text {dim }} \times n_{\text {dim }}}
\end{aligned}
$$

FULFILLING :

$$
\begin{aligned}
& \nabla \cdot \dot{\boldsymbol{\sigma}}+\dot{\mathbf{b}}=\mathbf{0} \\
& \forall \mathbf{x} \in \mathcal{B} \backslash \mathcal{S} \quad \rightarrow \text { equilibrium equation } \\
& \left\{\begin{array}{l}
\dot{\hat{\varepsilon}}(\mathbf{x}, t) \equiv \nabla^{S} \dot{\hat{\boldsymbol{u}}}+\left(\mathcal{M}_{\mathcal{S}} \otimes \nabla \llbracket \dot{\boldsymbol{u}} \rrbracket\right)^{S}-(\boldsymbol{\nabla} \varphi \otimes \llbracket \dot{\boldsymbol{u}} \rrbracket)^{S} \\
\dot{\varepsilon}(\mathbf{x}, t) \equiv \underbrace{\dot{\hat{\varepsilon}}(\mathbf{x}, t)}_{\text {(regular })}+\underbrace{\delta_{\mathcal{S}}(\mathbf{n} \otimes \llbracket \dot{\boldsymbol{u}} \rrbracket)^{S}}_{\text {singular }}
\end{array}\right. \\
& \dot{\boldsymbol{\sigma}}(\mathbf{x}, t)=\left\{\begin{array}{l}
\dot{\boldsymbol{\sigma}}_{\mathcal{B} \backslash \mathcal{S}}(\mathbf{x}, t) \equiv \dot{\boldsymbol{\Sigma}}(\dot{\hat{\varepsilon}}(\mathbf{x}, t)) \\
\dot{\boldsymbol{\sigma}}_{\mathcal{S}}(\mathbf{x}, t) \equiv \dot{\boldsymbol{\Sigma}}\left(\dot{\hat{\varepsilon}}(\mathbf{x}, t)+\delta_{\mathcal{S}}(\mathbf{n} \otimes \llbracket \dot{\boldsymbol{u}} \rrbracket)^{S}\right)
\end{array}\right. \\
& \forall \mathbf{x} \in \mathcal{B} \backslash \mathcal{S} \\
& \forall \mathbf{x} \in \mathcal{S} \\
& \rightarrow\left\{\begin{array}{c}
\text { compatibiliy equation } \\
\text { (strong discontinuity } \\
\text { kinematics) }
\end{array}\right.
\end{aligned}
$$

\subsubsection{A three field $(\dot{\hat{u}} / \dot{\bar{\varepsilon}} / \llbracket \dot{u} \rrbracket)$ variational problem}

Inspired in the boundary value problem of equations (45), let us now extend the two field, $\boldsymbol{u}-\boldsymbol{\varepsilon}$ variational problem in section 3.1 .2 to the following three-field, $\dot{\hat{\boldsymbol{u}}} / \dot{\bar{\varepsilon}} / \llbracket \dot{\boldsymbol{u}} \rrbracket$, problem (in rate form):

PROBLEM

GIVEN :

$$
\begin{aligned}
& \hat{\mathcal{V}}:=\left\{\boldsymbol{\eta}_{\boldsymbol{t}}(\mathbf{x}) \in\left[H^{1}(\mathcal{B})\right]^{n_{\mathrm{dim}}} ;\left.\boldsymbol{\eta}_{t}(\mathbf{x})\right|_{\partial_{u} \mathcal{B}}=\dot{\boldsymbol{u}}^{*}(\mathbf{x}, t)\right\} \\
& \hat{\mathcal{V}_{0}}:=\left\{\boldsymbol{\eta}(\mathbf{x}) \in H^{1}(\mathcal{B}) ;\left.\boldsymbol{\eta}(\mathbf{x})\right|_{\partial_{u} \mathcal{B}}=\mathbf{0}\right\} \\
& \hat{\mathcal{E}}:=\left\{\boldsymbol{\mu} \in\left[L^{2}(\mathcal{B})\right]^{n_{\text {dim }} \times n_{\text {dim }}}\right\} \\
& \tilde{\mathcal{U}}:=\left\{\boldsymbol{\beta} \in\left[L^{2}(\mathcal{B})\right]^{n_{\text {dim }}} ; \llbracket \boldsymbol{\beta} \rrbracket(\mathbf{x})=\mathbf{0} \forall \mathbf{x} \in \mathcal{S}\right\} \\
& \tilde{\Gamma}:=\left\{\tilde{\boldsymbol{\gamma}}=\chi_{\mathcal{S}}^{+}(\boldsymbol{\beta} \otimes \mathbf{n})^{S} ; \boldsymbol{\beta} \in \tilde{\mathcal{U}}\right\}
\end{aligned}
$$

\section{FIND}

$$
\begin{array}{ll}
\dot{\hat{u}}_{t}(\mathbf{x}) \equiv \dot{\hat{u}}(\mathbf{x}, t): \mathcal{B} \times[0, T] \rightarrow \mathbb{R}^{n_{\text {dim }}} & ; \quad \dot{\hat{u}}_{t} \in \hat{\mathcal{V}} \\
\dot{\hat{\varepsilon}}_{t}(\mathbf{x}) \equiv \dot{\hat{\varepsilon}}(\mathbf{x}, t): \mathcal{B} \times[0, T] \rightarrow \mathbb{S}^{n_{\text {dim }} \times n_{\text {dim }}} & ; \quad \dot{\varepsilon}_{t} \in \hat{\mathcal{E}} \\
\llbracket \dot{\boldsymbol{u}} \rrbracket_{t}(\mathbf{x}) \equiv \llbracket \dot{\boldsymbol{u}} \rrbracket(\mathbf{x}, t): \mathcal{B} \times[0, T] \rightarrow \mathbb{R}^{n_{\text {dim }}} \quad ; \quad \llbracket \dot{u} \rrbracket_{t} \in \tilde{\mathcal{U}}
\end{array}
$$




\section{FULFILLING}

$$
\begin{aligned}
& \int_{\mathcal{B}} \nabla^{s} \boldsymbol{\eta}: \dot{\boldsymbol{\sigma}}_{t} d \mathcal{B}-W^{\text {ext }}\left(\boldsymbol{\eta}, \dot{\mathbf{b}}, \dot{\mathbf{t}}^{*}\right)=0 \quad \forall \boldsymbol{\eta} \in \hat{\mathcal{V}_{0}} \\
& \left\{\begin{array}{l}
\int_{\mathcal{B} \backslash \mathcal{S}} \boldsymbol{\mu}:\left[\dot{\bar{\varepsilon}}_{t}-\dot{\hat{\varepsilon}}_{t}\left(\dot{\hat{\boldsymbol{u}}}_{t}, \llbracket \dot{\boldsymbol{u}} \rrbracket_{t}\right)\right] d \mathcal{B}=0 \quad \forall \boldsymbol{\mu} \in \hat{\mathcal{E}} \\
\dot{\hat{\varepsilon}}_{t}\left(\dot{\hat{\boldsymbol{u}}}_{t}, \llbracket \dot{\boldsymbol{u}} \rrbracket_{t}\right) \equiv \nabla^{S} \dot{\hat{\boldsymbol{u}}}_{t}(\mathbf{x})+\left(\mathcal{M} \mathcal{S} \otimes \nabla \llbracket \dot{\boldsymbol{u}} \rrbracket_{t}\right)^{S}-\left(\nabla \varphi \otimes \llbracket \dot{\boldsymbol{u}} \rrbracket_{t}\right)^{S} \\
\dot{\varepsilon}_{t}(\mathbf{x}, t)=\underbrace{\dot{\bar{\varepsilon}}_{t}(\mathbf{x})}_{(\text {regular })}+\underbrace{\delta_{\mathcal{S}}\left(\mathbf{n} \otimes \llbracket \dot{\boldsymbol{u}} \rrbracket_{t}\right)^{S}}_{\text {singular }} \\
\int_{\mathcal{B}} \tilde{\boldsymbol{\gamma}}: \dot{\boldsymbol{\sigma}}_{t} d \mathcal{B}=\int_{\mathcal{B}} \chi_{\mathcal{S}}^{+}(\boldsymbol{\beta} \otimes \mathbf{n})^{S}: \dot{\boldsymbol{\sigma}}_{t} d \mathcal{B}=\int_{\mathcal{S}} \boldsymbol{\beta} \cdot \llbracket \dot{\boldsymbol{\sigma}}_{t} \rrbracket_{\mathcal{S}}^{+} \cdot \mathbf{n} d \mathcal{S}=0 \quad \forall \tilde{\boldsymbol{\gamma}} \in \tilde{\Gamma} \\
\dot{\boldsymbol{\sigma}}(\mathbf{x}, t) \equiv \dot{\boldsymbol{\sigma}}_{t}(\mathbf{x})=\dot{\boldsymbol{\Sigma}}\left(\dot{\varepsilon}_{t}(\mathbf{x})\right)=\dot{\boldsymbol{\Sigma}}\left[\dot{\bar{\varepsilon}}_{t}+\delta_{\mathcal{S}}\left(\mathbf{n} \otimes \llbracket \dot{\boldsymbol{u}} \rrbracket_{t}\right)^{S}\right]
\end{array}\right.
\end{aligned}
$$

In equations (46) and (47), $\chi_{\mathcal{S}}^{+}$stands for a dipole-like generalized function ${ }^{13}$ defined through

$$
\left\{\begin{array}{l}
\int_{\mathcal{B}} \phi(\mathbf{x}) \chi_{\mathcal{S}}^{+} d \mathcal{B}=\int_{\mathcal{S}} \llbracket \phi \rrbracket_{\mathcal{S}}^{+}(\mathbf{x}) d \mathcal{S} \\
\left.\llbracket \phi \rrbracket_{\mathcal{S}}^{+}(\mathbf{x}) \equiv \phi(\mathbf{x})\right|_{\mathbf{x} \in\left(\partial \mathcal{B}^{+} \cap \mathcal{S}\right)}-\left.\phi(\mathbf{x})\right|_{\mathbf{x} \in \mathcal{S}} \quad \forall \mathbf{x} \in \mathcal{S}
\end{array}\right.
$$

for any, sufficiently regular, test function $\phi(\mathbf{x})$.

REMARK 4.1-1. Notice, from equation (48)-(c) that the stresses $\dot{\sigma}_{t}$ belong

to the space, $\tilde{\Gamma}^{\perp}$, orthogonal to the test space $\tilde{\Gamma}$, in equation (46), i.e.:

$$
\begin{aligned}
& \tilde{\Gamma}^{\perp}:=\left\{\boldsymbol{\tau} \in[\mathbb{S}]^{n_{\mathrm{dim}} \times n_{\mathrm{dim}}} ; \int_{\mathcal{B}} \chi_{\mathcal{S}}^{+}(\boldsymbol{\beta} \otimes \mathbf{n})^{S}: \boldsymbol{\tau} d \mathcal{B}=\int_{\mathcal{S}} \boldsymbol{\beta} \cdot \llbracket \boldsymbol{\tau} \mathbb{J}_{\mathcal{S}}^{+} \cdot \mathbf{n} d \mathcal{S}=0 \forall \boldsymbol{\beta} \in \tilde{\mathcal{U}}\right\} \\
& \Rightarrow \tilde{\Gamma}^{\perp}=\left\{\boldsymbol{\tau}(\mathbf{x}): \mathcal{B} \rightarrow \mathbb{S}^{n_{\operatorname{dim}} \times n_{\text {dim }}} ; \llbracket \boldsymbol{\tau} \rrbracket_{\mathcal{S}}^{+} \cdot \mathbf{n}=\mathbf{0} \forall \mathbf{x} \in \mathcal{S}\right\}
\end{aligned}
$$

Therefore, equation (47)-(c) implicitly imposes the traction $\dot{\sigma}_{t} \cdot \mathbf{n}$ to fulfill the $\mathcal{S}$ inner- traction continuity equation (45)-(e), i.e.:

$$
\llbracket \dot{\boldsymbol{\sigma}}_{t} \rrbracket_{\mathcal{S}}^{+} \cdot \mathbf{n}=\dot{\boldsymbol{\sigma}}_{\mathcal{B} \backslash \mathcal{S}}(\mathbf{x}, t) \cdot \mathbf{n}-\dot{\boldsymbol{\sigma}}_{\mathcal{S}}(\mathbf{x}, t) \cdot \mathbf{n}=\mathbf{0} \quad \forall \mathbf{x} \in \mathcal{S}
$$

Straightforward computations show that the solution of the variational problem in equations (47) corresponds to that of the B.V.P. in equations (45). More specifically, the variational equation (47)-(a) imposes the equilibrium equation (45)-(a), the $\mathcal{S}$-outer traction continuity equation (45)-(d) and the Dirichlet boundary equation (45)-(f). The variational equation (47)-(b) imposes the strong discontinuity kinematics in equation (45)-(b) and, finally, the variational equation (47)-(c) imposes the $\mathcal{S}$-inner traction continuity equation (45)-(e) (see REMARK 4.1-1). Finally, the constitutive equation (45)-(b) is imposed in strong form through equation (47)-(d).

REMARK 4.1-2. The previous formulation can be considered as a specific application of the Assumed Enhanced Strain Method (Simó and Rifai 1990) where the kinematically compatible strains, $\nabla^{S} \dot{\hat{\boldsymbol{u}}}_{t}(\mathbf{x})$, are enhanced by the strains, $\dot{\tilde{\varepsilon}}_{t}(\mathbf{x})$, such that (see equations (42) to (44)):

$$
\left\{\begin{array}{l}
\dot{\varepsilon}_{t}(\mathbf{x})=\nabla^{S} \dot{\hat{\boldsymbol{u}}}_{t}(\mathbf{x})+\dot{\tilde{\varepsilon}}_{t}(\mathbf{x}) \\
\dot{\tilde{\varepsilon}}_{t}(\mathbf{x})=\nabla^{S}\left(\mathcal{M}_{\mathcal{S}} \llbracket \dot{\boldsymbol{u}} \rrbracket_{t}(\mathbf{x})\right)=\underbrace{\left(\mathcal{M}_{\mathcal{S}} \otimes \nabla \llbracket \dot{\boldsymbol{u}} \rrbracket_{t}\right)^{S}-\left(\boldsymbol{\nabla} \varphi \otimes \llbracket \dot{\boldsymbol{u}} \rrbracket_{t}\right)^{S}+\delta_{\mathcal{S}}\left(\mathbf{n} \otimes \llbracket \dot{\boldsymbol{u}} \rrbracket_{t}\right)^{S}}_{\text {enhanced strains } \dot{\tilde{\varepsilon}}_{t}}
\end{array}\right.
$$

Therefore, the spaces of the enhanced strains, $\tilde{\mathcal{E}}$, and the test space, $\tilde{\Gamma}$, are (see equations (46) and (51)):

${ }^{13}$ returning, from its convolution over $\mathcal{B}$ with any function $\phi(\mathbf{x})$ (possibly discontinuous across $\mathcal{S}$ ), the integral of the jump $\llbracket \phi \rrbracket_{\mathcal{S}}^{+}(\mathbf{x})$ along the manifold $\mathcal{S}$. 


$$
\begin{aligned}
& \tilde{\mathcal{E}}:=\left\{\tilde{\varepsilon}=\nabla^{S}\left(\mathcal{M}_{\mathcal{S}} \boldsymbol{\beta}\right) \quad ; \boldsymbol{\beta} \in \tilde{\mathcal{U}}\right\} \\
& \tilde{\Gamma}:=\left\{\tilde{\gamma}=\chi_{\mathcal{S}}^{+}(\boldsymbol{\beta} \otimes \mathbf{n})^{S} ; \quad \boldsymbol{\beta} \in \tilde{\mathcal{U}}\right\}
\end{aligned}
$$

The fact that the functional space $\tilde{\mathcal{E}}$, where the enhanced strains $\dot{\tilde{\varepsilon}}_{t}$ in equation (51) live, and the test space $\tilde{\Gamma}$, where the functions testing the stresses $\dot{\sigma}_{t}$ in equation (47)-(c) live, are different, renders the variational setting unsymmetrical (Hughes 1987).

Also it should be noticed, from equations (48) and (52), that

$$
\int_{\mathcal{B}} \tilde{\gamma} d \mathcal{B}=\int_{\mathcal{B}} \chi_{\mathcal{S}}^{+}(\boldsymbol{\beta} \otimes \mathbf{n})^{S} d \mathcal{B}=\int_{\mathcal{S}} \llbracket(\boldsymbol{\beta} \otimes \mathbf{n})^{S} \rrbracket_{\mathcal{S}}^{+} d \mathcal{S}=\mathbf{0} \quad \forall \tilde{\gamma} \in \tilde{\Gamma}
$$

this satisfying the so-called patch test condition for assumed enhanced strain methods (Simó and Rifai 1990).

\subsection{Finite elements with embedded (regularized) displacement discontinuities}

Let us now consider the body $\mathcal{B}$ discretized in $2 \mathrm{D}$ mixed, $\hat{\boldsymbol{u}} 1 / \bar{\varepsilon} 0 / \llbracket \boldsymbol{u} \rrbracket 0$, four-noded quadrilateral finite elements $\mathcal{B}^{(e)}$, of typical size $h$ (see Figure 21).

(a)

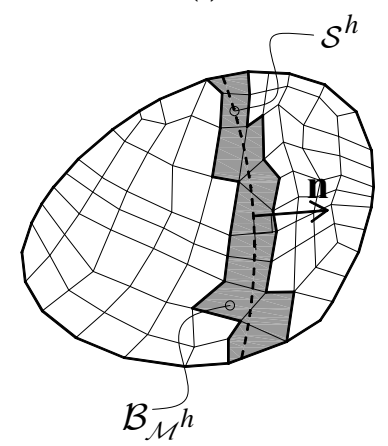

(b)

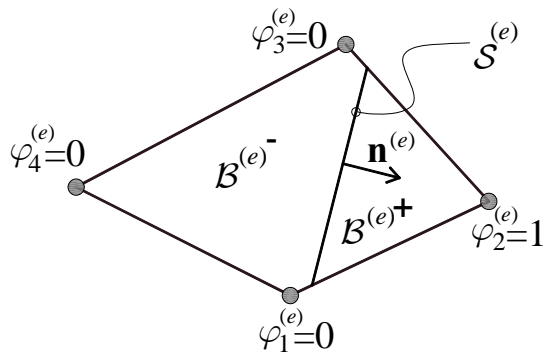

Figure 21 (a) Discretized domain crossed by displacement discontinuity path $\mathcal{S}^{h}$, (b) Finite element, $\mathcal{B}^{(e)}$ crossed by a displacement discontinuity path with elemental counterpart $\mathcal{S}^{(e)}$.

We shall consider linear interpolations of the displacements, and element-wise constant regular-strains and displacement-jumps, i.e.

$$
\begin{gathered}
\dot{\hat{\boldsymbol{u}}}^{h}(\mathbf{x}, t)=\sum_{i=1}^{n_{\text {node }}} N_{i}(\mathbf{x}) \dot{\hat{\boldsymbol{u}}}_{i}(t) ; \boldsymbol{\eta}^{h}(\mathbf{x})=\sum_{i=1}^{n_{\text {node }}} N_{i}(\mathbf{x}) \boldsymbol{\eta}_{i} \\
\dot{\overline{\boldsymbol{\varepsilon}}}^{h}(\mathbf{x}, t)=\sum_{e=1}^{n_{\text {elem }}} \phi^{(e)}(\mathbf{x}) \dot{\overline{\boldsymbol{\varepsilon}}}^{(e)}(t) \quad ; \quad \boldsymbol{\mu}^{h}(\mathbf{x})=\sum_{e=1}^{n_{\text {elem }}} \phi^{(e)}(\mathbf{x}) \boldsymbol{\mu}^{(e)} \\
\llbracket \dot{\boldsymbol{u}} \rrbracket^{h}(\mathbf{x}, t)=\sum_{e=1}^{n_{\text {elem }}} \phi^{(e)}(\mathbf{x}) \llbracket \dot{\boldsymbol{u}} \rrbracket^{(e)}(t) \quad ; \quad \boldsymbol{\beta}^{h}(\mathbf{x})=\sum_{e=1}^{n_{\text {elem }}} \phi^{(e)}(\mathbf{x}) \boldsymbol{\beta}^{(e)}
\end{gathered}
$$

where $n_{\text {elem }}$ and $n_{\text {node }}$ are, respectively, the number of nodes and elements of the finite element mesh, $\dot{\hat{\boldsymbol{u}}}_{i}$ is the nodal generalized displacement vector associated to the node $i, N_{i}$ are the standard isoparametric shape functions, $\llbracket \dot{u} \rrbracket^{(e)}$ and $\dot{\bar{\varepsilon}}^{(e)}$ are, respectively, the elemental displacement jump and regular strains degrees of freedom associated to the element $(e)$, and $\phi^{(e)}$ is the elemental collocation function defined in equation (23). 
The discretized versions of the total displacement field in equation (42), at global, $\dot{u}^{h}$, and elemental, $\dot{\boldsymbol{u}}^{(e)}$, levels read (see Figure 22):

$$
\begin{aligned}
\dot{\boldsymbol{u}}^{h}(\mathbf{x}, t)=\underbrace{\sum_{i=1}^{n_{\text {mode }}} N_{i}(\mathbf{x}) \dot{\hat{\boldsymbol{u}}}_{i}(t)}_{\dot{\hat{\boldsymbol{u}}}^{h}}+\sum_{e=1}^{n_{\text {elem }}} \underbrace{\left(\mathcal{H}_{\mathcal{S}}-\varphi^{h}\right)}_{\mathcal{M}_{\mathcal{S}}^{(h)}} \llbracket \dot{u} \rrbracket^{h} & \forall \mathbf{x} \in \mathcal{B} \\
\dot{\boldsymbol{u}}^{(e)}(\mathbf{x}, t) & =\underbrace{\sum_{i=1}^{4} N_{i}^{(e)}(\mathbf{x}) \dot{\hat{\boldsymbol{u}}}_{i}(t)}_{\dot{\hat{\boldsymbol{u}}}^{(e)}(\mathbf{x}, t)}+\underbrace{\left(\mathcal{H}_{\mathcal{S}}^{(e)}-\varphi^{(e)}\right)}_{\mathcal{M}_{\mathcal{S}}^{(e)}} \llbracket \dot{\boldsymbol{u}} \rrbracket^{(e)}(\mathbf{x}, t) \quad \forall \mathbf{x} \in \mathcal{B}^{(e)} \quad e=1, \ldots, n_{\text {elem }}
\end{aligned}
$$

and the indicatrix-function, $\varphi^{h}$, appearing in equation (57) is determined as follows:

$$
\begin{aligned}
\varphi^{h}(\mathbf{x}) & =\sum_{i=1}^{n_{\text {node }}} N_{i}(\mathbf{x}) \varphi_{i}=\sum_{i=1}^{n_{\text {node }}^{+}} N_{i^{+}}(\mathbf{x}) \\
\varphi^{(e)}(\mathbf{x}) & =\sum_{i=1}^{4} N_{i}^{(e)}(\mathbf{x}) \varphi_{i}^{(e)}=\sum_{i=1}^{4} N_{i^{+}}^{(e)}(\mathbf{x})
\end{aligned}
$$

where $\varphi_{i}$ is a nodal value, determined by the position of the crack-path-field, $\mathcal{S}^{h}$. Therefore, the indicatrix-function takes unit and null values for nodes belonging to $\mathcal{B}^{+}$and $\mathcal{B}^{-}$, respectively ${ }^{14}$. The subscript + refers to nodes belonging to $\mathcal{B}^{+}\left(\varphi_{i^{+}}=1\right)$ (see Figure 21 and Figure 22).
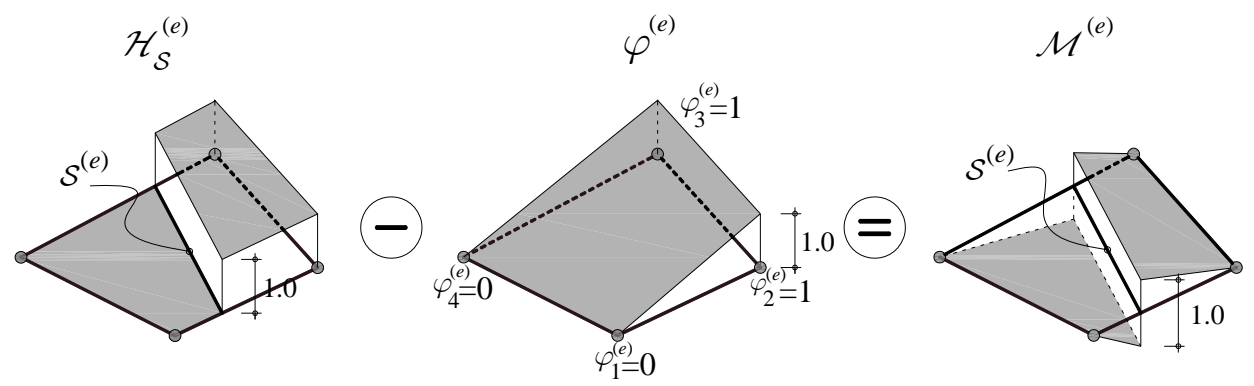

Figure 22. Elemental functions: Heaviside (step) function, $\mathcal{H}_{\mathcal{S}}^{(e)}$, indicatrix function, $\varphi^{(e)}$, and unit jump function, $\mathcal{M}^{(e)}$.

REMARK 4.2-1. As explained above, in order to construct the elemental indicatrix function $\varphi^{(e)}$, distinction between nodes belonging to $\mathcal{B}^{(e),+}$ and $\mathcal{B}^{(e),-}$ is necessary, and, therefore, information about the position of the discontinuity path inside the finite element is required. In addition, in order to ensure the continuity of function $\varphi$ in equation (43), the crack path has to be continuous across the sides of contiguous elements. Fulfilling this condition generally requires the use of cumbersome and code invasive techniques (e.g. crack-path-tracking algorithms or level set methods (Stolarska, Chopp et al. 2001; Oliver, Huespe et al. 2004)) to determine the precise position of $\mathcal{S}^{h}$ in the finite element mesh. The new crack-path-field technique, presented in section 2, becomes a powerful technique to achieve such a purpose. This issue will be tackled in subsequent sections.

${ }^{14}$ Therefore, its value $\varphi_{i} \in\{0,1\}$ at a given node indicates at what side of the discontinuity path the node lies, which justifies its name. 


\subsubsection{Strong-discontinuity regularization: finite element with embedded strong discontinuity.}

The spatial discretization of the CSDA variational problem in equations (46)-(47) requires some further elaboration.

At the element level, the unit vector $\mathbf{n}^{(e)}$, is assumed constant and normal to that part of the discontinuity path, $\mathcal{S}^{(e)}$, crossing the finite element (as a straight segment $\mathcal{S}^{(e)} \equiv \mathcal{B}^{(e)} \cap \mathcal{S}^{h}$ ) (see Figure 21-(b)). In addition, the generalized functions Dirac's delta, $\delta_{\mathcal{S}}$, and dipole-like, $\chi_{\mathcal{S}}^{+}$, (in equations (47) and (48)) are approximated, through $k$-regularized sequences, as follows (see Figure 23):
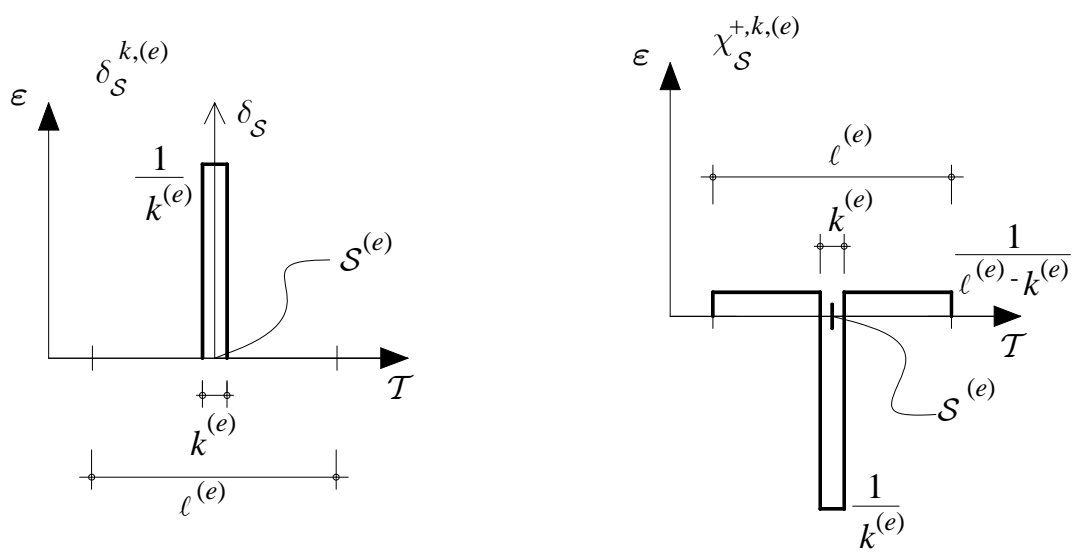

Figure 23. Dirac's delta, $\delta_{\mathcal{S}}$, and dipole-like, $\chi_{\mathcal{S}}^{+}$generalized functions approximated, through $k$-regularized sequences.

$$
\begin{aligned}
& \delta_{\mathcal{S}} \simeq \delta_{\mathcal{S}}^{k,(e)}(\mathbf{x})= \begin{cases}\frac{1}{k^{(e)}} & \forall \mathbf{x} \in \mathcal{B}^{k,(e)} \\
0 & \forall \mathbf{x} \in \mathcal{B}^{(e)} \backslash \mathcal{B}^{k,(e)}\end{cases} \\
& \chi_{\mathcal{S}}^{+} \simeq \chi_{\mathcal{S}}^{+, k,(e)}(\mathbf{x})= \begin{cases}-\frac{1}{k^{(e)}} & \forall \mathbf{x} \in \mathcal{B}^{k,(e)} \\
\frac{1}{\ell^{(e)}-k^{(e)}} & \forall \mathbf{x} \in \mathcal{B}^{(e)} \backslash \mathcal{B}^{k,(e)}\end{cases}
\end{aligned}
$$

In equation $(59), \delta_{\mathcal{S}}^{k,(e)}$ is an elemental k-regularized sequence approximating the Dirac's delta-function $\delta_{\mathcal{S}}$ inside the element $(e)$ in terms of the regularization parameter $k^{(e)}, \mathcal{B}_{k}^{(e)}$ is a band, of width $k^{(e)}$, crossing the element $\mathcal{B}^{(e)}$ (see Figure 21-(b)), containing the elemental crack-path $\mathcal{S}^{(e)}$, and $\ell^{(e)}$ is the elemental effective bandwidth computed as ${ }^{15}$ :

$$
\ell^{(e)}=\frac{\operatorname{meas}\left(\mathcal{B}^{(e)}\right)}{\operatorname{meas}\left(\mathcal{S}^{(e)}\right)}=\mathcal{O}\left(h^{(e)}\right)
$$

The elemental regularization parameter, $k^{(e)}$, can be written as a fraction, $\xi$ (considered the same for all the elements of the mesh), of the elemental effective bandwidth, i.e.:

$$
k^{(e)}=\xi \ell^{(e)} \quad \xi \in(0,1]
$$

where $\xi$ can take, in principle, any value in the semi-open interval $(0,1]$, with the only limitation of remaining inside the bounds of interval in terms of the computer accuracy.

15 When the computation of meas $\left(S^{(e)}\right)$ is done assuming its placement through the element barycenter, equation (60) returns the so-called consistent characteristic length used in some strainlocalization formulations to provide mesh-orientation objective dissipation (Oliver 1989). 
It can be readily checked that, with the definitions in equation (59) to (61) in hand, replacement of the regularized versions $\delta_{\mathcal{S}}^{k,(e)}$ and of $\chi_{\mathcal{S}}^{+, k,(e)}$ into the discrete version of the variational problem of equations (46) and (47) converge to the continuous problem as $h \rightarrow 0$. In addition, the softening k-regularized versions of the constitutive models ensure the proper dissipation of he fracture energy (Oliver and Huespe 2004a; Oliver and Huespe 2004b).

REMARK 4.2-2

It is interesting to notice the effects of the regularization procedure in the numerical integration rule involved in the numerical problem. From equations (59) and Figure 23 we realize that the regularized versions of $\delta_{\mathcal{S}}$ and $\chi_{\mathcal{S}}^{+}$are discontinuous inside the element. Therefore, a specific numerical integration rule accounting for this discontinuity has to be implemented in the spatial discrete version of equations (47). In the context of the adopted, element-wise-constant, approximation of the regular strains, $\bar{\varepsilon}^{h}$ and displacement jumps, $\llbracket \dot{u} \rrbracket^{h}$, (see equations (55) and (56)) this suggests consideration of two specific additional sampling points in every element capturing the discontinuity:

- One singular sampling point, named $\mathbf{x}_{\mathcal{S}}$, attached to that portion of the element where the discontinuity is regularized (the regularization band $\mathcal{B}^{k,(e)}$, in equations (59).

- One regular sampling point, named $\mathbf{x}_{\mathcal{B} \backslash \mathcal{S}}$, corresponding to the rest of the element $\left(\mathcal{B}^{(e)} \backslash \mathcal{B}^{k,(e)}\right.$ in equations (59).

The weight associated to the integration point differs in each case, as it is indicated in Table 4.2.1-1 in terms of the values of the regularized Dirac'sdelta, $\delta_{\mathcal{S}}, \chi_{\mathcal{S}}^{+}$and dipole-like generalized functions, and the corresponding areas of support.

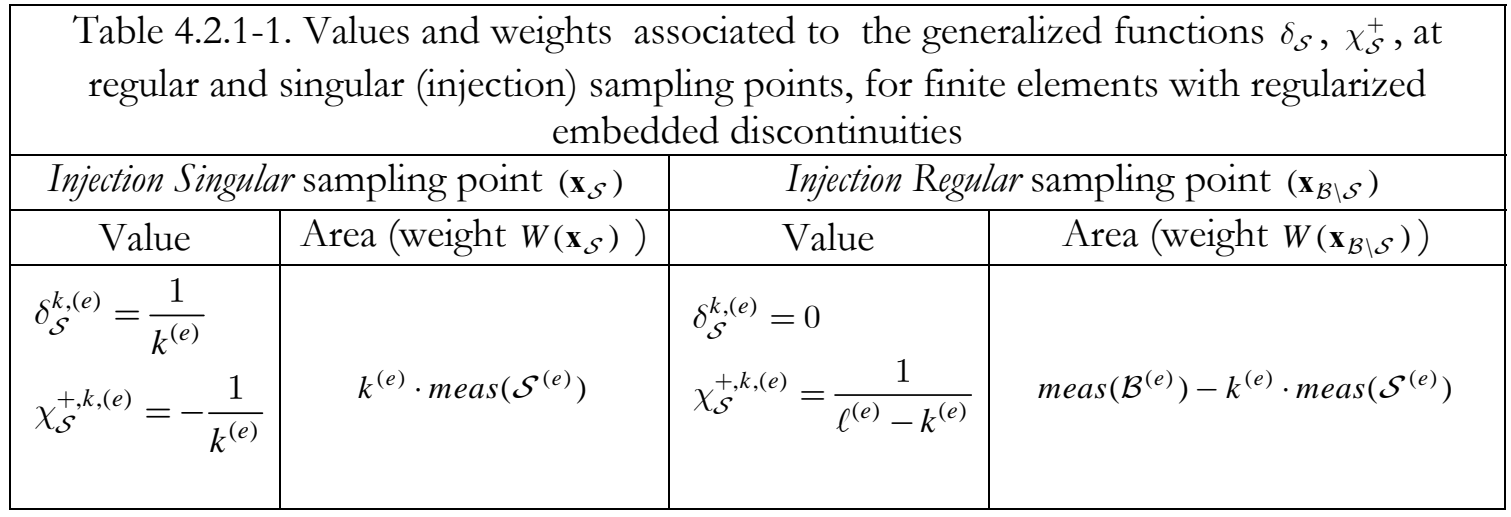

\subsubsection{Space-discretized mechanical problem}

Considering the spatial discretized version of the three field mixed formulation of section 4.1.3, and denoting the strong discontinuity injection domain as $\mathcal{B}_{\text {inj }} \subset \mathcal{B}$ (the set of elements to be injected), substitution of equations (54) to (56) into the variational problem in equations (47) yields 


$$
\begin{aligned}
& \left.\int_{\mathcal{B} \backslash \mathcal{B}_{i n j}} \nabla^{s} \boldsymbol{\eta}^{h}: \dot{\boldsymbol{\Sigma}}\left(\nabla^{S} \dot{\hat{\boldsymbol{u}}}^{h}\right) d \mathcal{B}+\sum_{\forall \mathcal{B}^{(e)} \in \mathcal{B}_{i n j}} \int_{\mathcal{B}^{(e)}} \nabla^{s} \boldsymbol{\eta}^{h}: \dot{\boldsymbol{\sigma}}^{(e)} d \mathcal{B}=W^{e x t}\left(\boldsymbol{\eta}^{h}, \dot{\mathbf{b}}, \dot{\mathbf{t}}^{*}\right) \quad \forall \boldsymbol{\eta}^{h} \in \hat{\mathcal{Y}}^{h} \quad a\right) \\
& \left.\int_{\mathcal{B}^{(e)}} \boldsymbol{\mu}^{(e)}:[\dot{\bar{\varepsilon}}^{(e)}-\underbrace{\left(\nabla^{S} \dot{\hat{\boldsymbol{u}}}^{h}-\left(\nabla \varphi^{h} \otimes \llbracket \dot{\boldsymbol{u}} \rrbracket^{(e)}\right)^{S}\right.}_{\dot{\hat{\varepsilon}}_{t}^{(e)}}] d \mathcal{B}=0 \quad \forall \mathcal{B}^{(e)} \in \mathcal{B}_{i n j} \quad \forall \boldsymbol{\mu}^{(e)} \quad b\right) \\
& \left\{\begin{array}{l}
\left.\int_{\mathcal{B}^{(e)}} \chi_{\mathcal{S}}^{+, k,(e)}\left(\boldsymbol{\beta}^{(e)} \otimes \mathbf{n}^{(e)}\right)^{S}: \dot{\boldsymbol{\sigma}}_{t} d \mathcal{B}=\boldsymbol{\beta}^{(e)} \cdot \int_{\mathcal{S}^{(e)}} \llbracket \dot{\boldsymbol{\sigma}} \rrbracket_{\mathcal{S}}^{+} d \mathcal{S} \cdot \mathbf{n}^{(e)}=0 \quad \forall \mathcal{B}^{(e)} \in \mathcal{B}_{\text {inj }} \quad c\right) \\
\Rightarrow\left[\llbracket \dot{\boldsymbol{\sigma}} \mathbb{\mathcal { S }}^{+,(e)} \cdot \mathbf{n}^{(e)}\right] \mathcal{S}^{(e)}=\mathbf{0}
\end{array}\right. \\
& \dot{\boldsymbol{\sigma}}^{(e)}=\dot{\boldsymbol{\Sigma}}\left(\dot{\bar{\varepsilon}}^{(e)}+\delta_{\mathcal{S}}^{k,(e)}\left(\llbracket \dot{\boldsymbol{u}} \rrbracket^{(e)} \otimes \mathbf{n}^{(e)}\right)^{S}\right)
\end{aligned}
$$

where $\delta_{\mathcal{S}}^{k,(e)}$ and $\chi_{\mathcal{S}}^{+, k,(e)}$ stand for the regularized Dirac's-delta or dipole-like functions in equations (59) (see Table 4.2.1-1).

Equation (62)-(b) can be trivially solved for the elemental strain values, $\dot{\bar{\varepsilon}}^{(e)}$, as ${ }^{16}$ :

$$
\dot{\bar{\varepsilon}}^{(e)}={\overline{\nabla^{S} \dot{\hat{\boldsymbol{u}}}^{(}}}^{(e)}-\left(\overline{\nabla \varphi^{h}}{ }^{(e)} \otimes \llbracket \dot{u} \rrbracket^{(e)}\right)^{S} \quad \forall \mathcal{B}^{(e)} \in \mathcal{B}_{i n j}
$$

where, again, the over-bar stands for average elemental values. Also, equation (62)-(c) can be solved for every element of the injection domain, $\mathcal{B}_{i n j}$, leading to the element-wise $\mathcal{S}$ inner traction continuity (see equation (50)):

$$
\underbrace{\dot{\boldsymbol{\Sigma}}\left(\dot{\bar{\varepsilon}}^{(e)}+\delta_{\mathcal{S}}^{k(e)}\left(\mathbf{n}^{(e)} \otimes \llbracket \dot{\boldsymbol{u}} \rrbracket^{(e)}\right)^{\mathcal{S}}\right.}_{\dot{\boldsymbol{\sigma}}_{\mathcal{S}}^{(e)}} \cdot \mathbf{n}^{(e)}=\underbrace{\dot{\boldsymbol{\Sigma}}\left(\dot{\bar{\varepsilon}}^{(e)}\right)}_{\dot{\boldsymbol{\sigma}}_{\mathcal{B} \backslash \mathcal{S}}^{(e)}} \cdot \mathbf{n}^{(e)} \forall \mathcal{B}^{(e)} \in \mathcal{B}_{i n j}
$$

Replacement of equations (63) and (64) into equations (62) yields the set of equations:

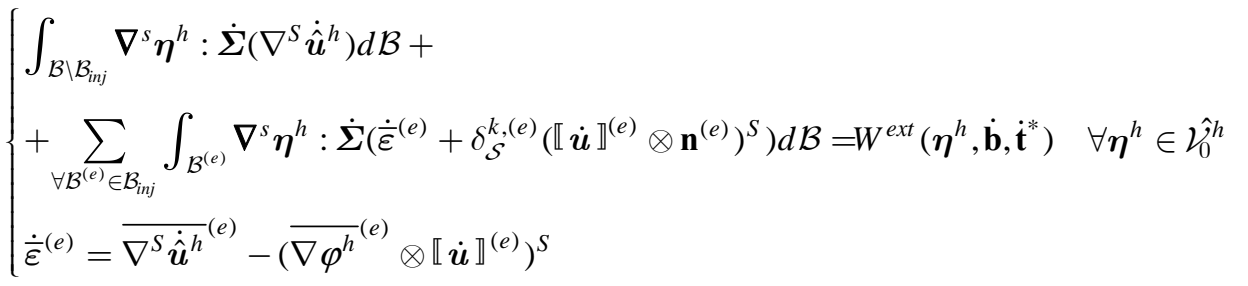

$$
\begin{aligned}
& \underbrace{\dot{\boldsymbol{\Sigma}}_{\mathcal{S}}\left(\dot{\bar{\varepsilon}}^{(e)}+\delta_{\mathcal{S}}^{k(e)}\left(\mathbf{n}^{(e)} \otimes \llbracket \dot{u} \rrbracket^{(e)}\right)^{S}\right.}_{\dot{\boldsymbol{\sigma}}_{\mathcal{S}}^{(e)}} \cdot \mathbf{n}^{(e)}=\underbrace{\dot{\boldsymbol{\Sigma}}_{\mathcal{B} \backslash \mathcal{S}}\left(\dot{\bar{\varepsilon}}^{(e)}\right)}_{\dot{\boldsymbol{\sigma}}_{\mathcal{B} \backslash \mathcal{S}}^{(e)}} \cdot \mathbf{n}^{(e)} \quad \forall \mathcal{B}^{(e)} \in \mathcal{B}_{i n j}
\end{aligned}
$$

Equation (65) is a set of two equations, in the unknowns $(\dot{\hat{\boldsymbol{u}}} ; \llbracket \dot{\boldsymbol{u}} \rrbracket)$, since the third field, $\dot{\bar{\varepsilon}}$, has been condensed. As usual in the CSDA, to prevent the trivial solution $\llbracket \dot{\boldsymbol{u}} \rrbracket^{(e)}=0$ elastic unloading is forced at $\mathcal{B} \backslash \mathcal{S}$ (Oliver and Huespe 2004a). In equation (65)-(b), $\dot{\boldsymbol{\Sigma}}_{\mathcal{B} \backslash \mathcal{S}}$ stands for the incremental stresses evaluated at $\mathcal{B} \backslash \mathcal{S}$ from an incrementally elastic constituve model and $\dot{\boldsymbol{\Sigma}}_{\mathcal{S}}$ stands for the incremental stresses evaluated at $\mathcal{S}$ from the chosen $\mathrm{k}$ regularized strain-softening continuum constitutive model).

REMARK 4.2-3. A significant property of the proposed mixed formulation, with respect to standard strong discontinuity formulations, is the release of the so-called stress locking, associated to the inability of some finite elements (typically bi-linear quadrilaterals equipped with four integration points) to capture the independent rigid-body motions at the two sides of an element crossed by a discontinuity. This inability translates into generation of spurious stresses, which give the name to the phenomenon. In appendix B this issue is deeper studied.

\footnotetext{
${ }^{16}$ It can be proven that the term $\left(\mathcal{M}_{\mathcal{S}} \otimes \nabla \llbracket \dot{\boldsymbol{u}} \rrbracket_{t}\right)^{S}$ in equation (47)-(b) can be neglected ((Dias 2012;
} Dias, Oliver et al. 2012) 
Large computational savings can be obtained if, in the variational problem in equation (65), the element displacement jump, $\llbracket \dot{u} \rrbracket^{(e)}$, is condensed out by solving independently the (non-linear) equation (65)-(b), for every element $\mathcal{B}^{(e)} \subset \mathcal{B}_{i n j}^{(e)}$, and replacing the obtained $\llbracket \dot{\boldsymbol{u}} \rrbracket^{(e)}=\mathbf{g}\left(\dot{\hat{\boldsymbol{u}}}^{h}\right)$ into equation 65)-(a).

This gives rise to the condensed variational mechanical problem in BOX 4.2-1.

BOX 4.2-1: Discontinuous-displacement mode (DDM) injection. Condensed problem

$$
\left\{\begin{array}{l}
\int_{\mathcal{B} \backslash \mathcal{B}_{i n j}} \nabla^{S} \boldsymbol{\eta}^{h}: \dot{\boldsymbol{\Sigma}}\left(\nabla^{S} \dot{\hat{\boldsymbol{u}}}^{h}\right) d \mathcal{B}+ \\
+\sum_{\forall \mathcal{B}^{(e)} \in \mathcal{B}_{i n j}} \int_{\mathcal{B}^{(e)}} \nabla^{S} \boldsymbol{\eta}^{h}: \dot{\boldsymbol{\Sigma}}\left(\dot{\overline{\boldsymbol{\varepsilon}}}^{(e)}+\delta_{\mathcal{S}}^{k(e)}\left(\mathbf{g}\left(\dot{\hat{\boldsymbol{u}}}^{h}\right) \otimes \mathbf{n}^{(e)}\right)^{S}\right) d \mathcal{B}=W^{e x t}\left(\boldsymbol{\eta}^{h}, \dot{\mathbf{b}}, \dot{\mathbf{t}}^{*}\right) \\
\dot{\overline{\boldsymbol{\varepsilon}}}^{(e)}={\overline{\nabla^{S} \dot{\hat{\boldsymbol{u}}}^{h}}}^{(e)}-\left({\bar{\nabla} \varphi^{h}}^{(e)} \otimes \mathbf{g}\left(\dot{\hat{\boldsymbol{u}}}^{h}\right)^{(e)}\right)^{S}
\end{array}\right.
$$

Details about this element-wise condensation procedure are given in appendix A.2

REMARK 4.2-4. In equation (66), $\mathbf{n}^{(e)}$ stands for the unit vector computed by resorting to the discontinuous material bifurcation analysis in equation (36) (as the bifurcation direction). However, the algorithms providing these vectors (Oliver and Huespe 2004a; Oliver, Huespe et al. 2010), usually furnish two admissible local directions. In applications, and in order to perform the DDM injection in BOX 4.2-1, it is necessary to select, among these local directions, the one more compatible with the global failure mechanism provided by the crack-path-field technique. This selection can be done with different criteria (see Appendix A.3 for details).

\subsubsection{Injection of a displacement discontinuity mode (DDM) in a two-stage procedure}

As commented above, the use of finite elements with embedded discontinuities, requires information about the placement of the elemental discontinuity path, $\mathcal{S}^{(e)}$ inside the element that is going to be injected a DDM (see Figure 21-(b) and

REMARK 4.2-1). Typically, global tracking-like algorithms (Oliver, Huespe et al. 2004) are required for such a purpose. Here an alternative of local character is proposed, based on the new crack-path-field technique in combination with the injection of specific strain fields at two different stages:

In a first stage (the tracing stage ${ }^{17}$ ), an element- wise constant strain mode (CSM) is injected, in an appropriated injection domain, based on the procedures and algorithms described in section 3.3. This first injection is devoted exclusively to provide reliable information about the propagation of a strain-localization field. In section 3.3, it has been shown that the improved flexibly stemming from the weak enforcement of the compatibility equation, endows the resulting element with remarkable good ability for the correct propagation of the strain localization in initial stages. Nevertheless, the element equipped with the CSM kinematics still exhibits a limited capability to match a real displacement discontinuity and some degree of stress locking appears. Those finite elements injected with a CSM in this stage supply the localized strain-like internal variable $\alpha^{h}(\mathbf{x}, t)$, identified as the strongly

\footnotetext{
${ }^{17}$ This name comes from the fact that the "strain localization procedure" used in the stage in combination with the crack propagation problem described in Section 2, allows tracing the position of the discontinuity (the crack propagation path). This information is essential to inject, subsequently, displacement discontinuity modes.
} 
localized field in the algorithm in BOX 2.2-1. Then, the outcomes of this algorithm, e.g. the crack-path-field $\mu_{t}$ and the crack-path-set $\Gamma_{t}^{h}$, are used to predict the discontinuity propagation path required in the second stage.

Therefore, in a second stage, as soon as the element is considered endowed with reliable information about the crack-path position (typically the crack-path-field $\mu_{t}$ and the crackpath-set $\Gamma_{t}$, see Figure 24) a discontinuous displacement mode is injected according with the procedure described in next sections.

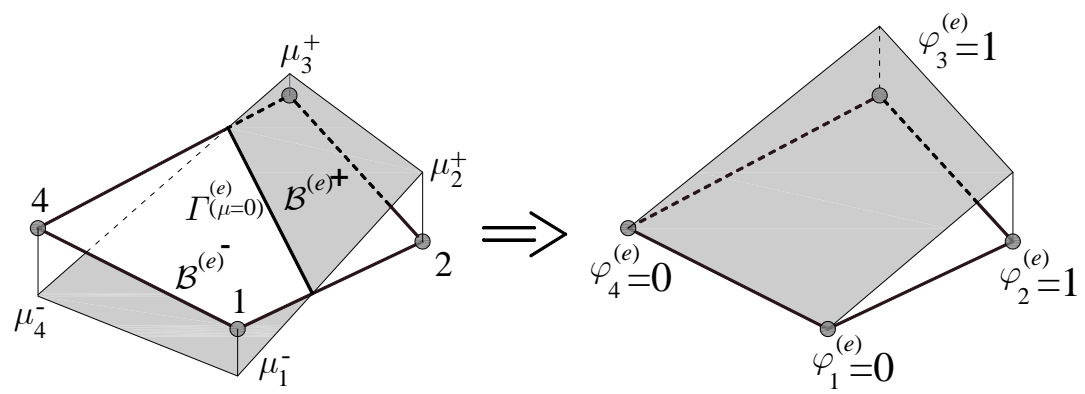

Figure 24. Construction of the nodal values of the indicatrix-function, $\varphi_{i}^{(e)}$, for injecting the strong discontinuity, in terms of the sign of the nodal values of the crack-path-field $\mu_{i}$.

\subsubsection{The injection domains}

The procedure can be inserted in the context of the multi-domain injection technique presented in section 3.2. (see equations (18) and (19) and Figure 25) for $n_{i n j}=2$, as follows:

$$
\begin{aligned}
& \mathcal{B}_{\text {inj }}(t)=\bigcup_{i=1}^{i=2} \mathcal{B}_{\text {inj }}^{(i)}(t)=\mathcal{B}_{\text {loc }}(t) \cup \mathcal{B}_{\text {dis }}(t) ; \\
& \mathcal{B}_{\text {loc }}(t) \cap \mathcal{B}_{\text {dis }}(t)=\varnothing
\end{aligned}
$$

where $\mathcal{B}_{\text {inj }}(t)$ is the (total) injected domain, at time $t$, and $\mathcal{B}_{\text {loc }}(t)$ and $\mathcal{B}_{\text {dis }}(t)$ are, respectively the sub-domains where the CSM and the DDM are being injected. They are defined as:

$$
\begin{aligned}
& \mathcal{B}_{\text {inj }}(t):=\left\{\bigcup_{e} \mathcal{B}^{(e)} ; \quad t \geq t_{B}\left(\mathbf{x}_{C}^{(e)}\right) ; \dot{\alpha}\left(\mathbf{x}_{C}^{(e)}, t\right)>0\right\} \\
& \mathcal{B}_{\text {dis }}(t):=\left\{\bigcup_{e} \mathcal{B}^{(e)} \in \mathcal{B}_{\text {inj }}(t) ; q\left(\mathbf{x}_{C}^{(e)}, t\right) \leq q_{\text {dis }}\left(\mathbf{x}_{C}^{(e)}\right)=\bar{\gamma} q_{b i f}\left(\mathbf{x}_{C}^{(e)}\right) ; \mathcal{B}^{(e)} \cap \Gamma_{t} \neq \varnothing\right\} \\
& \mathcal{B}_{\text {loc }}(t)=\mathcal{B}_{\text {inj }}(t) \backslash \mathcal{B}_{\text {dis }}(t)
\end{aligned}
$$

where $\mathbf{x}_{C}^{(e)}$ stands for the barycenter of the element " $e$ ".
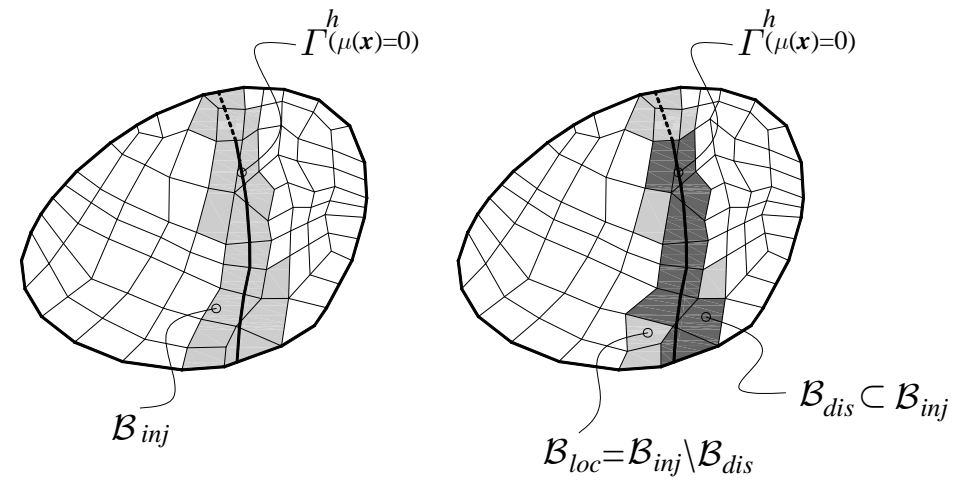

Figure 25. Localized constant strain mode (CSM) injection domain, $\mathcal{B}_{l o c}$, and discontinuous displacement mode (DDM) injection domain, $\mathcal{B}_{\text {dis }}$.

A more detailed description of the domains defined in equation (68) is given next: 
- $\mathcal{B}_{\text {inj }}(t)$ is the set of elements fulfilling the following conditions at their barycenter $\mathbf{x}_{C}^{(e)}$ :

- The discontinuous bifurcation condition, in equation (36) has been fulfilled in a previous time $\left(t \geq t_{B}\left(\mathbf{x}_{C}^{(e)}\right)\right)$; i.e.: the element is ready to accommodate strain localization.

- The element is in in-loading regime at the current time $\left(\dot{\alpha}\left(\mathbf{x}_{C}^{(e)}, t\right)>0\right)$ since unloading elements cannot localize.

- $\quad \mathcal{B}_{\text {dis }}(t)$ is the set of elements of $\mathcal{B}_{\text {inj }}(t)$, which fulfill the following conditions:

- The barycenter has achieved a "sufficient" degree of softening. The degree of softening is measured in terms of the stress-like internal variable (see Figure 26) defined through $\dot{q}=\mathcal{H} \dot{\alpha},\left.q\right|_{t=0}=q_{0}$, where $q_{0}$ (the parameter characterizing the elastic strength) and $\mathcal{H}<0$ (the softening modulus, related to the fracture energy) are material properties. Parameter $q_{b i f}\left(\mathbf{x}_{C}^{(e)}\right)$ is the value taken by the variable at the bifurcation time $t_{B}$. The "sufficient" degree character of the softening is imposed by the value $q_{d i s}\left(\mathbf{x}_{C}^{(e)}\right)=\bar{\gamma} q_{b i f}\left(\mathbf{x}_{C}^{(e)}\right)$ in terms of the parameter $\bar{\gamma}$. Typically $q_{\text {dis }}$ (smaller than $\left.q_{b i f}\right)$ is a value close to $q_{b i f}(\bar{\gamma} \in[0.8,1.0])$.

- They are crossed by the discontinuity path (through condition $\left.\mathcal{B}^{(e)} \cap \Gamma_{t} \neq \varnothing\right)$. This identification is done in terms of the nodal values of the crack path field, $\mu(\mathbf{x}, t)$, being of different sign (see Figure 27).

Elements belonging to $\mathcal{B}_{\text {dis }}(t)$ are incrementally injected a discontinuous displacement mode (DDM).

- $\mathcal{B}_{\text {loc }}(t)$ is the remaining set of elements of $\mathcal{B}_{\text {inj }}(t)$ not belonging to $\mathcal{B}_{\text {dis }}(t)$. They are injected a constant localization mode (CSM).

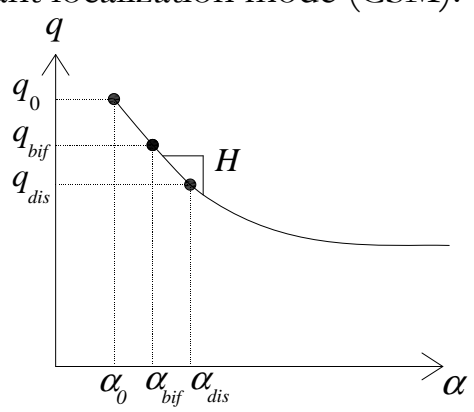

Figure 26. Thresholds in the evolution of the stress/strain-like internal variables.
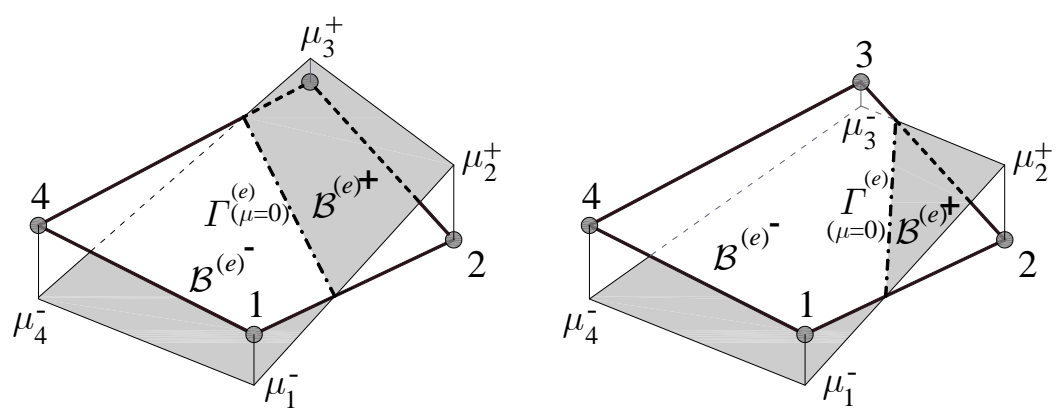

Figure 27 Elemental crack-propagation field $\mu^{(e)}$ in elements belonging to $\mathcal{B}_{\text {dis }}$. Change of sign of the nodal values $\mu_{i}$ along a side indicates that the elemental discontinuity path $\Gamma^{(e)}$ crosses that side. 
REMARK 4.2-5. In general, at the neighborhood of the crack tip, where material points are near to fulfill the bifurcation condition, yielding and damage still occur in a diffuse manner. In order to inject successfully the DDM strain mode, it is important to obtain in advance (from the crackpath field technique) reliable information about the propagation of the crack path. This is why, in the definition of the domain, $\mathcal{B}_{\text {dis }}(t)$, in equation (68)-(b), it is required that the finite element (sampled at its barycenter) must achieve a "sufficient" degree of softening, which is controlled directly by the parameter $\bar{\gamma}$. From the author's experience, $\bar{\gamma}=0.95$ provides a good balance between the (little) error produced by the delay in the injection of the DDM after bifurcation and the necessity of having reliable information on the crack path prior to that injection. Nevertheless, for some very specific cases other values of $\bar{\gamma}$ can be more effective (see REMARK 5.1-1 and REMARK 5.5-1).

\subsubsection{Stress sampling and numerical quadrature rule}

The injection procedure described in this section is aimed at being effectively implemented in a finite element code, on the basis of an underlying "standard element" (in this case the four-noded quadrilateral element) in a fairly simple manner and with little code invasion. For this purpose, the following effective-stress-based integration scheme is proposed.

The standard (four) sampling/integration (Gauss) points are complemented with two additional sampling points (placed at the center of the element ${ }^{18}$ ), see Figure 28. They are termed injection sampling points and denoted $\mathbf{x}_{\mathcal{B} \backslash \mathcal{S}}$ (the regular injection point) and $\mathbf{x}_{\mathcal{S}}$ (the singular injection point).

Some entities (typically the stresses) are then additionally sampled, and stored, at these injection-sampling points as it is done in the regular sampling points. The following stress fields are then considered at the discrete time $t_{n+1}$ :

- Regular (physical) stresses $\sigma_{n+1}^{(e)}\left(\mathbf{x}_{i}\right)$ : computed at all sampling points $\left(\mathbf{x}_{i}, i=1,2,3,4, \mathcal{B} \backslash \mathcal{S}, \mathcal{S}\right)$ in terms of the corresponding compatible strains $\nabla^{S} \boldsymbol{u}_{n+1}^{h}{ }^{(e)}\left(\mathbf{x}_{i}\right)$.

- Effective stresses $\tilde{\boldsymbol{\sigma}}_{n+1}^{(e)}\left(\mathbf{x}_{i}\right)$ : appropriated combinations of the regular stresses at the sampling points to return equivalent stresses for numerical integration purposes. They are only computed and stored at the standard (Gauss) sampling points $\left(\mathbf{x}_{i}, i=1,2,3,4\right)$.

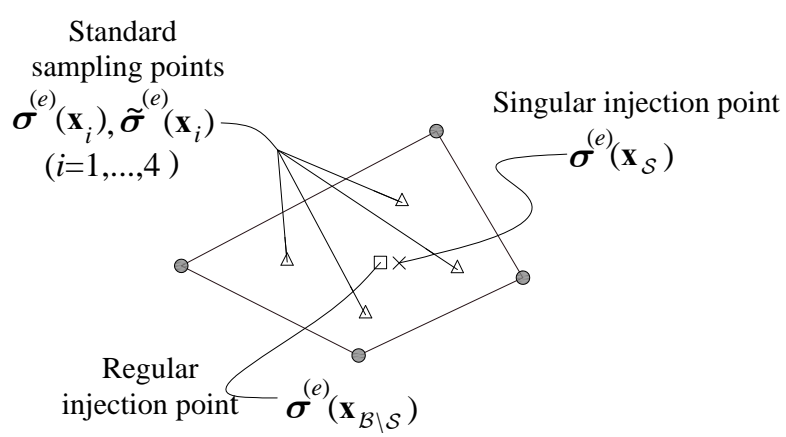

Figure 28 Sampling points involved in the numerical integration procedure.

\footnotetext{
${ }^{18}$ therefore, sampling of elemental continuous functions at those points provide approximate values of the elemental averages of those functions (see REMARK 3.3-1).
} 


\subsubsection{Time integration of the mechanical residue for the general strain injection case.}

Let us consider the general strain injection case, when the body $\mathcal{B}$ is split, at time $t$, into the disjoint parts $\mathcal{B}_{\text {inj }}(t)$ and $\mathcal{B} \backslash \mathcal{B}_{\text {inj }}(t)$ where, in turn, $\mathcal{B}_{\text {inj }}(t)=\mathcal{B}_{\text {loc }}(t) \cup \mathcal{B}_{\text {dis }}(t)$. Let us, consequently, consider a constant-strain mode (CSM) injection in $\mathcal{B}_{\text {loc }}(t)$ combined with a mixed stabilized formulation in $\mathcal{B} \backslash \mathcal{B}_{\text {inj }}(t)$, according with section 3.3 .4 (summarized in BOX 3.3-2) and a discontinuous-displacement-mode (DDM) injection in $\mathcal{B}_{\text {dis }}(t)$, as explained in section 4.2.2 (summarized in BOX 4.2-1), see Figure 29.
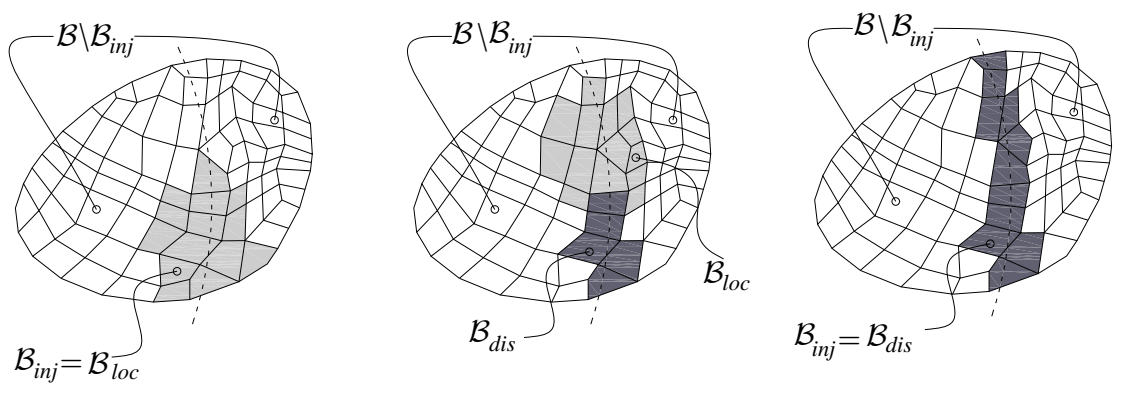

Figure 29 Evolution of the injection domains for three typical stages of loading

As commented in section 4.2.4.1, the combination of CSM injection and a DDM injection requires, in principle specific integration rules, i.e.: a standard four-point Gauss quadrature rule, $\mathbf{x}_{i}, i=(1,2,3,4)$, in $\mathcal{B} \backslash \mathcal{B}_{\text {inj }}(t)$, the integration with a single integration point, at the barycenter of the element, $\mathbf{x}_{C} \equiv \mathbf{x}_{\mathcal{S}}$, in $\mathcal{B}_{l o c}(t)$, and a two-point integration rule, $\mathbf{x}_{\mathcal{B} \backslash \mathcal{S}}$ and $\mathbf{x}_{\mathcal{S}}$ (the injection points in Table 4.2.1-1) in $\mathcal{B}_{\text {dis }}(t)$. This domain-specific integration rule can become cumbersome in two senses:

1) Domains $\mathcal{B} \backslash \mathcal{B}_{\text {inj }}(t), \mathcal{B}_{\text {loc }}(t)$ and $\mathcal{B}_{\text {dis }}(t)$ change with time. This poses, in principle, some additional problems on the time integration of the resulting rate of mechanical balance of forces, $\dot{\boldsymbol{R}}_{\text {mech }}\left(\dot{\hat{\mathbf{u}}}_{n+1}^{h}(t), t\right)$.

2) The implementation of those specific integration rules in a standard finite element code, which becomes code-invasive.

However, by resorting to the definition of some ad-hoc stress entities (the so called effective stresses, $\left.\tilde{\boldsymbol{\sigma}}^{(e)}\left(\mathbf{x}_{i}, t\right)(i=1,2,3,4)\right)$, as appropriate combinations, at the standard Gauss points, of the physical stresses at all sampling points, the integration rule can be simplified to a more appropriated four-points integration rule, common at all points in the domain $\mathcal{B}$. Appendix A.1 is devoted to explain and justify this issue.

The final, time-integrated, mechanical residue reads,

$$
\boldsymbol{R}_{\text {mech }}\left(\tilde{\boldsymbol{\sigma}}_{t}\left(\hat{\boldsymbol{u}}_{n+1}^{h}\right)\right)=\sum_{\forall \mathcal{B}^{(e)} \subset \mathcal{B}} \int_{\mathcal{B}^{(e)}} \mathbf{B}^{(e)^{T}}(\mathbf{x}) \cdot\left\{\tilde{\boldsymbol{\sigma}}_{n+1}^{(e)}(\mathbf{x})\right\} d \mathcal{B}-\mathbf{F}_{n+1}^{e x t}=\mathbf{0}
$$

where the effective stresses $\tilde{\boldsymbol{\sigma}}_{n+1}^{(e)}\left(\mathbf{x}_{i}\right)$ are derived in Appendix A.1 and displayed in BOX 4.2-2.

In equation (69), $\mathbf{F}_{n+1}^{e x t}\left(\mathbf{b}, \mathbf{t}^{*}\right)$ stands for the external forces, computed in a regular manner in terms of the body forces, $\mathbf{b}$, and the boundary tractions, $\mathbf{t}^{*}, \mathbf{B}^{(e)}(\mathbf{x})$ is the standard elemental deformation matrix for the four-node quadrilateral element and bracketed notation $\{(\bullet)\}$ is used to denote the vector (Voigt's notation) form of the symmetric second order entity $(\bullet)$.

REMARK 4.2-6. It is worth noting that the strain injection procedures proposed in this work, crucially rely on the incremental character of the injected strains, this allowing the smooth evolution, along time, of the corresponding strains and 
stresses, consistently with the evolutionary character of the injection domains. This is the reason for the B.V.P being initially stated in rate form in equations (45) and for the resulting rate form of the mechanical residue (stemming from the variational problem in equations (47)) to be integrated in time, according with the procedure presented in Appendix 1 and yielding equations (69).

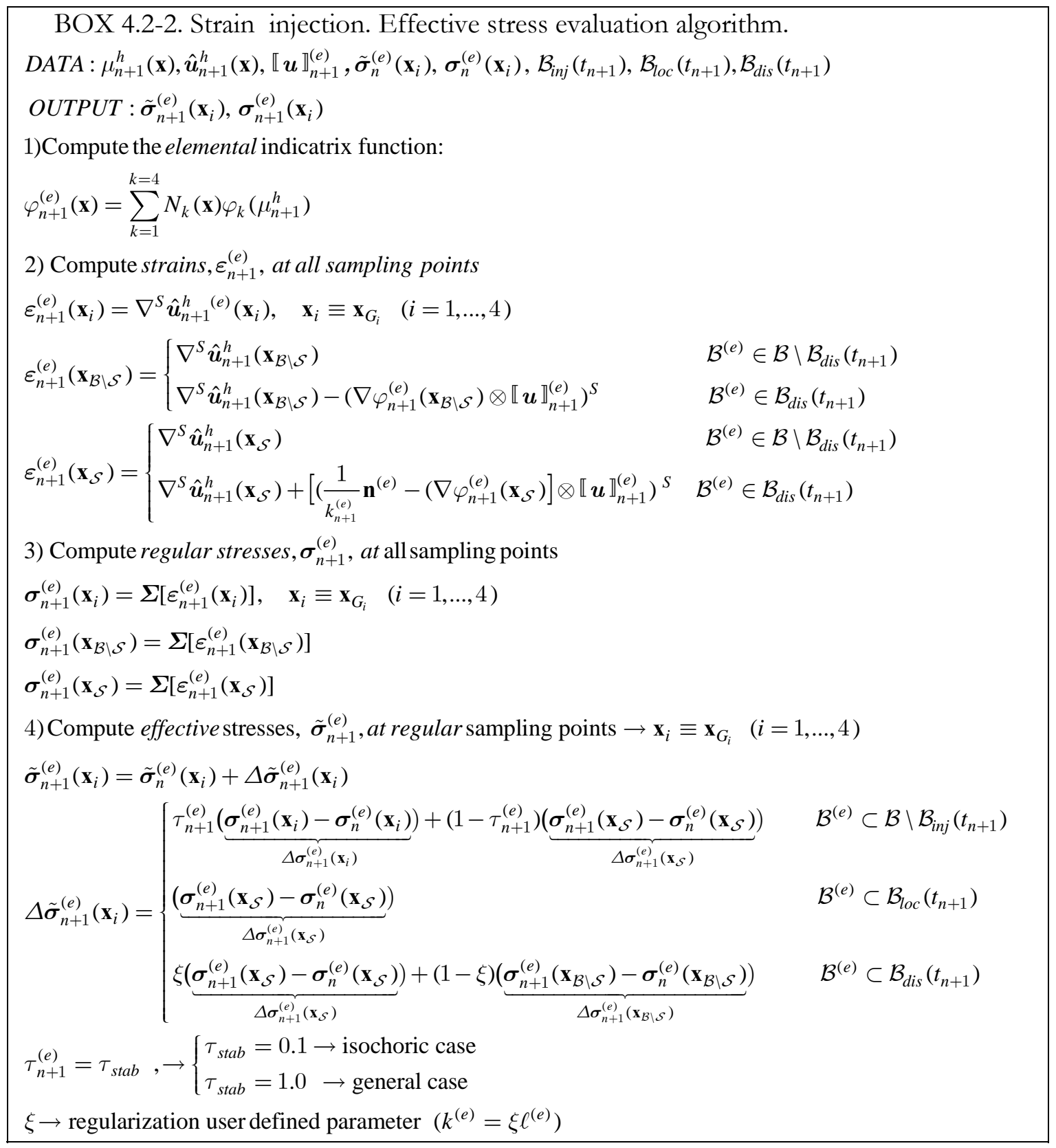

REMARK 4.2-7. For implementation purposes, determination of belonging of a given element $\mathcal{B}^{(e)}$ to the different injection domains, $\mathcal{B} \backslash \mathcal{B}_{i n j}\left(t_{n+1}\right), \mathcal{B}_{l o c}\left(t_{n+1}\right)$ and $\mathcal{B}_{\text {dis }}\left(t_{n+1}\right)$, as requested in BOX 4.2-2 is systematically stated by defining an injection status indicator, (IS $)_{n+1}^{(e)} \in\{0,1,2\}$ In appendix A.5 the corresponding definitions and a systematic procedure for determination of this indicator are presented. 


\subsection{Staggered resolution of the coupled propagation-injection problem}

The crack-path-field problem defined in BOX 2.2-1 is stated in terms of $\alpha^{h}$ (the localized strain-like internal variable, which depends directly in the solution of the nonlinear mechanical problem in equation (69). On the other hand this mechanical problem also depends on the crack path, $\mu^{h}$, obtained from that crack path field problem in BOX 2.2-1.

Thus, both problems are coupled, and two sets of discretized, in time and space, equations can be written in terms of the corresponding residuals:

$$
\begin{array}{ll}
\boldsymbol{R}_{\text {mech. }}\left(\hat{\boldsymbol{u}}_{n+1}^{h}, \mu_{n+1}^{h}\right)=\mathbf{0}, & (\text { a }) \\
\boldsymbol{R}_{\text {prop }}\left(\hat{\boldsymbol{u}}_{n+1}^{h}, \mu_{n+1}^{h}\right)=\mathbf{0}, & (b)
\end{array}
$$

where $\boldsymbol{R}_{\text {mech. }}=\underset{\mathcal{B}^{(e)} \in \mathcal{B}}{\mathcal{A}}\left(\boldsymbol{R}_{\text {mech }}^{(e)}\right)$, stands for the residual of the non-linear mechanical problem, in equation (69) assembled from the residuals of the individual elements $\boldsymbol{R}_{\text {mech }}^{(e)}$. Expressions for computing $\boldsymbol{R}_{\text {mech }}^{(e)}$ are provided in the Appendix A.2. In equations (67)-(b) $\boldsymbol{R}_{\text {prop }}$ stands for the residual of crack propagation problem ${ }^{19}$ in BOX 2.2-1.

Thinking of the strain injection procedure, it can be noticed that in the initial stages of the non-linear loading process $\mathcal{B}_{\text {dis }}=\varnothing$, no discontinuity is injected and therefore the problem in equation (70) is uncoupled in the sense propagation-problem $\rightarrow$ mechanical-problem. In this case equation (71)-(a) can be first directly solved for $\hat{u}_{n+1}^{h}$ and then, equation (71)-(b) can be solved for $\mu_{n+1}^{h}$ :

$$
\begin{aligned}
& \boldsymbol{R}_{\text {mech. }}\left(\boldsymbol{u}_{n+1}^{h}\right)=\mathbf{0} \rightarrow \boldsymbol{u}_{n+1}^{h}, \\
& \boldsymbol{R}_{\text {prop }}\left(\boldsymbol{u}_{n+1}^{h}, \mu_{n+1}^{h}\right)=\boldsymbol{R}_{\text {prop }}^{*}\left(\mu_{n+1}^{h}\right)=\mathbf{0} \rightarrow \mu_{n+1}^{h} .
\end{aligned}
$$

As soon as at least one element belongs to $\mathcal{B}_{\text {dis }}$, the problem becomes coupled in both senses.

However, numerical experiences show that the coupling is weak. In fact, the dependence of the mechanical problem on the variable $\mu^{h}(\mathbf{x})$ is only through the instantaneous position of the discontinuity path $\mathcal{S}^{h}$, to determine the indicatrix function $\varphi$ and the sets $\mathcal{B}_{\text {dis }}$ in BOX 4.2-2, so that the coupling between $\boldsymbol{u}_{n+1}^{h}$ and $\mu_{n+1}^{h}$ in the term $\boldsymbol{R}_{\text {mech }}\left(\boldsymbol{u}_{n+1}^{h}, \mu_{n+1}^{h}\right)$ can be considered weak. This suggests the use of a staggered simplified procedure, replacing $\mu_{n+1}^{h}$ by $\mu_{n}^{h}$ in equation (70)-(a), this leading to the set of uncoupled equations

$$
\begin{aligned}
& \boldsymbol{R}_{\text {mech }}\left(\boldsymbol{u}_{n+1}^{h}, \mu_{n}^{h}\right)=\boldsymbol{R}_{\text {mech }}^{*}\left(\boldsymbol{u}_{n+1}^{h}\right)=\mathbf{0} \rightarrow \boldsymbol{u}_{n+1}^{h} \\
& \boldsymbol{R}_{\text {prop }}\left(\boldsymbol{u}_{n+1}^{h}, \mu_{n+1}^{h}\right)=\boldsymbol{R}_{\text {prop }}^{*}\left(\mu_{n+1}^{h}\right)=\mathbf{0} \rightarrow \mu_{n+1}^{h}
\end{aligned}
$$

REMARK 4.3-1.The staggered resolution of the coupled problem that leads to the uncoupled equations (72) allow envisaging the crack-path-field problem in BOX 2.2-1 as a post-processing procedure (typically a double smoothing) of the strain-like internal variable $\alpha^{h}(\mathbf{x}, t)$ in the mechanical problem. The crack propagation problem can be then interpreted as a local (element wise based) tracking algorithm that can be straightforwardly, implemented in a finite element code in a non-invasive manner.

\footnotetext{
${ }^{19}$ which, unlike the mechanical problem, can be proven to be a linear problem.
} 
Specific details about the staggered finite element implementation of the discontinuous displacement mode (DDM) injection techniques and the coupled crack-path-field can be found in Appendices A.2 and A.3, respectively.

\section{Representative numerical simulations}

A set of representative benchmarks have been selected to assess the proposed combined crack-path-field and strain injection techniques. Specific aspects to be assessed are:

1) The ability of the proposed methodology to propagate correctly the captured crack, disregards the type and orientation of the finite element mesh.

2) Its ability to improve the results provided by the strain-localization based injection methodology, explored in section 3.3, getting it rid of the stress-locking effects.

3) The ability of the proposed methods to provide less dissipative, and, therefore, more physically meaningful numerical solutions, for the structural response of problems displaying propagating material failure.

For comparison purposes, reference analytical solutions are retrieved (when available) in addition to computational solutions based on classical strain-localization methods, either standard irreducible elements or the improved B-Bar mixed approaches (Hughes 1980).

\subsection{Strip in homogeneous uniaxial tensile stress state.}

The problem in section 3.3.6 is retaken again in the context of the DDM injection techniques, and its available theoretical solution is used for quantitative assessment of the results. Figure 30 shows the geometrical and mechanical properties of the problem.

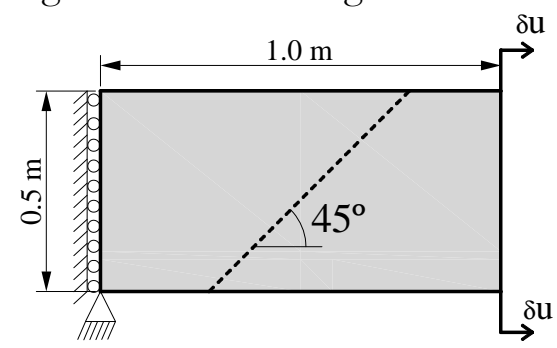

\begin{tabular}{cccc:c}
\hline $\begin{array}{c}\sigma_{y} \\
{[k P a]}\end{array}$ & $\begin{array}{c}E \\
{[k P a]}\end{array}$ & $v$ & $G_{f}$ & $\bar{\gamma}$ \\
\hline 1,0 & 120 & 0,49 & 22.7 & 0.99 \\
\hline
\end{tabular}

Figure 30. Strip subjected to tensile stretching using a $J_{2}$ plasticity model.

Geometrical data and considered material properties.

Two different challenging meshes are selected to check the performance of the method. One of them is unstructured (see Figure 31-(a)), with irregular mesh size, and the second one (see Figure 31-(b)) is misaligned, i.e. the mesh lines are oriented with an inclination that drifts from the theoretical slip-line inclination: $65^{\circ}$ and $45^{\circ}$, respectively. This forces the propagation algorithm to break the "natural" trend of localized solutions to follow the mesh alignment (mesh-bias dependence(Rots 1988)). Parameter $\bar{\gamma}$, establishing the threshold for injection of the DDM after bifurcation (see equation (68)-(b) and Figure $26)$ is taken $\bar{\gamma}=0.99$. For the regularization parameter in equation (61) it was used $k^{(e)}=\ell^{(e)}(\xi=1.0)$ although very similar results are obtained by using different values of $\xi=\in(0,1]$. 
(a) Mesh i

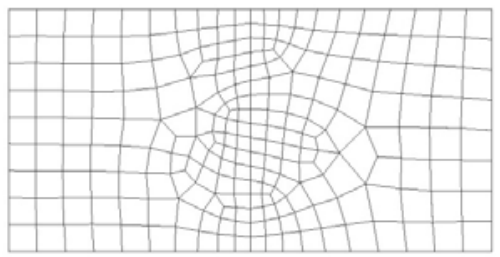

(b) Mesh ii

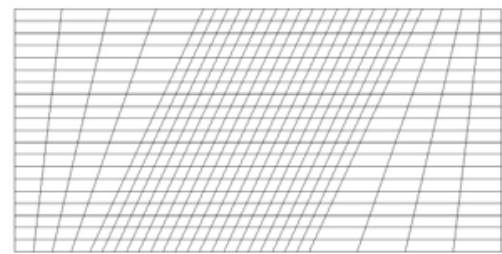

Figure 31. Strip subjected to tensile stretching. Finite element meshes:

a) unstructured mesh, b) misaligned mesh.

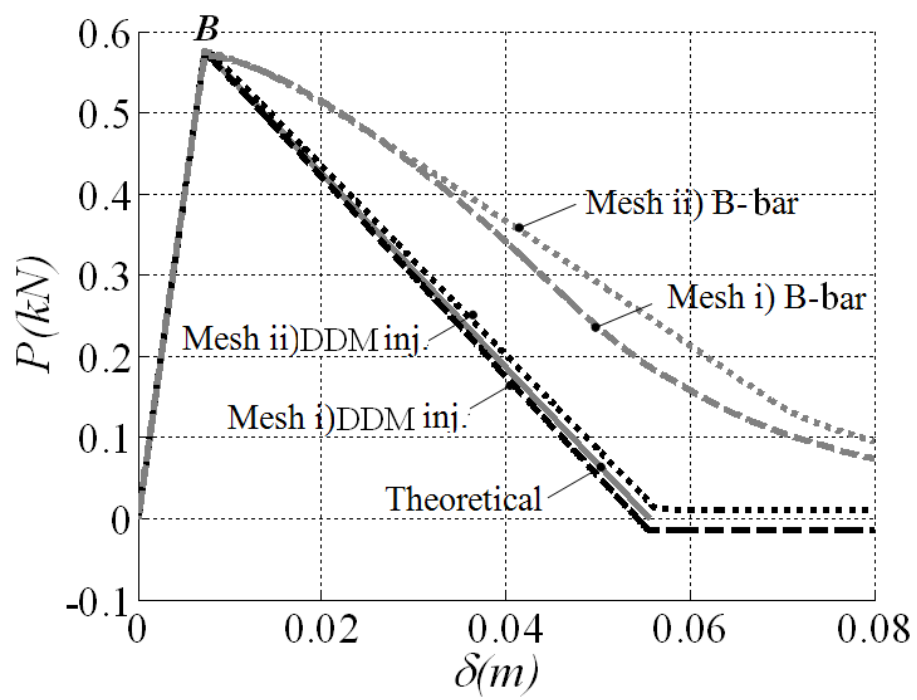

Figure 32 Strip subjected to tensile stretching.

Force-displacement curves.

In Figure 32, results are compared in terms of the force-displacement curves obtained with different strategies. It can be checked that 1) the results provided by the DDM injection improve very much the ones provided by the strain-localization based methodologies (B-bar), 2) they match almost perfectly the theoretical solution and 3) they are completely independent of the mesh-size and the mesh orientation.

(a)Mesh i)- DDM Injection

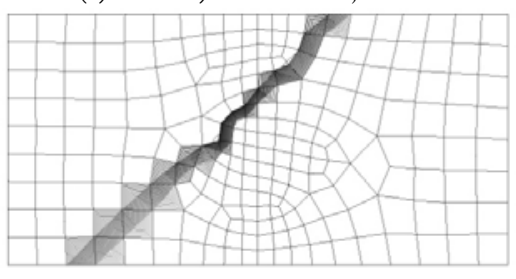

(c)Mesh i)- B-bar

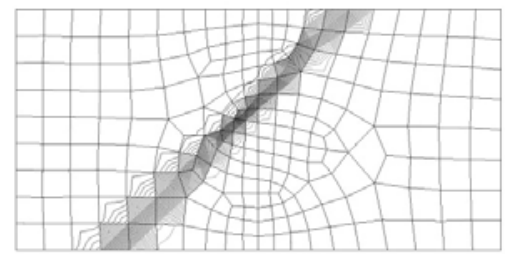

(b) Mesh ii)- DDM Injection

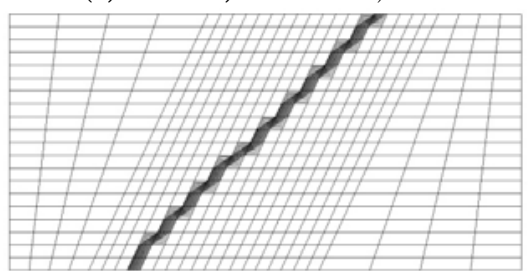

(d) Mesh ii)- B-bar

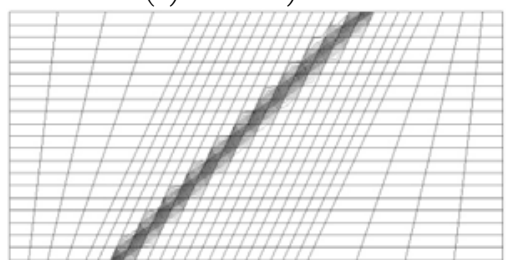

Figure 33. Strip subjected to tensile stretching. Iso-displacement contours

In Figure 33 it is clearly noticed that, unlike in the strain localization (B-Bar) formulation, the slip-line is sharply captured by the DDM injection technique in a one- 
element-width band, without spurious stress transfer to the neighboring elements, which unload elastically after the discontinuity is triggered. Figure 34 shows the deformed mesh for the DDM case. It can be observed that, consistently with the expected theoretical result (Oliver 2000), a perfect slip-line mode (exhibiting zero normal displacement jump) is obtained .

(a)

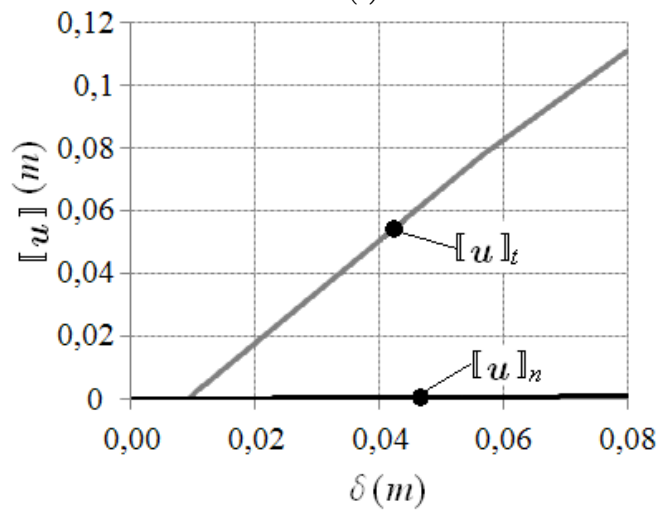

(b)

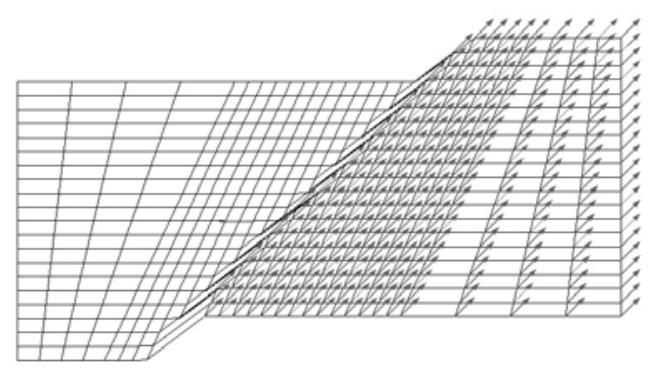

Figure 34. Strip subjected to tensile stretching - Mesh ii)- DDM Injection:

(a) displacement jump evolution (tangential, $\llbracket \boldsymbol{u} \rrbracket_{t}$, and normal, $\llbracket \boldsymbol{u} \rrbracket_{n}$, components), (b) Deformed mesh and displacement vectors.

In Figure 35 the injection domains, $\mathcal{B}_{\text {loc }}$ and $\mathcal{B}_{\text {dis }}$, are shown for the non-structured mesh case.

(a)

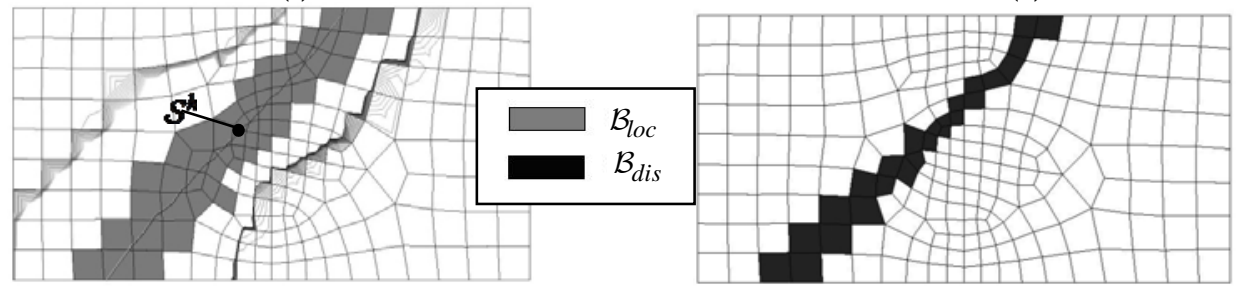

Figure 35. Strip subjected to tensile stretching. Injection domains, for two different times of analysis: (a) immediately after the bifurcation time (signaled with B in Figure 32, (b) after the discontinuous displacement mode injection.

Figure 35-(a), corresponds to a time immediately after the bifurcation point. The shaded elements display the domain $\mathcal{B}_{10 c}$, where the constant strain mode (CSM) is injected. The crack path $\mathcal{S}^{h}$, belonging to the zero level set of the crack-path field $\mu^{h}$, is also shown in that figure. At this time, the discontinuity domain, $\mathcal{B}_{\text {dis }}$, is empty. As soon as the discontinuous modes are injected ${ }^{20}$, those elements of $\mathcal{B}_{10 c}$ not containing the crack path $\left(\mathcal{B}^{(e)} \cap \mathcal{S}^{h}=\varnothing\right)$ automatically unload and leave the injection domain, which collapses into a single-element-width band of elements; all of them belonging to the discontinuity displacement mode (DDM) injection domain, $\mathcal{B}_{\text {dis }}$, shown in Figure 35-(b).

REMARK 5.1-1. For near-homogeneous problems, like the one focused here, the crack propagation is almost instantaneous. This is why the value of the $\bar{\gamma}$ parameter, generally recommended $\bar{\gamma}=0.95$ (see REMARK 4.2-5), can be set in this case, to $\bar{\gamma}=0.99$ without noticing any loss of robustness.

\footnotetext{
${ }^{20}$ Notice that this numerical example is a near-homogenous problem, for which the propagation of the localization band is almost instantaneous.
} 


\subsection{Isotropic continuum damage model: double cantilever beam (DCB) with diagonal loads}

The DCB test considered in section 3.3.5.1 is now retrieved using the proposed DDM injection. This example, described in Figure 36, provides a clear illustration of the evolution of the injection domains $\mathcal{B}_{l o c}$ (where the localized constant strain model, CSM, is injected) and $\mathcal{B}_{\text {dis }}$ (where the discontinuity displacement mode, DDM, is injected). The parameter $\bar{\gamma}$, establishing the threshold for the DDM injection, is set to $\bar{\gamma}=0.95$.

(a)

linear elastic fracture zone

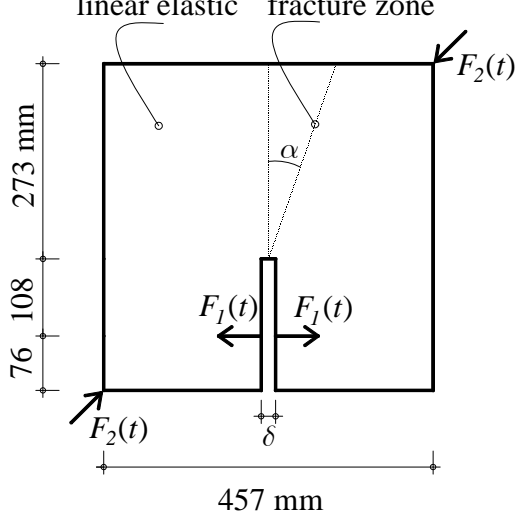

(b)

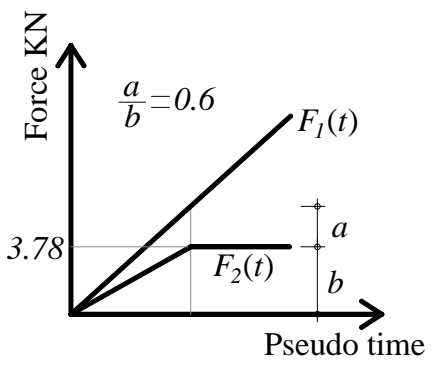

(c)

\begin{tabular}{cccc:c}
\hline $\begin{array}{c}\sigma_{u} \\
{[\mathrm{MPa}]}\end{array}$ & $\begin{array}{c}E \mathrm{GPa}] \\
{[3,0}\end{array}$ & $\boldsymbol{v}$ & $\begin{array}{c}G_{f} \\
{[\mathrm{~N} / \mathrm{m}]}\end{array}$ & $\bar{\gamma}$ \\
\hline 30,5 & 0,20 & 100 & 0.95 \\
\hline
\end{tabular}

Figure 36. Double cantilever beam with diagonal loads.

a) geometrical data, b) loading data, c) material properties of the isotropic damage model

In Figure 37 the evolution of injection domains is depicted. This evolution follows the scheme outlined in section 4.2.4. It can be observed a bulb-shaped domain, $\mathcal{B}_{\text {loc }}$, at the tip of the advancing localization band, where the material initially bifurcates (fulfilling condition in equation (36)) and remains in in-loading state, so that the constant strain mode (CSM) is injected. Soon after, most of the bifurcated elements behind the bulb unload (i.e. $\dot{\alpha}^{h}=0$ ) and leave the $\mathcal{B}_{\text {loc }}$ domain, according to equation (68)-(a), excepting for an inclined band, $\mathcal{B}_{\text {dis }}$, crossed by the crack path (shown in Figure 38), encompassing one element, which is injected the discontinuous displacement mode (DDM).

(a) $\mathrm{t}=1$

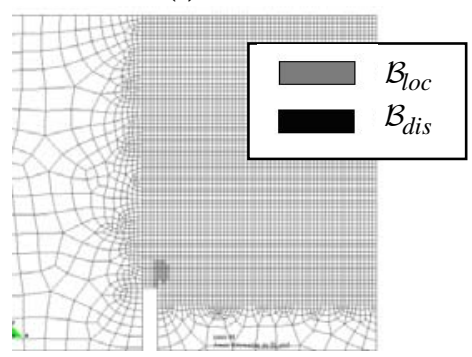

(c) $t=3$

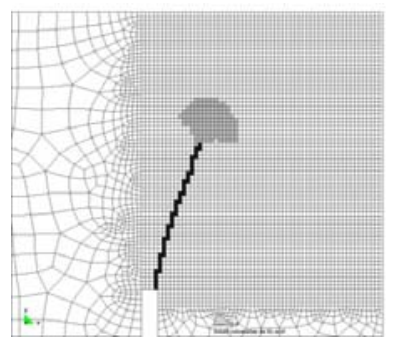

(b) $\mathrm{t}=2$

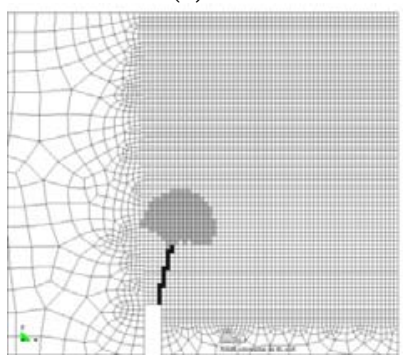

(d) $t=4$

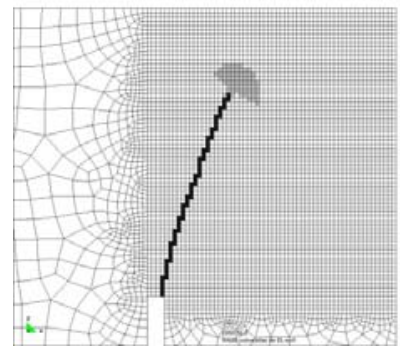

Figure 37. Double cantilever beam with diagonal loads. Evolution of the injection domains, $\mathcal{B}_{l o c}$ and $\mathcal{B}_{\text {dis }}$, at different stages of the analysis. 
(a) $t=2$

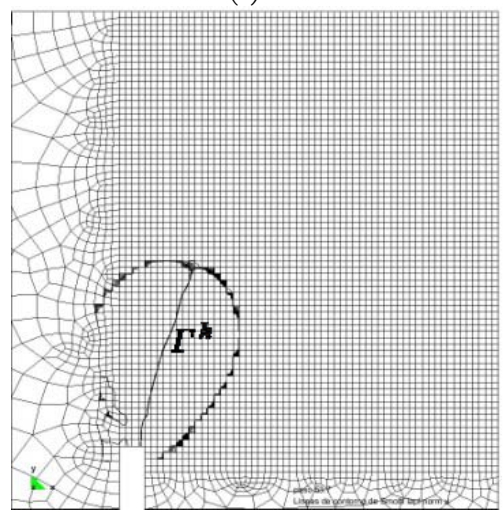

(b) $\mathrm{t}=4$

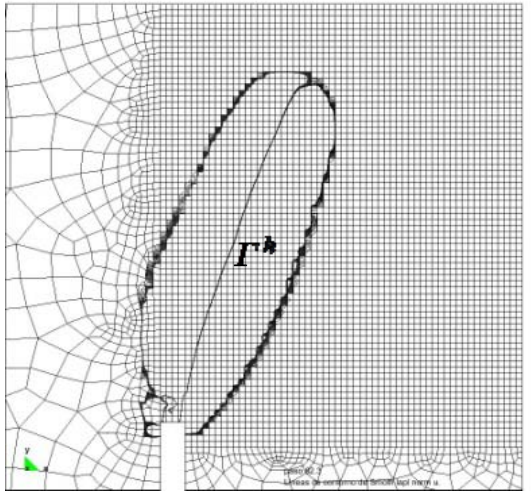

Figure 38. Double cantilever beam with diagonal loads.

Crack path set $\Gamma^{h}\left(\mu^{h}=0\right)$, at two different times of analysis.

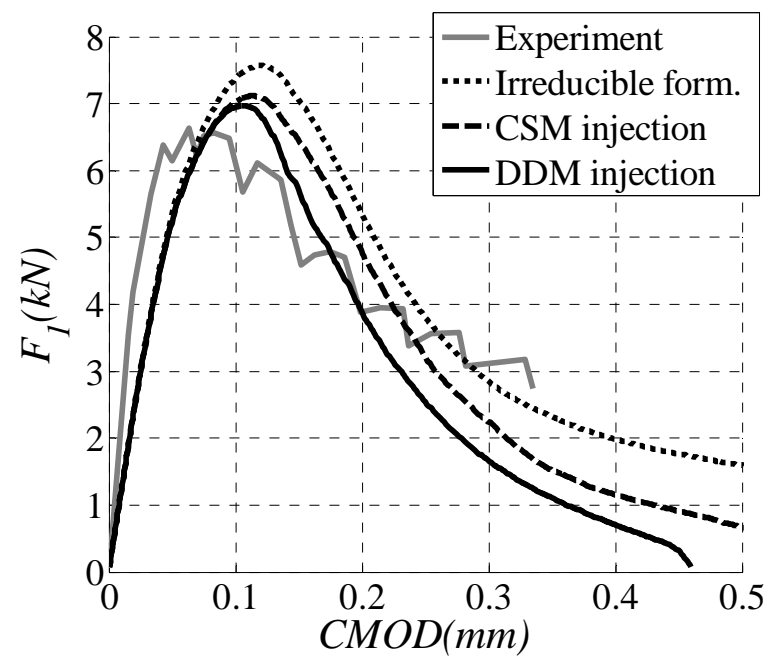

Figure 39. Double cantilever beam with diagonal loads

Force-displacement curves.

In Figure 39, the force-displacement curve obtained using DDM injection techniques is compared with results already presented in section 3.3.5.2 (see Figure 14). A significant reduction in terms of the dissipation (area below de curve) is observed in the DDM injection solution in comparison with the strain-localization-based alternatives. This fact, displays the stress locking minimization achieved by the proposed injection technique.

\subsection{Four-point bending test}

The classical four point bending test of a concrete beam, reported in (Arrea and Ingraffea 1982), is now considered. The geometry of the problem is depicted in Figure 40(a). The material behavior is modeled by a Rankine-type plasticity ${ }^{21}$ model whose parameters are summarized in Figure 40-(b). According to physical experiments, a fracture path, with curved trajectory, is expected to develop from the notch tip, as it is sketched in

${ }^{21}$ For the Rankine plasticity model a specific IMPL-EX integration scheme is derived in (Dias 2012; Dias, Oliver et al. 2012). 
Figure 40-(a). The numerical analysis was carried out by controlling the Crack Mouth Sliding Displacement (CMSD) in plane stress conditions.

(a)

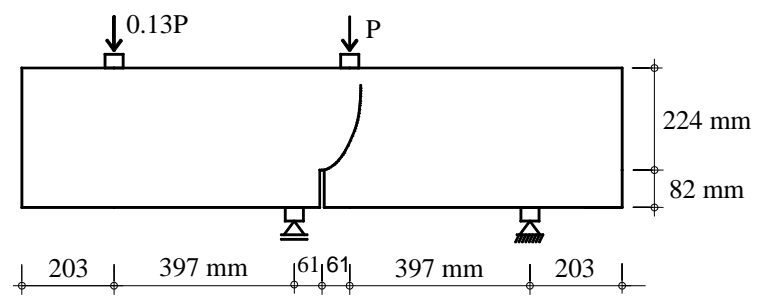

(b)

\begin{tabular}{cccc:c}
\hline $\begin{array}{c}\sigma_{y} \\
{[M P a]}\end{array}$ & $\begin{array}{l}E \\
\mathrm{GPa}]\end{array}$ & $v$ & $\begin{array}{c}G_{f} \\
{[N / m]}\end{array}$ & $\bar{\gamma}$ \\
\hline 2,8 & 24,0 & 0,18 & 100 & 0.95 \\
\hline \multicolumn{3}{c}{ Thickness $=0.156 \mathrm{~m}$}
\end{tabular}

Figure 40. Four-point bending test with Rankine-type plasticity:

(a) geometrical description, (b) material properties.

\subsubsection{Kinematic locking release.}

At this point, it is interesting to introduce the following topic: it is well known in the Continuum Strong Discontinuity Approach (CSDA) that the evolution of the displacement jump is constrained by the so-called strong discontinuity conditions. For most constitutive models, these conditions do not imply relevant restrictions, but, for some specific ones, they are restrictive in terms of the problems that can be effectively modeled. For instance, for $J 2$ plasticity models the strong discontinuity conditions establish that the normal component of the jump has to be zero, and only the tangential component, $\llbracket \dot{\boldsymbol{u}} \rrbracket_{t}$ can develop (pure mode II of fracture) (Oliver, Cervera et al. 1999). Consequently, only slipline type failure modes can develop (following straight or circular paths). Therefore, one has to be aware of these limitations when choosing the constitutive model to capture a specific failure mechanism. If the kinematics of the failure mechanism aimed at being modeled is not compatible with the restrictions stemming from the strong discontinuity conditions, this incompatibility can translate into unexpected locking effects here termed as kinematic locking 22 .

Another case of kinematic loading arises when Rankine-type plasticity models are used. Rankine-type plasticity is only compatible with development of the normal component of the jump, $\llbracket \boldsymbol{u} \rrbracket_{n}$, so that the tangential component has to be zero $\left(\llbracket \boldsymbol{u} \rrbracket_{t}=0\right)$. Consequently, locking-free propagation of a single fracture path under this fracture mode can only take place if the crack path is straight.

This is the case in the tackled problem. The onset of fracture at the crack tip occurs in Mode I, while in the stress-released part of the crack a mixed mode is experimentally observed (Galvez, Elices et al. 1998). Therefore, though a Rankine-type model seems to be a good choice to model the crack tip propagation, it is not suitable for modeling the mixedmode at the de-cohesive part of the crack, and, therefore spurious secondary cracks that lead to extra-dissipation can appear if a pure mode I is exactly imposed by the strong discontinuity kinematics. Here is where the strong discontinuity regularization referred to in sections 4.2.1 (see also Table 4.2.1-1) comes into play by releasing the aforementioned kinematic constraint. This can be checked in Figure 41, where evolution of the Crack Mouth Sliding Displacement (CMSD) with the applied load $P$ is depicted, for different values of the regularization factor parameter $\xi\left(\rightarrow k^{(e)}=\xi \ell^{(e)}\left(h^{(e)}\right)\right)$. The kinematic constraint $\left(\llbracket u \rrbracket_{t}=0\right)$ is strictly imposed if the regularization parameter, $k^{(e)}$ tends to zero for finite values of the finite element size $h^{(e)}(\xi \simeq 0$, see equation (61)). which translates into large degrees of

\footnotetext{
${ }^{22}$ This kind of "kinematic locking" stems from reasons completely different from the "numerical stress locking" referred to in REMARK 4.2-3 and analyzed in Appendix B.
} 
kinematic locking observed in the figure. On the contrary, for larger values of the parameter $\xi\left(\xi \cong 1,0\right.$, i.e.: the regularization parameter, $k^{(e)}$, is similar to the element size) the constraint $\left(\llbracket u \rrbracket_{t}=0\right)$ is only weakly imposed and the kinematic locking is almost completely released.

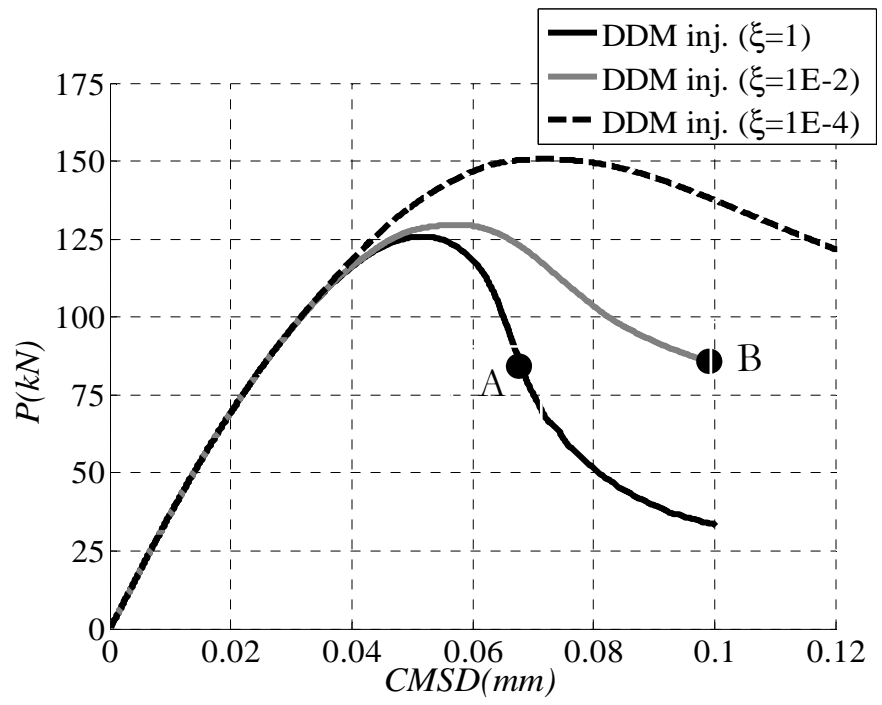

Figure 41. Four-point bending test with Rankine-type plasticity.

Force-displacement curves for different values of the regularization parameter $k^{(e)}=\xi^{(e)}$.

In Figure 42, the injection domains, at a representative time of the analysis are compared for both types of regularizations. For the small regularization parameter case, Figure 42-(a) shows the spurious in-loading bifurcated elements ( $\mathcal{B}_{\text {loc }}$ domain) at the bottom part of the injection domain, where spurious transversal cracking starts developing. Instead, for the large regularization parameter case (Figure 42-(b)) the spurious in-loading elements are almost negligible and the crack can further progress.

(a)

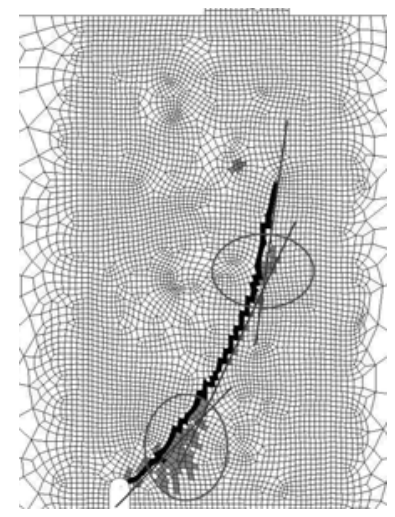

b)

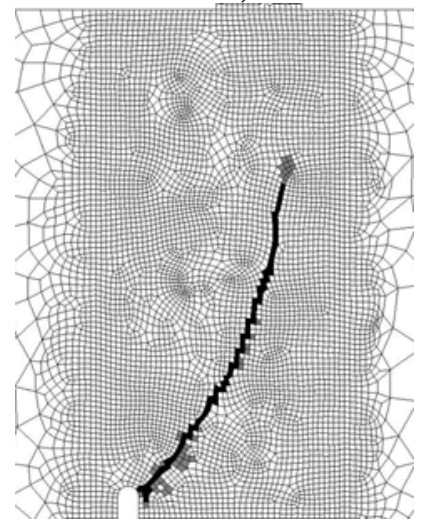

Figure 42. Four-point bending test with Rankine-type plasticity. Evolution of the injection domains: a) Small regularization parameter $\xi=0.01$ b) Large regularization parameter $\xi=1.0$ (plots corresponding, respectively, to points B and A of Figure 41).

These results show, the enormous benefits of large regularization parameters in minimizing the kinematic-locking effects (much less dissipative solution).

REMARK 5.3-1 It is well known that the four point bending test exhibits a mode I fracture mode at the tip of the crack, and a mixed fracture mode far 
from the crack tip (Galvez, Elices et al. 1998). In this sense, using a continuum Rankine plasticity model all along the crack path, as done here, could not seem an appropriate choice for modeling the total crack evolution, in front of alternative constitutive models as, for instance, continuum damage models (Oliver 2000), which are not kinematically constrained. However, the purpose of the study above is to show that even using an "imperfectly fitting model" (kinematically constrained, like the Rankine one), the resulting kinematic locking can be widely released in a simple manner: just by using larger values of the regularization parameter $(\xi \approx 1.0)$.

\subsubsection{Comparison of results in terms of the strain-injection technique}

In Figure 43, the effects and benefits of the proposed injection techniques are assessed. The Force vs. CMSD displacement curves, obtained using the classical (irreducible) formulation, the constant strain mode (CSM) injection and the discontinuous displacement mode (DDM) injection $(\xi=1.0)$, are compared.

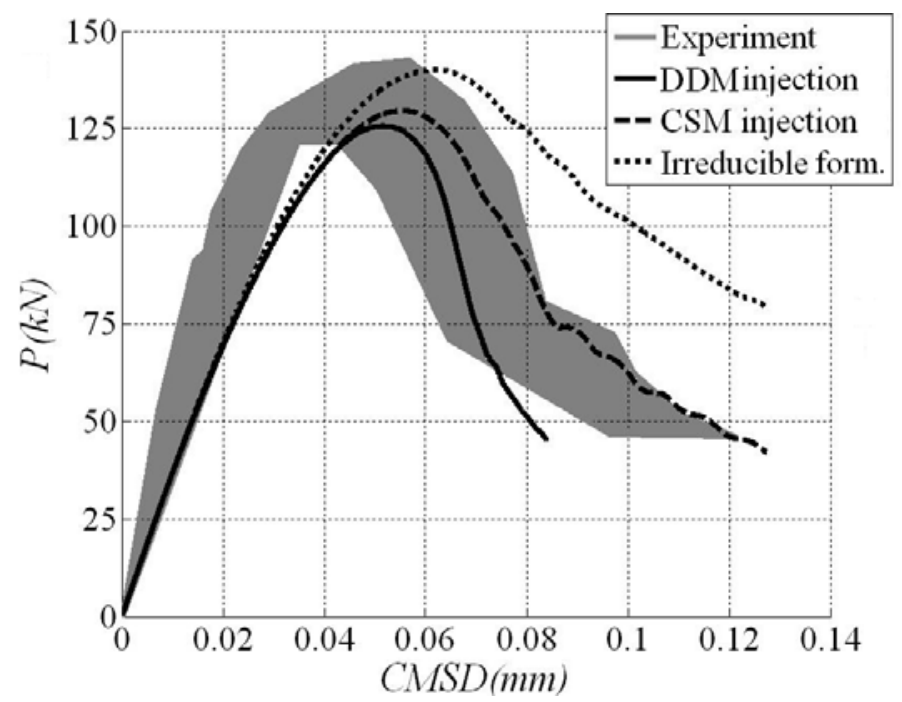

Figure 43. Four-point bending test with Rankine-type plasticity. Forcedisplacement curves.

The benefits of the injection procedures and, very specially, the minimal-dissipation character of the DDM injection are clearly displayed. As additional information the experimental envelop provided in (Arrea and Ingraffea 1982) (shaded zone) is also plotted $^{23}$.

\subsubsection{Crack-propagation issues}

In figure Figure 44 the strain-localized domains, in terms of the concentration of the displacement contours, for the DDM injection and the classical (irreducible formulation) procedure, are compared. Notice the sharpness of the localized domain for the DDM case (encompassing one element) in contrast with the classical solution (encompassing 2-3 elements). The additional elements across the band, in this last case, are responsible of the huge-extra dissipation observed in the structural response in Figure 43.

\footnotetext{
${ }^{23}$ this experimental envelop is not displayed for purposes of quantitative comparison of numerical vs. experimental results, but only for illustration of the "shape and size" of this envelop. As it is classical in this benchmark, some issues of the experimental set-up seem to be missed in the numerical modeling. Notice the drift of all numerical solutions, from the experimental band, at the initial (elastic) stage of the problem.
} 
(a)

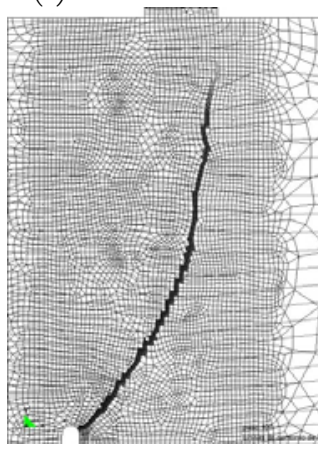

(b)

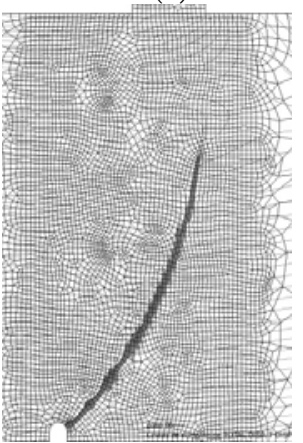

Figure 44. Four-point bending test with Rankine-type plasticity.

Iso-displacement contour plots: a) DDM injection, b) Irreducible formulation.

(a) $t=1$

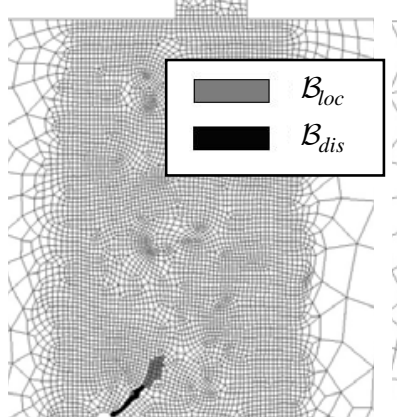

(b) $t=2$

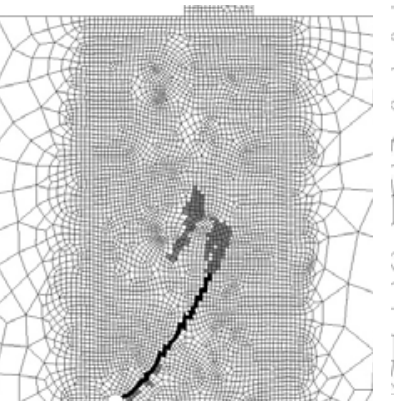

(c) $\mathrm{t}=3$

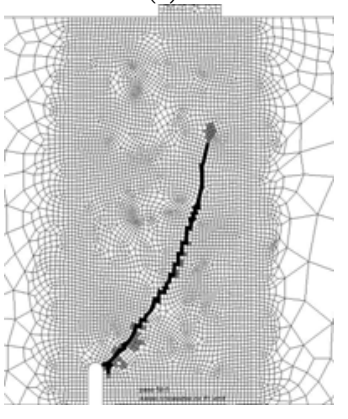

(d) $\mathrm{t}=4$

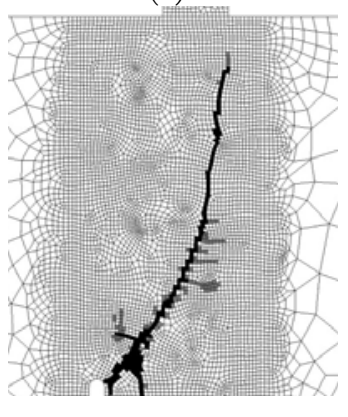

Figure 45. Four-point bending test with Rankine-type plasticity .

Evolution of the injection domains for different times of the analysis.

In Figure 45 the evolution of the injection domains is depicted for several stages of the analysis. Stage $t=4$ corresponds to the end of the analysis. At this stage, as the crack tip approaches the top of the beam some spurious cracking is also noticed at the bottom of the localization band. Notwithstanding, this effect is considered negligible since it just appears at the very end of the analysis.

(a) $t=2$

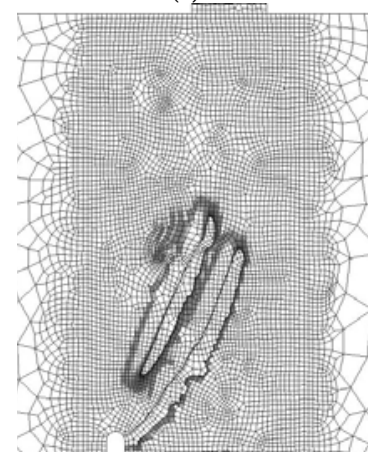

(b) $t=4$

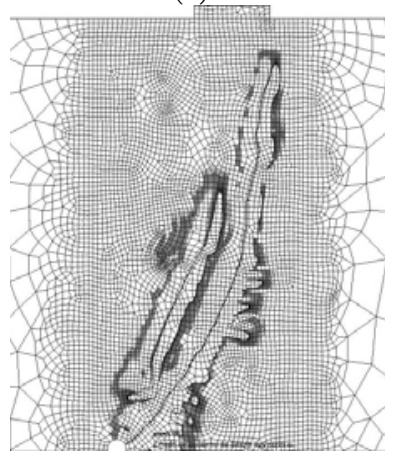

Figure 46. Four-point bending test with Rankine-type plasticity.

Crack path set, $\Gamma^{h}$, at two different times of the analysis

In Figure 46, the crack path set $\Gamma^{h}$, in BOX 2.2-1 (zero level set of the crack-path-field, $\left.\mu^{h}\right)$ is depicted. The central part of that set, clearly displays a single line, which is taken by 
the proposed techniques as the propagating crack path, $\mathcal{S}^{h}$ (notice that the DDM injection domain, $\mathcal{B}_{\text {dis }}$, in Figure 45 is clearly crossed by $\mathcal{S}^{h}$ in Figure 46.

In those figures it is also noticed a secondary crack path, discoursing parallel to the primary crack, corresponding to elements that at stage $t=2$ are crossed by an active crack. However in subsequent stages these elements unload elastically, leave the injection domain and come off $\mathcal{B}_{l o c}$ in Figure 45-(c)-(d). This is a secondary crack onset/arresting mechanism clearly captured by the simulation.

\subsubsection{Mesh refinement analyzes}

To study the finite element mesh-size influence, in Figure 47 and Figure 48 results obtained for three different meshes are presented. Similar solutions are obtained in all cases (Figure 47) in terms of crack propagation, whereas in terms of force-deflection curves convergence with mesh refinement and, therefore, mesh-size and mesh-bias objectivity of the obtained results are clearly shown (Figure 48).
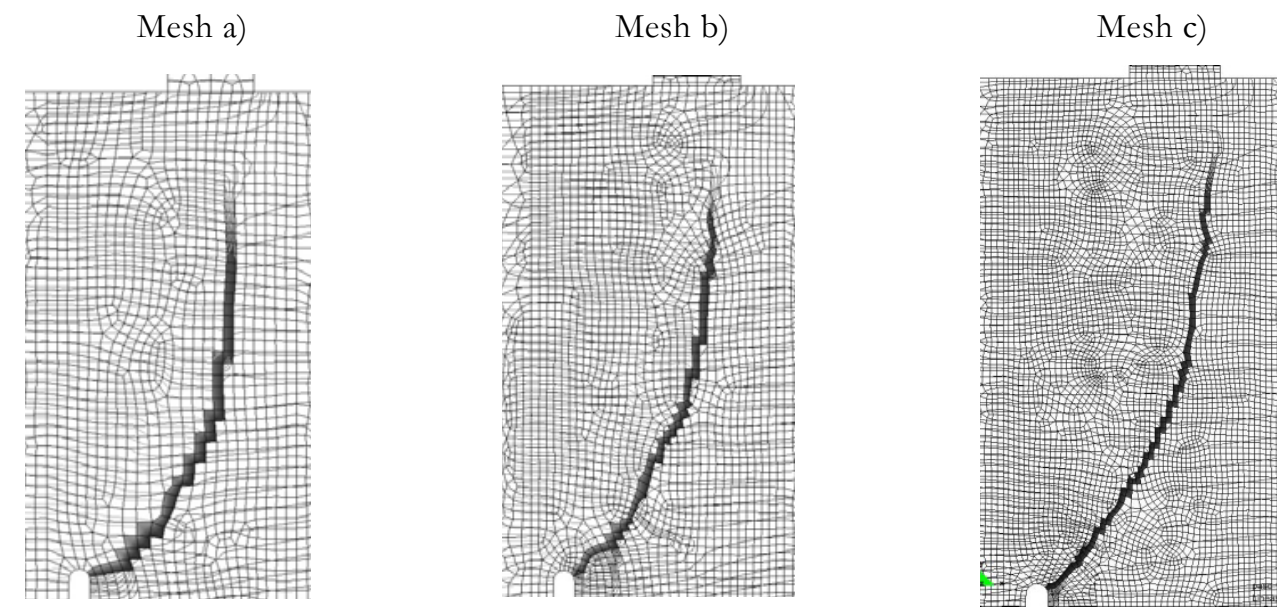

Figure 47. Four-point bending test with Rankine-type plasticity .

Iso-displacement contours for three different meshes

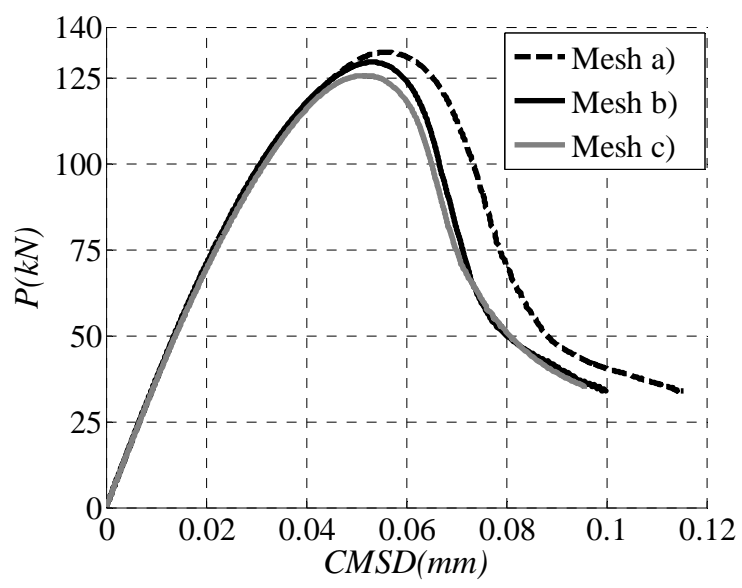

Figure 48. Four-point bending test with Rankine plasticity .

Force-displacement curves for three different meshes. 


\subsection{Slope stability problem using a $J_{2}$ plasticity model}

We switch now to benchmark problems exhibiting slip-lines. This kind of problems is typical in soil mechanics or metal extrusion problems (Regueiro and Borja 2001). The unstable response of a rigid indenter (footing), resting on an inclined soil embankment, modeled using a J2 plasticity model, is first analyzed. The geometrical and mechanical data are depicted in Figure 49.

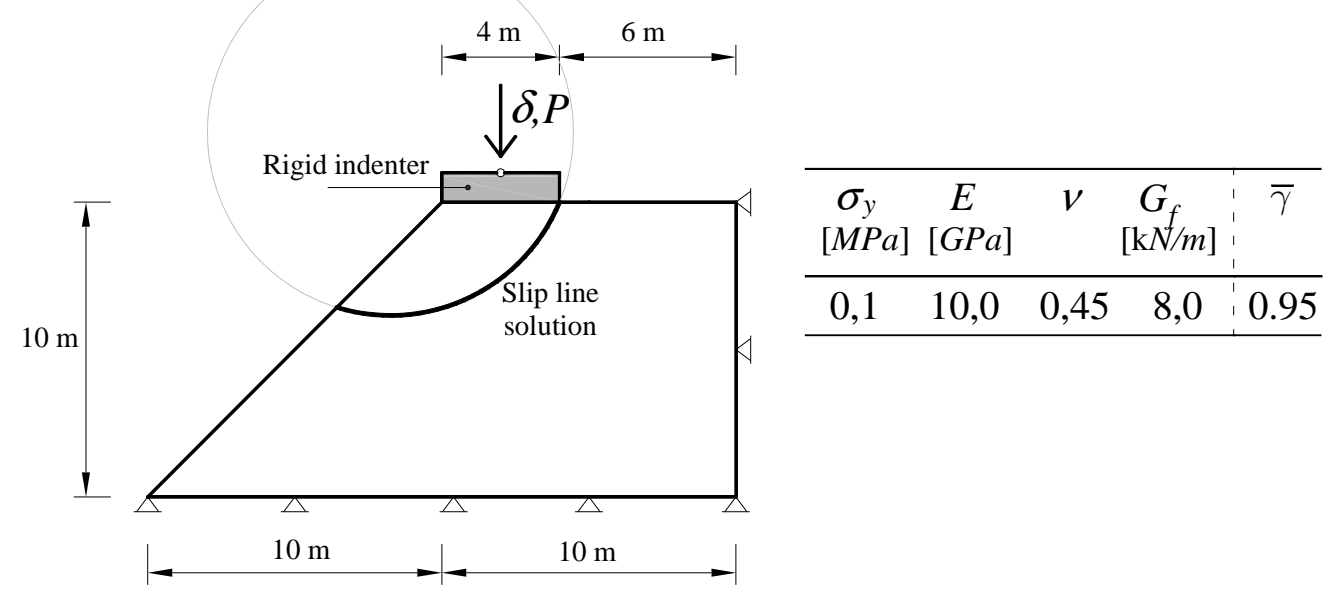

Figure 49. Slope instability problem using a J2 plasticity model.

Geometrical data and considered material properties

As commented in section 5.3.1, J2 plasticity models are compatible only with slip-linetype kinematics (mode II of fracture) and straight, or perfectly circular, slip-line paths (see Figure 49). Consequently, they are prone to provide results with kinematic-stress-locking.

In order to obtain a "reference solution" a finite element mesh "a-priori" adapted to the theoretical solution, consisting of a circular slip line passing through the rightmost footing corner, has been generated (see Figure 49 and Figure 50-(a)). For numerical assessment, a second mesh, completely misaligned with the theoretical slip-line, has been considered (see Figure 50-(b)-(c)).

In Figure 50, results obtained with the different simulation methodologies, are compared with those reference ${ }^{24}$ results. A large value of the regularization parameter $k^{e}(\xi=1,0)$ has been used in the discontinuity displacement mode (DDM) injection technique.

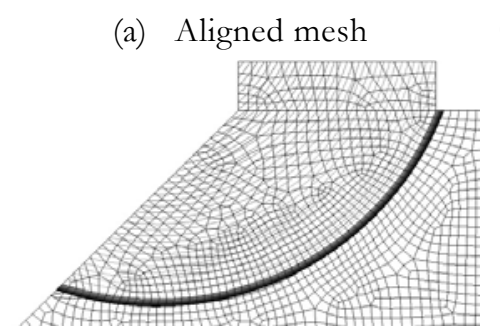

(b) Misaligned mesh (DDM Injection)

(c) Misaligned mesh (B-bar)

Figure 50. Slope instability problem using a J2 plasticity model.

Localization patterns (iso-displacement contours) for different solution strategies.

\footnotetext{
${ }^{24}$ Results obtained with the aligned mesh are almost coincident independently of the formulation employed (either B-bar or DDM injection). For that reason, they are condensed under the name of "aligned mesh".
} 
Again, sharp localization in a band encompassing one element is obtained, with the DDM injection, for the misaligned mesh (Figure 50-(b)). Instead, strain localization techniques make the displacement jump localize in a band encompassing several elements (Figure 50-(c)). This translates into stress-locking and a stiffer response in terms of the structural force-deflection response, depicted in Figure 51.

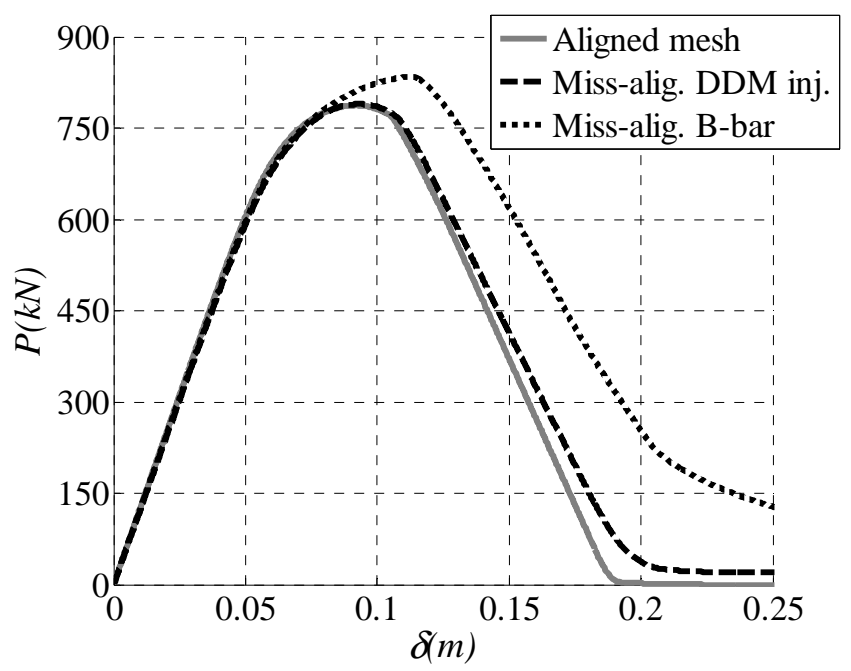

Figure 51. Slope instability problem using a J2 plasticity model.

Force vs. displacement curves for different solution techniques.

Notice the excellent agreement with the reference (aligned-mesh) solution provided by the DDM-injection technique .

(a) $\mathrm{t}=1$

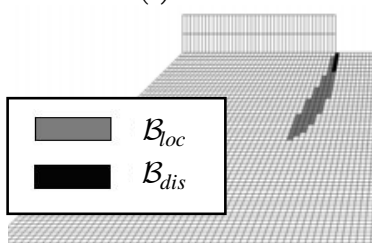

(b) $t=2$

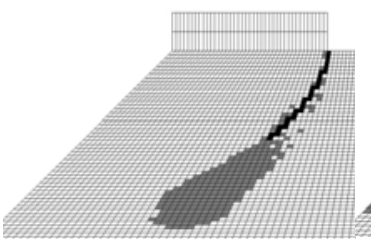

(c) $t=3$

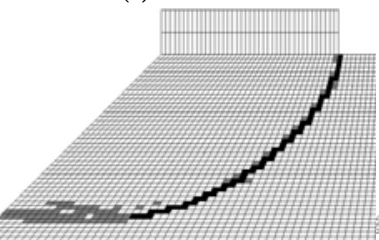

(d) $\mathrm{t}=4$

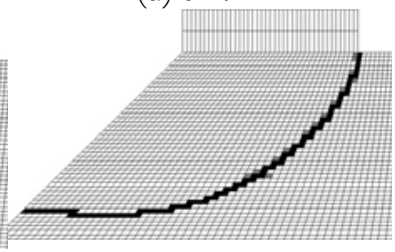

Figure 52. Slope instability problem using a J2 plasticity model.

Evolution of the injection domains along several stages of the analysis.

Figure 52 shows the evolution of the injection domains, at four representative stages of the analysis. It is observed, that once the discontinuity mode is injected, in $\mathcal{B}_{\text {dis }}$, the neighboring elements unload and leave the localization domain $\mathcal{B}_{l o c}$. Eventually, the slip line is captured by the single-element-width band of injected elements following an almost perfect circular path (Figure 52-(d)). This is corroborated in Figure 53 displaying the crack path $\mathcal{S}^{h}$, captured by the proposed crack-path-field technique, at an intermediate stage (Figure 53-(a)) and at the final stage of the analysis (Figure 53-(b)). Notice, by comparison with the corresponding stages in Figure 52, that the crack-path is anticipated long in advance of what is required for the injection of the DDM mode. 
(a) $t=2$

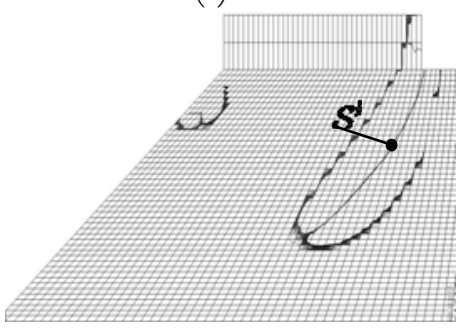

(b) $t=3$

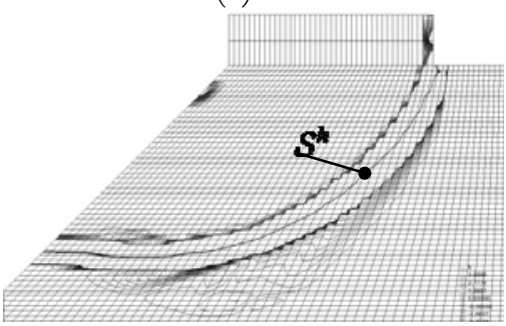

Figure 53 . Slope instability problem using a J2 plasticity model.

Propagating crack path, $\mathcal{S}^{h}$, at two different stages of the analysis.

\subsection{Prandtl's problem resolution using a $J_{2}$ plasticity model}

The numerical example presented in this section corresponds to the classical soil mechanics problem of an undrained soil domain undergoing a central footing (see Figure 54). The analytical solution of the problem has been often considered a benchmark (Chen 1975; Oliver, Cervera et al. 1999)) and consists of a rigid and rough surface footing (indenter) acting on a large (theoretically indefinite) soil domain, where the Prandtl's failure mechanism, consisting of a number of slip lines (Mode II of fracture), are expected to develop (see Figure 54).

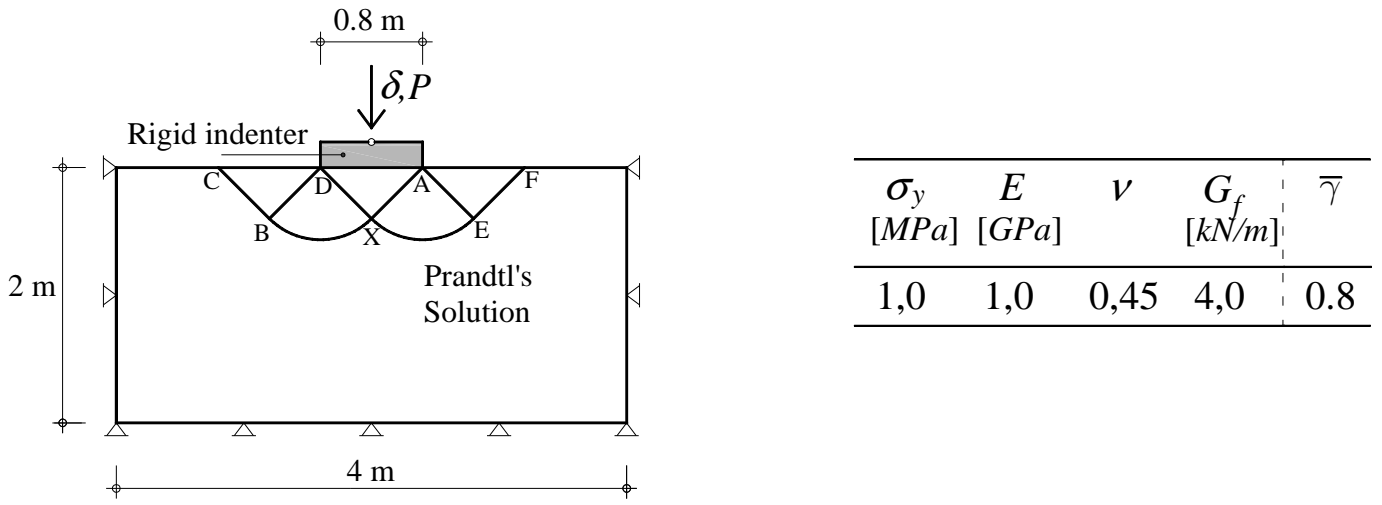

Figure 54. Prandtl's problem resolution using a J2 plasticity model.

Geometrical data and considered material properties.

\subsubsection{Discontinuous displacement mode (DDM) injection solution}

In Figure 55, the force-displacement curves, either considering the B-bar methodology or the proposed DDM injection technique are plotted). Again, as it can be checked in the figure, DDM injection supplies a much less dissipative response (stress locking-free) when compared with a standard strain-localization technique (B-Bar method). 


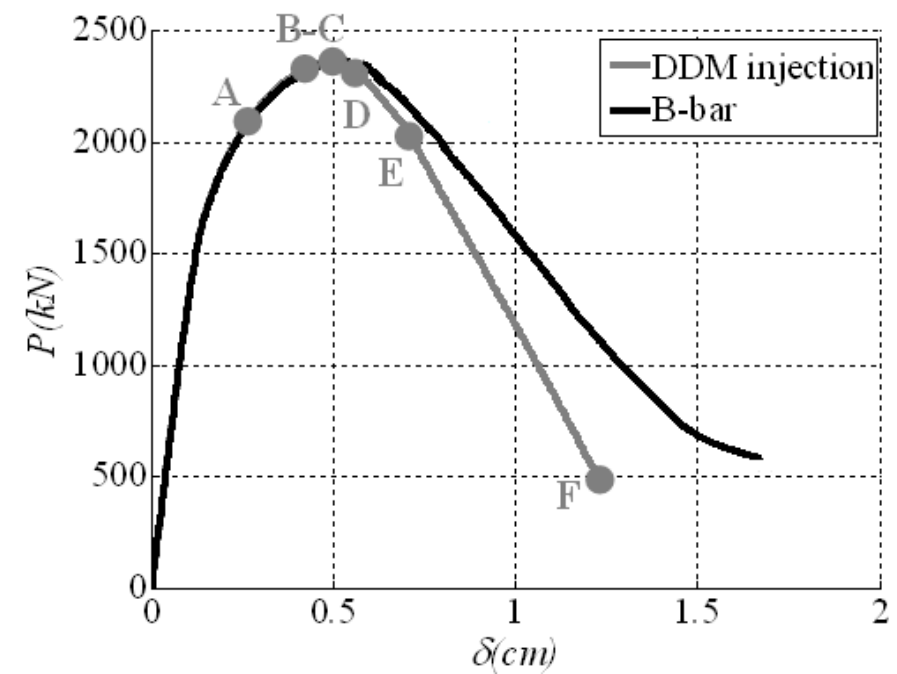

Figure 55. Prandtl's problem resolution using a J2 plasticity model.

Force-displacement curves (labeled points correspond to time-stages depicted in Figure 56).

In the following, the failure mechanism obtained with the DDM injection technique is described.

(a)-Point A

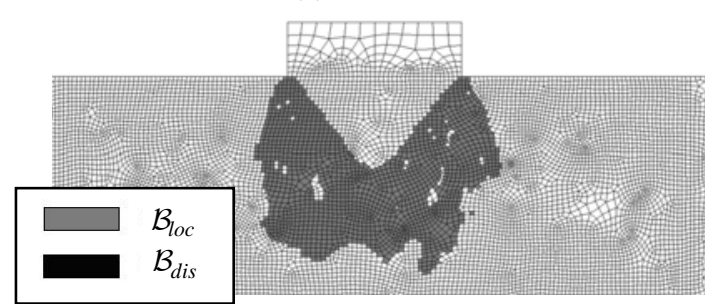

(c)-Point C

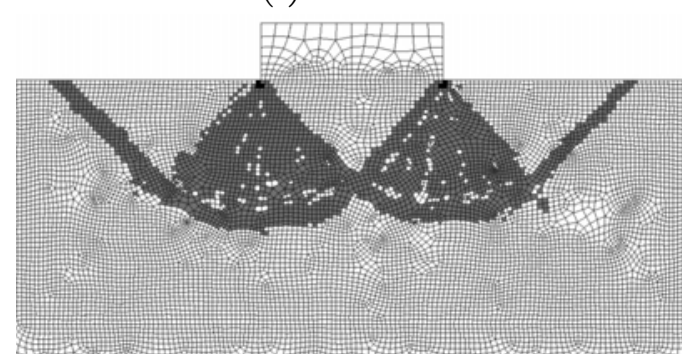

(e)-Point E

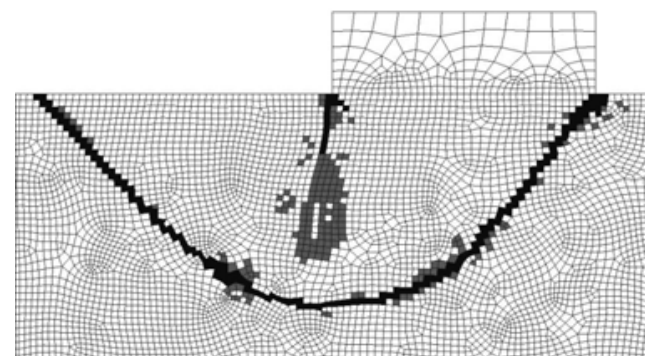

(b)-Point B

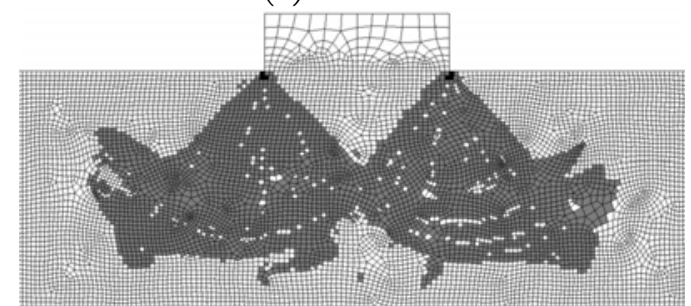

(d)-Point D

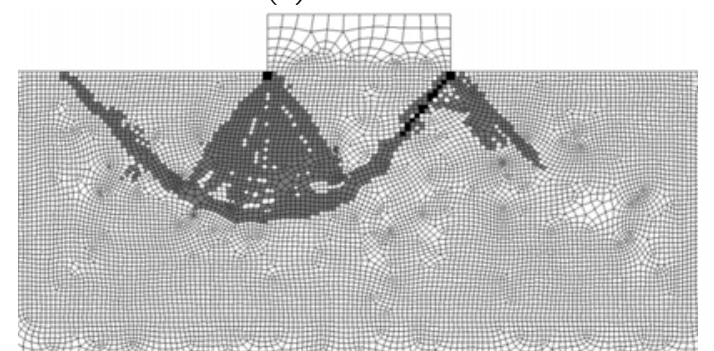

(f)-Point F

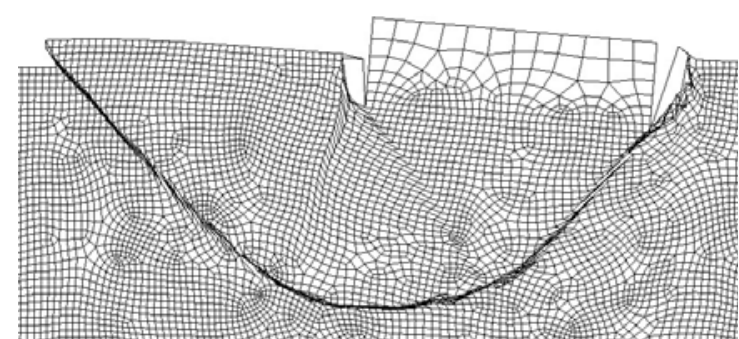

Figure 56. Prandtl's problem resolution using a J2 plasticity model.

From (a) to (e ): evolution of the injection domains at points in Figure 55.

(f) Deformed (amplified) mesh at the end of the analysis showing the final failure mechanism 
Yielding initiates at the bottom corners of the footing, at early stages of the loading process, and spreads, in a diffuse manner (Figure 56-(a)). A triangular wedge of soil, beneath the footing, starts moving downwards vertically (jointly with the rigid footing) without any plastic deformation.

As loading progresses, the yielding zones keep spreading, now laterally, towards the sides of the footing (see Figure 56-(b) and (c)). In step C the symmetric Prandtl's failure mechanism, is already formed: the central triangular wedge beneath the footing induces an upward movement of two lateral wedges which slide with respect to the adjacent soil layers. The triangle wedges moving upwards also remain elastic as it can be seen in Figure 56-(c). This symmetric mechanism is unstable ${ }^{25}$, i.e. small numerical perturbations, as those arising from numerical perturbations of the symmetry (note that an un-symmetric mesh is used), will trigger the solution collapse into an unsymmetrical failure mechanism trough a near-bifurcation process. This leads to the pattern displayed in Figure 56-(d). Is at this time when the unsymmetrical failure mechanism starts developing: the rightmost plastic wedge unloads elastically and the footing rotates.

It is noticeable that, until this point, the failure process is almost fully dominated by strain localization modes. The discontinuity injection domain $\mathcal{B}_{\text {dis }}$ is just a minimal part of a relatively large injection domain, $\mathcal{B}_{i n j}$. Beyond point $\mathrm{D}$, the unsymmetrical failure mechanism persists, in a stable manner, along points D-E-F of Figure 55 until the end of the analysis. However, the injection mechanism evolves substantially: a unique straightcircular-straight slip-line propagates from the right most corner of the footing translating into a rigid body motion of the footing and the soil beneath. Along this bifurcation process, the power of the proposed crack-path-field technique is strongly challenged. Figure 57 shows as it anticipates, successfully and much in advance, the slip-line path $\mathcal{S}^{h}$ to be subsequently injected with a DDM mode.

(a)

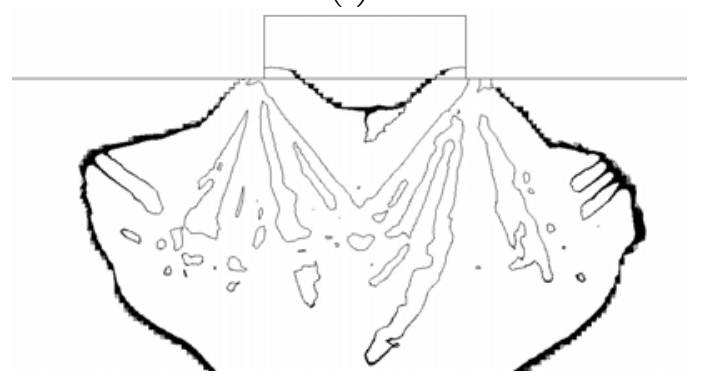

(b)

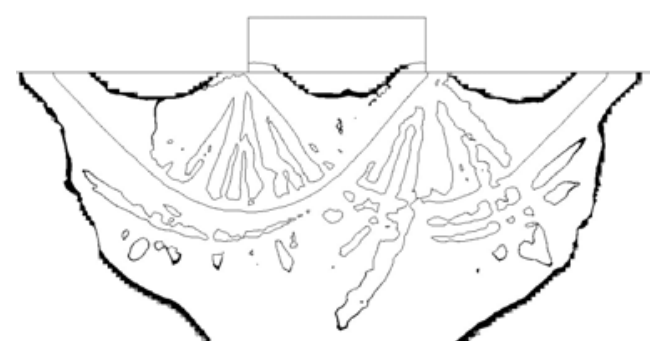

Figure 57. Prandtl's problem resolution using a J2 plasticity model.

Zero level set of the crack path field $\Gamma^{h}\left(\mu^{h}=0\right)$ corresponding to: (a) point-B , and (b) point $\mathrm{D}$ in Figure 55.

REMARK 5.5-1. The authors would like to remark the specific challenges posed by this problem, that emerge from its symmetry (displayed in the Prandtl's mechanism in Figure 54 and Figure 56-(c). This failure mechanism is essentially unstable, since, at point $\mathrm{C}$ of Figure 55, any infinitesimal perturbation unfolds the symmetry and leads to the, less dissipative, singleslip-line mechanism displayed in Figure 56-(d). In consequence, if a large value of the parameter $\bar{\gamma}$ are used (thus injecting the DDM before the

\footnotetext{
${ }^{25}$ in contrast, the analytical Prandtl's solution is obtained under the "slip-line-theory" that assumes perfect plasticity (no strain-softening). Therefore, the symmetric mechanism is "un-physically" stable and provides only an upper bound of the critical load.
} 
bifurcation unfolding via the perturbation produced by the finite element mesh) the proposed crack-path-field technique is unable to discriminate between the two symmetric slip lines. However, using values of $\bar{\gamma}$ slightly smaller than usual (see REMARK 4.2-5), delays the DDM injection beyond the bifurcation unfolding, and the unsymmetric single-slip-line failure mechanism, in Figure 56-(e-f), can be captured at no problem.

\subsubsection{Mesh dependency analysis}

Figure 58 compares the obtained failure mechanism for three different, increasingly refined, meshes in terms of the displacement contours that signal the slip-line path. There, it can be checked that they are, essentially, the same. In quantitative terms the forcedeflection solutions, in Figure 59, obtained for the three meshes, confirm the mesh objectivity character of the solutions provided by the proposed methodology.
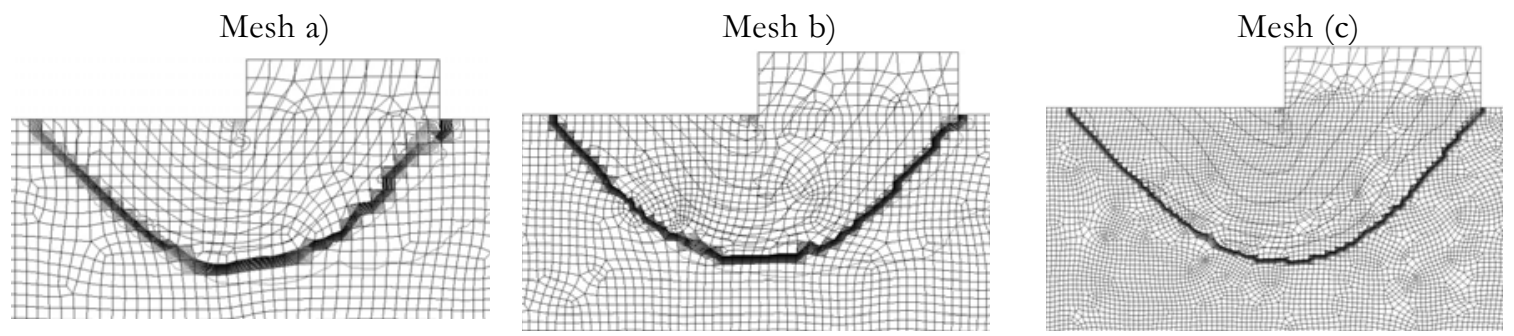

Figure 58. Prandtl's problem resolution using a J2 plasticity model.

Iso-displacement contours for three, increasingly refined, meshes.

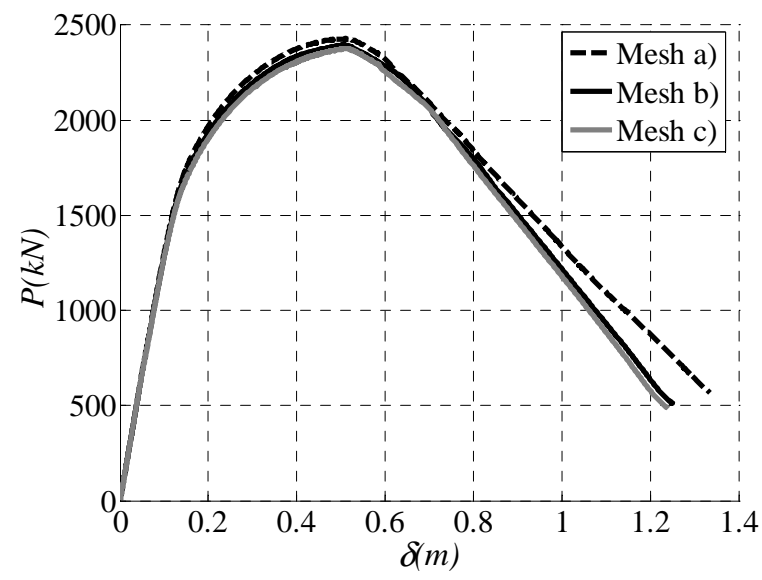

Figure 59. Prandtl's problem resolution using a J2 plasticity model.

Force-displacement curves for three different meshes in Figure 58.

\section{Concluding remarks}

Along this work, two new techniques for propagating material failure modeling have been presented:

1. The so-called crack-path-field technique to identify the crack-path represented by a strain localization-based solution of a material failure problem.

2. Strain-injection techniques to insert, in selected specific domains, appropriate strain field with two goals: 1) provide a reliable strain localization field, to be used in the 
context of the crack-path-field technique and 2) capture and model the discontinuous displacement fields arising from material failure (cracks and sliplines) overcoming classical flaws, typically mesh-bias dependencies and stress locking, in alternative procedures.

The combination, in a staggered manner, of both techniques provides a new numerical method for simulation of propagating material failure. It has been assessed by its application to a comprehensive variety of benchmarks, and the following conclusions have been obtained:

- The proposed methodology effectively overcomes classical drawbacks of standard strain-based localization methods for propagating material failure. The results obtained in all tested cases show no spurious mesh dependence, even when tested in combination with very demanding meshes, and minimize, to almost full elimination, the stress-locking effects.

- Its character is completely general. It is based on the use of the Continuum Strong Discontinuity approach (CSDA) and, therefore, the material is modeled using a strain-stress-type constitutive equation, with no other restriction than being equipped with strain softening regularized in terms of the fracture energy. In principle, any family of internal-variable-based local constitutive models (plastic models, continuum damage models etc.) can be used with no other limitation than their physical appropriateness for the considered problem.

- The method is minimally invasive as for the simulation code in which it is implemented. Any standard finite element code for non-linear solid mechanics analyses can be equipped with the proposed finite element technology at the cost of some modifications to affect essentially the "element level". Typically those modifications are (for the considered case of quadrilateral -bilinear finite elements) the following:

- Addition of two extra sampling points at the center of the element (section 4.2.4.1)

- Storage of one additional effective stress field at every regular sampling points (section 4.2.4.2 and BOX 4.2-2)

- An algorithm for tagging the elements of the injection domains $\mathcal{B}_{\text {loc }}$ and $\mathcal{B}_{\text {dis }}$ (section 4.2.4).

- A specific algorithm, based on a double smoothing procedure, to determine the crack-path-field at the nodes of the injected elements (BOX 2.2-1 and Appendix A.3), and select the sides crossed by the crack-path.

- Inclusion of an analytical solution or a numerical algorithm for determining the bifurcation time and the bifurcation directions (Oliver, Huespe et al. 2010)).

- Inclusion of the strain field corresponding to the discontinuous displacement mode BOX 4.2-2 and Appendix A)

- Condensation of the internal discontinuous displacement mode (Appendix A.2).

- The resulting implementation exhibits the following features:

- Conceptual simplicity: the method keeps for the user the apparent format and, therefore, the simplicity of classical strain-localization methods.

- Gets rid of cumbersome, and code-invasive crack-tracking algorithms, i.e. propagating level-set methods and global tracking algorithms (Stolarska, Chopp et al. 2001; Oliver, Huespe et al. 2004 ) with almost no apparent cost in terms of its accuracy and robustness. 
- Low computational cost: since the additional discontinuous degrees of freedom are condensed at element level, the computational cost is kept in the order of standard finite element methods.

- The method can be effectively used in combination with very coarse finite element meshes. In contrast with other methods aiming at capturing propagating cracks, which require several elements across a band to capture the discontinuity, e.g. phase-field models (Miehe, Welschinger et al. 2010), the proposed approach is an intra-element method. Consequently, only one-element-width band (no matter the element size) is used to capture the discontinuity. Therefore, actually coarse meshes can be used and local mesh refinement is not needed. This reduces enormously the final computational cost of the analysis.

So far, the, method has only been implemented in 2D cases (tough its extension to 3D cases seems straightforward) and on the basis of quadrilateral elements.

In addition, the assessment of its application field has been limited, in this work, to quasi-static problems of quasi-brittle fracture. Its ability to capture some failure mechanisms appearing in fast propagating dynamic fracture, as crack branching, and the extension to the three-dimensional case, and to other families of finite elements has to be object of additional investigation.

\section{ACKNOWLEDGEMENTS}

The research leading to these results has received funding from the European Research Council under the European Union's Seventh Framework Programme (FP/2007-2013) / ERC Grant Agreement n. 320815, Advanced Grant Project COMP-DES-MAT.

Financial support from the Spanish Ministry of Science and Innovation, and the Catalan Government Research Department, under research grants BIA2011-24258 and 2009 SGR 1510 , respectively, is also acknowledged.

Financial support from Fundação para a Ciência e a Tecnologia (FCT), under research Grant SFRH/BD/41426/2007, and Laboratório Nacional de Engenharia Civil (LNEC), is also acknowledged. 


\section{Appendix A. Implementation issues}

\section{A.1 Numerical time integration and quadrature of the mechanical residue}

After the statements in section 4.2.4.2 the residual rate of the mechanical problem, $\dot{\boldsymbol{R}}_{\text {mech }}$, stemming from equations (39) and (65), using Voigt's notation, reads:

$$
\begin{aligned}
& \dot{\boldsymbol{R}}_{\text {mech }}\left(\dot{\hat{\boldsymbol{u}}}^{h}(t), \mathcal{B} \backslash \mathcal{B}_{\text {inj }}(t), \mathcal{B}_{\text {loc }}(t), \mathcal{B}_{\text {dis }}(t)\right)=
\end{aligned}
$$

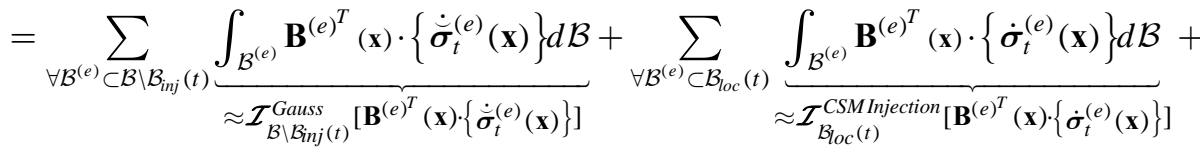

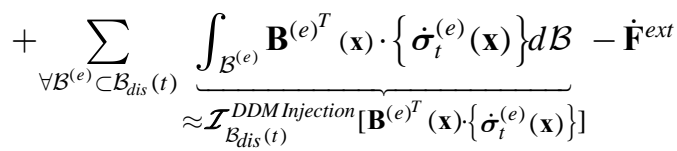

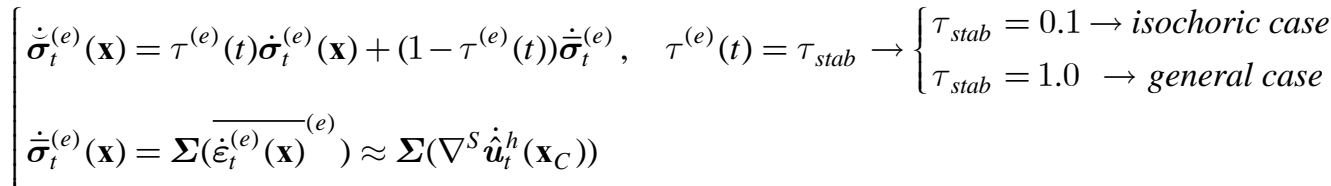

$$
\begin{aligned}
& \left\{\dot{\boldsymbol{\sigma}}_{t}^{(e)}(\mathbf{x})=\Sigma\left(\dot{\varepsilon}_{t}^{(e)}(\mathbf{x})\right)\right. \\
& \int \dot{\varepsilon}_{t}^{(e)}(\mathbf{x})=\nabla^{S} \dot{\hat{\boldsymbol{u}}}_{t}^{h}(\mathbf{x}) \quad, \mathcal{B}^{(e)} \subset \mathcal{B} \backslash \mathcal{B}_{\text {dis }}(t) \\
& \left\{\dot{\varepsilon}_{t}^{(e)}(\mathbf{x})=\nabla^{S} \dot{\hat{\boldsymbol{u}}}_{t}^{h}(\mathbf{x})+\left(\left(\frac{\mu_{\mathcal{S}}^{(e)}(\mathbf{x})}{k^{(e)}} \mathbf{n}^{(e)}-\left(\nabla \varphi_{t}^{(e)}(\mathbf{x})\right) \otimes \llbracket \dot{\boldsymbol{u}} \rrbracket_{t}^{(e)}\right)^{S}, \mathcal{B}^{(e)} \subset \mathcal{B}_{\text {dis }}(t)\right.\right.
\end{aligned}
$$

where the dependence on time of $\dot{\boldsymbol{R}}_{\text {mech }}$, trough the integration domains, $\mathcal{B} \backslash \mathcal{B}_{\text {inj }}(t), \mathcal{B}_{\text {loc }}(t)$ and $\mathcal{B}_{\text {dis }}(t)$ is emphasized ${ }^{26}$. In equation A.(1), $\mathbf{F}^{\text {ext }}\left(\mathbf{b}, \mathbf{t}^{*}\right)$ stands for the external forces, computed in a regular manner in terms of the body forces, $\mathbf{b}$, and the boundary tractions, $\mathbf{t}^{*}, \mathbf{B}^{(e)}(\mathbf{x})$ is the standard elemental deformation matrix for the four-node quadrilateral element and bracketed notation $\{(\bullet)\}$ is used to denote the vector (Voigt's notation) form of the symmetric second order entity (•). The stabilized stresses $\boldsymbol{\sigma}_{t}^{(e)}(\mathbf{x})$ in A.(1) have been introduced to account for a possible isochoric case in $\mathcal{B} \backslash \mathcal{B}_{\text {inj }}(t)$ (see equation (39)).

Also in equation A.(1) the functionals $\mathcal{I}_{\mathcal{B} \backslash \mathcal{B}_{i n j}(t)}^{\text {Gaus }}\left[\mathbf{B}^{(e) T}(\mathbf{x}) \cdot\left\{\dot{\tilde{\boldsymbol{\sigma}}}_{t}^{(e)}(\mathbf{x})\right\}\right]$, $\mathcal{I}_{\mathcal{B}_{\text {loc }}(t)}^{\text {CSM Injection }}\left[\mathbf{B}^{(e) T}(\mathbf{x}) \cdot\left\{\dot{\boldsymbol{\sigma}}_{t}^{(e)}(\mathbf{x})\right\}\right]$ and $\mathcal{I}_{\mathcal{B}_{\text {dis }}(t)}^{\text {DDM Injection }}\left[\mathbf{B}^{(e) T}(\mathbf{x}) \cdot\left\{\dot{\boldsymbol{\sigma}}_{t}^{(e)}(\mathbf{x})\right\}\right]$ are defined as the numerical quadratures of the corresponding kernels using, respectively, the regular four-point Gauss quadrature, $\mathbf{x}_{i}, i=(1,2,3,4)$, the barycenter-injection-point quadrature, $\mathbf{x}_{C} \equiv \mathbf{x}_{\mathcal{S}}$, and the two-injection-point quadrature defined in Table 4.2.1-1, $\mathbf{x}_{\mathcal{S}}$ and $\mathbf{x}_{\mathcal{B} \backslash \mathcal{S}}$, i.e.:

$$
\begin{aligned}
& \mathcal{I}_{\mathcal{B} \backslash B_{i n j}(t)}^{\text {Gaus }}\left[\mathbf{B}^{(e)^{T}}(\mathbf{x}) \cdot\left\{\dot{\boldsymbol{\sigma}}_{t}^{(e)}(\mathbf{x})\right\}\right]=\sum_{i=1}^{i=4} \mathbf{B}^{(e)^{T}}\left(\mathbf{x}_{i}\right) \cdot\left\{\dot{\boldsymbol{\sigma}}_{t}^{(e)}\left(\mathbf{x}_{i}\right)\right\} w_{i}\left(\mathbf{x}_{i}\right) \\
& \mathcal{I}_{B_{\text {loc }(t)}}^{\text {CSM Injection }}\left[\mathbf{B}^{(e)^{T}}(\mathbf{x}) \cdot\left\{\dot{\boldsymbol{\sigma}}_{t}^{(e)}(\mathbf{x})\right\}\right]=\mathbf{B}^{(e)^{T}}\left(\mathbf{x}_{C}\right) \cdot\left\{\dot{\boldsymbol{\sigma}}_{t}^{(e)}\left(\mathbf{x}_{C}\right)\right\} \operatorname{meas}\left(\mathcal{B}^{(e)}\right) \\
& \mathcal{I}_{B_{\text {dis }}(t)}^{\text {DDMInjection }}\left[\mathbf{B}^{(e)^{T}}(\mathbf{x}) \cdot\left\{\dot{\boldsymbol{\sigma}}_{t}^{(e)}(\mathbf{x})\right\}\right]=\mathbf{B}^{(e)^{T}}\left(\mathbf{x}_{\mathcal{B} \backslash \mathcal{S}}\right) \cdot\left\{\dot{\boldsymbol{\sigma}}_{t}^{(e)}\left(\mathbf{x}_{\mathcal{B} \backslash \mathcal{S}}\right)\right\} W\left(\mathbf{x}_{\mathcal{B} \backslash \mathcal{S}}\right)+ \\
& +\mathbf{B}^{(e)^{T}}\left(\mathbf{x}_{\mathcal{S}}\right) \cdot\left\{\dot{\boldsymbol{\sigma}}_{t}^{(e)}\left(\mathbf{x}_{\mathcal{S}}\right)\right\} W\left(\mathbf{x}_{\mathcal{S}}\right)
\end{aligned}
$$

where $w\left(\mathbf{x}_{i}\right), i=(1,2,3,4)$ are the weights corresponding to the four-point Gauss integration, meas $\left(\mathcal{B}^{(e)}\right)$ the weight corresponding to the barycenter-injection point and $W\left(\mathbf{x}_{\mathcal{B} \backslash \mathcal{S}}\right), W\left(\mathbf{x}_{\mathcal{S}}\right)$ are the weights corresponding to the injection points in Table 4.2.1-1.

\footnotetext{
${ }^{26}$ which renders not trivial the time integration of $\dot{\boldsymbol{R}}_{\text {mech }}(t)$.
} 


\section{A.1.1 Rephrased numerical quadrature at the CSM injection domain $\mathcal{B}_{\text {loc }}(t)$}

The term in equation A.(2)-(b) can be rephrased as:

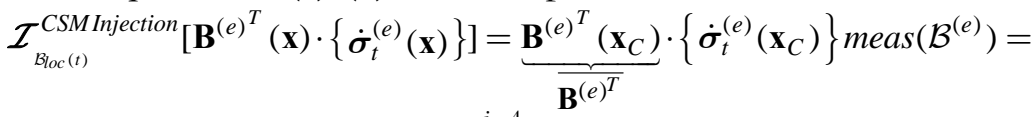

$$
\begin{aligned}
& =\underbrace{\overline{\mathbf{s}^{(e)^{T}}}}_{\sum_{i=1}^{i=4} \mathbf{B}^{(e)^{T}}\left(\mathbf{x}_{i}\right) \cdot w_{i}\left(\mathbf{x}_{i}\right)} \operatorname{meas}\left(\mathcal{B}^{(e)}\right) \cdot\left\{\dot{\boldsymbol{\sigma}}_{t}^{(e)}\left(\mathbf{x}_{C}\right)\right\}=\sum_{i=1}^{i=4} \mathbf{B}^{(e)^{T}}\left(\mathbf{x}_{i}\right) \cdot\left\{\dot{\boldsymbol{\sigma}}_{t}^{(e)}\left(\mathbf{x}_{C}\right)\right\} w_{i}\left(\mathbf{x}_{i}\right)= \\
& =\mathcal{I}_{\text {Bloc }(t)}^{\text {Gauss }}[\mathbf{B}^{(e)^{T}}(\mathbf{x}) \cdot \underbrace{\left\{\dot{\tilde{\sigma}}_{i}^{(e)}(\mathbf{x})\right\}}_{\begin{array}{c}
\text { constant } \\
\text { at all }
\end{array}}] \\
& \dot{\tilde{\sigma}}_{t}^{(e)}\left(\mathbf{x}_{i}\right)=\dot{\boldsymbol{\sigma}}_{t}^{(e)}\left(\mathbf{x}_{C}\right)=\dot{\bar{\sigma}}_{t}^{(e)}, i=(1,2,3,4)
\end{aligned}
$$

where the smooth character of the deformation matrix, $\mathbf{B}^{(e)}(\mathbf{x})$ and the mean value theorem has been considered.

Results in equation A.(3) involve the definition of the equivalent stresses computed at the standard Gauss points, $\tilde{\boldsymbol{\sigma}}_{t}^{(e)}\left(\mathbf{x}_{i}\right)$, defined trough

$$
\dot{\tilde{\sigma}}_{t}^{(e)}\left(\mathbf{x}_{i}\right)=\dot{\overline{\boldsymbol{\sigma}}}_{t}^{(e)} \quad, i=(1,2,3,4), \forall \mathcal{B}^{(e)} \subset \mathcal{B}_{l o c}(t)
$$

and fulfilling the following property:

$$
\mathcal{I}_{\mathcal{B}_{\text {loc }}^{\text {CSM }(t)}}^{\text {Cijjection }}\left[\mathbf{B}^{(e)^{T}}(\mathbf{x}) \cdot\left\{\dot{\boldsymbol{\sigma}}_{t}^{(e)}(\mathbf{x})\right\}\right]=\mathcal{I}_{\mathcal{B}_{\text {loc }}^{\text {Gauss }}}^{\text {Ge }}\left[\mathbf{B}^{(e)^{T}}(\mathbf{x}) \cdot\left\{\dot{\tilde{\boldsymbol{\sigma}}}_{t}^{(e)}(\mathbf{x})\right\}\right]
$$

\section{A.1.2 Rephrased numerical quadrature at the DDM injection domain $\mathcal{B}_{\text {dis }}(t)$}

In turn, the term in equation A.(2)-(c) can be rephrased as:

$$
\begin{aligned}
& \mathcal{I}_{\text {Bdis }^{\text {DIt }}(t)}^{\text {DDMijection }}\left[\mathbf{B}^{(e)^{T}}(\mathbf{x}) \cdot\left\{\dot{\boldsymbol{\sigma}}_{t}^{(e)}(\mathbf{x})\right\}\right]=\underbrace{\mathbf{B}^{(e)^{T}}\left(\mathbf{x}_{\mathcal{B} \backslash \mathcal{S}}\right)}_{=\mathbf{B}^{(e)^{T}}\left(\mathbf{x}_{\mathcal{S}}\right)} \cdot\left\{\dot{\boldsymbol{\sigma}}_{t}^{(e)}\left(\mathbf{x}_{\mathcal{B} \backslash \mathcal{S}}\right)\right\} W\left(\mathbf{x}_{\mathcal{B} \backslash \mathcal{S}}\right)+ \\
& +\mathbf{B}^{()^{T}}\left(\mathbf{x}_{\mathcal{S}}\right) \cdot\left\{\dot{\boldsymbol{\sigma}}_{t}^{(e)}\left(\mathbf{x}_{\mathcal{S}}\right)\right\} W\left(\mathbf{x}_{\mathcal{S}}\right)
\end{aligned}
$$

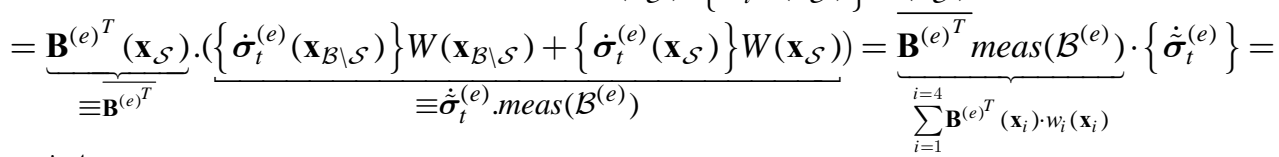

$$
\begin{aligned}
& =\sum_{i=1}^{i=4} \mathbf{B}^{(e)^{T}}\left(\mathbf{x}_{i}\right) \cdot \underbrace{\left\{\dot{\tilde{\boldsymbol{\sigma}}}_{t}^{(e)}(\mathbf{x})\right\}}_{\begin{array}{c}
\text { constant } \\
\text { atall } \mathbf{x}_{i}
\end{array}} w_{i}\left(\mathbf{x}_{i}\right)=\mathcal{I}_{\mathcal{B}_{\text {dis }}(t)}^{\text {Gauss }}\left[\mathbf{B}^{(e)^{T}} \cdot \dot{\tilde{\boldsymbol{\sigma}}}_{t}^{(e)}(\mathbf{x})\right] \\
& \left\{\begin{array}{l}
\dot{\boldsymbol{\sigma}}_{t}^{(e)}\left(\mathbf{x}_{i}\right) \equiv \frac{1}{\operatorname{meas}\left(\mathcal{B}^{(e)}\right)}\left[\dot{\boldsymbol{\sigma}}_{t}^{(e)}\left(\mathbf{x}_{\mathcal{B} \backslash \mathcal{S}}\right) W\left(\mathbf{x}_{\mathcal{B} \backslash \mathcal{S}}\right)+\dot{\boldsymbol{\sigma}}_{t}^{(e)}\left(\mathbf{x}_{\mathcal{S}}\right) W\left(\mathbf{x}_{\mathcal{S}}\right)\right]= \\
=\underbrace{\left(1-k^{(e)} \cdot \frac{\operatorname{meas}\left(\mathcal{S}^{(e)}\right)}{\operatorname{meas}\left(\mathcal{B}^{(e)}\right)}\right)}_{(1-\xi)} \cdot \dot{\boldsymbol{\sigma}}_{t}^{(e)}\left(\mathbf{x}_{\mathcal{B} \backslash \mathcal{S}}\right)+\underbrace{k^{(e)} \cdot \frac{\operatorname{meas}\left(\mathcal{S}^{(e)}\right)}{\operatorname{meas}\left(\mathcal{B}^{(e)}\right)}}_{\xi} \dot{\boldsymbol{\sigma}}_{t}^{(e)}\left(\mathbf{x}_{\mathcal{S}}\right)=\quad, i=(1,2,3,4) \\
=\xi \dot{\boldsymbol{\sigma}}_{t}^{(e)}\left(\mathbf{x}_{\mathcal{S}}\right)+(1-\xi) \dot{\boldsymbol{\sigma}}_{t}^{(e)}\left(\mathbf{x}_{\mathcal{B} \backslash \mathcal{S}}\right)
\end{array}\right. \\
& k^{(e)} \cdot \frac{\operatorname{meas}\left(\mathcal{S}^{(e)}\right)}{\operatorname{meas}\left(\mathcal{B}^{(e)}\right)}=\frac{k^{(e)}}{\ell^{(e)}}=\xi
\end{aligned}
$$

where, again, the smooth character of the deformation matrix, $\left(\mathbf{B}^{(e)}\left(\mathbf{x}_{\mathcal{B} \backslash \mathcal{S}}\right)=\mathbf{B}^{(e)}\left(\mathbf{x}_{\mathcal{S}}\right) \equiv \overline{\mathbf{B}^{(e)}}\right)$ has been considered. In equation A.(6), $\ell^{(e)}$ is the finite element characteristic length computed according (Oliver 1989) (see equation (60)) and $\xi$ is a user defined parameter used for regularization purposes (see equation (61)).

Results in equation A.(6) involve the definition of some new equivalent stresses computed at the standard Gauss points, $\tilde{\sigma}_{t}^{(e)}\left(\mathbf{x}_{i}\right)$, through:

$$
\dot{\tilde{\sigma}}_{t}^{(e)}\left(\mathbf{x}_{i}\right) \equiv(1-\xi) \dot{\boldsymbol{\sigma}}_{t}^{(e)}\left(\mathbf{x}_{\mathcal{B} \backslash \mathcal{S}}\right)+\xi \dot{\boldsymbol{\sigma}}_{t}^{(e)}\left(\mathbf{x}_{\mathcal{S}}\right), \quad i=(1,2,3,4), \forall \mathcal{B}^{(e)} \subset \mathcal{B}_{\text {dis }}(t)
$$


fulfilling the following property:

$$
\mathcal{I}_{\mathcal{B}_{\text {dis }}^{\text {DD }(t)}}^{\text {injection }}\left[\mathbf{B}^{(e)^{T}}(\mathbf{x}) \cdot\left\{\dot{\boldsymbol{\sigma}}_{t}^{(e)}(\mathbf{x})\right\}\right]=\mathcal{I}_{\mathcal{B}_{\text {dis }}^{\text {Gauss }}}^{\text {at }}\left[\mathbf{B}^{(e)^{T}}(\mathbf{x}) \cdot\left\{\dot{\tilde{\boldsymbol{\sigma}}}_{t}^{(e)}(\mathbf{x})\right\}\right]
$$

\section{A.1.3 Rephrased numerical quadrature at the remaining domain $\mathcal{B} \backslash \mathcal{B}_{\text {inj }}(t)$}

The term in equation A.(2)-(a) is already a four-point Gauss quadrature. Therefore, the following equivalence is straightforward:

$$
\dot{\tilde{\sigma}}_{t}^{(e)}\left(\mathbf{x}_{i}\right)=\dot{\tilde{\sigma}}_{t}^{(e)}\left(\mathbf{x}_{i}\right) \quad, i=(1,2,3,4), \forall \mathcal{B}^{(e)} \subset \mathcal{B} \backslash \mathcal{B}_{\text {inj }}(t)
$$

fulfilling the following property:

$$
\mathcal{I}_{B \backslash B \text { inj }(t)}^{\text {Gauss }}\left[\mathbf{B}^{(e)^{T}}(\mathbf{x}) \cdot\left\{\dot{\boldsymbol{\sigma}}_{t}^{(e)}(\mathbf{x})\right\}\right]=\mathcal{I}_{B \backslash B \text { inj }(t)}^{\text {Gauss }}\left[\mathbf{B}^{(e)^{T}}(\mathbf{x}) \cdot\left\{\dot{\tilde{\boldsymbol{\sigma}}}_{t}^{(e)}(\mathbf{x})\right\}\right]
$$

\section{A.1.4 Unified numerical quadrature at the total domain $\mathcal{B}=\left(\mathcal{B} \backslash \mathcal{B}_{\text {inj }}(t)\right) \cup \mathcal{B}_{\text {loc }}(t) \cup \mathcal{B}_{\text {dis }}(t)$}

Results in equation A.(5), A.(8) and A.(10) can be inserted into equation A.(1) yielding:

$$
\begin{aligned}
& \dot{\boldsymbol{R}}_{\text {mech }}\left(\dot{\hat{\boldsymbol{u}}}^{h}(t), \mathcal{B} \backslash \mathcal{B}_{\text {inj }}(t), \mathcal{B}_{\text {loc }}(t), \mathcal{B}_{\text {dis }}(t)\right)= \\
& =\sum_{\forall \mathcal{B}^{(e)} \subset \mathcal{B} \backslash \mathcal{B}_{i j j}(t)} \mathcal{I}_{\mathcal{B B i n s i j}^{\text {Git }}}^{\text {Gaus }}\left[\mathbf{B}^{(e)^{T}}(\mathbf{x}) \cdot\left\{\dot{\tilde{\boldsymbol{\sigma}}}_{t}^{(e)}(\mathbf{x})\right\}\right]+ \\
& +\sum_{\forall \mathcal{B}^{(e)} \subset \mathcal{B}_{\text {loc }}(t)} \mathcal{I}_{\mathcal{B}_{\text {loc }}(t)}^{\text {Gauss }}\left[\mathbf{B}^{(e)^{T}}(\mathbf{x}) \cdot\left\{\dot{\boldsymbol{\sigma}}_{t}^{(e)}(\mathbf{x})\right\}\right]+ \\
& +\sum_{\forall \mathcal{B}^{(e)} \subset \mathcal{B}_{\text {dis }}(t)} \mathcal{I}_{\mathcal{B}_{\text {dis }}(t)}^{\text {Gauss }}\left[\mathbf{B}^{(e)^{T}}(\mathbf{x}) \cdot\left\{\dot{\boldsymbol{\sigma}}_{t}^{(e)}(\mathbf{x})\right\}\right]-\dot{\mathbf{F}}^{\text {ext }}
\end{aligned}
$$

Where the equivalent stresses $\tilde{\sigma}_{t}^{(e)}\left(\mathbf{x}_{i}\right), i=(1,2,3,4)$ are computed according the corresponding injection domains (see equations A.(4), A.(7) and A.(9)), i.e:

$$
\begin{array}{lrl}
\dot{\tilde{\sigma}}_{t}^{(e)}\left(\mathbf{x}_{i}\right)=\dot{\boldsymbol{\sigma}}_{t}^{(e)}\left(\mathbf{x}_{i}\right)=\tau^{(e)}(t) \dot{\boldsymbol{\sigma}}_{t}^{(e)}\left(\mathbf{x}_{i}\right)+\left(1-\tau^{(e)}(t)\right) \dot{\dot{\sigma}}_{t}^{(e)} & , \mathcal{B}^{(e)} \subset \mathcal{B} \backslash \mathcal{B}_{i n j}(t) \\
\dot{\tilde{\boldsymbol{\sigma}}}_{t}^{(e)}\left(\mathbf{x}_{i}\right)=\dot{\overline{\boldsymbol{\sigma}}}_{t}^{(e)} & , \mathcal{B}^{(e)} \subset \mathcal{B}_{\text {loc }}(t) \\
\dot{\tilde{\sigma}}_{t}^{(e)}\left(\mathbf{x}_{i}\right) \equiv(1-\xi) \dot{\boldsymbol{\sigma}}_{t}^{(e)}\left(\mathbf{x}_{\mathcal{B} \backslash \mathcal{S}}\right)+\xi \dot{\boldsymbol{\sigma}}_{t}^{(e)}\left(\mathbf{x}_{\mathcal{S}}\right) & , \mathcal{B}^{(e)} \subset \mathcal{B}_{\text {dis }}(t)
\end{array}
$$

Therefore, they can be interpreted as some effective stresses computed for integration proposes at all Gauss-integration points of the domain $\mathcal{B}$. Finally, expression A.(11) can be trivially condensed in terms of the equivalent/effective stresses $\tilde{\boldsymbol{\sigma}}_{n+1}^{(e)}\left(\mathbf{x}_{i}\right)$ in equation A.(12) yielding:

$$
\begin{aligned}
& \dot{\boldsymbol{R}}_{\text {mech }}\left(\dot{\tilde{\boldsymbol{\sigma}}}\left(\hat{\boldsymbol{u}}^{h}\left(t_{n+1}\right)\right)=\right. \\
& =\underbrace{\sum_{\mathcal{B}^{(e)} \subset \mathcal{B}} \mathcal{I}_{\mathcal{B}}^{\text {Gauss }}\left[\mathbf{B}^{(e)^{T}}(\mathbf{x}) \cdot\left\{\dot{\tilde{\boldsymbol{\sigma}}}_{t}^{(e)}(\mathbf{x})\right\}\right]}_{\approx \int_{\mathcal{B}} \mathbf{B}^{T} \cdot\left\{\dot{\boldsymbol{\sigma}}_{t}(\mathbf{x})\right\} d \mathcal{B}}-\dot{\mathbf{F}}^{\text {ext }}(t)=\dot{\boldsymbol{R}}_{\text {mech }}\left(\dot{\tilde{\boldsymbol{\sigma}}}_{t}\right)
\end{aligned}
$$

where condition $\mathcal{B}=\left(\mathcal{B} \backslash \mathcal{B}_{\text {inj }}(t)\right) \cup \mathcal{B}_{\text {loc }}(t) \cup \mathcal{B}_{\text {dis }}(t)$ has also been considered. It is worth nothing that, the use of the effective stresses definition in equation A.(12) allows removing the direct dependence of the residue rate, $\dot{\boldsymbol{R}}_{\text {mech }}\left(\dot{\tilde{\sigma}}_{t}\right)$ on the injection domains. Therefore, the residue in equation A.(13), now considered a functional of the effective stresses, $\dot{\tilde{\sigma}}_{t}$, can be trivially integrated in time, considering the initial conditions $\left.\tilde{\boldsymbol{\sigma}}_{t}^{(e)}(\mathbf{x})\right|_{t=0}=\mathbf{0}$, yielding,

$$
\begin{aligned}
& \boldsymbol{R}_{\text {mech }}(\tilde{\boldsymbol{\sigma}}\left(\hat{\boldsymbol{u}}^{h}\left(t_{n+1}\right)\right)=\sum_{\forall \mathcal{B}^{(e)} \subset \mathcal{B}} \underbrace{\mathcal{I}_{\text {Gauss }}\left[\mathbf{B}^{(e)^{T}}(\mathbf{x}) \cdot\left\{\tilde{\boldsymbol{\sigma}}^{(e)}(\mathbf{x})\right\}\right]}_{\approx \int_{\mathcal{B}^{(e)}} \mathbf{B}^{(e)^{T}}(\mathbf{x}) \cdot\left\{\tilde{\boldsymbol{\sigma}}_{n+1}^{(e)}(\mathbf{x})\right\} d \mathcal{B}}-\mathbf{F}_{n+1}^{e x t}= \\
& =\sum_{\forall \mathcal{B}^{(e)} \subset \mathcal{B}} \int_{\mathcal{B}^{(e)}} \mathbf{B}^{(e)^{T}}(\mathbf{x}) \cdot\left\{\tilde{\boldsymbol{\sigma}}_{n+1}^{(e)}(\mathbf{x})\right\} d \mathcal{B}-\mathbf{F}_{n+1}^{e x t}
\end{aligned}
$$


where the effective stresses, $\tilde{\boldsymbol{\sigma}}_{n+1}^{(e)}(\mathbf{x})$, are incrementally computed through time integration of equations A.(12) i.e.,

$$
\left\{\begin{aligned}
\tilde{\boldsymbol{\sigma}}_{n+1}^{(e)}\left(\mathbf{x}_{i}\right)=\tilde{\boldsymbol{\sigma}}_{n}^{(e)}\left(\mathbf{x}_{i}\right)+\Delta \tilde{\boldsymbol{\sigma}}_{n+1}^{(e)}\left(\mathbf{x}_{i}\right) & i=(1,2,3,4)
\end{aligned}\right.
$$

which matches the definitions BOX 4.2-2. Finally, the total mechanics residue in equation (69) can be computed using a standard Gauss- quadrature in terms of the effective stresses in equation A.(15), as

$$
\left\{\begin{array}{l}
\boldsymbol{R}_{\text {mech }}\left(\dot{\tilde{\boldsymbol{\sigma}}}\left(\hat{\boldsymbol{u}}^{h}\left(t_{n+1}\right)\right)=\sum_{\forall \mathcal{B}^{(e)} \subset \mathcal{B}} \mathcal{I}_{\mathcal{B}}^{\text {Gauss }}\left[\mathbf{B}^{(e)^{T}}(\mathbf{x}) \cdot\left\{\tilde{\boldsymbol{\sigma}}_{n+1}^{(e)}(\mathbf{x})\right\}\right]-\mathbf{F}_{\text {ext }}\right. \\
\mathcal{I}_{\mathcal{B}}^{\text {Gauss }}\left[\mathbf{B}^{(e)^{T}}(\mathbf{x}) \cdot\left\{\tilde{\boldsymbol{\sigma}}_{n+1}^{(e)}(\mathbf{x})\right\}\right]=\sum_{i=1}^{i=4} \mathbf{B}^{(e)^{T}}\left(\mathbf{x}_{i}\right) \cdot\left\{\tilde{\boldsymbol{\sigma}}_{n+1}^{(e)}\left(\mathbf{x}_{i}\right)\right\} w_{i}\left(\mathbf{x}_{i}\right)
\end{array}\right.
$$

\section{A.2 Strain injection. Expressions for implementation}

For the elements injected with a DDM, the element-wise constant degrees of freedom $\llbracket \dot{\boldsymbol{u}} \rrbracket^{(e)}$ (that have elemental support), can be solved, at the element level, by a standard condensation procedure that is summarized here.

The residual balance of forces $\dot{\boldsymbol{R}}^{(e)} \equiv \dot{\boldsymbol{R}}_{\text {mech }}$ in equation A.(13), and the residue of equation (65)-(b), $\dot{r}^{(e)}$, can be expressed in the following form:

$$
-\left\{\begin{array}{c}
\dot{\boldsymbol{R}}^{(e)} \\
\dot{\boldsymbol{r}}^{(e)}
\end{array}\right\}=\left[\begin{array}{cc}
\boldsymbol{K}_{u \boldsymbol{u}}^{(e)} & \boldsymbol{K}_{u \llbracket \boldsymbol{u} \rrbracket}^{(e)} \\
\boldsymbol{K}_{\llbracket \boldsymbol{u} \rrbracket u}^{(e)} & \boldsymbol{K}_{\llbracket \boldsymbol{u} \rrbracket \llbracket u \rrbracket}^{(e)}
\end{array}\right]\left\{\begin{array}{c}
\{\dot{\boldsymbol{u}}\}^{(e)} \\
\llbracket \dot{\boldsymbol{u}} \rrbracket^{(e)}
\end{array}\right\} \quad, \mathcal{B}^{(e)} \subset \mathcal{B}_{\text {dis }}
$$

where $\{\dot{\boldsymbol{u}}\}^{(e)}$ stands for the element vector $\{\dot{\boldsymbol{u}}\}^{(e)}=\left\{\dot{\boldsymbol{u}}_{1}^{T}, \dot{\boldsymbol{u}}_{2}^{T}, \dot{\boldsymbol{u}}_{3}^{T}, \dot{\boldsymbol{u}}_{4}^{T}\right\}^{T}$ composed by the $\dot{\boldsymbol{u}}_{i}=\left\{\dot{u}_{x}, \dot{u}_{y}\right\}_{i}^{T}$ degrees of freedom, and $\boldsymbol{K}_{u u}^{(e)}=\frac{\partial \dot{\boldsymbol{R}}^{(e)}}{\partial \dot{\boldsymbol{u}}^{(e)}}, \boldsymbol{K}_{u \llbracket \boldsymbol{u} \rrbracket}^{(e)}=\frac{\partial \dot{\boldsymbol{R}}^{(e)}}{\partial \llbracket \dot{\boldsymbol{u}} \rrbracket^{(e)}}, \mathbf{K}_{\llbracket \boldsymbol{u} \rrbracket u}^{(e)}=\frac{\partial \dot{\boldsymbol{r}}^{(e)}}{\partial \dot{\boldsymbol{u}}^{(e)}}$ and $\boldsymbol{K}_{\llbracket u \rrbracket \llbracket}^{(e)}=\frac{\partial \dot{\boldsymbol{r}}^{(e)}}{\partial \llbracket \dot{\boldsymbol{u}} \rrbracket^{(e)}}$ are the elemental tangent stiffness matrixes.

Solving the second equation of A.(17) for $\llbracket \dot{\boldsymbol{u}} \rrbracket^{(e)}$ :

$$
\llbracket \dot{\boldsymbol{u}} \rrbracket^{(e)}=-\left(\boldsymbol{K}_{\llbracket u \llbracket \llbracket u \rrbracket}^{(e)}\right)^{-1}\left(\dot{\boldsymbol{r}}^{(e)}+\mathbf{K}_{\llbracket u \rrbracket u}^{(e)}\{\dot{\boldsymbol{u}}\}^{(e)}\right) \quad, \mathcal{B}^{(e)} \subset \mathcal{B}_{\text {dis }}
$$

by substituting $\llbracket \dot{\boldsymbol{u}} \rrbracket^{(e)}$ in the first equation of A.(17) and reorganizing terms:

$$
-\underbrace{\left(\dot{\boldsymbol{R}}^{(e)}-\boldsymbol{K}_{u \llbracket u \rrbracket}^{(e)}\left(\boldsymbol{K}_{\llbracket u \llbracket u \rrbracket}^{(e)}\right)^{-1} \dot{r}^{(e)}\right)}_{\dot{\hat{\boldsymbol{R}}}^{(e)}}=\underbrace{\left(\boldsymbol{K}_{u u}^{(e)}-\left(\boldsymbol{K}_{u \llbracket u \rrbracket}^{(e)}\left(\boldsymbol{K}_{\llbracket u \llbracket u \rrbracket}^{(e)}\right)^{-1} \boldsymbol{K}_{\llbracket u \rrbracket u}^{(e)}\right)\right)}_{\hat{\boldsymbol{K}}^{(e)}}\{\dot{\boldsymbol{u}}\}^{(e)}, \mathcal{B}^{(e)} \subset \mathcal{B}_{d i s}
$$

where $\hat{\boldsymbol{K}}^{(e)}$ and $\dot{\hat{\boldsymbol{R}}}^{(e)}$ are the elemental condensed stiffness matrix and residual vector respectively ${ }^{27}$ :

${ }^{27}$ For the elements, $\mathcal{B}^{(e)} \subset \mathcal{B} \backslash \mathcal{B}_{\text {dis }}$, where no condensation procedure is necessary, the elemental residue vectors and tangent stiffness matrixes can be inserted in the general framework by A.(20) -(b). 


$$
\begin{aligned}
& \left\{\begin{array}{l}
\dot{\hat{\boldsymbol{R}}}^{(e)}=\dot{\boldsymbol{R}}^{(e)}-\boldsymbol{K}_{u \llbracket u \rrbracket}^{(e)}\left(\boldsymbol{K}_{\llbracket u \rrbracket u \rrbracket}^{(e)}\right)^{-1} \dot{\boldsymbol{r}}^{(e)} \\
\hat{\boldsymbol{K}}^{(e)}=\boldsymbol{K}_{u u}^{(e)}-\left(\boldsymbol{K}_{u \llbracket u \rrbracket}^{(e)}\left(\boldsymbol{K}_{\llbracket u \rrbracket \llbracket u \rrbracket}^{(e)}\right)^{-1} \boldsymbol{K}_{\llbracket u \rrbracket u}^{(e)}\right)
\end{array}\right. \\
& \begin{cases}\dot{\hat{\boldsymbol{R}}}^{(e)}=\dot{\boldsymbol{R}}^{(e)} & , \mathcal{B}^{(e)} \subset \mathcal{B}_{\text {dis }} \\
\hat{\boldsymbol{K}}^{(e)}=\boldsymbol{K}_{u u}^{(e)} & \mathcal{B}^{(e)} \subset \mathcal{B} \backslash \mathcal{B}_{d i s}\end{cases}
\end{aligned}
$$

Once the condensed element matrices are computed, the global entities $\hat{\boldsymbol{K}}$ and $\dot{\hat{\boldsymbol{R}}}$ can be assembled and then $\{\dot{\boldsymbol{u}}\}$ can be solved at the global level in a standard manner:

$$
-\dot{\hat{\boldsymbol{R}}}=\hat{\boldsymbol{K}} \cdot\{\dot{\boldsymbol{u}}\}
$$

where :

$$
\hat{\boldsymbol{K}}=\underset{e \in \mathcal{B}_{\text {dis }}}{\mathcal{A}}\left(\hat{\boldsymbol{K}}^{(e)}\right), \dot{\hat{\boldsymbol{R}}}=\underset{e \in \mathcal{B}_{\text {sis }}}{\mathcal{A}}\left(\dot{\hat{\boldsymbol{R}}}^{(e)}\right)
$$

where $\{\dot{\boldsymbol{u}}\}$ stands for the global vector composed by the $\dot{\boldsymbol{u}}_{i}$ degrees of freedom $\{\dot{\boldsymbol{u}}\}=\left\{\dot{\boldsymbol{u}}_{1}, \dot{\boldsymbol{u}}_{2,}, \ldots, \dot{\boldsymbol{u}}_{\text {nnodes }}\right\}^{T}$, being nnodes the total number of nodes of the finite element mesh).

Once equation A.(21) is solved for $\{\dot{\boldsymbol{u}}\}, \llbracket \dot{\boldsymbol{u}} \rrbracket^{(e)}$ can be computed at the element level, by using expression A.(18), in those elements belonging to $\mathcal{B}_{\text {dis }}$.

Expressions for the entities in equation A.(20) are summarized in BOX A-1.

$$
\begin{aligned}
& \text { BOX A-1 - DDM injection - expressions for implementation } \\
& \boldsymbol{R}^{(e)}=\sum_{i=1}^{i=4} \mathbf{B}^{(e)^{T}}\left(\mathbf{x}_{i}\right) \cdot\left\{\tilde{\boldsymbol{\sigma}}_{n+1}^{(e)}\left(\mathbf{x}_{i}\right)\right\} \cdot w\left(\mathbf{x}_{i}\right)-\mathbf{F}_{n+1}^{e x t}(e) \\
& \boldsymbol{r}^{(e)}=\operatorname{meas}\left(\mathcal{S}^{(e)}\right) \cdot\left[\mathbf{n}^{(e)}\right] \cdot\left(\left\{\boldsymbol{\sigma}_{n+1}^{(e)}\left(\mathbf{x}_{\mathcal{S}}\right)\right\}-\left\{\boldsymbol{\sigma}_{n+1}^{(e)}\left(\mathbf{x}_{\mathcal{B} \backslash \mathcal{S}}\right)\right\}\right) \quad \forall \mathcal{B}^{(e)} \in \mathcal{B}_{\text {dis }}\left(t_{n+1}\right) \\
& \left\{\boldsymbol{K}_{u u}^{(e)}=\tau_{n+1}^{(e)} \cdot \sum_{i=1}^{i=4} \mathbf{B}^{(e)^{T}}\left(\mathbf{x}_{i}\right) \cdot\left\{\mathcal{C}_{n+1}^{(e)}\left(\mathbf{x}_{i}\right)\right\} \cdot \mathbf{B}^{(e)}\left(\mathbf{x}_{i}\right) \cdot w\left(\mathbf{x}_{i}\right)+\right. \\
& +\left(1-\tau_{n+1}^{(e)}\right) \cdot \mathbf{B}^{(e)^{T}}\left(\mathbf{x}_{\mathcal{S}}\right) \cdot\left\{\mathcal{C}_{n+1}^{(e)}\left(\mathbf{x}_{\mathcal{S}}\right)\right\} \cdot \mathbf{B}^{(e)}\left(\mathbf{x}_{\mathcal{S}}\right) \cdot \operatorname{meas}\left(\mathcal{B}^{(e)}\right) \\
& \boldsymbol{K}_{u u}^{(e)}=\mathbf{B}^{(e)^{T}}\left(\mathbf{x}_{\mathcal{S}}\right) \cdot\left\{\mathcal{C}_{n+1}^{(e)}\left(\mathbf{x}_{\mathcal{S}}\right)\right\} \cdot \mathbf{B}^{(e)}\left(\mathbf{x}_{\mathcal{S}}\right) \cdot \operatorname{meas}\left(\mathcal{B}^{(e)}\right) \quad, \mathcal{B}^{(e)} \subset \mathcal{B}_{l o c} \\
& \left\{\boldsymbol{K}_{u u}^{(e)}=\mathbf{B}^{(e)^{T}}\left(\mathbf{x}_{\mathcal{S}}\right) \cdot\left\{\mathcal{C}_{n+1}^{(e)}\left(\mathbf{x}_{\mathcal{S}}\right)\right\} \cdot \mathbf{B}^{(e)}\left(\mathbf{x}_{\mathcal{S}}\right) \cdot W\left(\mathbf{x}_{\mathcal{S}}\right)+\right. \\
& +\mathbf{B}^{(e)^{T}}\left(\mathbf{x}_{\mathcal{B} \backslash \mathcal{S}}\right) \cdot\left\{\mathcal{C}_{n+1}^{(e)}\left(\mathbf{x}_{\mathcal{B} \backslash \mathcal{S}}\right)\right\} \cdot \mathbf{B}^{(e)}\left(\mathbf{x}_{\mathcal{B} \backslash \mathcal{S}}\right) \cdot W\left(\mathbf{x}_{\mathcal{B} \backslash \mathcal{S}}\right) \\
& \boldsymbol{K}_{u \llbracket u \rrbracket}^{(e)}=\mathbf{B}^{(e)^{T}}\left(\mathbf{x}_{\mathcal{S}}\right) \cdot\left\{\mathcal{C}_{n+1}^{(e)}\left(\mathbf{x}_{\mathcal{S}}\right)\right\} \cdot\left(\frac{1}{k_{n+1}^{(e)}}\left[\mathbf{n}^{(e)}\right]-\left[\nabla \varphi_{n+1}^{(e)}\left(\mathbf{x}_{\mathcal{S}}\right)\right]\right) \cdot W\left(\mathbf{x}_{\mathcal{S}}\right)- \\
& -\mathbf{B}^{(e)^{T}}\left(\mathbf{x}_{\mathcal{B} \backslash \mathcal{S}}\right) \cdot\left\{\mathcal{C}_{n+1}^{(e)}\left(\mathbf{x}_{\mathcal{B} \backslash \mathcal{S}}\right)\right\} \cdot\left[\nabla \varphi_{n+1}^{(e)}\left(\mathbf{x}_{\mathcal{B} \backslash \mathcal{S}}\right)\right] \cdot W\left(\mathbf{x}_{\mathcal{B} \backslash \mathcal{S}}\right) \\
& \boldsymbol{K}_{\llbracket u \rrbracket u}^{(e)}=\operatorname{meas}\left(\mathcal{S}^{(e)}\right) \cdot\left[\mathbf{n}^{(e)}\right] \cdot\left\{\mathcal{C}_{n+1}^{(e)}\left(\mathbf{x}_{\mathcal{S}}\right)\right\} \cdot \mathbf{B}^{(e)}\left(\mathbf{x}_{\mathcal{S}}\right) \\
& -\operatorname{meas}\left(\mathcal{S}^{(e)}\right) \cdot\left[\mathbf{n}^{(e)}\right] \cdot\left\{\mathcal{C}_{n+1}^{(e)}\left(\mathbf{x}_{\mathcal{B} \backslash \mathcal{S}}\right)\right\} \cdot \mathbf{B}^{(e)}\left(\mathbf{x}_{\mathcal{B} \backslash \mathcal{S}}\right) \\
& \boldsymbol{K}_{\llbracket u \rrbracket u}^{(e)}=\operatorname{meas}\left(\mathcal{S}^{(e)}\right)\left[\mathbf{n}^{(e)}\right] \cdot\left\{\mathcal{C}_{n+1}^{(e)}\left(\mathbf{x}_{\mathcal{S}}\right)\right\} \cdot\left(\frac{1}{k_{n+1}^{(e)}}\left[\mathbf{n}^{(e)}\right]-\left[\nabla \varphi_{n+1}^{(e)}\left(\mathbf{x}_{\mathcal{S}}\right)\right]\right)+ \\
& +\operatorname{meas}\left(\mathcal{S}^{(e)}\right)\left[\mathbf{n}^{(e)}\right] \cdot\left\{\mathcal{C}_{n+1}^{(e)}\left(\mathbf{x}_{\mathcal{B} \backslash \mathcal{S}}\right)\right\} \cdot\left[\nabla \varphi_{n+1}^{(e)}\left(\mathbf{x}_{\mathcal{B} \backslash \mathcal{S}}\right)\right] \\
& \tau_{n+1}^{(e)}=\tau_{\text {stab }} \quad \forall \mathcal{B}^{(e)} \rightarrow\left\{\begin{array}{l}
\tau_{\text {stab }}=0.1 \rightarrow \text { isochoric case } \\
\tau_{\text {stab }}=1.0 \rightarrow \text { general case }
\end{array}\right. \\
& , \mathcal{B}^{(e)} \subset \mathcal{B} \backslash \mathcal{B}_{\text {dis }} \\
& , \mathcal{B}^{(e)} \subset \mathcal{B}_{\text {dis }}
\end{aligned}
$$


where $w\left(\mathbf{x}_{i}\right), i=(1,2,3,4)$ are the weights corresponding to the four-point Gauss integration, $W\left(\mathbf{x}_{\mathcal{B} \backslash \mathcal{S}}\right)$ and $W\left(\mathbf{x}_{\mathcal{S}}\right)$ are the weights corresponding to the injection points in Table 4.2.1-1, $\tilde{\boldsymbol{\sigma}}_{n+1}^{(e)}(\mathbf{x})$ are the effective stresses computed from BOX 4.2-2, $\mathcal{C}_{n+1}^{(e)}(\mathbf{x})$ stands for the in-loading tangent constitutive operator, $\mathbf{F}_{n+1}^{\text {ext }}(e)$ stands for elemental external forces, computed in a regular manner and meas $\left(\mathcal{S}^{(e)}\right)$ is computed from equation $(60)$ as:

$$
\operatorname{meas}\left(\mathcal{S}^{(e)}\right)=\frac{\operatorname{meas}\left(\mathcal{B}^{(e)}\right)}{\ell^{(e)}}
$$

being $\ell^{(e)}$ the finite element characteristic length (computed according (Oliver 1989)).

\section{A.3 -Discontinuous displacement mode (DDM) injection. Selection of the unit normal vector}

Determination of the injection normal $\mathbf{n}^{(e)}$, to be used in the DDM injection procedure in BOX A-1, is a relevant aspect of the proposed methodology. After computing the crackpath-field , $\boldsymbol{\mu}$, the explicit position of the elemental crack path $\Gamma^{(e)}$, and its corresponding unit normal $\mathbf{n}_{\Gamma}^{(e)}$, can be straightforwardly computed at the element level in terms of the position of the zero value of $\boldsymbol{\mu}$ along the element sides (see Figure A. 1). Then, two options for the determination of $\mathbf{n}^{(e)}$ arise:

OPTION I: Crack path field criterion. The normal is evaluated directly as that of the elemental crack path $\left(\mathbf{n}^{(e)}=\mathbf{n}_{T}^{(e)}\right)$

OPTION II: Bifurcation analysis criterion: $\mathbf{n}^{(e)}$ is the one out of the two solutions $\left(\mathbf{n}_{1}^{(e)}, \mathbf{n}_{2}^{(e)}\right)$ obtained from the discontinuous bifurcation analysis (performed at the bifurcation time $t_{B}^{(e)}$ and stored at that time), whose direction approaches better the elemental crack path normal, $\mathbf{n}_{\Gamma}^{(e)}$. This evaluation is done in terms of the absolute value of the dot product of every of the two vectors times $\mathbf{n}_{\Gamma}^{(e)}$.

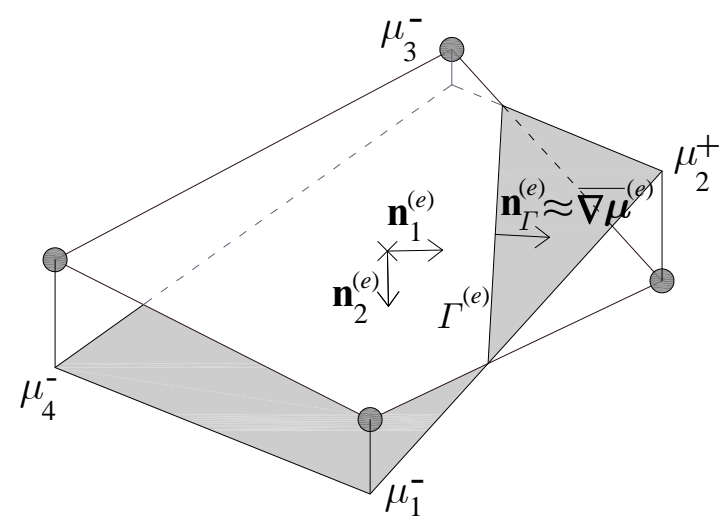

Figure A. 1 Determination of the crack path $\Gamma^{(e)}$, at the element level.

For an easier implementation, the explicit determination of the crack-path position and corresponding normal, $\mathbf{n}_{T}^{(e)}$, can be circumvented, by using, as an alternative to $\mathbf{n}_{T}^{(e)}$, the crack-path field gradient, $\overline{\nabla \boldsymbol{\mu}^{(e)}}$ evaluated at the finite element centroid (assuming $\left.\overline{\nabla \boldsymbol{\mu}^{(e)}} \approx \mathbf{n}_{\Gamma}^{(e)}\right)$. The gradient, $\overline{\nabla \boldsymbol{\mu}^{(e)}}$, can be easily determined for the time step $n+1$, as:

$$
\overline{\nabla \boldsymbol{\mu}_{n+1}^{(e)}}=[\overline{\nabla \mathbf{N}}]^{(e)} \cdot\{\boldsymbol{\mu}\}_{n+1}^{(e)} \text {. }
$$


where, $\{\boldsymbol{\mu}\}_{n+1}^{(e)}$ stands for element vector of degrees of freedom $\{\boldsymbol{\mu}\}_{n+1}^{(e)}=\left\{\mu_{1}, \mu_{2}, \mu_{3}, \mu_{4}\right\}_{n+1}^{T}$ and $[\nabla \mathbf{N}]^{(e)}$ stands for the elemental matrix of shape functions gradients.

BOX A-2 Normal selection

DATA : $\{\boldsymbol{\mu}\}_{n+1}, \mathbf{n}_{1}^{(\mathrm{e})}, \mathbf{n}_{2}^{(\mathrm{e})}, \mathbf{n}_{\Gamma}^{(e)}$

OUTPUT $: \mathbf{n}^{(\mathrm{e})}$

OPTION I (crack path criterion) $: \mathbf{n}^{(\mathrm{e})} \equiv \mathbf{n}_{\Gamma}^{(e)}$

OPTION II (bifurcation analysis criterion) :

1)Compute $\overline{\nabla \boldsymbol{\mu}_{n+10}^{(e)}}=\nabla \boldsymbol{\mu}_{n+1}^{(e)}\left(\mathbf{x}_{\mathcal{S}}\right)$

$$
\nabla \boldsymbol{\mu}_{n+1}^{(e)}\left(\mathbf{x}_{\mathcal{S}}\right)=\left[\nabla \mathbf{N}\left(\mathbf{x}_{\mathcal{S}}\right)\right]^{(e)} \cdot\{\boldsymbol{\mu}\}_{n+1}^{(e)}
$$

2) Compute dot products (absolute value)

$$
\operatorname{dotprod}_{1}=\left|\nabla \boldsymbol{\mu}_{n+1}^{(e)}\left(\mathbf{x}_{\mathcal{S}}\right) \cdot \mathbf{n}_{1}^{(\mathrm{e})}\right| ; \operatorname{dotprod}_{2}=\left|\nabla \boldsymbol{\mu}_{n+1}^{(e)}\left(\mathbf{x}_{\mathcal{S}}\right) \cdot \mathbf{n}_{2}^{(\mathrm{e})}\right|
$$

3) Select normal $\mathbf{n}^{(\mathrm{e})}$

$$
\mathbf{n}^{(\mathrm{e})}= \begin{cases}\mathbf{n}_{1}^{(\mathrm{e})} & \text { if } \text { dotprod }_{1} \geq \text { dotprod }_{2} \\ \mathbf{n}_{2}^{(\mathrm{e})} & \text { if } \text { dotprod }_{1}<\text { dotprod }_{2}\end{cases}
$$

The corresponding algorithms are displayed in BOX A-2. Results provided by both options are, in general, very similar for damage models, whereas for J2 and Rankine plasticity models OPTION II is preferable. In the examples presented in this work, the second option has been used.

\section{A.4 Crack-path-field technique: implementation aspects}

In this section some details about the implementation of the crack-path-field problem summarized in BOX 2.2-1 are given.

Introducing Voigt's notation, the rate version of equation (a) in BOX 2.2-1 reads:

$$
\begin{aligned}
& \mathcal{A} \underbrace{\left(\int_{\mathcal{B}^{(e)}} \mathbf{N}^{(e)^{T}} \cdot \mathbf{N}^{(e)} d \mathcal{B}\right)}_{M^{(e)}} \cdot\{\boldsymbol{\psi}\}=\mathcal{A}_{e}\left(\int_{\mathcal{B}^{(e)}} \mathbf{N}^{(e)^{T}} \cdot \dot{\alpha}_{t}^{(e)} d \mathcal{B}\right) \Leftrightarrow \\
& \Leftrightarrow M \cdot\{\dot{\boldsymbol{\psi}}\}_{t}=\underbrace{\mathcal{A}}_{e} \underbrace{\left(\int_{\mathcal{B}^{(e)}} \mathbf{N}^{(e)^{T}} \cdot \dot{\alpha}_{t}^{(e)} d \mathcal{B}\right)}_{\{\dot{\boldsymbol{h}}\}_{t}^{(e)}}
\end{aligned}
$$

where $\boldsymbol{M}$ stand for the global mass matrix $\boldsymbol{M}=\underset{e}{\mathcal{A}}\left(\boldsymbol{M}^{(e)}\right), \mathbf{N}^{(e)}$ stands for the element vector composed by the $N_{i}^{(e)}$ shape functions associated to node $i$ ( $\left.\mathbf{N}^{(e)}=\left\{\begin{array}{llll}N_{1}^{(e)} & N_{2}^{(e)} & N_{3}^{(e)} & N_{4}^{(e)}\end{array}\right\}\right), M^{(e)}$ stands for the element mass matrix, $\dot{\alpha}_{t}^{(e)}$ stands for the value of $\alpha$ associated to the element $(e)$ and $\{\dot{\psi}\}_{t}$ stands for the global vector composed by the $\psi_{i}$ degrees of freedom $\{\dot{\psi}\}_{t}=\left\{\dot{\psi}_{1}, \dot{\psi}_{2}, \ldots, \dot{\psi}_{\text {nnodes }}\right\}_{t}^{T}$ being nnodes the total number of nodes of the finite element mesh.

Substituting $\boldsymbol{M}$ by a lumped mass matrix version $\boldsymbol{M}_{L}$, equation A.(25) can be easily solved for $\{\dot{\boldsymbol{\psi}}\}_{t}$ as:

$$
\begin{aligned}
& \{\dot{\boldsymbol{\psi}}\}_{t}=\left(\boldsymbol{M}_{L}\right)^{-1} \cdot\{\dot{\boldsymbol{h}}\}_{t} \\
& \{\dot{\boldsymbol{h}}\}_{t}=\underset{e}{\mathcal{A}}\left(\{\dot{\boldsymbol{h}}\}_{t}^{(e)}\right), \quad\{\dot{\boldsymbol{h}}\}_{t}^{(e)}=\left\{\int_{\mathcal{B}^{(e)}} \mathbf{N}^{(e)^{T}} \dot{\alpha}_{t}^{(e)} d \mathcal{B}\right\}
\end{aligned}
$$


Alternatively to equation A.(26), an improved version, proposed by Weyler ${ }^{28}$, consisting of the introduction of a correction in the extrapolation of the Gauss point variable $\dot{\alpha}_{t}^{(e)}$ to the nodes, can be used. Then, as for equation A.(26), the extrapolated corrected nodal values are weighted by the corresponding nodal masses (lumped global mass matrix):

$$
\{\dot{\boldsymbol{\psi}}\}_{t}=\mathbf{M}_{L}^{-1} \cdot{\underset{e}{e}}_{\text {correction }}^{\mathcal{A}} \underbrace{(\boldsymbol{h}}_{\boldsymbol{M}^{(e)^{-1}} \cdot \boldsymbol{M}_{L}^{(e)}}\}_{t}^{(e)})
$$

Using the same procedure for equation $(b)$ in BOX 2.2-1

$$
\begin{aligned}
& \{\dot{\boldsymbol{\mu}}\}_{t}=\boldsymbol{M}_{L}^{-1} \cdot\{\dot{\boldsymbol{q}}\}_{t} \\
& \{\dot{\boldsymbol{q}}\}_{t}=\mathcal{A}_{e}^{\mathcal{A}}\{\dot{\boldsymbol{q}}\}_{t}^{(e)}, \quad\{\dot{\boldsymbol{q}}\}_{t}^{(e)}=\left\{\int_{\mathcal{B}^{(e)}} \mathbf{N}^{(e)^{T}} \frac{d \dot{\psi}_{t}^{(e)}}{d n} d \mathcal{B}\right\}
\end{aligned}
$$

being $\{\dot{\boldsymbol{\mu}}\}_{t}$ the global crack-path-field vector of degrees of freedom $\{\dot{\boldsymbol{\mu}}\}_{t}=\left\{\dot{\mu}_{1}, \dot{\mu}_{2}, \ldots, \dot{\mu}_{\text {nnode }}\right\}_{t}^{T}$. The improved version, reads:

$$
\{\dot{\boldsymbol{\mu}}\}_{t}=\boldsymbol{M}_{L}^{-1} \cdot \underset{e}{\mathcal{A}}\left(\boldsymbol{M}^{(e)^{-1}} \cdot \boldsymbol{M}_{L}^{(e)} \cdot\{\dot{\boldsymbol{q}}\}_{t}^{(e)}\right),
$$

The term, $\left(\frac{d \dot{\psi}_{t}}{d n}\right)^{(e)}$ in equation A. (28) can be computed on an element wise basis, once equation A.(27) is solved for $\{\dot{\boldsymbol{\psi}}\}_{t}$, as:

$$
\left(\frac{d \dot{\psi}_{t}}{d n}\right)^{(e)}=\left([\nabla \mathbf{N}]^{(e)} \cdot\{\dot{\boldsymbol{\psi}}\}_{t}^{(e)}\right) \cdot \mathbf{n}^{(e)}
$$

where $\{\dot{\boldsymbol{\psi}}\}_{t}^{(e)}$ is the element vector of degrees of freedom $\{\dot{\boldsymbol{\psi}}\}_{t}^{(e)}=\left\{\dot{\psi}_{1}, \dot{\psi}_{2}, \dot{\psi}_{3}, \dot{\psi}_{4}\right\}_{t}^{T}$ and $[\nabla \mathbf{N}]^{(e)}$ stands for the elemental matrix of shape functions gradients,

$$
[\nabla \mathbf{N}]^{(e)}=\left[\begin{array}{cccc}
\frac{\partial N_{1}}{\partial x} & \frac{\partial N_{2}}{\partial x} & \frac{\partial N_{3}}{\partial x} & \frac{\partial N_{4}}{\partial x} \\
\frac{\partial N_{1}}{\partial y} & \frac{\partial N_{2}}{\partial y} & \frac{\partial N_{3}}{\partial y} & \frac{\partial N_{4}}{\partial y}
\end{array}\right]
$$

Due to the evolutionary strain injection procedure, the term $\{\dot{\boldsymbol{h}}\}_{t}^{(e)}$ in equation A. (26) has to be computed trough specific quadratures depending on the injection domain to which element $e$ belongs, similarly to what has been introduced in A.(2), i.e:

where

$$
\begin{aligned}
& \int_{\mathcal{B}^{(e)}} \mathbf{N}^{(e)^{T}} \dot{\bar{\alpha}}_{t}^{(e)} d \mathcal{B} \approx \mathcal{I}_{\mathcal{B} \backslash \backslash \text { dis }}^{\text {Gass }}\left[\mathbf{N}^{(e)^{T}} \dot{\bar{\alpha}}_{t}^{(e)}\right] \quad \mathcal{B}^{(e)} \subset \mathcal{B} \backslash \mathcal{B}_{\text {inj }}(t) \\
& \int_{\mathcal{B}^{(e)}} \mathbf{N}^{(e)^{T}} \dot{\alpha}_{t}^{(e)} d \mathcal{B} \approx \mathcal{I}_{\text {Bloc }(t)}^{\text {CSM Injection }}\left[\mathbf{N}^{(e)^{T}} \dot{\alpha}_{t}^{(e)}\right] \quad \mathcal{B}^{(e)} \subset \mathcal{B}_{\text {loc }}(t) \\
& \int_{\mathcal{B}^{(e)}} \mathbf{N}^{(e)^{T}} \dot{\alpha}_{t}^{(e)} d \mathcal{B} \approx \mathcal{I}_{B_{\text {dis }}(t)}^{\text {DDMInjection }}\left[\mathbf{N}^{(e)^{T}} \dot{\alpha}_{t}^{(e)}\right]=\quad \mathcal{B}^{(e)} \subset \mathcal{B}_{\text {dis }}(t) \\
& \dot{\dot{\alpha}}_{t}^{(e)}=\tau_{t}^{(e)} \dot{\alpha}_{t}^{(e)}\left(\mathbf{x}_{i}\right)+\left(1-\tau_{t}^{(e)}\right) \dot{\bar{\alpha}}_{t}^{(e)} \\
& \dot{\bar{\alpha}}_{t}^{(e)} \approx \dot{\alpha}_{t}^{(e)}\left(\mathbf{x}_{\mathcal{S}}\right) \quad \mathcal{B}^{(e)} \subset \mathcal{B} \backslash \mathcal{B}_{\text {dis }}(t) \\
& \tau_{t}^{(e)}=\tau_{\text {stab }} \rightarrow\left\{\begin{array}{l}
\tau_{\text {stab }}=0.1 \rightarrow \text { isochoric case } \\
\tau_{\text {stab }}=1.0 \rightarrow \text { general case }
\end{array}\right.
\end{aligned}
$$

${ }^{28}$ R. Weyler, personal communication, 2012. 


$$
\begin{aligned}
& \mathcal{I}_{\mathcal{B} \backslash \mathbb{B}_{\text {inj }}(t)}^{\text {Gauss }}\left[\mathbf{N}^{(e)^{T}} \dot{\bar{\alpha}}_{t}^{(e)}\right]=\sum_{i=1}^{i=4} \mathbf{N}^{(e)^{T}}\left(\mathbf{x}_{i}\right) \dot{\bar{\alpha}}_{t}^{(e)}\left(\mathbf{x}_{i}\right) w_{i}\left(\mathbf{x}_{i}\right) \\
& \mathcal{I}_{\mathcal{B}_{\text {loc }}(t)}^{\text {CSM Injection }}\left[\mathbf{N}^{(e)^{T}} \dot{\alpha}_{t}^{(e)}\right]=\mathbf{N}^{(e)^{T}}\left(\mathbf{x}_{C}\right) \dot{\bar{\alpha}}_{t}^{(e)} \operatorname{meas}\left(\mathcal{B}^{(e)}\right) \\
& \mathcal{I}_{\mathcal{B}_{\text {dis }}(t)}^{\text {DDMInjection }}\left[\mathbf{N}^{(e)^{T}} \dot{\alpha}_{t}^{(e)}\right]=\mathbf{N}^{(e)^{T}}\left(\mathbf{x}_{\mathcal{S}}\right) \dot{\alpha}_{t}^{(e)}\left(\mathbf{x}_{\mathcal{S}}\right) W\left(\mathbf{x}_{\mathcal{S}}\right)
\end{aligned}
$$

where $w\left(\mathbf{x}_{i}\right), i=(1,2,3,4)$ are the weights corresponding to the four-point Gauss integration, meas $\left(\mathcal{B}^{(e)}\right)$ the weight corresponding to the barycenter-injection point and $W\left(\mathbf{x}_{\mathcal{S}}\right)$ are the weight of the corresponding injection point in Table 4.2.1-1.

For purposes of numerical integration in time, it is convenient to rephrase the functionals in A.(33) in terms of some equivalent values of the strain like internal variable, $\dot{\alpha}^{(e)}$. The equivalent values, $\dot{\tilde{\alpha}}^{(e)}$, are computed at the standard Gauss points according with a procedure similar to that presented in section A.1.

\section{A.4.1 Rephrased numerical quadrature at the CSM injection domain $\mathcal{B}_{\text {loc }}(t)$}

Following a similar procedure to that used in section A.1.1., the term in equation A.(33)(b) can be rephrased in terms of some equivalent values, $\dot{\tilde{\alpha}}_{t}^{(e)}\left(\mathbf{x}_{i}\right)$, of the internal strain variable $\dot{\alpha}_{t}^{(e)}$ :

$$
\dot{\tilde{\alpha}}_{t}^{(e)}\left(\mathbf{x}_{i}\right)=\dot{\bar{\alpha}}_{t}^{(e)} \approx \dot{\alpha}_{t}^{(e)}\left(\mathbf{x}_{\mathcal{S}}\right) \quad, i=(1,2,3,4), \forall \mathcal{B}^{(e)} \subset \mathcal{B}_{\text {loc }}(t)
$$

fulfilling the following property:

$$
\mathcal{I}_{\mathcal{B}_{\text {loc }}(t)}^{\text {CSM injection }}\left[\mathbf{N}^{(e)^{T}} \dot{\alpha}_{t}^{(e)}(\mathbf{x})\right]=\mathcal{I}_{\mathcal{B}_{\text {loc }}(t)}^{\text {Gauss }}\left[\mathbf{N}^{(e)^{T}}(\mathbf{x}) \dot{\tilde{\alpha}}_{t}^{(e)}(\mathbf{x})\right]
$$

\section{A.4.2 Rephrased numerical quadrature at the DDM injection domain $\mathcal{B}_{\text {dis }}(t)$}

The term in equation A.(33)-(c), is now rephrased trough the following change of variable $\dot{\hat{\boldsymbol{a}}}_{t}^{(e)}$ :

$$
\dot{\hat{\alpha}}_{t}^{(e)}=\frac{k^{(e)} \dot{\alpha}_{t}^{(e)}}{\ell^{(e)}}=\xi \dot{\alpha}_{t}^{(e)}
$$

such that:

$$
\begin{aligned}
& \mathcal{I}_{B_{\text {dis }}(t)}^{\text {DDM Injection }}\left[\mathbf{N}^{(e)^{T}} \dot{\alpha}_{t}^{(e)}\right]=\underbrace{\mathbf{N}^{(e)^{T}}\left(\mathbf{x}_{\mathcal{S}}\right)}_{\left.\overline{\left(\mathbf{N}^{(e)}\right.}\right)^{T}} \dot{\alpha}_{t}^{(e)}\left(\mathbf{x}_{\mathcal{S}}\right) W\left(\mathbf{x}_{\mathcal{S}}\right)=\overline{\left(\mathbf{N}^{(e)}\right.})^{T} \underbrace{\dot{\alpha}_{t}^{(e)} k^{(e)}}_{\dot{\hat{\alpha}}_{t}^{(e)} \ell^{(e)}} \operatorname{meas}\left(\mathcal{S}^{(e)}\right)= \\
& \left.=\overline{\left(\mathbf{N}^{(e)}\right.}\right)^{T} \dot{\hat{\alpha}}^{(e)} \underbrace{\ell^{(e)} \operatorname{meas}\left(\mathcal{S}^{(e)}\right)}_{\operatorname{meas}\left(\mathcal{B}^{(e)}\right)}=\underbrace{\left.\overline{\left(\mathbf{N}^{(e)}\right.}\right)^{T} \operatorname{meas}\left(\mathcal{B}^{(e)}\right)}_{\sum_{i=1}^{i=4} \mathbf{N}^{(e)^{T}}\left(\mathbf{x}_{i}\right) \cdot w_{i}\left(\mathbf{x}_{i}\right)} \cdot \dot{\hat{\alpha}}^{(e)}= \\
& =\sum_{i=1}^{i=4} \mathbf{N}^{(e)^{T}}\left(\mathbf{x}_{i}\right) w_{i}\left(\mathbf{x}_{i}\right) \cdot \underbrace{\dot{\hat{\alpha}}^{(e)}}_{\begin{array}{c}
\text { constant } \\
\text { at all } \mathbf{x}_{i}
\end{array}}=\mathcal{T}_{\mathcal{B}_{\text {dis }}(t)}^{\text {Gauss }}\left[\mathbf{N}^{(e)^{T}} \dot{\hat{\alpha}}^{(e)}(\mathbf{x})\right]
\end{aligned}
$$

where $\overline{\mathbf{N}^{(e)^{T}}}$ stands for the mean value of $\mathbf{N}^{(e)^{T}}$ and $\ell^{(e)}$ is the finite element characteristic length (according with (Oliver 1989)). In equation A.(37) the continuous character of the shape functions and the fact that the integration point $\mathbf{x}_{\mathcal{S}}$ is placed at the finite element centroid is take into consideration.

Results in equation A.(37) involve the definition of some new equivalent values $\dot{\tilde{\alpha}}_{t}^{(e)}$ computed in $\mathcal{B}_{\text {dis }}(t)$, at the standard Gauss points, $\dot{\tilde{\alpha}}_{t}^{(e)}\left(\mathbf{x}_{i}\right)$, through:

$$
\dot{\tilde{\alpha}}_{t}^{(e)}\left(\mathbf{x}_{i}\right) \equiv \dot{\hat{\alpha}}_{t}^{(e)}=\xi \dot{\alpha}_{t}^{(e)} \quad i=(1,2,3,4), \quad \forall \mathcal{B}^{(e)} \subset \mathcal{B}_{\text {dis }}(t)
$$


fulfilling the following property:

$$
\mathcal{I}_{\mathcal{B}_{\text {dis }}(t)}^{\text {DD injection }}\left[\mathbf{N}^{(e)^{T}} \dot{\alpha}_{t}^{(e)}(\mathbf{x})\right]=\mathcal{I}_{\mathcal{B}_{\text {dis }}^{\text {Gauss }}}^{\text {at }}\left[\mathbf{N}^{(e)^{T}}(\mathbf{x}) \dot{\tilde{\alpha}}_{t}^{(e)}(\mathbf{x})\right]
$$

In equation A.(36), $\dot{\hat{\alpha}}_{t}^{(e)}$ can be interpreted as a distributed version, of the strong localized variable $\dot{\alpha}_{t}^{(e)}$, over the element bandwidth $\ell^{(e)}$ (see Figure A.1).

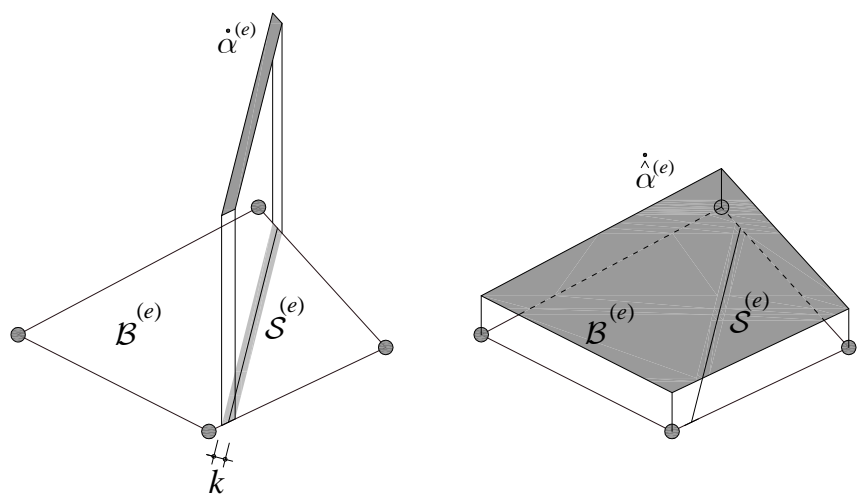

Figure A. 2 Interpretation of variable $\dot{\hat{\boldsymbol{a}}}_{t}^{(e)}$.

\section{A.4.3 Rephrased numerical quadrature at the remaining domain $\mathcal{B} \backslash \mathcal{B}_{\text {inj }}(t)$}

The term in equation A.(33)-(a) is already a four-point Gauss quadrature. Therefore, the following equivalence is straightforward:

$$
\dot{\tilde{\alpha}}_{t}^{(e)}\left(\mathbf{x}_{i}\right)=\dot{\tilde{\alpha}}_{t}^{(e)}\left(\mathbf{x}_{i}\right) \quad, i=(1,2,3,4), \forall \mathcal{B}^{(e)} \subset \mathcal{B} \backslash \mathcal{B}_{\text {inj }}(t)
$$

fulfilling the following property:

$$
\mathcal{I}_{\mathcal{B} \backslash \backslash_{\text {inj }}(t)}^{\text {Gaus }}\left[\mathbf{N}^{(e)^{T}}(\mathbf{x}) \dot{\tilde{\alpha}}_{t}^{(e)}(\mathbf{x})\right]=\mathcal{I}_{\mathcal{B} \backslash \backslash_{\text {inj }}(t)}^{\text {Gaus }}\left[\mathbf{N}^{(e)^{T}}(\mathbf{x}) \dot{\tilde{\alpha}}_{t}^{(e)}(\mathbf{x})\right]
$$

A.4.4 Unified numerical quadrature at the total domain $\mathcal{B}=\left(\mathcal{B} \backslash \mathcal{B}_{\text {inj }}(t)\right) \cup \mathcal{B}_{\text {loc }}(t) \cup \mathcal{B}_{\text {dis }}(t)$

Using the results of equations A.(35) A.(39) and A.(41), the term $\{\dot{\boldsymbol{h}}\}_{t}^{(e)}$, in equation A. (26) can be rewritten in terms of the equivalent/effective values in equations A.(34),A.(38) and A.(40)) yielding,

$$
\begin{aligned}
& \{\dot{\boldsymbol{h}}\}_{t}=\mathcal{A}_{e}\left(\{\dot{\boldsymbol{h}}\}_{t}^{(e)}\right), \\
& \{\dot{\boldsymbol{h}}\}_{t}^{(e)} \approx\left\{\mathcal{I}_{\mathcal{B}^{(e)}}^{\text {Gauss }}\left[\mathbf{N}^{(e)^{T}}(\mathbf{x}) \dot{\tilde{\alpha}}_{t}^{(e)}(\mathbf{x})\right]\right\} \\
& \dot{\tilde{\alpha}}_{t}^{(e)}\left(\mathbf{x}_{i}\right)= \begin{cases}\tau_{t}^{(e)} \dot{\alpha}_{t}^{(e)}\left(\mathbf{x}_{i}\right)+\left(1-\tau_{t}^{(e)}\right) \dot{\alpha}_{t}^{(e)}\left(\mathbf{x}_{\mathcal{S}}\right) & \mathcal{B}^{(e)} \subset \mathcal{B} \backslash \mathcal{B}_{i n j} \\
\dot{\alpha}_{t}^{(e)}\left(\mathbf{x}_{\mathcal{S}}\right) & \mathcal{B}^{(e)} \subset \mathcal{B}_{l o c} \\
\xi \dot{\alpha}_{t}^{(e)}\left(\mathbf{x}_{\mathcal{S}}\right) & \mathcal{B}^{(e)} \subset \mathcal{B}_{\text {dis }}\end{cases} \\
& \tau_{t}^{(e)}=\tau_{\text {stab }} \rightarrow \begin{cases}\tau_{\text {stab }}=0.1 \rightarrow \text { isochoric case } \\
\tau_{\text {stab }}=1.0 \rightarrow \text { general case }\end{cases}
\end{aligned}
$$

Then, equation A.(42)-(a) can be trivially integrated in time, considering the initial conditions $\left.\tilde{\alpha}_{t}^{(e)}(\mathbf{x})\right|_{t=0}=\mathbf{0}$, yielding,

$$
\{\boldsymbol{h}\}_{n+1}^{(e)}=\left\{\int_{\mathcal{B}^{(e)}} \mathbf{N}^{(e)^{T}} \tilde{\alpha}_{n+1}^{(e)} d \mathcal{B}\right\} \approx\left\{\mathcal{I}_{\mathcal{B}_{\text {loc }}(t)}^{\text {Gauss }}\left[\mathbf{N}^{(e)^{T}}(\mathbf{x}) \tilde{\alpha}_{n+1}^{(e)}(\mathbf{x})\right]\right\}
$$


where the effective values, $\tilde{\alpha}_{n+1}^{(e)}(\mathbf{x})$, are incrementally computed through time integration of equations A.(42)-(b). i.e.,

$$
\begin{aligned}
& \tilde{\alpha}_{n+1}^{(e)}\left(\mathbf{x}_{i}\right)=\tilde{\alpha}_{n}^{(e)}\left(\mathbf{x}_{i}\right)+\Delta \tilde{\alpha}_{n+1}^{(e)}\left(\mathbf{x}_{i}\right) \quad ; \mathbf{x}_{i}(i=1, \ldots, 4) \\
& \Delta \tilde{\alpha}_{n+1}^{(e)}\left(\mathbf{x}_{i}\right)= \begin{cases}\tau_{n+1}^{(e)} \Delta \alpha_{n+1}^{(e)}\left(\mathbf{x}_{i}\right)+\left(1-\tau_{n+1}^{(e)}\right) \Delta \alpha_{n+1}^{(e)}\left(\mathbf{x}_{\mathcal{S}}\right) & \mathcal{B}^{(e)} \subset \mathcal{B} \backslash \mathcal{B}_{i n j} \\
\Delta \alpha_{n+1}^{(e)}\left(\mathbf{x}_{\mathcal{S}}\right) & \mathcal{B}^{(e)} \subset \mathcal{B}_{l o c} \\
\xi \Delta \alpha_{n+1}^{(e)}\left(\mathbf{x}_{\mathcal{S}}\right) & \mathcal{B}^{(e)} \subset \mathcal{B}_{\text {dis }}\end{cases} \\
& \tau_{n+1}^{(e)}=\tau_{\text {stab }} \rightarrow\left\{\begin{array}{l}
\tau_{\text {stab }}=0.1 \rightarrow \text { isochoric case } \\
\tau_{\text {stab }}=1.0 \rightarrow \text { general case }
\end{array}\right.
\end{aligned}
$$

Finally, the suitable for implementation matrix equations of the crack-path-field problem are summarized in BOX A-3.

BOX A-3 Crack-path-field - algorithm.

$$
\begin{aligned}
& \text { DATA: } \tilde{\alpha}_{n}^{(e)}\left(\mathbf{x}_{i}\right), \alpha_{n}^{(e)}\left(\mathbf{x}_{i}\right), \hat{\alpha}_{n}^{(e)}\left(\mathbf{x}_{\mathcal{S}}\right), \alpha_{n+1}^{(e)}\left(\mathbf{x}_{i}\right), \alpha_{n+1}^{(e)}\left(\mathbf{x}_{\mathcal{S}}\right) \quad(i=1, \ldots, 4) \\
& \text { OUTPUT : } \tilde{\alpha}_{n+1}^{(e)}\left(\mathbf{x}_{i}\right),\{\boldsymbol{\mu}\}_{n+1} \\
& \text { 1)Compute }\{\boldsymbol{\psi}\}_{n+1} \\
& \{\boldsymbol{\psi}\}_{n+1}=\boldsymbol{M}_{L}^{-1} \cdot \underbrace{\mathcal{A}}_{e}(\underbrace{\boldsymbol{M}^{(e)-1} \cdot \boldsymbol{M}_{L}^{(e)}}_{\text {correction }} \cdot\{\boldsymbol{h}\}_{n+1}^{(e)}) \\
& \{\boldsymbol{h}\}_{n+1}^{(e)}=\left\{\int_{\mathcal{B}^{(e)}} \mathbf{N}^{(e)^{T}}(\mathbf{x}) \cdot \tilde{\alpha}_{n+1}^{(e)}(\mathbf{x}) d \mathcal{B}\right\}
\end{aligned}
$$

where:

$$
\tilde{\alpha}_{n+1}^{(e)}\left(\mathbf{x}_{i}\right)=\tilde{\alpha}_{n}^{(e)}\left(\mathbf{x}_{i}\right)+\Delta \tilde{\alpha}_{n+1}^{(e)}\left(\mathbf{x}_{i}\right) \quad ; \mathbf{x}_{i}(i=1,2,3,4)
$$

being:

$\Delta \tilde{\alpha}_{n+1}^{(e)}\left(\mathbf{x}_{i}\right)= \begin{cases}\tau_{n+1}^{(e)} \Delta \alpha_{n+1}^{(e)}\left(\mathbf{x}_{i}\right)+\left(1-\tau_{n+1}^{(e)}\right) \Delta \alpha_{n+1}^{(e)}\left(\mathbf{x}_{\mathcal{S}}\right) & \mathcal{B}^{(e)} \subset \mathcal{B} \backslash \mathcal{B}_{i n j} \\ \Delta \alpha_{n+1}^{(e)}\left(\mathbf{x}_{\mathcal{S}}\right) & \mathcal{B}^{(e)} \subset \mathcal{B}_{l o c} \\ \xi \Delta \alpha_{n+1}^{(e)}\left(\mathbf{x}_{\mathcal{S}}\right) & \mathcal{B}^{(e)} \subset \mathcal{B}_{\text {dis }}\end{cases}$

$\tau_{n+1}^{(e)}=\tau_{\text {stab }} \rightarrow\left\{\begin{array}{l}\tau_{\text {stab }}=0.1 \rightarrow \text { isochoric case } \\ \tau_{\text {stab }}=1.0 \rightarrow \text { general case }\end{array}\right.$

2) Compute $\{\boldsymbol{\mu}\}_{n+1}$

$$
\begin{gathered}
\{\boldsymbol{\mu}\}_{n+1}=\boldsymbol{M}_{L}^{-1} \cdot \underset{e}{\mathcal{A}}\left(\boldsymbol{M}^{(e)^{-1}} \cdot \boldsymbol{M}_{L}^{(e)} \cdot \int_{\mathcal{B}^{(e)}} \mathbf{N}^{(e)^{T}}(\mathbf{x}) \cdot\left(\frac{d \psi}{d n}(\mathbf{x})\right)_{n+1}^{(e)} d \mathcal{B}\right): \\
\left(\left.\frac{d \psi}{d n}(\mathbf{x})\right|_{n+1} ^{(e)}=\left([\nabla \mathbf{N}(\mathbf{x})]^{(e)} \cdot\{\boldsymbol{\psi}\}_{n+1}^{(e)}\right) \cdot \mathbf{n}^{(e)}\right.
\end{gathered}
$$

\section{A.5 Algorithmic management of the injection domains: injection-switching strategy.}

This appendix systematizes, for implementation purposes, the algorithmic treatment of given element, $\mathcal{B}^{(e)}$, when it moves from one to another the injection domains $\mathcal{B} \backslash \mathcal{B}_{\text {inj }}, \mathcal{B}_{\text {loc }}$ and $\mathcal{B}_{\text {dis }}$. It is based on the following ingredients:

1) The elemental injection status indicator $I S_{n+1}^{(e)} \in\{0,1,2\}$ defines the status of element $\mathcal{B}^{(e)}$, during the time interval $\left[t_{n}, t_{n+1}\right]$, in terms of its belonging to the injection domains $\mathcal{B} \backslash \mathcal{B}_{\text {inj }}, \mathcal{B}_{\text {loc }}$ and $\mathcal{B}_{\text {dis }}$ (see Table A-1). 
Table A-1. Injection status indicator

\begin{tabular}{|l|c|c|}
\hline \multicolumn{1}{|c|}{ INJECTION STATUS } & $\begin{array}{c}\text { INJECTION DOMAIN } \\
\text { BELONGING }\end{array}$ & $\begin{array}{c}\text { INJECTION STATUS } \\
\text { INDICATOR }\end{array}$ \\
\hline No strain injection & $\mathcal{B}^{(e)} \subset \mathcal{B} \backslash \mathcal{B}_{\text {inj }}\left(t_{n+1}\right)$ & $(I S)_{n+1}^{(e)}=0$ \\
\hline $\begin{array}{l}\text { Localized Constant Strain } \\
\text { Mode (CSM) injection }\end{array}$ & $\mathcal{B}^{(e)} \subset \mathcal{B}_{\text {loc }}\left(t_{n+1}\right)$ & $(I S)_{n+1}^{(e)}=1$ \\
\hline $\begin{array}{l}\text { Discontinuous displacement } \\
\text { mode (DDM) injection }\end{array}$ & $\mathcal{B}^{(e)} \subset \mathcal{B}_{\text {dis }}\left(t_{n+1}\right)$ & $(I S)_{n+1}^{(e)}=2$ \\
\hline
\end{tabular}

2) A set of flags $(\bullet) \in\{0,1\}$ (evaluated at the end of the preceeding time step) are used to control the belonging of element $\mathcal{B}^{(e)}$ to the injection domains during the current time interval $\left[t_{n}, t_{n+1}\right]$ (see Table A-2)

Table A-2. Injection flags

\begin{tabular}{|c|c|c|}
\hline INDICATOR NAME & $\begin{array}{l}F L A G \\
L A B E L\end{array}$ & INDICATOR DEFINITION \\
\hline Bifurcation Flag & $B F$ & $(B F)_{n}^{(e)}=\left\{\begin{array}{lll}0 & \text { if } & t_{n}<t_{B}^{(e)}\left(\mathbf{x}_{S}\right) \\
1 & \text { if } & t_{n} \geq t_{B}^{(e)}\left(\mathbf{x}_{S}\right)\end{array}\right.$ \\
\hline Loading/unloading Flag & $L F$ & $(L F)_{n}^{(e)}=\left\{\begin{array}{lll}0 & \text { if } & \Delta r_{n}^{(e)}\left(\mathbf{x}_{S}\right)=0 \\
1 & \text { if } & \Delta r_{n}^{(e)}\left(\mathbf{x}_{S}\right)>0\end{array}\right.$ \\
\hline Softening degree Flag & $S F$ & $(S F)_{n}^{(e)}=\left\{\begin{array}{lll}0 & \text { if } & q_{n}^{(e)}\left(\mathbf{x}_{S}\right)>q_{i n j} \\
1 & \text { if } & q_{n}^{(e)}\left(\mathbf{x}_{S}\right) \leq q_{i n j}\end{array}\right.$ \\
\hline Crack path Flag & $C F$ & $(C F)_{n}^{(e)}=\left\{\begin{array}{l}1 \text { if element } e \text { is crossed by the crack } \\
\quad \text { path } \Gamma_{n}(\text { accross two sides }) \\
0 \text { otherwise }\end{array}\right.$ \\
\hline
\end{tabular}

3) The injection status of element (IS $)_{n+1}^{(e)}$ in Table A-1 is set in terms of specific products of the flags in Table A-2, according to Table A-3. Notice that check conditions in Table A-3 stem directly from the definitions of the injection domains $\mathcal{B} \backslash \mathcal{B}_{\text {inj }}, \mathcal{B}_{\text {loc }}$ and $\mathcal{B}_{\text {dis }}$ (see Section 4.2.4).

Table A-3 Injection status setting

\begin{tabular}{|c|c|c|}
\hline \multirow{2}{*}{$\begin{array}{c}\text { INJECTION } \\
\text { STATUS } \\
\text { time-step } n+1) \\
(I S)_{n+1}^{(e)}\end{array}$} & $(B F)_{n}^{(e)} \times(L F)_{n}^{(e)}$ & \multicolumn{2}{|c|}{$\begin{array}{c}\text { CHECK CONDITIONS } \\
(\text { time-step } n)\end{array}$} \\
\cline { 2 - 3 } & 0 & $(S F)_{n}^{(e)} \times(C F)_{n}^{(e)}$ \\
\hline 0 & 1 & 0 \\
\hline 1 & 1 & 1 \\
\hline 2 & & -1 \\
\hline
\end{tabular}




\section{Appendix B. Stress-locking issues in finite elements with embedded discontinuities}

Let us consider a strong discontinuity approach based on finite element quadrilaterals with constant embedded discontinuities. The strong discontinuity kinematics in equations (40) and (44) is described in terms of a two-field, $\dot{\hat{u}}, \llbracket \dot{u} \rrbracket$, approach where the generalized displacement filed, $\dot{\hat{u}}$, is bilinear and the displacement jump $\llbracket \dot{u} \rrbracket$ is element-wise constant. Under these conditions, integration of the resulting equations requires a standard four points-based Gauss quadrature for integration of the terms in equation (66)-(a) in $\mathcal{B}^{(e)} \backslash \mathcal{S}$ plus one additional sampling point, $\mathbf{x}_{\mathcal{S}}$, for the integration in $\mathcal{S}^{(e)}$ (see Figure B. 1.-(a)).

It is well know that, in this formulation, the appearance of stress locking is noticeable specially when trying to reproduce non-constant separation modes along the element discontinuity ${ }^{29}$. This issue was analysed in (Linder and Armero 2007) and (Manzoli and Shing 2006) where the authors propose elements with linear interpolation in the displacement jump to circumvent the problem.

In this work, instead, the displacement discontinuity mode (DDM) injection in the domain $\mathcal{B}_{\text {dis }}(t)$ is introduced via the three field $\dot{\hat{u}}, \dot{\bar{\varepsilon}}, \llbracket \dot{u} \rrbracket$ mixed formulation in section 4.2.2 and this fact translates into an element-wise-constant regular strain field.. The corresponding variational form in equations (66) requires only two sampling points (singular and regular) in Figure B. 1.-(b).

(a)

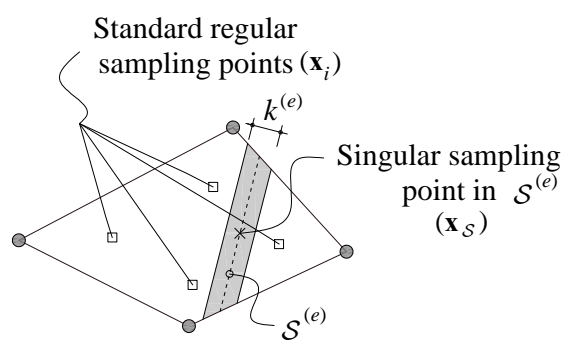

(b)

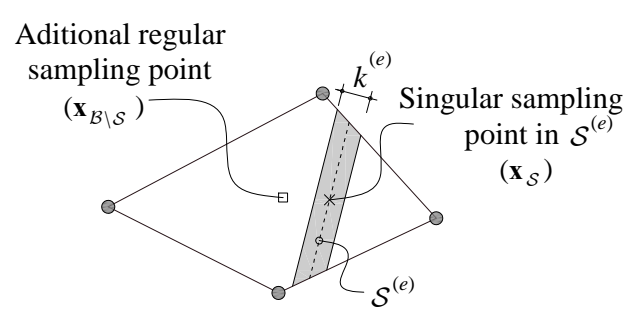

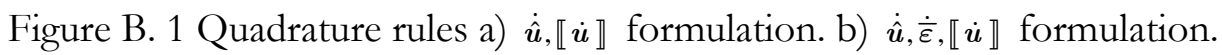

The interesting practical property of using the here proposed $\dot{\hat{u}}, \dot{\bar{\varepsilon}}, \llbracket \dot{u} \rrbracket$ constant ump/constant-strain formulation in quadrilateral elements, is that the element (unlike the $\dot{\hat{u}}, \llbracket \dot{u} \rrbracket$ case) is stress locking free. In next sections an explanation for this improved behavior is given.

\section{B.1 Motivation}

We assume that for an element to be stress locking free its kinematics should allow rigid body motions of one part of the element with respect to the other (Figure B. 2), when the discontinuity is fully open (full softening, stress released state, of the element).

\footnotetext{
${ }^{29}$ Though mesh refinement tend to strongly reduce this stress locking.
} 


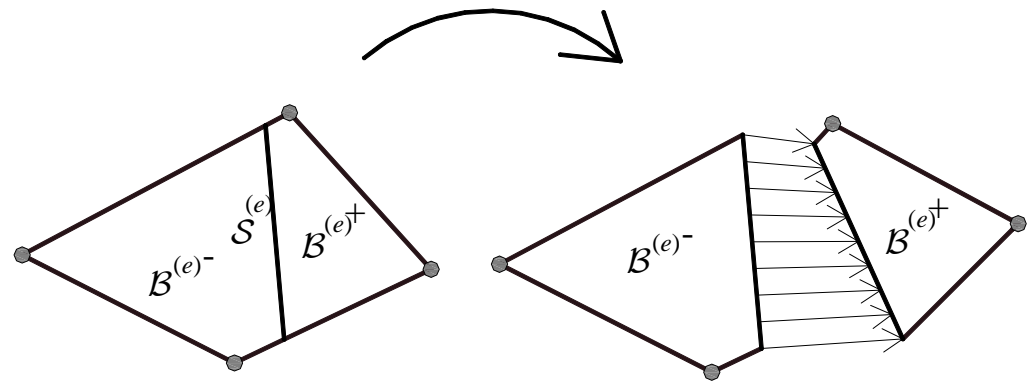

Figure B. 2 Quadrilateral element with a discontinuity inducing a rigid body motion in $\mathcal{B}^{(e)^{+}}$with respect to $\mathcal{B}^{(e)^{-}}$.

Recalling the strong discontinuity kinematics of equation (41)

$$
\dot{u}(\mathbf{x})=\dot{\bar{u}}(\mathbf{x})+\mathcal{H}_{\mathcal{S}} \llbracket \dot{u} \rrbracket(\mathbf{x}),
$$

where $\dot{\bar{u}}$ stands for the smooth part of the displacement field and $\mathcal{H}_{\mathcal{S}}$ stands for the Heaviside (step) function $\left(\mathcal{H}_{\mathcal{S}}(\mathbf{x})=0, \forall \mathbf{x} \in \mathcal{B}^{-}\right.$and $\left.\mathcal{H}_{\mathcal{S}}(\mathbf{x})=1, \forall \mathbf{x} \in \mathcal{B}^{+}\right)$.

For the stress-released case we assume $\dot{\bar{u}}=\mathbf{0}$, thus:

$$
\dot{u}(\mathbf{x})=\mathcal{H}_{\mathcal{S}} \llbracket \dot{u} \rrbracket(\mathbf{x})\left\{\begin{array}{l}
\left.\dot{u}(\mathbf{x})\right|_{\mathbf{x} \in \mathcal{B}^{-}}=0 \\
\left.\dot{u}(\mathbf{x})\right|_{\mathbf{x} \in \mathcal{B}^{+}}=\llbracket \dot{u} \rrbracket(\mathbf{x})
\end{array}\right.
$$

For the general three-dimensional case, the rigid body motions of $\mathcal{B}^{(e)^{+}}$in the stressreleased state requires the displacement $\llbracket \dot{\boldsymbol{u}} \rrbracket(\mathbf{x})$ in equation B.(47) being described as a rigid body motion in terms of 6 degrees of freedom: 3 displacements (traslation) and 3 rotations, i.e.,

$$
\llbracket \dot{\boldsymbol{u}} \rrbracket(\mathbf{x})=\boldsymbol{c}+\boldsymbol{\theta} \wedge \mathbf{x},
$$

where $\boldsymbol{c}=\left\{c_{1}, c_{2}, c_{3}\right\}^{T}$ and $\boldsymbol{\theta}=\left\{\theta_{1}, \theta_{2}, \theta_{3}\right\}^{T}$ are vectors of degree of freedoms associated with traslation and rotation, respectively. Notice that the displacement jump in equation B.(48) linearly depends on $\mathbf{x}$.

Equation B.(48) can be written in matrix form, for element $e$, as:

$$
\llbracket \dot{\boldsymbol{u}} \rrbracket^{(e)}(\mathbf{x})=\underbrace{\left[\begin{array}{ccc|ccc}
1 & 0 & 0 & 0 & x_{3} & -x_{2} \\
0 & 1 & 0 & -x_{3} & 0 & x_{1} \\
0 & 0 & 1 & x_{2} & -x_{1} & 0
\end{array}\right]}_{\boldsymbol{G}^{(e)}(\mathbf{x})} \underbrace{\left[\begin{array}{c}
c_{1} \\
c_{2} \\
c_{3} \\
\theta_{1} \\
\theta_{2} \\
\theta_{3}
\end{array}\right]}_{\boldsymbol{\delta}^{(e)}}=\boldsymbol{G}^{(e)}(\mathbf{x}) \cdot \boldsymbol{\delta}^{(e)}
$$

where $x_{i},(i=1,2,3)$ stand for the Cartesian coordinates measured with respect to an (arbitrary) origin of coordinates. In a two-dimensional framework, equation B.(49) simplifies to:

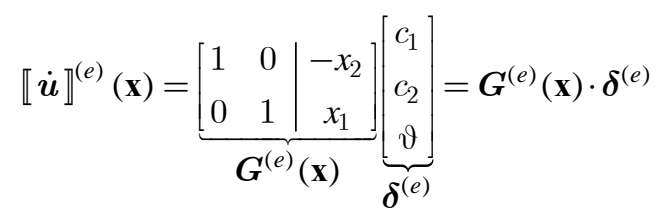

\section{B.2 Specification for 2D quadrilateral elements}

Since rigid body modes are allowed for the full stress-released state, any finite element formulation admitting linear jumps, like the one in equation B.(50) should be stress locking free disregard the type of finite element (triangles, quadrilaterals etc.). 
It is evident that the kinematics bilinear quadrilateral with a constant-jump embedded discontinuity, $(\dot{\hat{\boldsymbol{u}}}, \llbracket \dot{\boldsymbol{u}} \rrbracket)$, with a $2 \times 2$ quadrature rule in $\mathcal{B}^{(e)} \backslash \mathcal{S}$ and the additional sampling point in $\mathcal{S}^{(e)}\left(\mathbf{x}_{\mathcal{S}}\right)$, does not fulfill equation B.(50) at all sampling points for an arbitrary (constant) $\llbracket \dot{u} \rrbracket^{(e)}$ (see Figure B. 3) ${ }^{30}$. Therefore, rigid body modes cannot develop for the general deformation case and the element can potentially suffer from stress locking.

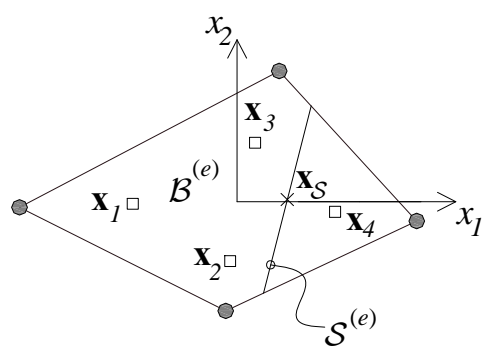

Figure B. 3 Quadrilateral element associated to a $\dot{\hat{u}}, \llbracket \dot{u} \rrbracket$ formulation.

Instead, for the DDM injection quadrilateral element proposed in this work, with only the two regular/singular sampling points, condition B.(50) for the proposed (constant) $\llbracket \dot{u} \rrbracket^{(e)}$, can be trivially fulfilled at the two sampling points for any arbitrary $\llbracket \dot{\boldsymbol{u}} \rrbracket^{(e)}$. The proof is rather simple: since the origin of coordinates is arbitrary, let us take this origin at $\mathbf{x}_{\mathcal{S}}=\mathbf{x}_{\mathcal{B} \backslash \mathcal{S}}(=\mathbf{0})$ (see Figure B.4).

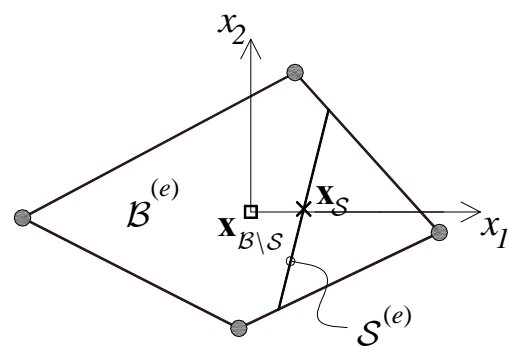

Figure B.4 Quadrilateral element associated to a $\dot{\hat{u}}, \dot{\hat{\varepsilon}}, \llbracket \dot{u} \rrbracket$ formulation.

The third column of the operator $G(\mathbf{x})$ (in equation B.(50)), when evaluated at the two sampling points, is null and

$$
\boldsymbol{G}(\mathbf{x})=\left[\begin{array}{ll|l}
1 & 0 & 0 \\
0 & 1 & 0
\end{array}\right] \Rightarrow \llbracket \dot{\boldsymbol{u}} \rrbracket^{(e)}=\left\{\begin{array}{l}
c_{1} \\
c_{2}
\end{array}\right\}
$$

The solution is $\left\{c_{1}, c_{2}\right\}^{T}=\llbracket \dot{\boldsymbol{u}} \rrbracket^{(e)}$, and the rotational degree of freedom, $\theta$, does not appear in the formulation. This is precisely the proposed kinematics and, therefore, the resulting finite element should be stress-locking free.

\section{B.3 Numerical example}

The following numerical example, already considered in (Linder and Armero 2007) and (Manzoli and Shing 2006) (see Figure B. 5), illustrates the behavior of quadrilaterals with, constant jump embedded discontinuities in front of stress locking.

The test consists of a square block with imposed displacement at the top, $\delta_{\text {top }}$, and bottom, $\delta_{\text {bot }}$, with $\delta_{\text {bot }}$ increasing twice as the top displacement in the loading pseudo-time $t(s)$.

${ }^{30}$.In fact, the only solution for equation B.(50) in this case is $\dot{\boldsymbol{\delta}}^{(e)}=\mathbf{0} \Rightarrow \llbracket \dot{\boldsymbol{u}} \rrbracket^{(e)}=\mathbf{0}$. 
a)

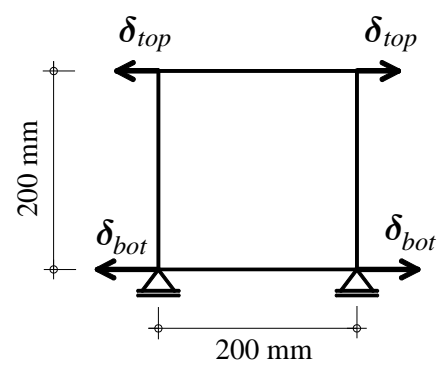

b)

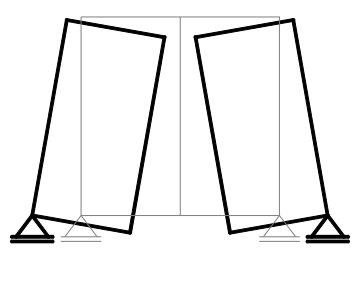

c)

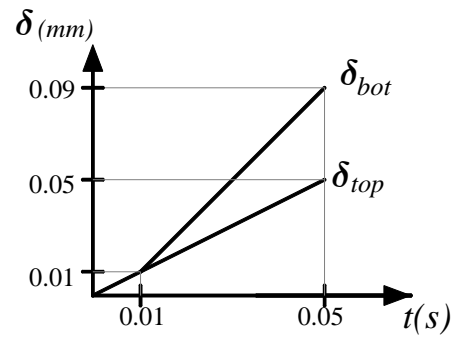

Figure B. 5 Bending of one element test: a) geometrical data, b)theoretical deformed shape, c)loading data.

This bending test will strongly challenge the constant displacement jump, since the theoretical solution of the problem involves both, a rigid body translation and rotation.

Figure B. 6 compares the results obtained with the standard $\dot{\hat{u}}, \llbracket \dot{u} \rrbracket$ and the proposed

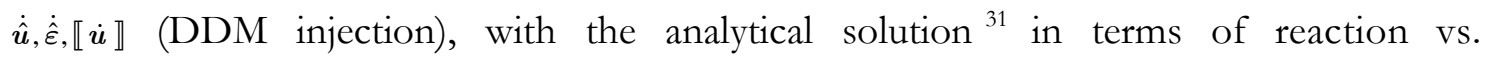
displacement curves. We remark that both formulations consider a constant displacement jump $\llbracket \dot{u} \rrbracket^{(e)}$. There, the stress-locking free character of the DDM injection can be observed. As expected, due the constant jump character of the displacement jump, the analytical solution is only linearly approximated by the DDM injection.

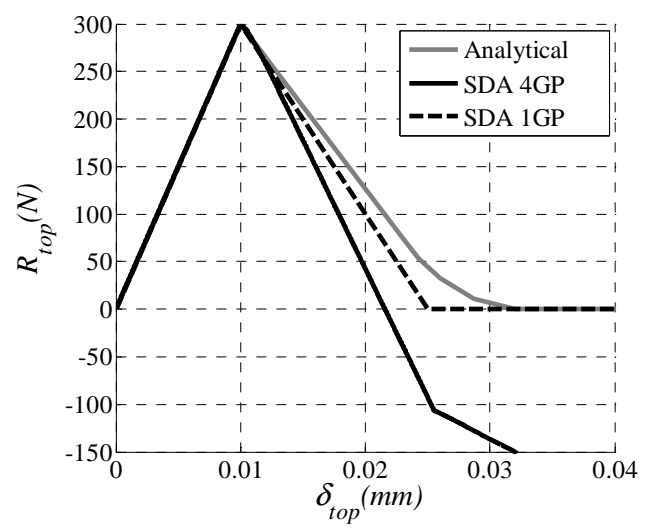

Figure B. 6 Reaction force vs. imposed displacement at the top comparing the analytical solution with solutions computed with the standard $\dot{\hat{u}}, \llbracket \dot{u} \rrbracket$ strong discontinuity formulation that is integrated with 4 Gauss points (SDA 4GP) and with the proposed $\dot{\hat{u}}, \dot{\hat{\varepsilon}}, \llbracket \dot{u} \rrbracket$ formulation that is integrated with two sampling points (SDA 1GP).

REMARK B. 1: In references (Manzoli and Shing 2006; Linder and Armero 2007) to avoid the stress locking problems associated to constant jump embedded formulations (for quads), a linear description of the jump along the discontinuity using 4 displacement degrees of freedom is proposed. As suggested by this analysis, a four degrees of freedom description of the kinematics can become excessive (in front of the three-degree-of-freedom kinematics in equation B.(50)) this explaining the additional stabilization treatment suggested in (Manzoli and Shing 2006; Linder and Armero 2007). Also, in 3D cases, six degrees of freedom should be used to describe the elemental displacement jump according with equation B.(49).

\footnotetext{
${ }^{31}$ The analytical solution is taken from (Linder and Armero 2007).
} 


\section{REFERENCES}

[1]Alfaiate, J., G. N. Wells and L. J. Sluys (2002). On the use of embedded discontinuity elements with crack path continuity for mode-I and mixed-mode fracture. Engineering Fracture Mechanics 69(6): 661-686.

[2]Alfaiate, J. (2003). New developments in the study of strong embedded discontinuities in finite elements. Advances in Fracture and Damage Mechanics 251-2: 109-114.

[3]Alfaiate, J., A. Simone and L. J. Sluys (2003). Non-homogeneous displacement jumps in strong embedded discontinuities. International Journal of Solids and Structures 40(21): 5799-5817.

[4]Armero, F. and K. Garikipati (1996). An analysis of strong discontinuities in multiplicative finite strain plasticity and their relation with the numerical simulation of strain localization in solids. Int.J. Solids and Structures 33(20-22): 2863-2885.

[5]Armero, F. (2012). Strong discontinuities in antiplane/torsional problems of computational failure mechanics. International Journal of Fracture 178(1-2): 3-32.

[6]Armero, F. and J. Kim (2012). Three-dimensional finite elements with embedded strong discontinuities to model material failure in the infinitesimal range. International Journal for Numerical Methods in Engineering: $n / a-n / a$.

[7]Arrea, M. and A. R. Ingraffea (1982). Mixed-mode Crack Propagation in Mortar and Concrete, Dept. Struct. Eng. Cornell Univ. New York.

[8]Bazant, Z. and J. Planas (1998). Fracture and size effect in concrete and other quasibrittle materials, CRC Press.

[9]Bazant, Z. P. (1983). Crack band theory for fracture of concrete. Materiaux et Constructions, Conference. 155-177.

[10]Belytschko, T. and W. E. Bachrach (1986). Efficient implementation of quadrilaterals with high coarse-mesh accuracy. Comput. Methods Appl. Mech. Eng. 54(3): 279-301.

[11]Belytschko, T., N. Moes, S. Usui and C. Parimi (2001). Arbitrary discontinuities in finite elements. International Journal for Numerical Methods in Engineering 50(4): 993-1013.

[12]Blanco, S., A. E. Huespe, J. Oliver and M. D. G. Pulido (2006). Modeling material failure in large concrete structures: Recent computational developments. Computational Modelling of Concrete Structures: 129-138.

[13]Bochev, P. and M. Gunzburger (2004). An Absolutely Stable Pressure-Poisson Stabilized Finite Element Method for the Stokes Equations. SIAM J. Numer. Anal. 42(3): 1189-1207.

[14]Brezzi, F. and M. Fortin (1991). Mixed and hybrid finite element methods, SpringerVerlag New York, Inc.

[15]Buscaglia, G. and V. Ruas (2013). Finite element methods for the Stokes system with interface

pressure discontinuitie. IMA Journal of Numerical Analysis.

[16]Cervera, M., M. Chiumenti and R. Codina (2010a). Mixed stabilized finite element methods in nonlinear solid mechanics. Part I: Formulation. Computer Methods in Applied Mechanics and Engineering 199(37-40): 2559-2570.

[17]Cervera, M., M. Chiumenti and R. Codina (2010b). Mixed stabilized finite element methods in nonlinear solid mechanics. Part II: Strain localization. Computer Methods in Applied Mechanics and Engineering 199(37-40): 2571-2589.

[18]Chen, J. F. (1975). Limit Analysis and Soil Plasticity, Elsevier.

[19]Contrafatto, L., M. Cuomo and F. Fazio (2012). An enriched finite element for crack opening and rebar slip in reinforced concrete members. International Journal of Fracture 178(1-2): 33-50. 
[20]de Borst, R. and H.-B. Mühlhaus (1992). Gradient-dependent plasticity: Formulation and algorithmic aspects. International Journal for Numerical Methods in Engineering 35(3): 521-539.

[21]Dias, I. F. (2012).Crack Path Field and Strain Injection Techniques in Numerical Modeling of Propagating Material Failure. Ph.D. Thesis, Ph.D. Thesis. Universitat Politècnica de Catalunya, (BarcelonaTech/UPC), Barcelona, Spain.

[22]Dias, I. F., J. Oliver and A. E. Huespe (2012). Strain Injection Techniques in Numerical Modeling of Propagating Material Failure. Monograph CIMNE No -134 . Barcelona. http://centrovnet.cimne.upc.edu/cvdata/cntr7/dtos/img/mdia/Downloads/M134.pdf , International Center for Numerical Methods in Engineering.

[23]Dohrmann, C. R. and P. B. Bochev (2004). A stabilized finite element method for the Stokes problem based on polynomial pressure projections. International Journal for Numerical Methods in Fluids 46(2): 183-201.

[24]Dufour, F., G. Pijaudier-Cabot, M. Choinska and A. Huerta (2008). Extraction of a crack opening from a continuous approach using regularized damage models. Computers \& Concrete 5(4): 375-388.

[25]Dugdale, D. (1960). Yielding of steel sheets containing slits. Journal for Mechanics of Phisics and Solids 8: 100-108.

[26]Francfort, G. A. and J. J. Marigo (1998). Revisiting brittle fracture as an energy minimization problem. Journal of the Mechanics and Physics of Solids 46(8): 1319-1342.

[27]Hillerborg, A., M. Modeer and P. E. Petersson (1976). Analysis of crack formation and crack growth in concrete by means of fracture mechanics and finite elements. Cement and concrete research $\mathbf{6}(6)$ : 773-782.

[28]Hughes, T. J. R. (1980). Generalization of selective integration procedures to anisotropic and nonlinear media. International Journal for Numerical Methods in Engineering 15(9): 1413-1418.

[29]Hughes, T. J. R. (1987). The Finite Element Method. Englewood Cliffs, N.J., PrenticeHall, Inc.

[30]Kobayashi, A. S., M. N. Hawkins, D. B. Barker and B. M. Liaw (1985). Fracture Process Zone of Concrete. Application of Fracture Mechanics to Cementitious Composites. S. P. Shah, Editor. Dordrecht, Marinus Nujhoff, Publications: 25-50.

[31]Kosloff, D. and G. A. Frazier (1978). Treatment of hourglass patterns in low order finite element codes. International Journal for Numerical and Analytical Methods in Geomechanics 2(1): 57-72.

[32]Linder, C. and F. Armero (2007). Finite elements with embedded strong discontinuities for the modeling of failure in solids. International Journal for Numerical Methods in Engineering 72(12): 1391-1433.

[33]Linero, D. L., J. Oliver, A. E. Huespe and M. D. G. Pulido (2006). Cracking modeling in reinforced concrete via the strong discontinuity approach. Computational Modelling of Concrete Structures: 173-182.

[34]Lordache, M.-M. and K. Willam (1998). Localized failure analysis in elastoplastic Cosserat continua. Comput. Methods Appl. Mech. Engrg. (151): 559-586.

[35]Malkus, D. S. and T. J. R. Hughes (1978). Mixed finite element methods - reduced and selective integration techniques: a unification of concepts. Comp. Meth. Appl. Mech. Eng. 15: 63-81.

[36]Manzoli, O. L. and P. B. Shing (2006). A general technique to embed non-uniform discontinuities into standard solid finite elements. Comput Struct 84(10-11): 742-757.

[37]Miehe, C., M. Hofacker and F. Welschinger (2010). A phase field model for rateindependent crack propagation: Robust algorithmic implementation based on operator splits. Computer Methods in Applied Mechanics and Engineering 199(45-48): 27652778 . 
[38]Miehe, C., F. Welschinger and M. Hofacker (2010). Thermodynamically consistent phase-field models of fracture: Variational principles and multi-field FE implementations. International Journal for Numerical Methods in Engineering 83(10): 1273-1311.

[39]Moës, N., N. Sukumar, B. Moran and T. Belytschko (2000). An extended finite element method (X-FEM) for two and three-dimensional crack modelling. ECCOMAS 2000, Conference.Barcelona, Spain, Vienna University of Technology Austria ISBN 39501554-0-6.

[40]Mosler, J. and G. Meschke (2003). 3D modelling of strong discontinuities in elastoplastic solids: fixed and rotating localization formulations. International Journal for Numerical Methods in Engineering 57(11): 1553-1576.

[41]Mosler, J. (2005). Numerical analyses of discontinuous material bifurcation: strong and weak discontinuities. Computer Methods in Applied Mechanics and Engineering 194(911): 979-1000.

[42]Muhlhaus, H. B. and I. Vardoulakis (1987). The thickness of shear bands in granular materials. Geotechnique 37(3): 271-283.

[43]Oliver, J. (1989). A Consistent Characteristic Length for Smeared Cracking Models. International Journal for Numerical Methods in Engineering 28(2): 461-474.

[44]Oliver, J., M. Cervera, S. Oller and J. Lubliner (1990). Isotropic damage models and smeared crack analysis of concrete. Proc. SCI-C Computer Aided Analysis and Design of Concrete Structures, Conference N. B. e. al. 945-957.

[45]Oliver, J. (1996a). Modelling strong discontinuities in solid mechanics via strain softening constitutive equations. Part 1. Fundamentals. International Journal for Numerical Methods in Engineering 39(21): 3575-3600.

[46]Oliver, J. (1996b). Modelling strong discontinuities in solid mechanics via strain softening constitutive equations. Part 2. Numerical simulation. International Journal for Numerical Methods in Engineering 39(21): 3601-3623.

[47]Oliver, J., M. Cervera and O. Manzoli (1999). Strong discontinuities and continuum plasticity models: the strong discontinuity approach. International Journal of Plasticity 15(3): 319-351.

[48]Oliver, J. (2000). On the discrete constitutive models induced by strong discontinuity kinematics and continuum constitutive equations. International Journal of Solids and Structures 37(48-50): 7207-7229.

[49]Oliver, J., A. E. Huespe, M. D. G. Pulido and E. Chaves (2002). From continuum mechanics to fracture mechanics: the strong discontinuity approach. Engineering Fracture Mechanics 69(2): 113-136.

[50]Oliver, J. and A. Huespe (2004a). Theoretical and computational issues in modelling material failure in strong discontinuity scenarios. Computer Methods in Applied Mechanics and Engineering 193(27-29): 2987-3014.

[51]Oliver, J. and A. E. Huespe (2004b). Continuum approach to material failure in strong discontinuity settings. Computer Methods in Applied Mechanics and Engineering 193(30-32): 3195-3220.

[52]Oliver, J., A. E. Huespe, E. Samaniego and E. W. V. Chaves (2004). Continuum approach to the numerical simulation of material failure in concrete. International Journal for Numerical and Analytical Methods in Geomechanics 28(7-8): 609-632.

[53] Oliver, J., A. Huespe, S. Blanco and D. Linero (2006a). Stability and robustness issues in numerical modeling of material failure with the strong discontinuity approach. Computer Methods in Applied Mechanics and Engineering 195(52): 7093-7114.

[54]Oliver, J., A. Huespe and P. Sanchez (2006b). A comparative study on finite elements for capturing strong discontinuities: E-FEM vs X-FEM. Computer Methods in Applied Mechanics and Engineering 195(37-40): 4732-4752. 
[55]Oliver, J., A. E. Huespe, J. C. Cante and G. Díaz (2010). On the numerical resolution of the discontinuous material bifurcation problem. International Journal for Numerical Methods in Engineering 83: 786-804.

[56]Oliver, J., A. E. Huespe and I. F. Dias (2012). Strain localization, strong discontinuities and material fracture: Matches and mismatches. Computer Methods in Applied Mechanics and Engineering 241-244: 323-336.

[57]Pijaudier Cabot, G. and Z. Bazant (1987). Nonlocal damage theory. Journal Engineering Mechanics ASCE 113: 1512-1533.

[58]Regueiro, R. A. and R. I. Borja (2001). Plane strain finite element analysis of presure sensitive plasticity with strong discontinuity. International Journal of Solids and Structures (38): 3647-3672.

[59]Rots, J. G. (1988).Computational Modeling of Concrete Fracture. Ph.D. Thesis, Delft University of Technology,

[60]Simo, J., J. Oliver and F. Armero (1993). An analysis of strong discontinuities induced by strain-softening in rate-independent inelastic solids. Computational Mechanics 12: 277-296.

[61]Simó, J. and J. Ju (1987). Strain and stress based continuum damage models. I. Formulation. International Journal of Solid and Structures 23: 821-840.

[62]Simó, J. and S. Rifai (1990). A class of mixed assumed strain methods and the method of incompatible modes. International Journal for Numerical Methods in Engineering 29: 1595-1638.

[63]Stakgold, I. (1998). Green's functions and boundary value problems. New York, Wiley.

[64]Stolarska, M., D. L. Chopp, N. Moës and T. Belytschko (2001). Modelling crack growth by level sets in the extended finite element method. Int.J.Num.Meth.Engng. 51: 943960. 